\title{
Measurement of the Top Quark Mass with In Situ Jet Energy Scale Calibration Using Hadronic $W$ Boson Decays AT CDF-II
}

by

Jean-François Arguin

A thesis submitted in conformity with the requirements for the degree of Doctor of Philosophy

Graduate Department of Physics

University of Toronto

Copyright (C) 2006 by Jean-François Arguin 


\begin{abstract}
Measurement of the Top Quark Mass with In Situ Jet Energy Scale Calibration Using Hadronic $W$ Boson Decays at CDF-II
\end{abstract}

\author{
Jean-François Arguin \\ Doctor of Philosophy \\ Graduate Department of Physics \\ University of Toronto \\ 2006
}

We report a measurement of the top quark mass with the upgraded Collider Detector at Fermilab (CDF-II). The top quarks are produced in pairs $(t \bar{t})$ in proton-antiproton collisions with a center-of-mass energy of $1.96 \mathrm{TeV}$. Each top quark decays to a $W$ boson and a bottom quark. We select candidate events in which one $W$ boson decays hadronically and the other decays to an electron or a muon and its associated neutrino. The data sample, which corresponds to an integrated luminosity of $318 \mathrm{pb}^{-1}$, contains $138 t \bar{t}$ candidates. A top quark mass is reconstructed for each event by placing energy and momentum constraints on the top quark pair decay products. We also employ the reconstructed mass of the hadronic $W$ boson decays $W \rightarrow j j$ to constrain in situ the largest systematic uncertainty of the top quark mass measurement, the jet energy scale. Monte Carlo templates of the reconstructed top quark and $W$ boson mass are produced as a function of the top quark mass and the jet energy scale. The distribution of reconstructed top quark and $W$ boson mass in the data are compared to the Monte Carlo templates using a likelihood fit to obtain $M_{t o p}=173.5_{-3.8}^{+3.9} \mathrm{GeV} / c^{2}$. This constitutes the most precise measurement of the top quark mass to date. This measurement can be used 
to constrain the mass of the Higgs boson, a central particle in the Standard Model of particle physics that has yet to be observed. We also demonstrate that this new technique reduces naturally the jet energy scale uncertainty as more data is accumulated and thus provides the capability to measure $M_{\text {top }}$ with an uncertainty of $2 \mathrm{GeV} / c^{2}$ or better by the end of the CDF-II experiment. 


\section{Acknowledgements}

First and foremost I am grateful to my Ph.D. supervisor, Pekka Sinervo. He's a deeply compassionate person, which I believe is the most important quality in a mentor. He has always been supportive, open-minded and available (despite important responsibilities). I also thank him for the example he is in every aspect of his professional life, in particular as a skillful physicist. He sets the bar high, but we better compare ourselves to the best! I feel privileged to have spent these years working with him.

Je remercie mes parents qui sont une grande source d'inspiration pour moi. J'admire spécialement en mon père son flair, sa profondeur et sa rigueur, et en ma mère sa compassion, sa détermination et sa générosité. Je leur suis aussi infiniment reconnaissant de leur support constant, surtout dans les moments les plus difficiles. Merci à mon frère, qui grâce son excellence académique, m'a inspiré à faire de même. Il est non seulement un frère, mais un grand ami et confident.

I had a great experience working with the CDF group in Toronto. I'd like to thank Pierre, William and Bob who helped me in many aspects of my student life. I enjoyed very much working with the post-docs and students of the group: Kostas, Simon, Oliver, Bernd, Dan, Shabnaz, Stan, Ian, Andrew, Reda, Rachid and Michael. I would like to thank Amita for her friendship and the good times in the first year of our Ph.D.'s in Toronto. I am also grateful to Bob Holdom and John Martin who have accepted to be part of my Ph.D. committee.

I would like to thank the people at the University of Chicago with whom I collaborated closely on the top quark mass project. I learned and matured a lot by working, sometimes on a day-to-day basis, with Young-Kee, Erik, Un-Ki, Mel and Jahred.

I am obliged to many people who participated like me to the Run II of the CDF experiment, specifically in the jet and top groups. In particular I would like to thank Beate, Lina, Doug, Anwar, Evelyn, Florencia, and Igor, who taught me each in a different manner how to become a better physicist. 
Mon expérience de vie à Toronto n'aurait jamais été la même sans l'amitié de Caroline, Anne, Ivan, Tagi, Jacques et plusieurs autres francophones de Toronto. J'aimerais aussi remercier mes amis de Montréal: Hugo, Thomas, Phanie, Dimitri, Ervig, Philippe, Frédéric et Marie, pour tous les bons moments passés ensemble, malgré la distance, à parler de d'autres choses que de physique! J'espère que l'avenir nous réserve de vivre plus près les uns des autres.

Finalement, j'aimerais remercier ma conjointe, Marie-Nadine, pour son amour, son support et sa patience. Elle a rendu ma vie heureuse à Toronto. Elle a toujours su me comprendre et me réconforter à travers toutes les difficultés et sacrifices associés avec des études graduées. Ce sera maintenant à mon tour de faire de même! J'espère faire aussi bien que toi. 


\section{Contents}

1 Introduction $\quad 1$

2 Theoretical Context $\quad 9$

2.1 Top Quark Phenomenology ... . . . . . . . . . . . . 10

2.1.1 General Picture of Top Quark . . . . . . . . . . . . . 10

2.1.2 Top Quark Production . . . . . . . . . . . . . 11

2.1 .3 Top Quark Decay . . . . . . . . . . . . . . . . 12

2.2 Motivation for a Precise Top Quark Mass Measurement . . . . . . . . . 15

2.2 .1 Precision Electroweak fits . . . . . . . . . . 16

2.2.2 The Top Quark Beyond the Standard Model . . . . . . . . . . 18

3 Experimental Apparatus $\quad 24$

3.1 The Fermilab's Accelerator Chain . . . . . . . . . . . . . . . 24

3.1 .1 The Proton Source . . . . . . . . . . . . 25

3.1 .2 The Main Injector . . . . . . . . . . . 26

3.1.3 The Antiproton Source . . . . . . . . . . . . 26

3.1.4 The Tevatron .................... 27

3.2 The CDF-II Detector . . . . . . . . . . . . . . . . 27

3.2.1 Silicon Detectors . . . . . . . . . . . . 30

3.2 .2 Central Outer Tracker ................... 31

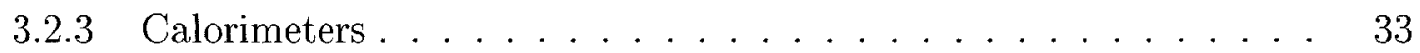

vii 
3.2 .4 Muon Detectors . . . . . . . . . . . . . . . . . . . . 35

3.2 .5 Cherenkov Luminosity Counters . . . . . . . . . . . . . . . 36

3.2.6 Triggers and Data Acquisition System _. . . . . . . . . 37

3.3 Monte Carlo Generation and Detector Simulation . . . . . . . . . . . . 39

4 Selecting the $t \bar{t}$ Events $\quad 42$

4.1 Trigger Requirements . . . . . . . . . . . . . . . . . . . . . . . 43

4.1 .1 Central Electron Trigger . . . . . . . . . . . . . . . . . 43

4.1 .2 Central Muon Trigger f . . . . . . . . . . . . . . . . . 44

4.2 Offline Event Selection and Reconstruction . . . . . . . . . . . . . . 44

4.2 .1 Lepton Track Selection . . . . . . . . . . . . . . . . . 45

4.2 .2 Electron Selection . . . . . . . . . . . . . . . . . . 46

4.2 .3 Muon Selection . . . . . . . . . . . . . . . . . . . 47

4.2 .4 Jet Reconstruction . . . . . . . . . . . . . . . . . . . . . 48

4.2 .5 Missing Transverse Energy . . . . . . . . . . . . . . . . . 55

$4.2 .6 \quad$ Final Event Selection . . . . . . . . . . . . . . . . . . 55

4.3 Division of the Lepton+Jets Sample . . . . . . . . . . . . . . . 56

4.4 Background Contamination $\ldots \ldots \ldots \ldots \ldots$

5 Top Quark Mass Reconstruction $\quad 62$

5.1 The $\chi^{2}$ Kinematic Fit . . . . . . . . . . . . . . . . . . . . 62

5.2 Description of the Input to the $\chi^{2}$ Fit $\ldots \ldots \ldots$. . . . . . . 64

5.2 .1 Lepton . . . . . . . . . . . . . . . . . . . . . . 64

5.2 .2 Jets $\ldots \ldots \ldots \ldots \ldots \ldots \ldots$

5.2 .3 Unclustered Energy . . . . . . . . . . . . . . . . 67

5.3 The $\chi_{m i n}^{2}$ Cut . . . . . . . . . . . . . . . . 69

5.4 Definition of the Jet Energy Scale Uncertainty . . . . . . . . . . . 70

5.5 Signal Templates . . . . . . . . . . . . . . . . 75

viii 
5.6 Background Templates . . . . . . . . . . . . . 78

6 W Boson Mass Reconstruction $\quad 82$

6.1 Reconstruction Method for $m_{j j} \ldots \ldots \ldots \ldots$. . . . . . . . . . 82

6.2 Signal Templates . . . . . . . . . . . . . . . . . 85

6.3 Background Templates . . . . . . . . . . . . . . . 86

$\begin{array}{lll}7 \text { Likelihood Fits } & 91\end{array}$

7.1 General Strategy . . . . . . . . . . . . . . . . . . 91

7.2 Jet Energy Scale Cross-Check . . . . . . . . . . . . . . . . 92

7.2 .1 Description of the Likelihood Fit . . . . . . . . . . 92

7.2 .2 Pseudo-Experiments Procedure . . . . . . . . . . . 94

7.2.3 Assessment of the Likelihood Performance . . . . . . . . . . . 95

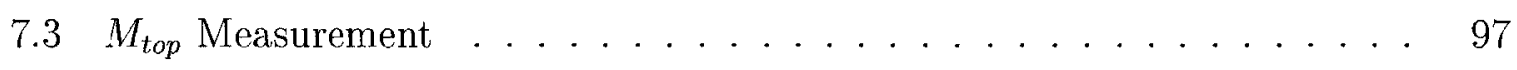

7.3 .1 Description of the Likelihood Fit . . . . . . . . . . 98

7.3.2 Pseudo-Experiments Procedure . . . . . . . . . . . . 101

7.3.3 Assessment of the Likelihood Performance . . . . . . . . . . . . 102

7.3.4 Further Checks . . . . . . . . . . . . . . 104

8 Systematic Uncertainties $\quad 110$

$8.1 \quad B$-Jets Energy Scale . . . . . . . . . . . . . . . 110

8.2 Method Uncertainty ......................... 113

8.3 Hard QCD Radiation . . . . . . . . . . . . . . . 115

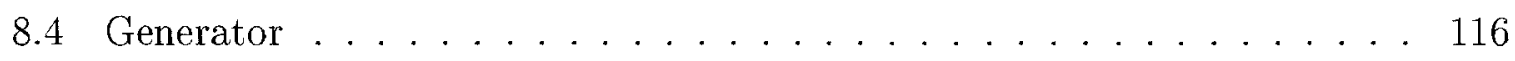

8.5 Parton Distribution Functions . . . . . . . . . . . . . 117

8.6 Background Shape . . . . . . . . . . . . . . . . . . . 119

8.7 Background Jet Energy Scale . . . . . . . . . . . . . . . 121

8.8 Monte Carlo Statistics . . . . . . . . . . . . . . . . 123

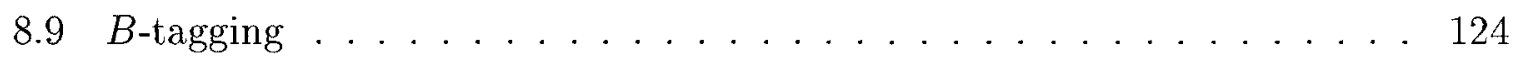


$8.10 M_{\text {top }}$ Uncertainty for the JES Cross-Check . . . . . . . . . . 125

8.11 Summary . . . . . . . . . . . . . . . . . 125

9 The Top Quark Mass Measurement 127

9.1 Application of the JES Cross-Check to Data . . . . . . . . . . . 128

9.1.1 Final Result for the JES Cross-Check . . . . . . . . . . . . . . . . 130

9.2 Application of the $M_{t o p}$ Measurement to Data . . . . . . . . . . 131

9.2.1 Final Result for the $M_{t o p}$ Measurement . . . . . . . . . . . . 138

10 Conclusions $\quad 142$

$\begin{array}{lr}\text { Contributions } & 149\end{array}$

$\begin{array}{ll}\text { Glossary } & 150\end{array}$

$\begin{array}{lr}\text { Bibliography } & 156\end{array}$ 


\section{List of Tables}

1.1 The six known quarks in nature and their properties. . . . . . . . . 2

1.2 The six known leptons in nature and their properties. . . . . . . . . . 3

2.1 Branching ratios of the $t \bar{t}$ experimental signatures. . . . . . . . . . . 14

4.1 Jet $E_{r}$ cut, $b$-tagging requirement, expected signal to background ratio and the number of events observed in data for the four event categories. . 59

4.2 Background event estimates for the $W$ boson mass sample. . . . . . . . . 60

4.3 Background event estimates for the top quark mass sample. . . . . . . 61

5.1 Signal efficiency for cut $\chi_{\min }^{2}<9$ for $M_{t o p}=178 \mathrm{GeV} / c^{2} \ldots \ldots . \ldots 9$

5.2 The $\chi^{2} /$ ndof for the $m_{t}^{r e c o}$ signal templates. . . . . . . . . . . 78

6.1 The $\chi^{2} / n d o f$ for the $m_{j j}$ signal templates. . . . . . . . . . . 89

7.1 Expected number of signal events in the $m_{t}^{\text {reco }}$ and $m_{j j}$ samples. . . . . 95

7.2 The $\chi_{\min }^{2}$ cut efficiencies for signal and background extracted at nominal JES and $M_{\text {top }}=178 \mathrm{GeV} / c^{2}$ for signal. . . . . . . . . 100

8.1 Uncertainty from the specific characteristics of $b$-jets. . . . . . . . 113

8.2 Shift in $M_{\text {top }}$ and JES due to variations of the ISR, FSR and MC generator. 116

8.3 Systematic shift in $M_{t o p}$ and JES due to different PDFs. . . . . . . 118

8.4 Systematic shifts due to variations of the background shapes. . . . . . . . 119

8.5 Systematic shifts due to variations of the JES for the background shapes. 123 
8.6 Summary of the uncorrelated systematic uncertainties . . . . . . . . . . 126

9.1 The fitted number of signal and background events in each subsample for the JES cross-check. . . . . . . . . . . . . . . . . . . 132

9.2 The fitted number of signal and background events in each subsample for the JES cross-check where the background is left unconstrained for all subsamples. . . . . . . . . . . . . . . . 132

9.3 Results of the JES cross-check in each subsample. . . . . . . . . . . . 133

9.4 The fitted number of signal and background events in each subsample for the $M_{\text {top }}$ measurement. . . . . . . . . . . . . . . 139

9.5 The fitted number of signal and background events in each subsample for the $M_{\text {top }}$ measurement where the background is left unconstrained for all

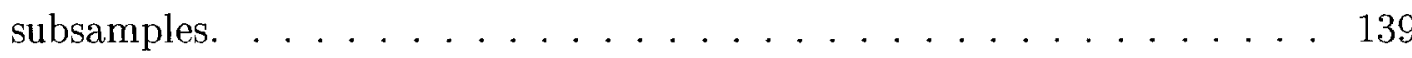

9.6 Results of the $M_{\text {top }}$ measurement in each subsample. . . . . . . . . . . . . 140 


\section{List of Figures}

1.1 Summary of top quark mass measurements as of April 2005. . . . . . . . 6

2.1 Tree level Feynman diagrams for the strong interactions (a) $q \bar{q} \rightarrow t \bar{t}$ and

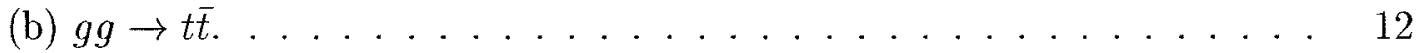

2.2 Cross-section of $t \bar{t}$ pair production as a function of $\sqrt{s}$ for the theory prediction and CDF measurements. . . . . . . . . . 13

2.3 Diagram of a lepton+jets $t \bar{t}$ event. . . . . . . . . . . . . 15

2.4 The propagators of the $W$ and $Z$ bosons where a loop involving top quarks

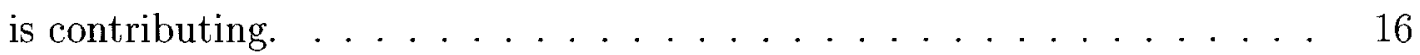

2.5 Constraint on the Higgs boson mass as a function of the top quark and $W$ boson measured masses as of spring of 2005 .

2.6 Loop contributions to the Higgs boson propagator from (a) fermionic and (b) scalar particles. . . . . . . . . . . . . . . .

2.7 Experimental constraints on Supersymmetry from measurements of $M_{W}$ and $M_{t o p}$ (using Run I world average). . . . . . . . . . .

3.1 A diagram of the Fermilab's accelerator chain. . . . . . . . . . . . 25

3.2 Initial instantaneous luminosity at CDF as a function of store number and date of data taking (June 2001 to June 2005). . . . . . . . . . . 28

3.3 Elevation view of one-half of the CDF-II detector. . . . . . . . . . 29

3.4 End view of the CDF-II silicon system. . . . . . . . . 30 
3.5 Side view of one half of the CDF-II silicon system. . . . . . . . . . . 32

3.6 Cross section of the upper part of the plug calorimeter. . . . . . . . . . . 34

3.7 Number of absorption lengths as a function of the pseudorapidity for the CMU, CMP and CMX detectors. . . . . . . . . . . . . 37

3.8 Diagram of the data flow in the CDF trigger and data acquisition system. 41

4.1 Distributions of $L_{s h r}, E / P, E_{h a d} / E_{e m}$ and isolation energy for electrons in $Z \rightarrow$ ee data events.

4.2 Distributions of the distance in the axial direction between the extrapolated track and muon stub positions in the CMU and CMX detectors in $Z \rightarrow \mu \mu$ data events.

4.3 Ratio of the $p_{T}$ of the probe and trigger jets as a function of $\eta_{\text {det }}$ of the probe jet in dijet balancing for data and Monte Carlo events. . . . . . . 51

4.4 Absolute energy scale correction as a function of the jet $p_{T} \ldots \ldots$. . . . 53

4.5 SECVTX $b$-tagging efficiency as a function of the uncorrected jet $p_{T}$ in the region $\left|\eta_{\text {det }}\right|<1 . \ldots \ldots \ldots \ldots \ldots \ldots$

4.6 Reconstructed top quark mass $\left(m_{t}^{r e c o}\right)$ for $t \bar{t}$ events with $M_{t o p}=178 \mathrm{GeV} / \mathrm{c}^{2} .58$

5.1 Correction factors of the top-specific corrections. . . . . . . . . 66

5.2 Jet $p_{T}$ resolution of the top-specific corrections. . . . . . . . . . . 67

5.3 Sketch of the production and detection of a jet. . . . . . . 71

5.4 Relative jet energy systematic uncertainty as a function of $\left|\eta_{\text {det }}(j e t)\right| \cdot$. . 72

5.5 Absolute jet energy scale uncertainty. . . . . . . . . . . . . 73

5.6 Systematic uncertainty from out-of-cone energy for cone $\Delta R=0.4$. . . 74

5.7 Total jet energy scale uncertainty $\left(+1 \sigma_{c}\right)$ as a function of the corrected jet

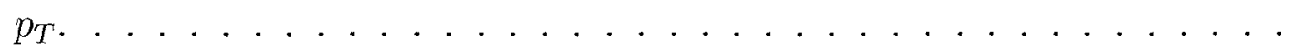

5.8 Reconstructed top quark mass distributions for various top quark mass $\left(\mathrm{JES}=0 \sigma_{c}\right)$ for the $1-\operatorname{tag}(\mathrm{T})$ subsample $\ldots \ldots \ldots 76$ 
5.9 Reconstructed top quark mass distributions for various value of JES ( $M_{\text {top }}$ $\left.=180 \mathrm{GeV} / c^{2}\right)$ for the $1-\operatorname{tag}(\mathrm{T})$ subsample. . . . . . . . . . 77

5.10 Combined background $m_{t}^{r e c o}$ templates. . . . . . . . . . . . . 81

6.1 Reconstructed hadronic $W$ boson mass from HERWIG $t \bar{t}$ events with nominal JES and $M_{\text {top }}=178 \mathrm{GeV} / \mathrm{c}^{2} \ldots \ldots \ldots \ldots$. . . . . . . 84

6.2 Reconstructed $W \rightarrow j j$ mass distributions for various value of JES $\left(M_{t o p}\right.$ $=180 \mathrm{GeV} / c^{2}$ ) for the 2 -tag subsample.

6.3 Reconstructed $W \rightarrow j j$ mass distributions for various top quark masses (nominal JES) for the 2 -tag subsample. . . . . . . . . . . . 87

6.4 Probability density function of $m_{j j}$ versus $m_{j j}$ and JES for the 2-tag subsample $\left(M_{t o p}=180 \mathrm{GeV} / c^{2}\right)$.

6.5 Probability density function of $m_{j j}$ versus $m_{j j}$ and $M_{t o p}$ for the 2-tag subsample (nominal JES). . . . . . . . . . . . . . . . . . 89

6.6 Combined background $m_{j j}$ templates. . . . . . . . . . . . . 90

7.1 Mean and width of the JES pull distributions as a function of the input JES for the JES cross-check. . . . . . . . . . . . . . . . . 97

7.2 JES statistical uncertainty as a function of input JES for the JES cross-check. 98

7.3 Mean of the $M_{t o p}$ pull distributions as a function of the input $M_{t o p}$ and the input JES for the $M_{t o p}$ measurement. . . . . . . . . . . . . . 103

7.4 Mean of the JES pull distributions as a function of the input $M_{t o p}$ and the input JES for the $M_{t o p}$ measurement.

7.5 Width of the $M_{t o p}$ pull distributions as a function of the input $M_{t o p}$ and the input JES for the $M_{\text {top }}$ measurement. . . . . . . . . . . . 105

7.6 Width of the JES pull distributions as a function of the input $M_{\text {top }}$ and the input JES for the $M_{\text {top }}$ measurement. . . . . . . . . . 106 
7.7 Median of the expected $M_{t o p}$ uncertainties as a function of the input $M_{t o p}$ and the input JES for the $M_{\text {top }}$ measurement for $\int \mathcal{L} d t=318 \mathrm{pb}^{-1} \ldots \ldots 107$

7.8 Median of the expected JES uncertainties as a function of the input $M_{\text {top }}$ for the $M_{\text {top }}$ measurement for $\int \mathcal{L} d t=318 \mathrm{pb}^{-1} \ldots \ldots \ldots$. . . . . . . 108

7.9 The three $m_{j j}$ combinations in the $1-\operatorname{tag}(\mathrm{T})$ subsample versus $m_{t}^{\text {reco }}$ for a signal sample with $M_{\text {top }}=178 \mathrm{GeV} / c^{2} \ldots \ldots \ldots \ldots 8$

7.10 Mean and width of the $M_{t o p}$ pull distribution as a function of the input $M_{\text {top }}$ for the $M_{\text {top }}$ measurement where the correlations between the reconstructed masses in each event are simulated. . . . . . . . . . . . . . . 109

8.1 An example of a $p_{T}$-dependent variation of the JES discrepancy between data and MC . . . . . . . . . . . . . . . 114

8.2 A tree level diagram of a $t \bar{t}$ event where a gluon is radiated from the incoming top quark (ISR) and $b$ quark propagators (FSR) . . . . . . . 115

8.3 Median of fitted $M_{\text {top }}$ from pseudo-experiments for various PDF sets. . . 118

8.4 Background $m_{t}^{r e c o}$ shape for the fake electron data and the $W+$ jets MC. . 121

8.5 Background $m_{j j}$ shape for the fake electron data and the $W+$ jets MC. $\quad 122$

8.6 Median of fitted $M_{\text {top }}$ distribution of pseudo-experiments for 100 statistically fluctuated signal templates. . . . . . . . . . . . . . . 124

9.1 Data $m_{j j}$ distributions with the signal and background template shapes corresponding to the best fit of the JES cross-check overlaid on the histograms. The value of $M_{t o p}$ has been constrained to $178 \mathrm{GeV} / c^{2} \ldots \ldots 129$

9.2 Negative log-likelihood function as a function of JES for the JES cross-check. 130

9.3 Negative and positive uncertainties based on the number of observed events for the JES cross-check. . . . . . . . . . . . . . . . . . 131

9.4 Negative log-likelihood as a function of JES for the JES cross-check for the various subsamples. . . . . . . . . . . . . . . . . . . 134 
9.5 Data $m_{t}^{r e c o}$ distributions with the template mass distributions for the best fit of the $M_{t o p}$ measurement overlaid on the histograms. Both $M_{t o p}$ and JES are free parameters in the likelihood fit. . . . . . . . . . . 135

9.6 Data $m_{j j}$ distributions with the template mass distributions for the best fit of the $M_{t o p}$ measurement overlaid on the histograms. Both $M_{t o p}$ and JES are free parameters in the likelihood fit. . . . . . . . . . . . 136

9.7 Negative log-likelihood as a function of $M_{t o p}$ and JES for the $M_{t o p}$ measurement. . . . . . . . . . . . . . . . . 137

9.8 Negative and positive $M_{\text {top }}$ and JES uncertainties based on the number of observed events for the $M_{\text {top }}$ measurement. . . . . . . . . . . . . . . 138

9.9 Negative log-likelihood function as a function of $M_{t o p}$ and JES for the various subsamples for the $M_{\text {top }}$ measurement. . . . . . . . . . . . . 141

10.1 Contours of $68 \%$ confidence level in the $M_{t o p}-M_{W}$ plane and the updated constraint on the Higgs boson mass as a function of $M_{t o p}$ and $M_{W}$. . . 145

10.2 Updated experimental constraints on Supersymmetry from measurements of $M_{W}$ and $M_{t o p}$ (using Run I world average). . . . . . . . . 146

10.3 JES uncertainty from $W \rightarrow j j$ measurement only as a function of integrated luminosity. . . . . . . . . . . . . . . . . . 147

10.4 Total $M_{t o p}$ uncertainty as a function of integrated luminosity. . . . . . . 148 


\section{Chapter 1}

\section{Introduction}

Since the origin of time, the human race has attempted to comprehend the apparent complexity of nature. One popular approach consists in searching for common characteristics or patterns in observations that can lead to a more fundamental understanding of matter, light and the forces of nature. This reductionist approach has been very successful in the last two centuries. From the work of Dalton, Mendeleev and others we understood in the last century that all matter was constituted of only about 100 fundamental elements that were named atoms to honor ancient Greek philosophers Leucippus and Democritus, who first hypothesized the indivisibility of matter. At the beginning of the last century, experiments lead by Thomson [1], Rutherford [2] and others demonstrated that atoms were themselves made of smaller constituents: the electron, a particle with a negative electric charge orbiting around a small nucleus that is made of nucleons, i.e. protons (positive electric charge) and neutrons (neutral electric charge).

The existence of two forces acting upon particles of matter was obvious at least since antiquity: electricity (later known as electromagnetism, see below) and gravity. However, it was soon apparent that additional forces existed, both to explain radioactive phenomena first observed by Becquerel [3] (the weak force) and to counteract the electrostatic repulsion of protons inside nuclei (the strong force). The existence of another stable but 


\begin{tabular}{ccc}
\hline \hline Name & Electric charge $(\mathrm{e})$ & Mass $\left(\mathrm{MeV} / c^{2}\right)$ \\
\hline up $u$ & $+2 / 3$ & $1.5-4$ \\
down $d$ & $-1 / 3$ & $4-8$ \\
strange $s$ & $-2 / 3$ & $80-130$ \\
charm $c$ & $+2 / 3$ & $1.15-1.35 \times 10^{3}$ \\
bottom $b$ & $-1 / 3$ & $4.1-4.9 \times 10^{3}$ \\
$\operatorname{top} t$ & $+2 / 3$ & $1.780 \pm 0.043 \times 10^{5}$ \\
\hline \hline
\end{tabular}

Table 1.1: The six known quarks in nature and their properties. We show the allowed range in the theoretical determination of the mass of the first five quarks [5]. The uncertainty on the top quark mass is experimental (68\% confidence level) [6].

weakly interacting particle, the neutrino, was later hypothesized to be emitted in the weak decays of neutrons ( $\beta$ decay) in association with electrons.

Just as the existence of three particles (two nucleons and the electron) explained the existence of about 100 type of atoms, sub-components of the nucleons, named quarks, have been hypothesized to explain the plethora of observed particles in high-energy experiments in the second half of the 20th century. The existence of quarks was later confirmed by the study of high energetic electrons scattering off nuclei. Under the quark model developed by Gell-Mann and others [4], the proton is made of two up quarks and one down quark, while the neutron is made of two down quarks and one up quark. Fractional electric charge of $+2 / 3$ and $-1 / 3$ are assigned to the up and down quarks, respectively, to explain the observed charge of the nucleons. Six quarks have been observed to date, the last one being the top quark, which is the focus of this dissertation. There are also six known leptons, i.e. particles that do not interact via the strong force, like the electron and the neutrinos. The leptons and quarks are currently regarded as the fundamental building blocks of matter. The names, electric charges and masses of quarks and leptons are given in Tables 1.1 and 1.2 , respectively. 


\begin{tabular}{ccc}
\hline \hline Name & Electric charge (e) & Mass $\left(\mathrm{MeV} / c^{2}\right)$ \\
\hline Electron $e^{-}$ & -1 & $0.51099892 \pm 0.00000004$ \\
Electron neutrino $\nu_{e}$ & 0 & $<3 \times 10^{-6}$ \\
Muon $\mu^{-}$ & -1 & $105.658369 \pm 0.000009$ \\
Muon neutrino $\nu_{\mu}$ & 0 & $<0.19$ \\
Tau $\tau^{-}$ & -1 & $<18.2$ \\
Tau neutrino $\nu_{\tau}$ & 0 & $<9.29$ \\
\hline \hline
\end{tabular}

Table 1.2: The six known leptons in nature and their properties [5]. The uncertainties on the charged lepton masses represent the $68 \%$ confidence level. The neutrino mass limits are $95 \%, 90 \%$ and $95 \%$ confidence level limits for $\nu_{e}, \nu_{\mu}$ and $\nu_{\tau}$, respectively. Although the absolute mass of neutrinos have not been measured yet, neutrino oscillations experiments recently determined that the mass difference between neutrino types, and thus the masses themselves, are non-zero.

The reductionist approach also played a central role in the theoretical development of elementary particle physics. One early example is the unification of the electrical and magnetic forces under electromagnetism by Maxwell. The two major physics theories developed at the beginning of the 20th century - quantum mechanics that describes particles behavior at the microscopic level, and special relativity that describes particles traveling near the speed of light - were later unified with the work of Dirac, Feynman and others into quantum field theory, which is the basis of modern day theories of particle physics. The role of symmetry in physics and in particular its link to conservation laws as stated by Noether's theorem has become increasingly important in the development of modern physics. For instance, it has been realized that requiring the invariance of the Lagrangians describing the dynamics of a system under $\mathrm{U}(1), \mathrm{SU}(2)$ and $\mathrm{SU}(3)$ group (local) transformations requires the existence and reproduces the properties of the force carriers of the electromagnetic (photon), weak ( $W^{ \pm}$and $Z$ bosons) and strong (gluon) 
forces, respectively. Theories with such features are denoted as "gauge" theories. The theory that describes the behavior of quarks and leptons under the electromagnetic, weak and strong forces is often denoted as the "Standard Model" (SM) of particle physics. Its predictions are in very good agreement with experimental observations. One great success of the SM is the discovery of the predicted $W$ and $Z$ boson at CERN in 1983 [7].

The current experimental data establishes the existence of the six quarks, six leptons, and the force carriers of the three forces predicted by the SM. These data also require the theory to give each fermion a mass, and to predict non-zero masses for the $W$ and $Z$ bosons. This is achieved through a mechanism known as spontaneous symmetry breaking that is mediated by the Higgs boson. The Higgs boson is the last particle required for the internal consistency of the SM that has yet to be observed. It is central to the theory and the quest for the Higgs has been compared to the "Holy Grail" of contemporary particle physics. For instance, the emphasis of the next generation of high-energy colliders, the Large Hadron Collider (LHC) aiming to start operating in 2007, is on the discovery and study of the Higgs boson. Interestingly, a direct observation is not the only way to gather information on this important particle. Precise measurements of Standard Model observables and the predictive power of the theory can be used to constrain the Higgs model. One such observable is the top quark mass $\left(M_{t o p}\right)$ that can provide significant constraints on the Higgs boson mass. This role for the top quark in the indirect determination of the Higgs boson mass and more generally as a self-consistency check of the Standard Model strongly motivates a precise measurement of the top quark mass. Indeed, it is currently considered one of the key measurements in high-energy physics.

This dissertation presents the most precise measurement of the top quark mass to date. The only apparatus in operation with sufficient center-of-mass energy to produce significant numbers of top quarks is the Tevatron, a proton-antiproton collider located at the Fermi National Accelerator Laboratory in Illinois. The top quarks are produced primarily as top-antitop pairs $(t \bar{t})$. As will be described in more detail in Chapter 2, the 
lifetime of the top quark is so short that it decays before forming hadrons (i.e. bound state of quarks). Therefore, the mass of the top quark can be inferred by direct reconstruction of the momentum and energy of its decay products. Each top quark decays to a $W$ boson and a bottom quark $(b)$, and the $W$ bosons in turn decay to two quarks (hadronic decay) or a charged lepton and a neutrino (leptonic decay). The measurement presented here uses events where one $W$ boson decays hadronically and the other leptonically (referred to as the lepton+jets channel). Other channels, such as the "dilepton" channel where both $W$ bosons decay leptonically and the "all-hadronic" channel where both $W$ bosons decay hadronically, can be used for top quark studies but the lepton+jets channel is ideal for the top quark mass measurement. Indeed, it suffers from less background than the all-hadronic channel and the events can be fully reconstructed, which is not the case for the dilepton channel that is complicated by the presence of two neutrinos that escape the detector without interacting. The $t \bar{t}$ events are detected by the CDF [8] and DØ [9] detectors located at collision points on the Tevatron Ring. The CDF and DØ experiments have been in operation in runs of data-taking from 1987 to 1995. The current run that started in 2001 is referred to as Run II. The measurement presented here uses data collected by Run II of the CDF detector between March 2002 and August 2004 for a total integrated luminosity of $318 \mathrm{pb}^{-1}$.

Due to the complicated topology of the $t \bar{t}$ events, there is no single "best" way to measure the top quark mass. One strategy is to identify a variable that is sensitive to the top quark mass and perform the measurement based on the distribution of this variable observed in the data. A simple example of such a variable is the sum of all the energy deposited in the CDF detector by the decay products of the top quark (often denoted as $H_{T}$ ). This variable is dependent on the top quark mass as larger values of $M_{\text {top }}$ result in more energy deposited in the CDF detector on average. Monte Carlo simulations of $t \bar{t}$ events interacting in the CDF detector can be used to produce $H_{T}$ distributions that we can expect to observe as a function of the top quark mass. These 


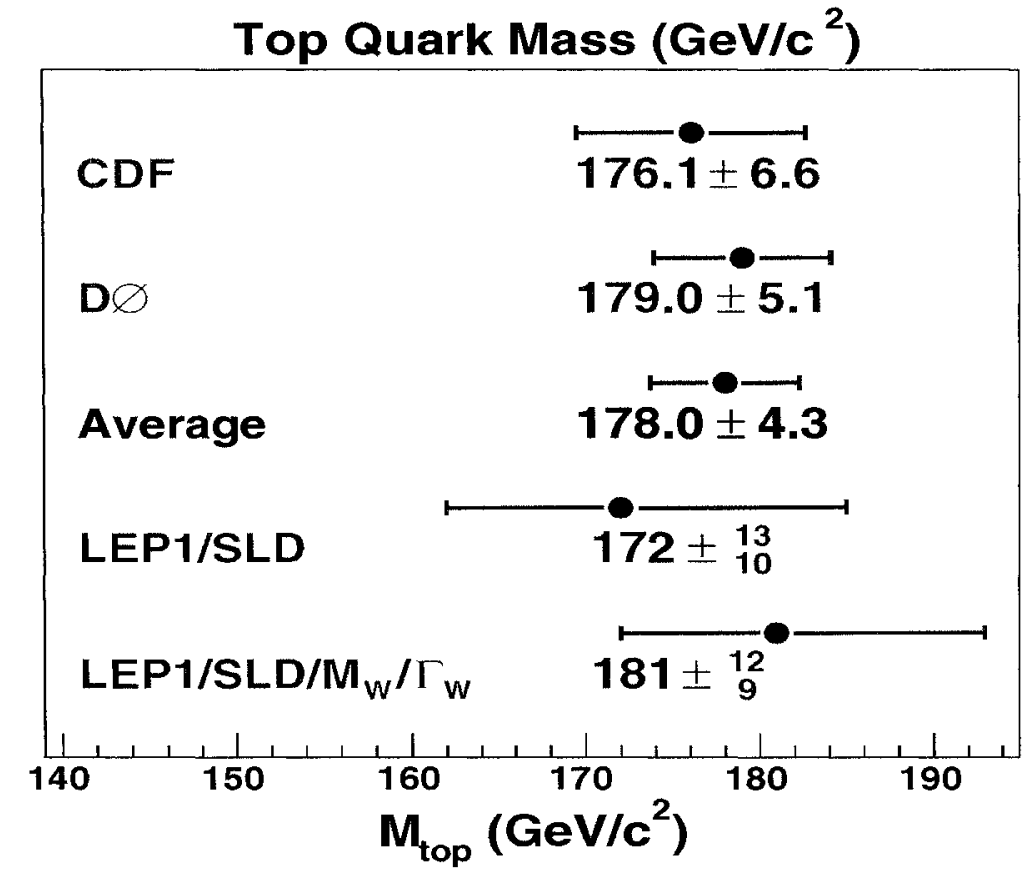

Figure 1.1: Summary of top quark mass measurements as of April 2005 [15].

distributions are referred to as "templates." The measured top quark mass is the one that corresponds to the template that matches best the $H_{T}$ distribution observed in the data. This technique is generally referred to as the "template method" and has been used widely in the past [10] where the most commonly employed variable is the reconstructed top quark mass defined in Chapter 5 of this thesis. A different and more recent class of methods calculates an event-by-event likelihood as a function of $M_{\text {top }}$ based on the full Standard Model calculation of $t \bar{t}$ production and decay. This method is referred to as the "matrix-element" method and has provided precise measurements, including the best measurement performed during Run I [11]. Other measurements have been performed in the dilepton $[12,13]$ and the all-hadronic [14] channels but with larger uncertainties. The knowledge of the top quark mass as of April 2005 is summarized in Fig. 1.1. Indirect constraints are extracted from a Standard Model fit of data gathered at the LEP and SLD experiments. The world average from direct measurements is $M_{t o p}=178.0 \pm 4.3 \mathrm{GeV} / c^{2}$ $[6]$. 
The analysis presented in this document uses a novel technique based on the template method that aims to improve the dominant systematic uncertainty of traditional top quark mass measurements: the jet energy scale (JES) uncertainty. Jets can be described as the flow of particles resulting from the hadronization of high energy quarks or gluons. There are large uncertainties associated with the energy measurements of these complicated objects (as described in detail in Chapter 5). The impact on the top quark mass measurement is amplified by the fact that a large number of jets (at least four) are expected in lepton+jets final state. For instance, $2.6 \mathrm{GeV} / c^{2}$ of the world average uncertainty of $4.3 \mathrm{GeV} / c^{2}$ comes from the jet energy scale uncertainty. More importantly the statistical uncertainty (currently $2.7 \mathrm{GeV} / c^{2}$ of the world average) will naturally decrease as more data are accumulated by the CDF and DØ experiments, making the jet energy scale a greater problem for future precision measurements. The basic idea behind the new technique is to calibrate in situ the jet energy measurements by monitoring the mass of the $W$ boson decaying hadronically inside the $t \bar{t}$ events. We will demonstrate that this technique reduces significantly this dominant systematic uncertainty.

The outline of this thesis is as follows: we discuss in Chapter 2 the general theory of top quark physics and motivate the measurement of $M_{\text {top }}$. The experimental apparatus employed to perform this measurement, i.e. the Tevatron collider and the CDF detector, are described in Chapter 3. The selection of the $t \bar{t}$ events and the background contamination of the resulting dataset are presented in Chapter 4 . The reconstruction of the top quark mass $\left(m_{t}^{r e c o}\right)$ event-by-event and the associated templates are the subject of Chapter 5 . The in situ calibration of the jet energy scale necessitates the reconstruction of hadronic $W$ boson mass $\left(m_{j j}\right)$ templates that is discussed in Chapter 6 . The top quark mass is extracted by comparing the top quark and $W$ boson mass distributions reconstructed in data with the templates obtained from simulated events with various hypotheses of $M_{\text {top }}$ and JES. This measurement is performed with a likelihood fit procedure presented in Chapter 7 . The systematic uncertainties of the measurement are 
estimated in Chapter 8 and the results of the application of the likelihood fit to data are discussed in Chapter 9. Finally, further discussions about the impact of the results and the future of this technique are presented in Chapter 10. A glossary for non-particle physicists is included at the end of this dissertation. 


\section{Chapter 2}

\section{Theoretical Context}

The first observations of the top quark were reported only ten years ago by the CDF and D $\varnothing$ experiments [16]. The discovery of the top quark was not a surprise. Indeed, the existence of an isospin partner for the $b$-quark is strongly motivated by arguments of theoretical consistency of the Standard Model, absence of flavor neutral changing current in $B$ meson decays and studies of $Z$ boson decays [17]. However, the large mass of the top quark, nearly $175 \mathrm{GeV} / c^{2}$, was in itself a surprise at the time. In this regard, the top quark separates itself from all other quarks: For example, it is the most massive fermion by a factor of nearly 40 (the bottom quark being the closest competitor). This peculiar characteristic of the top quark makes it behave very differently than any other quark, as will be described in Section 2.1 where we discuss the top quark phenomenology.

Interestingly, even though the top quark is the most recent quark observed, its mass is the best known of all quarks (with a relative uncertainty of $2.5 \%$ ). This is because it has such a short lifetime that it decays before any hadronization effects can occur, as discussed in more detail in Sec. 2.1. We demonstrate in Sec. 2.2 that we should not be satisfied with this relative success, and that a more accurate determination of $M_{t o p}$ is strongly motivated inside and beyond the SM. 


\subsection{Top Quark Phenomenology}

\subsubsection{General Picture of Top Quark}

The top quark is the weak isospin partner of the b-quark in the Standard Model. As such, it carries the following quantum numbers: an electric charge $+2 / 3$, an intrinsic spin of $1 / 2$ and a color charge associated with the strong force. Due to the relatively small data sample collected in Run I of the Tevatron, none of these assignments have been measured directly. However, strong indirect evidence exists. First, the precision electroweak data (discussed further in Sec. 2.2.1) of $Z$ boson decay properties requires the existence of an isospin partner of the $b$-quark with electric charge $+2 / 3$ and a large mass. Furthermore, the predicted rate of top quark pair production (discussed in Sec. 2.1.2), which is very sensitive to the spin and strong coupling of the top quark, is in good agreement with the data $[19,20,21,22]$. Therefore, current observations lead us to believe that the particle observed at the Tevatron is indeed the top quark. However, direct measurements are still desirable and will be attempted in the case of the electric charge and spin using data from the Run II of the Tevatron or the LHC, as discussed in more detail in [23].

The other intrinsic properties of an elementary particle are its mass and lifetime. The most precise knowledge of the mass comes from direct measurements. The current world average containing only measurements performed at the Run I of the Tevatron is $178.0 \pm 4.3 \mathrm{GeV} / c^{2}[6]$. In quantum mechanics, the lifetime $\tau$ of a particle is related to its natural width $\Gamma$ through the relationship $\tau=\hbar / \Gamma$. As discussed further in Sec. 2.1.3, the branching fraction for the electroweak top quark decay $t \rightarrow W b$ is far larger than any other decay mode and thus its full width can be approximately calculated from the partial width $\Gamma(t \rightarrow W b)$. Assuming $M_{W}=M_{b}=0$, the lowest order calculation of the partial width yields

$$
\Gamma_{0}(t \rightarrow W b)=\frac{G_{F} M_{t o p}^{2}\left|V_{t b}\right|^{2}}{8 \pi \sqrt{2}}=1.76 \mathrm{GeV},
$$

where $G_{F}$ is the Fermi constant and $V_{t b}$ is the Cabibbo-Kobayashi-Maskawa (CKM) 
matrix element linking the top and bottom quarks. This simplified picture illustrates that the width is driven by the square of $M_{t o p}$. More sophisticated calculations result in negative corrections of about $-20 \%$, the final result being $1.42 \mathrm{GeV}$ with small theoretical uncertainties $(<1 \%)$. This results in a lifetime for the top quark of approximately $4 \times 10^{-25} \mathrm{~s}$. This is about an order of magnitude lower than the characteristic time for Quantum Chromodynamics (QCD) effects to take place. Therefore, due to its very large mass, the top quark will not form hadrons before it decays. This property of the top quark has important phenomenological consequences. Indeed, the top quark is the only quark without hadron spectroscopy, i.e. where we can expect meson or baryon states including a top quark. In addition, the short lifetime facilitates the measurement of top quark properties (like its mass) since the information about the bare quark is directly reflected by the decay products.

\subsubsection{Top Quark Production}

The top quark is produced predominantly in top-antitop pairs $(t \bar{t})$ at the Tevatron via the strong interaction. At a center-of-mass energy $(\sqrt{s})$ of $1.96 \mathrm{TeV}$, the processes $q \bar{q} \rightarrow t \bar{t}$ and $g g \rightarrow t \bar{t}$ occur approximately $85 \%$ and $15 \%$ of the time, respectively. The leading order diagrams for the two processes are shown in Fig. 2.1. Calculations of the total $t \bar{t}$ cross-sections $\sigma(t \bar{t})$ have been performed up to the next-to-leading order (NLO) in the coupling constant of the strong force $\left(\alpha_{s}\right)$. The current theoretical value for $\sigma(t \vec{t})$ at a center-of-mass energy of $1.96 \mathrm{TeV}[18]$ is

$$
\sigma_{\text {theo }}(t \bar{t})=6.7_{-0.9}^{+0.7} \mathrm{pb}
$$

for $M_{t o p}=175 \mathrm{GeV} / c^{2}$. Since the typical partonic center-of-mass energy available at the Tevatron is still relatively close to the $t \bar{t}$ threshold production, (for instance the average velocity of the produced top quarks is $\beta \approx 0.5$ ), the cross section displays significant dependence on $M_{t o p}$. This is illustrated in Fig. 2.2 where we show $\sigma(t \bar{t})$ as a function of the 


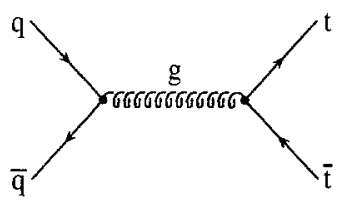

(a)
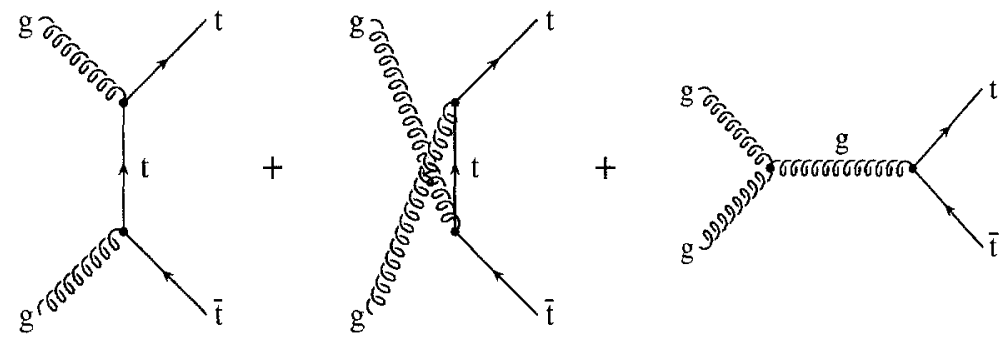

(b)

Figure 2.1: Tree level Feynman diagrams for the strong interactions (a) $q \bar{q} \rightarrow t \bar{t}$ and (b) $g g \rightarrow t \bar{t}$.

center-of-mass energy for various values of $M_{\text {top }}$. The theoretical calculations are in good agreement with measurements performed at $\sqrt{s}=1.8 \mathrm{TeV}$ (Run I) $[19,20]$ and $\sqrt{s}=1.96$ $\mathrm{TeV}$ (Run II) [21, 22]. Figure 2.2 illustrates one motivation to measure accurately $M_{t o p}$ : The knowledge of the top quark mass is necessary to compare as precisely as possible the theoretical predictions and measurements of the $t \bar{t}$ cross-section. An eventual discrepancy could be a sign of new physics as discussed in more detail in [23].

The electroweak production of single top quarks is also predicted by the Standard Model but has not been observed to date [24]. The production cross-section is predicted to be smaller than for $t \bar{t}(\sim 2.4 \mathrm{pb})$ and the experimental signature suffers from much larger background contamination.

\subsubsection{Top Quark Decay}

The top quark decay is mediated by the electroweak interaction. Since flavor changing neutral currents are forbidden in the Standard Model due to the GIM mechanism [25], 


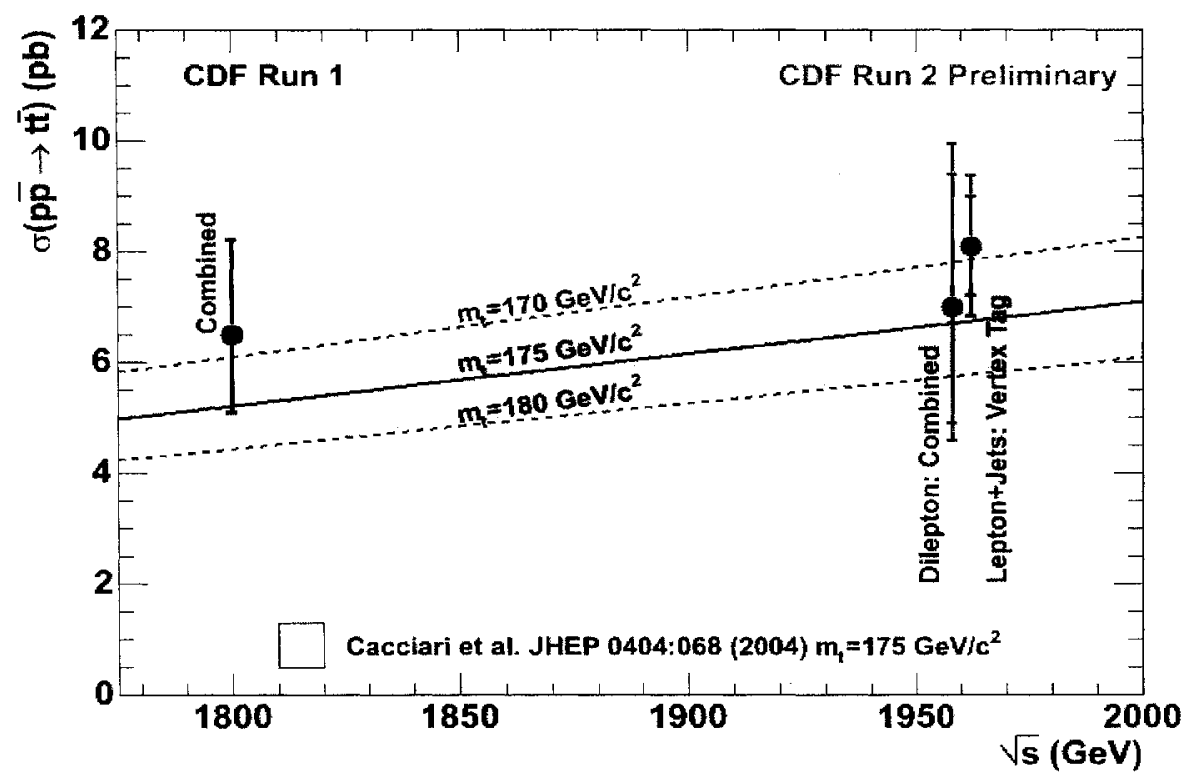

Figure 2.2: Cross-section of $t \bar{t}$ pair production as a function of $\sqrt{s}$ for the theory prediction [18] and CDF measurements [20, 21, 22].

the decays of the top quark involving $Z$ or $\gamma$ bosons in the final state, e.g. $t \rightarrow Z c$, are highly suppressed and can only occur through higher order diagrams. Therefore, the top quark decay vertex must include a $W$ boson. Three possible final states exist: $t \rightarrow W b$, $t \rightarrow W s$, and $t \rightarrow W d$. As illustrated in Eqn. 2.1, the partial width of charged current top decays is proportional to the square of the corresponding CKM matrix element. Assuming a Standard Model with three families, the relevant CKM matrix element have the following constraints [5]:

$$
\begin{aligned}
0.0048 & <\left|V_{t d}\right|<0.014 \\
0.037 & <\left|V_{t s}\right|<0.043 \\
0.9990 & <\left|V_{t b}\right|<0.9992 .
\end{aligned}
$$

Therefore, the decay $t \rightarrow W b$ is completely dominant and its predicted branching ratio is $B R(t \rightarrow W b)>99.8 \%$. Hence only $t \rightarrow W b$ decays have been considered in the 


\begin{tabular}{c|c}
\hline \hline Channel & Branching ratio \\
\hline All-hadronic & $46 \%$ \\
\hline Lepton+jets & $29 \%$ \\
\hline Dilepton & $5 \%$ \\
\hline Tau lepton+anything & $20 \%$ \\
\hline \hline
\end{tabular}

Table 2.1: Branching ratios of the $t \bar{t}$ experimental signatures.

identification of top quarks, though searches for other decay modes have been undertaken [26]. We note that the $W$ boson from the top quark decay is real, i.e. its mass corresponds to the measured mass $M_{W} \approx 80.4 \mathrm{GeV} / c^{2}$, given that $M_{\text {top }}>M_{W}+M_{b}$. This is an important characteristic of $t \bar{t}$ events that is exploited in our analysis in the reconstruction of the top quark mass (see Chapter 5) and the $W$ boson mass (see Chapter 6). The $W$ boson will in turn decay to two quarks approximately $2 / 3$ of the time and a charged lepton associated with a neutrino about $1 / 3$ of the time.

The experimental signature of top quarks thus emerge. They are produced as topantitop pairs, each one decaying immediately to a real $W$ boson and a $b$-quark, the latter hadronizing to form a $b$-jet. The resulting $W$ boson decays define the $t \bar{t}$ final state: There can be two hadronic decays (all-hadronic channel), one leptonic and one hadronic decay (lepton+jets channel), and two leptonic decays (dilepton channel), where the leptonic decays considered are usually only to electrons or muons (with their associated neutrinos) due to the experimental difficulty of identifying tau leptons. The approximate branching ratios for each channel are given in Tab. 2.1.

The measurement described in this dissertation uses the leptontjets channel. It suffers from less background than the all-hadronic channel (dominated by QCD multijets events) and the events can be fully reconstructed, in comparison to the dilepton channel where the event reconstruction is complicated by the presence of two neutrinos that are not directly detectable. Historically, the lepton+jets channel has yielded the best 


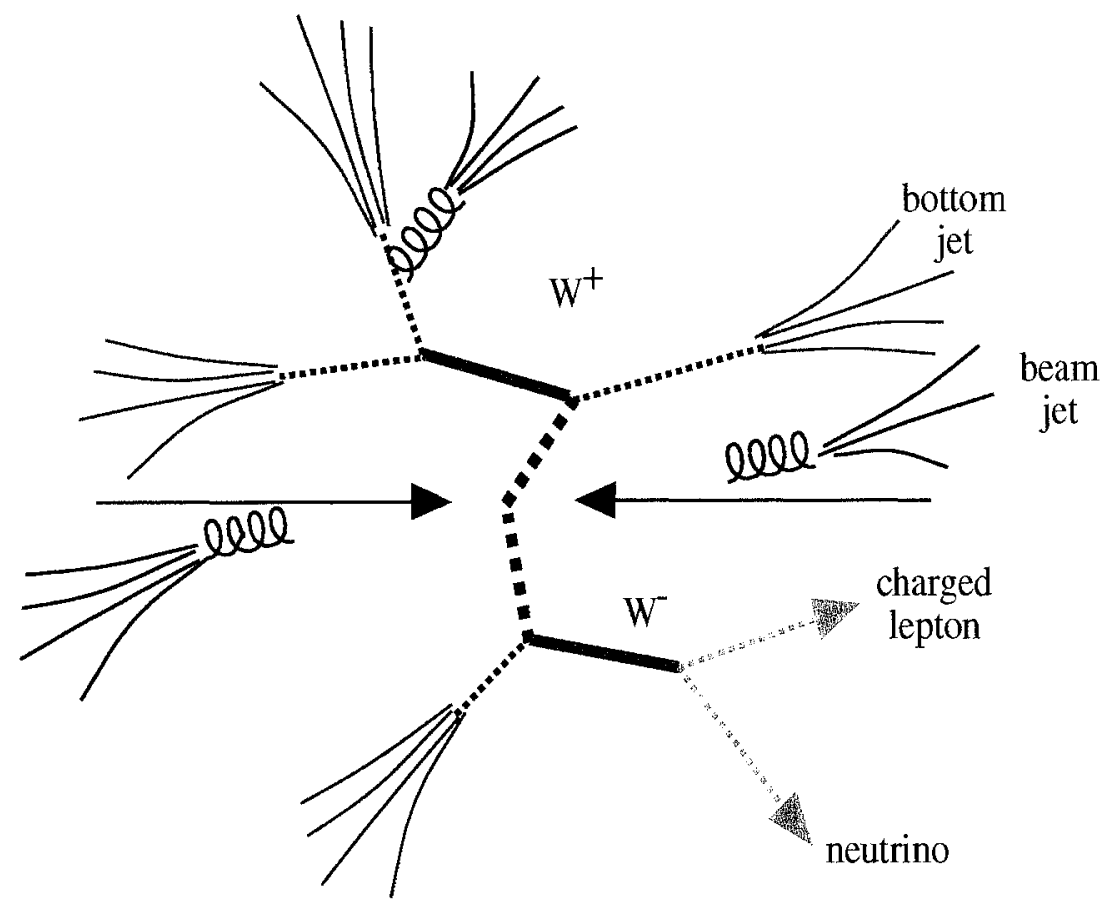

Figure 2.3: Diagram of a lepton+jets $t \bar{t}$ event: two partons inside the proton and antiproton (illustrated by the arrows) collide to produce a $t \bar{t}$ pair. Each top quark decays to a $W$ boson and a $b$ quark. One $W$ boson decays to two quarks while the other decays to a lepton and its associated neutrino. Finally, each quark of the final state fragments to form a jet.

measurements of top quark properties. A sketch of a lepton+jets event is shown in Fig. 2.3.

\subsection{Motivation for a Precise Top Quark Mass Mea- surement}

In this section we review the motivation to measure $M_{\text {top }}$ precisely. We describe first the role of the top quark in precision electroweak analyses of parameters. This is followed by a discussion of the importance of measuring $M_{t o p}$ in possible alternative scenarios to the SM. 

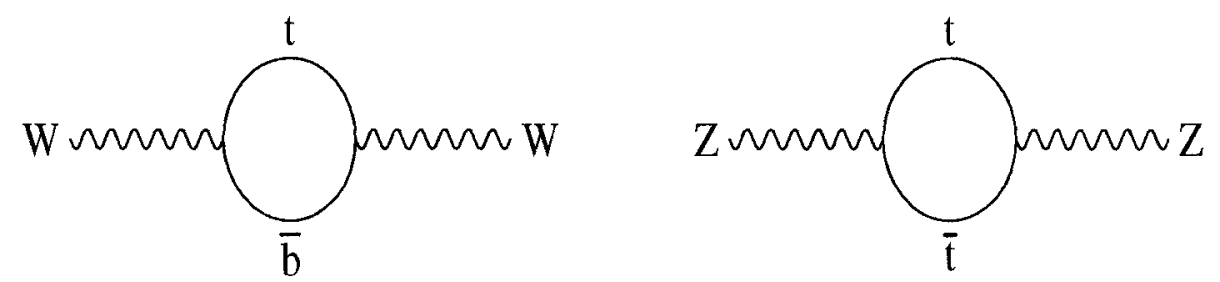

Figure 2.4: The propagators of the $W$ and $Z$ bosons where a loop involving top quarks is contributing.

\subsubsection{Precision Electroweak fits}

As an effective scientific theory, the Standard Model can make predictions that are verifiable by experiments. Many tests of the SM have been performed in the last few decades (a current status of these tests can be found in [5]). No significant discrepancies between theory and experiment have been observed so far, which confirms the success of the SM to explain particle physics up to the energy scale of a few hundred GeV available at current or past colliders. However, further precision in both the theoretical calculations and experimental analyzes are desirable. Indeed, the Standard Model is considered incomplete, at least beyond the $\mathrm{TeV}$ level, as will be discussed further in Sec. 2.2.2, and signs of new physics could appear in the form of radiative corrections (e.g. internal loops of Feynman diagrams) to precision observables.

The top quark plays a central role in the predictions of many SM observables by contributing to their radiative corrections. Good examples are the $W$ and $Z$ boson propagators, in which loops involving top quarks are expected to strongly contribute, as illustrated in Fig. 2.4. These diagrams can exist for any type of quark or lepton, but the very large value of $M_{t o p}$ makes the top quark contribution dominant. To illustrate the effect of the top quark, we consider the theoretical calculation of the $W$ boson mass [5] :

$$
M_{W}^{2}=\frac{\pi \alpha}{\sqrt{2} G_{F} \sin ^{2} \theta_{W}} \frac{1}{1-\Delta r}
$$

where $\alpha$ is the fine structure constant, $\theta_{W}$ is the Weinberg angle and $\Delta r$ contains the 
radiative corrections and is approximately given by

$$
\Delta r \sim \Delta r_{0}-\frac{\Delta \rho}{\tan ^{2} \theta_{W}}
$$

The term $\Delta r_{0}$ is due to the running of $\alpha$ and the term $\Delta \rho$ is due to the one-loop top quark correction to $W$ boson propagators illustrated in Fig. 2.4, and is given by ${ }^{1}$

$$
\Delta \rho=\frac{3 G_{F} M_{t o p}^{2}}{8 \sqrt{2} \pi^{2}}
$$

The uncertainty on the Fermi constant $G_{F}$ is completely negligible with respect to the one on the top quark mass in the computation of $\Delta \rho$. The term $\Delta r_{0}$ and the Weinberg angle in Eqn. 2.7 are known to a precision of $0.2 \%$. The uncertainty on the top quark mass is currently about an order of magnitude larger than the other uncertainties and moreover it contributes quadratically to $\Delta r$. Thus the precision on $M_{\text {top }}$ is currently the limiting factor in the theoretical prediction of the $W$ boson mass. The parameter $\Delta \rho$ is qualified as "universal" in the literature because it enters in the calculation of many other electroweak observables like $\sin \theta_{W}$ and the ratio of the production of $b$ quark hadrons to hadrons of all types (usually denoted as $R_{b}$ ), to name a few. Therefore the top quark mass plays a central role in the interplay between theoretical predictions and experimental observables that aims to test the consistency of the SM.

One consistency check is to compare the measured value of $M_{\text {top }}$ with the predicted value from SM precision observables (excluding of course direct measurements of $M_{t o p}$ ). The indirect constraints, inferred from the effect of top quark radiative corrections, yields $M_{t o p}=181_{-9}^{+12} \mathrm{GeV} / c^{2}[15]$. The relatively small uncertainty is achieved because of the large dependence of $M_{\text {top }}$ on many electroweak observables. This is in remarkable agreement with the Run I world average of $M_{t o p}=178.0 \pm 4.3 \mathrm{GeV} / c^{2}[6]$, and is considered a success of the SM.

A similar procedure can be used to constrain the Higgs boson mass $\left(M_{H}\right)$, the last particle in the SM that has yet to be observed. The only direct information on $M_{H}$ is

\footnotetext{
${ }^{1} \mathrm{~A}$ derivation of $\Delta \rho$ can be found in [17].
} 
a lower bound obtained from searches at LEP-II: $M_{H}>114 \mathrm{GeV} / c^{2}$ at $95 \%$ confidence level [27]. Indirect constraints on $M_{H}$ can be obtained with precise measurements of $M_{W}$ and $M_{\text {top }}$. Indeed, the correction to the $W$ boson mass $\Delta r$ given in Eqn. 2.7 contains additional terms due to Higgs boson loops. These corrections depend only logarithmically on $M_{H}$ and have thus weaker dependence on $M_{H}$ than on $M_{t o p}$. Still, precise determinations of $M_{t o p}$ and $M_{W}$ can be used to obtain meaningful constraints on $M_{H}$ as illustrated in Fig. 2.5. Numerically, the constraints are [15]

$$
\begin{aligned}
& M_{H}=126_{-48}^{+73} \mathrm{GeV} / c^{2} \\
& M_{H}<280 \mathrm{GeV} / c^{2} \text { at } 95 \% \text { C.L. }
\end{aligned}
$$

where only the top quark mass measurements from Run I have been used. Such constraints on $M_{H}$ can help direct future searches at the Tevatron and LHC and constitutes another stringent test of the Standard Model when compared to limits from direct searches or mass measurements from an eventual discovery.

\subsubsection{The Top Quark Beyond the Standard Model}

Even though the Standard Model successfully describes experimental data up to a few hundred $\mathrm{GeV}$, it is believed that new physics must come into play at some greater energy scale. At the very least, gravity effects are expected at the Planck scale $\left(\sim 10^{19} \mathrm{GeV}\right)$ that the SM ignores in its current form.

The SM can thus be thought of as an effective theory with some unknown new physics existing at a higher energy scale. A link exists between the new physics and the SM that manifests itself through radiative corrections to SM particles. The Higgs boson sector is the most sensitive to loops of new particles. For example the Higgs boson mass corrections from fermion loops shown in diagram (a) of Fig. 2.6 are given by:

$$
\Delta M_{H} \sim-2 \Lambda^{2}+6 m_{f}^{2} \ln \left(\Lambda / m_{f}\right)+\ldots
$$


where $m_{f}$ is the fermion mass and $\Lambda$ is the "cut-off" scale used to regulate the loop integral. The parameter $\Lambda$ can be interpreted as the scale for new physics that typically corresponds to the scale of the Grand Unified Theory (GUT) near $10^{16} \mathrm{GeV}$. This is a problem for the SM, since on the one hand the Higgs boson mass receives corrections of the order of $10^{16} \mathrm{GeV}$ from new physics, while on the other hand it is required to be of the order of $100 \mathrm{GeV} / c^{2}$ to give the correct mass to the SM electroweak gauge bosons. There is a discrepancy of 14 orders of magnitude between the targeted mass and the radiative corrections! This is known as the fine-tuning problem of the SM Higgs boson (or gauge hierarchy problem) and has occupied theoretical physicists for several decades. A few solutions have emerged from this work, all of them manifesting themselves near the scale of the origin of mass near $1 \mathrm{TeV}$ (or electroweak symmetry breaking scale).

The top quark, with its large mass of nearly $0.2 \mathrm{TeV}$, could be more closely connected to new physics than any other SM particle. One interesting numerological argument suggests the top quark is indeed a special particle. Its Yukawa coupling $\left(y_{t}\right)$, i.e. its coupling to the Higgs field, is approximately equal to unity:

$$
y_{t}=\sqrt{2} \frac{M_{t o p}}{v} \approx 1
$$

where $v$ is the vacuum expectation value of the Higgs field that is known from properties of the weak interaction to be approximately $174 \mathrm{GeV}$. This could be a coincidence, or it could be a sign that the top quark mass is related to the mechanism of the origin of mass that physics beyond the SM must explain, as suggested above. In this respect, the top quark mass could turn out to be a more fundamental parameter of nature. For these reasons, albeit somewhat hypothetical, a precise measurement of $M_{t o p}$ would certainly be desirable for the understanding of any new theory.

One example of a new physics model is Supersymmetry (SUSY), which constitutes an extension of the SM where the SM fermionic particles have associated bosonic particles 
and vice-versa. ${ }^{2}$ It is generally regarded as the favored option to extend or replace the SM at higher energies. Indeed, SUSY solves elegantly the gauge hierarchy problem since the fermion and boson partners cancel each other's divergent corrections to the Higgs boson mass proportional to $\Lambda^{2}$ (given in Eqn. 2.11 for fermionic particles). Moreover, SUSY has other attractive features, such as providing a good candidate for dark matter, predicting the unification of the gauge coupling constants at the GUT scale and being required by the only consistent theory of quantum gravity currently available (superstring theory).

The top quark plays an important role in SUSY models. Indeed, the radiative corrections from SUSY particles to electroweak observables, which can be computed in a similar fashion as for the SM particles, are dominated by loops involving the top quark and its scalar partners, the stop quarks. This effect is especially apparent in the Higgs sector of SUSY models. Considering the simplest model of SUSY, the Minimum Supersymmetric Standard Model (MSSM), the one-loop correction to the lightest MSSM Higgs boson $\operatorname{mass}^{3}\left(M_{h}\right)$ is proportional to [29]

$$
\Delta M_{h}^{2} \sim G_{F} M_{t o p}^{4} \log \left(\frac{M_{\tilde{t_{1}}} M_{\tilde{t_{2}}}}{M_{t o p}^{2}}\right),
$$

where $M_{\tilde{t_{1}}}$ and $M_{\tilde{t_{2}}}$ are the mass of the lightest and heaviest stop quarks, respectively. Thus the corrections to $M_{h}$ depend quartically on $M_{t o p}$ ! Therefore, the same conclusion as discussed previously for the SM is valid for SUSY (and even reinforced due to the stronger $M_{t o p}$ dependence): high precision measurements of $M_{t o p}$ will be crucial for the self-consistency check of the theory and determination of unknown parameters. For instance, the value of the top quark mass was crucial to determine the current upper bound of about $135 \mathrm{GeV} / c^{2}$ on the lightest MSSM Higgs boson mass [30].

Using the current measurements of precision observables, it is already possible to set meaningful constraints on SUSY. For example, Fig. 2.7 [31] shows the current measurements of $M_{t o p}$ and $M_{W}$ as well as the region allowed exclusively inside the MSSM

\footnotetext{
${ }^{2}$ An exhaustive review of Supersymmetry can be found in [28].

${ }^{3} \mathrm{~A}$ minimum of five physical Higgs bosons are required in SUSY models.
} 
(green), the SM (red) as well as an overlap region between the MSSM and SM (blue). As can be seen, the additional radiative corrections from SUSY particles are large enough such that the overlap region between SM and MSSM is small in the $M_{t o p}-M_{W}$ plane. The current experimental accuracies are not good enough to distinguish between the two theories, but future prospects (e.g. black curve for Tevatron/LHC and red curve for the International Linear Collider (ILC)) demonstrates very good discriminating power. The radiative corrections from MSSM particles to the SM precision observables are discussed in more detail in $[31,32]$.

Other alternatives to replace the SM at energies near the $\mathrm{TeV}$ scale are theories involving dynamical breaking of the electroweak symmetry [33]. These models, one wellknown example being Technicolor [34], do not include an elementary Higgs boson but rather give mass to the SM particles by introducing a new strong gauge interaction that produce condensates of fermions that act as Higgs bosons. In some versions of these models, denoted as "topcolor", the new gauge interaction acts only on the third generation, and the fermion condensates are made of top quarks [35]. Such a model could be discovered by looking for the evidence of new particles in the $t \bar{t}$ invariant mass at the Tevatron or LHC.

This summary of the role of the top quark in physics beyond the SM is not extensive and a more complete review can be found in [36]. 


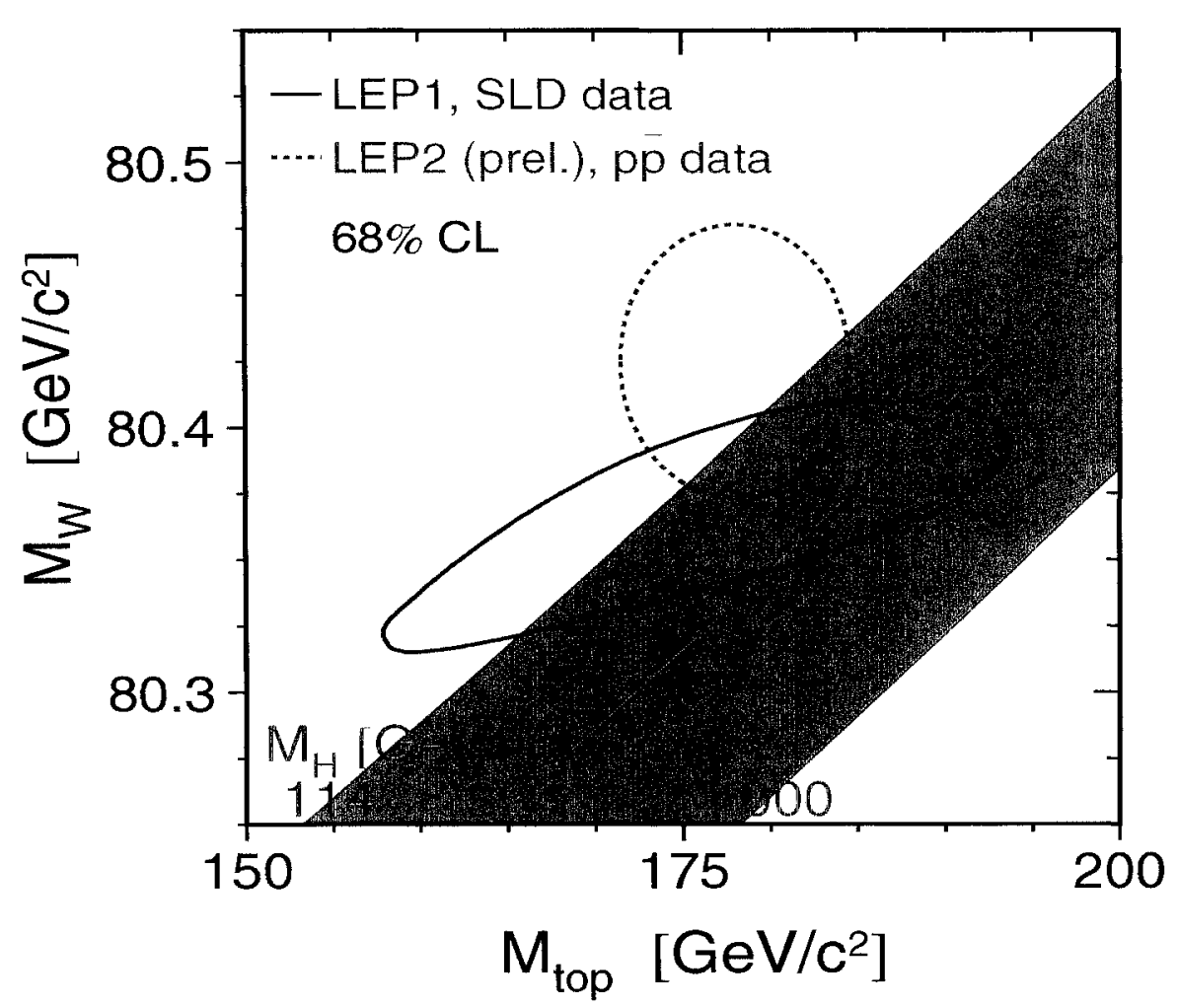

Figure 2.5: Constraint on the Higgs boson mass as a function of the top quark and $W$ boson measured masses as of spring of 2005 [15]. The full red curve shows the constraints (68\% C.L.) coming from studies at the $Z$ boson pole. The dashed blue curve shows constraints (68\% C.L.) from precise measurement of $M_{W}$ and $M_{t o p}$ (using Tevatron Run I data for $\left.M_{t o p}\right)$.

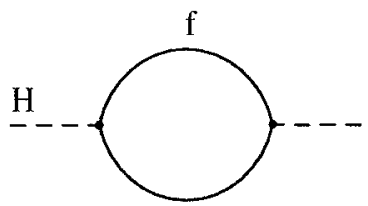

(a)

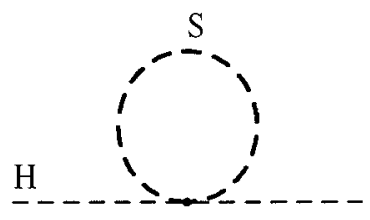

(b)

Figure 2.6: Loop contributions to the Higgs boson propagator from (a) fermionic and (b) scalar particles. 


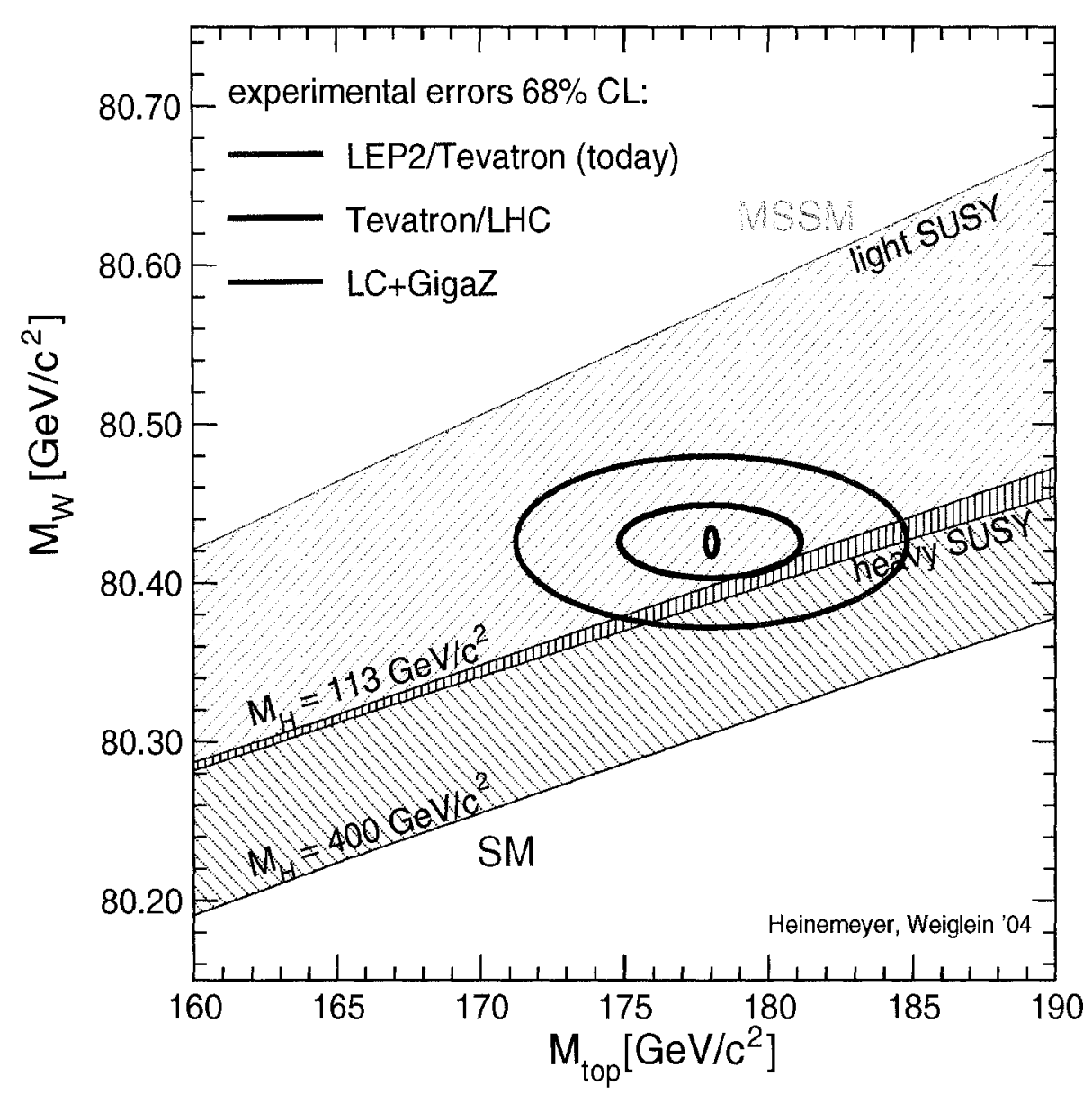

Figure 2.7: Experimental constraints on $M_{W}$ and $M_{\text {top }}$ (using Run I world average) (outer blue ellipse), the projected constraints at the end of the Tevatron and LHC (middle black ellipse) and at the International Linear Collider (ILC) (red inner ellipse). Also shown are the allowed region for MSSM (green hatched), the SM (red cross-hatched) and the overlap region between the SM and MSSM (blue vertical lines) [31]. 


\section{Chapter 3}

\section{Experimental Apparatus}

This measurement is performed by identifying and reconstructing $t \bar{t}$ pairs decaying to the final state $l \nu q \bar{q}^{\prime} b \bar{b}$. The top-antitop pairs are produced by the Tevatron accelerator located at the Fermi National Accelerator Laboratory in Illinois. The data sample is collected by CDF, a general purpose detector set on the Tevatron ring. In this chapter we describe the experimental apparatus employed to create and detect the top-antitop pairs. We also describe the simulation tools that are used in this analysis to model the signal and background events and their signature in the CDF detector.

\subsection{The Fermilab's Accelerator Chain}

The Tevatron is a superconducting synchrotron colliding proton and antiprotons with a center of mass energy of $1.96 \mathrm{TeV}$. In this section we describe the chain of accelerators used to create the colliding proton and antiproton beams and accelerate them to the desired energy. The process is driven by four systems, the proton source, the Main Injector, the antiproton source and the Tevatron. A sketch of the accelerator chain is shown in Fig. 3.1. 


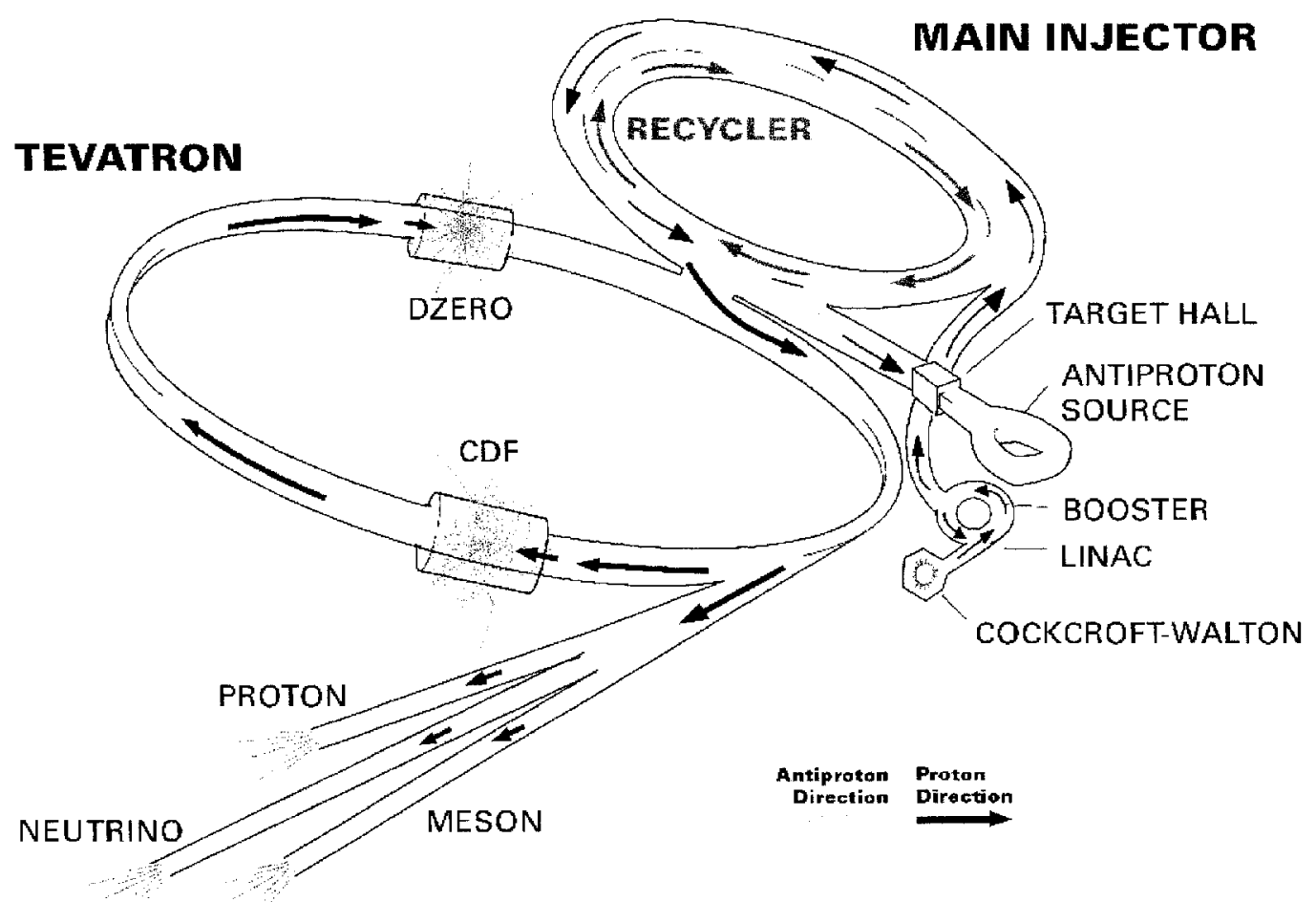

Figure 3.1: A diagram of the Fermilab's accelerator chain.

\subsubsection{The Proton Source}

The first step in the chain is the Cockcroft-Waltron electrostatic accelerator in which a gas of hydrogen is ionized by the addition of an electron. The resulting ions are accelerated up to an energy of $750 \mathrm{keV}$. The hydrogen ions then enter the Linac, a $130 \mathrm{~m}$ linear accelerator in which a series of radio-frequency (RF) cavities accelerate the ions up to $400 \mathrm{MeV}$. Carbon foils are positioned at the end of the Linac to remove both electrons from the hydrogen ions. The resulting protons are further accelerated by the Booster, a synchrotron accelerator with a circumference of $475 \mathrm{~m}$. The protons travel around the Booster ring approximately 16,000 times at which point they reach an energy of $8 \mathrm{GeV}$. The protons are then transfered to the Main Injector. 


\subsubsection{The Main Injector}

The Main Injector is a synchrotron with a circumference of about $3 \mathrm{~km}$ with the functions of (1) accelerating $8 \mathrm{GeV}$ protons from the booster up to $150 \mathrm{GeV}$, (2) accelerate protons to $120 \mathrm{GeV}$ and send them to the Target Station (described in Sec. 3.1.3), (3) accelerate $8 \mathrm{GeV}$ antiprotons from the antiproton source up to $150 \mathrm{GeV}$ and (4) inject the $150 \mathrm{GeV}$ protons and antiprotons into the Tevatron.

\subsubsection{The Antiproton Source}

Protons with an energy of $120 \mathrm{GeV}$ coming from the Main Injector are smashed on a nickel target every 1.5 seconds in the Target Station. The resulting particles, produced at different angles and momenta, are focused into a beam of particles using a series of magnets. The flow of particles from the target contains relatively few antiprotons $(\bar{p})$ : about $20 \bar{p}$ per million protons sent to target. Therefore, a magnet must be used to separate the antiprotons from the other particles with different mass or charge. The antiprotons are sent to the Debuncher, a synchrotron used to reduce the spread in energy of the antiprotons, making the beam easier to manipulate in the following acceleration steps. This is performed by synchronizing the oscillating potential in the RF cavities such that slower $\bar{p}$ traveling in the Debuncher ring are accelerated and vice-versa for faster $\bar{p}$. Next in the antiproton journey is the Accumulator, a storage ring used to collect antiprotons until an amount sufficient to produce $p \bar{p}$ collision at the desired luminosity is reached (typically $1.3 \times 10^{12} \bar{p}$ ). The accumulation rate is about $7 \times 10^{10} \bar{p}$ per hour and thus about 15 hours are needed to collect an adequate amount. The longitudinal momentum spread of the beam is minimized and its transverse momentum is reduced by using a combination of RF cavities and stochastic cooling [37] systems. When a sufficient number of antiprotons is collected, the beam is transfered to the Main Injector. 


\subsubsection{The Tevatron}

The Tevatron is a synchrotron with a circumference of about $6.3 \mathrm{~km}$. It uses approximately 1,000 superconducting dipole magnets, each one producing a field of $4.2 \mathrm{~T}$, to bend the beams around the ring. The protons and antiprotons are injected into the Tevatron where they are accelerated to $980 \mathrm{GeV}$. The beams travel around the ring, organized in 36 bunches of protons and antiprotons, and meet each other at several interaction points in the ring. The same injection of protons and antiprotons, referred to as a store, produce collisions for up to 30 hours in normal running conditions. The instantaneous luminosity of the collisions produced in the CDF detector ${ }^{1}$ at the beginning of a store varies depending on the data taking period and is shown in Fig. 3.2 for the first four years of running in Run II (started in June 2001). The Tevatron uses RF cavities to accelerate the $p$ and $\bar{p}$ and a series of electrostatic separators keep the beams apart between collisions points. The proton and antiproton bunches contain about $2.6 \times 10^{11}$ and $3.5 \times 10^{10}$ particles, respectively, and cross at a given interaction point every $396 \mathrm{~ns}$. There is on average approximately $0.6 p \bar{p}$ interactions per bunch crossing for the data sample used in this analysis.

\subsection{The CDF-II Detector}

The Run II CDF detector (CDF-II) is designed to study many aspects of particle physics at the energy frontier provided by the Tevatron: the physics program of CDF includes studies of the bottom and top quarks, electroweak and quantum chromodynamics physics as well as the search for new phenomena. It is thus a general-purpose detector with considerable flexibility and complexity.

A tracking system is positioned closest to the beamline to provide accurate momentum determination of charged particles. The tracking system is immersed in a $1.4 \mathrm{~T}$ uniform

\footnotetext{
${ }^{1}$ The calculation of the instantaneous luminosity by the CLC detector is described in Sec. 3.2.5.
} 


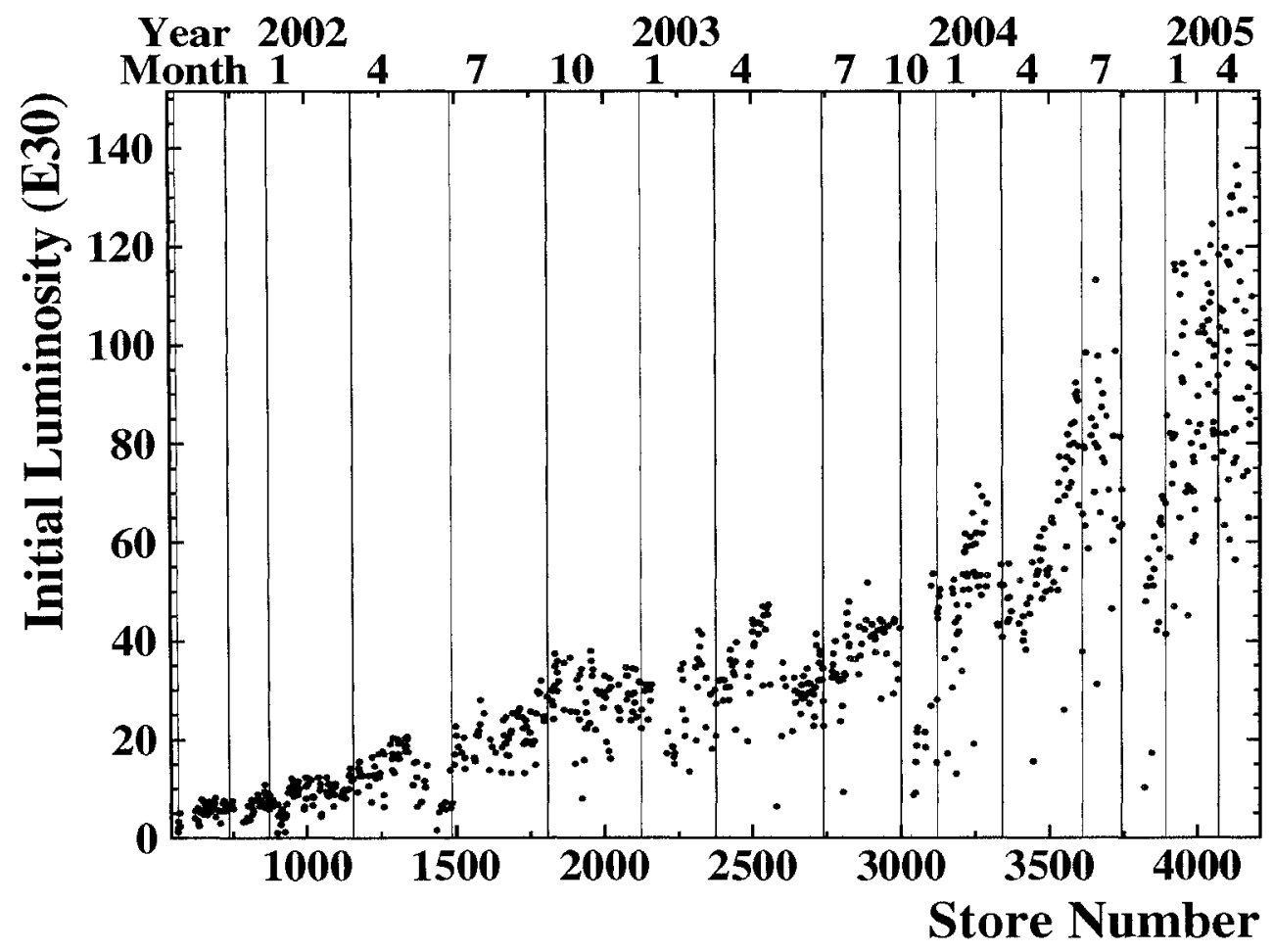

Figure 3.2: Initial instantaneous luminosity at CDF as a function of store number (bottom axis) and date of data taking (top axis) (June 2001 to June 2005).

magnetic field (aligned in the proton direction) produced by a superconducting solenoid $4.8 \mathrm{~m}$ long and positioned at a radius of $1.5 \mathrm{~m}$. Calorimeters located outside the tracking volume provide energy measurement of electrons, photons and jets. The geometrical coverage of the calorimeters is maximized to measure the energy flow of all particles produced in a collision and indirectly to measure the missing transverse energy resulting from the presence of weakly interacting particles like neutrinos. Muon chambers are located behind the calorimeters to detect the presence of muons. An elevation view of one-half of the CDF detector is shown in Fig. 3.3. We describe each of the subdetector systems in more detail in the following sections. The Reference [8] provides a detailed description of the detector.

The coordinate system for CDF is defined as follows: the $z$ axis is along the beamline in the direction of the protons (or equivalently the solenoid field lines), the $x$ axis is in 


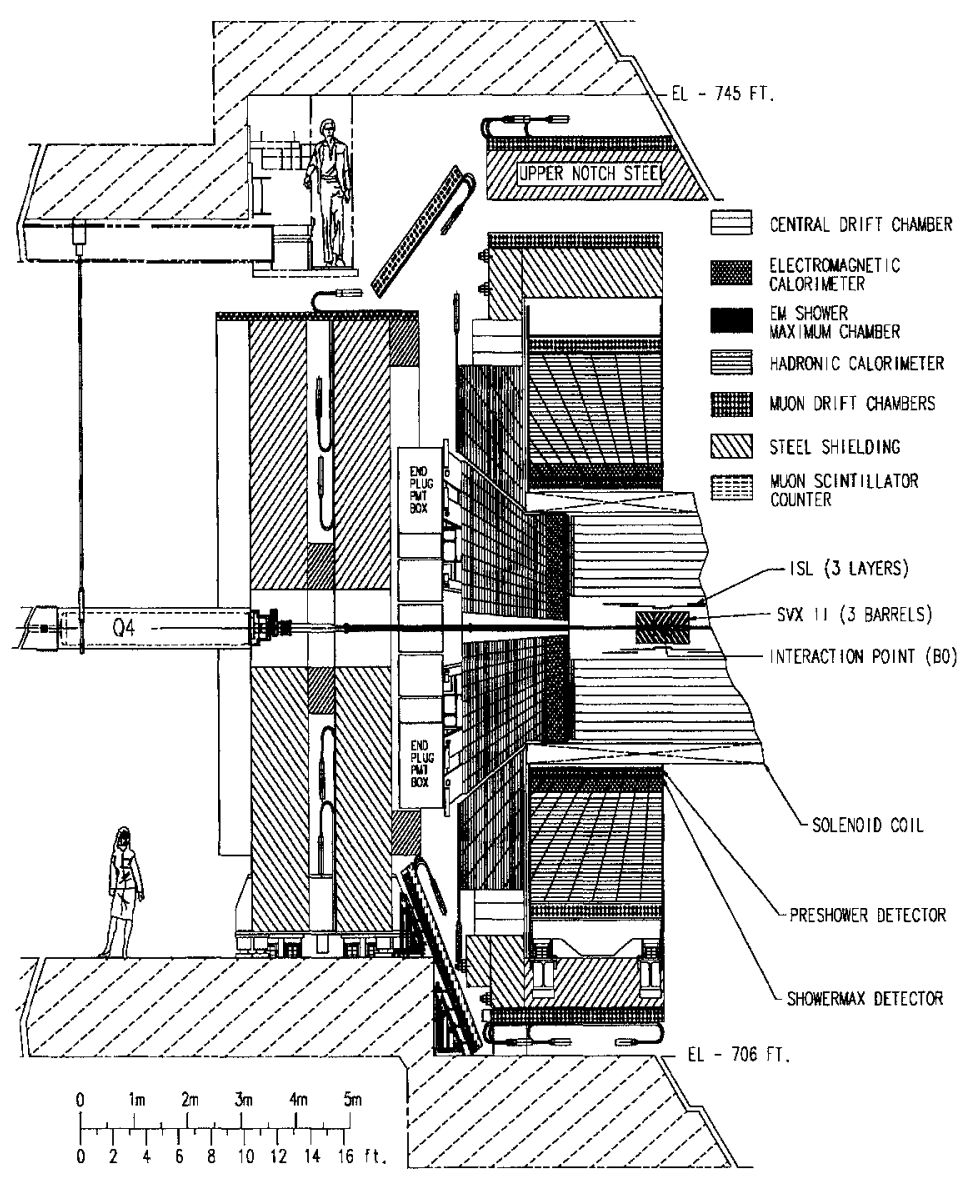

Figure 3.3: Elevation view of one-half of the CDF-II detector. The other half is mirror symmetric.

the same plane as the Tevatron ring and points outward, and the $y$ axis points straight up. Cylindrical coordinates $r, \phi$ and $\theta$ (defined from the cartesian coordinates above) are more natural to the CDF detector layout and more commonly used. The transverse momentum and energy are defined as $p_{T}=p \sin \theta$ and $E_{T}=E \sin \theta$, respectively. Since the center-of-mass energy is unknown at the partonic level in $p \bar{p}$ collisions, the polar angle is generally replaced by a well behaved variable under Lorentz transformations in the $z$ direction: the pseudorapidity ${ }^{2}$ defined as $\eta_{\text {det }}=-\frac{1}{2} \ln (\tan \theta)$. When the pseudorapidity is calculated relative to the actual interaction vertex location, it is simply denoted as $\eta$.

\footnotetext{
2 The pseudorapidity is the relativistic limit of the rapidity defined as $y=\frac{1}{2} \ln \left(\frac{E+p_{z}}{E-p_{z}}\right)$.
} 


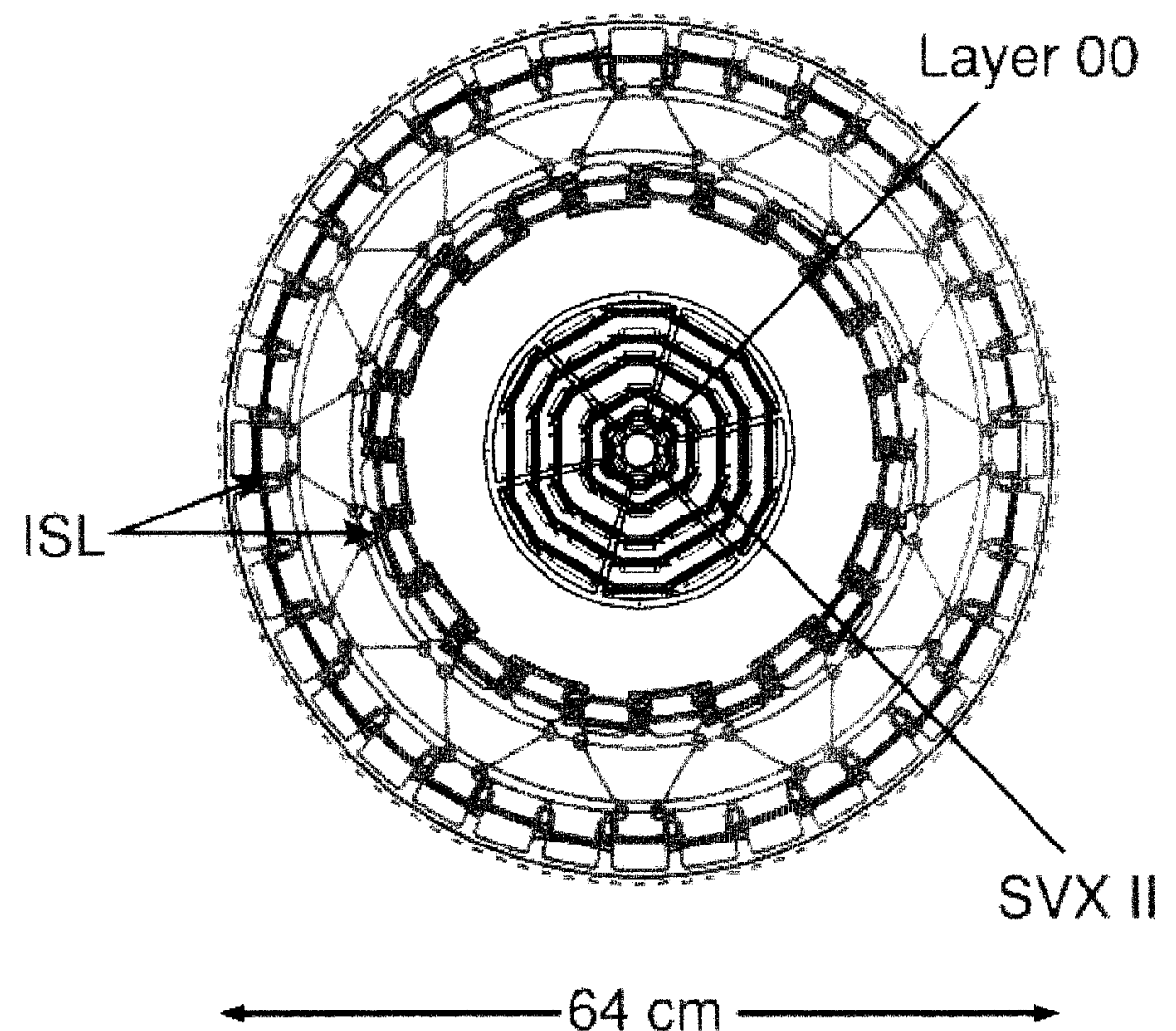

Figure 3.4: End view of the CDF-II silicon system.

\subsubsection{Silicon Detectors}

The tracking detectors can be separated into two systems: the silicon detectors and the Central Outer Tracker (described below). There are three sets of silicon detectors that have cylindrical shapes and are arranged in a concentric manner, L00, SVX and the ISL subdetectors. Their main purpose is to provide very high precision measurements of the position of charged particles near the $p \bar{p}$ interaction point. An end view of the silicon detectors is shown in Fig. 3.4. The silicon detectors cover the pseudorapidity region $\left|\eta_{\text {det }}\right| \lesssim 2$ as shown in the side view of the silicon detectors in Fig. 3.5.

The first detectors (L00) are a single-sided layer of silicon microstrip arrays with a readout pitch of $50 \mu \mathrm{m}$ that provide only axial measurements. This layer is mounted directly on the Tevatron beampipe $(r=1.6 \mathrm{~cm})$ to provide a measurement point as close 
to the beam as possible.

The Silicon Vertex Detector (SVX) is made of 5 concentric layers of silicon detectors arranged in three barrels in $z$. Each layer is arranged in 12 wedges of silicon strips in $\phi$ as illustrated in Fig. 3.4. It is $96 \mathrm{~cm}$ long and is located at radii ranging from $2.4 \mathrm{~cm}$ to $10.7 \mathrm{~cm}$. Each wedge is made of double-sided silicon detectors. One side of the strip provides an axial measurement. On three of the layers the other side have strips positioned at a stereo angle of $90^{\circ}$, providing information in the $z$ direction. The stereo strips on the remaining two layers are arranged at a small stereo angle of $1.2^{\circ}$, therefore providing information in both the $z$ and $\phi$ directions. The strip pitch is $55-65 \mu \mathrm{m}$ for axial strips, $60-75 \mu \mathrm{m}$ for $1.2^{\circ}$ stereo strips and $125-145 \mu \mathrm{m}$ for $90^{\circ}$ stereo strips. The SVX detector resolution for an axial measurement point is $\approx 12 \mu \mathrm{m}$.

The Intermediate Silicon Layers (ISL) are made of three layers of double-sided silicon detectors that are located at radii from $20 \mathrm{~cm}$ to $29 \mathrm{~cm}$. One side of the silicon detectors contains axial strips (with strip pitch of $55 \mu \mathrm{m}$ ) while the other side contains stereo strips at an angle of $1.2^{\circ}$ (with a strip pitch of $73 \mu \mathrm{m}$ ). The ISL measures the axial position of tracks with a resolution of $\approx 16 \mu \mathrm{m}$ and provides at least one additional measurement point in the central region $(|\eta|<1)$ between the SVX and the Central Outer Tracker (located at a radius outside of $40 \mathrm{~cm}$ ) and two additional measurement points in the forward region $(1<|\eta|<2)$.

\subsubsection{Central Outer Tracker}

The Central Outer Tracker (COT) is an open-cell drift chamber located at radii between $40 \mathrm{~cm}$ and $132 \mathrm{~cm}$. It provides 96 measurement points of each charged particle trajectory. The COT is $310 \mathrm{~cm}$ long and covers the pseudorapidity range $\left|\eta_{\text {det }}\right| \lesssim 1$.

The COT is arranged in 8 superlayers, each containing 12 layers of sense wires strung in alternation with potential wires. The drift cells are arranged such that the wires are positioned in the middle of two gold-on-mylar cathode planes that are separated by $2 \mathrm{~cm}$. 


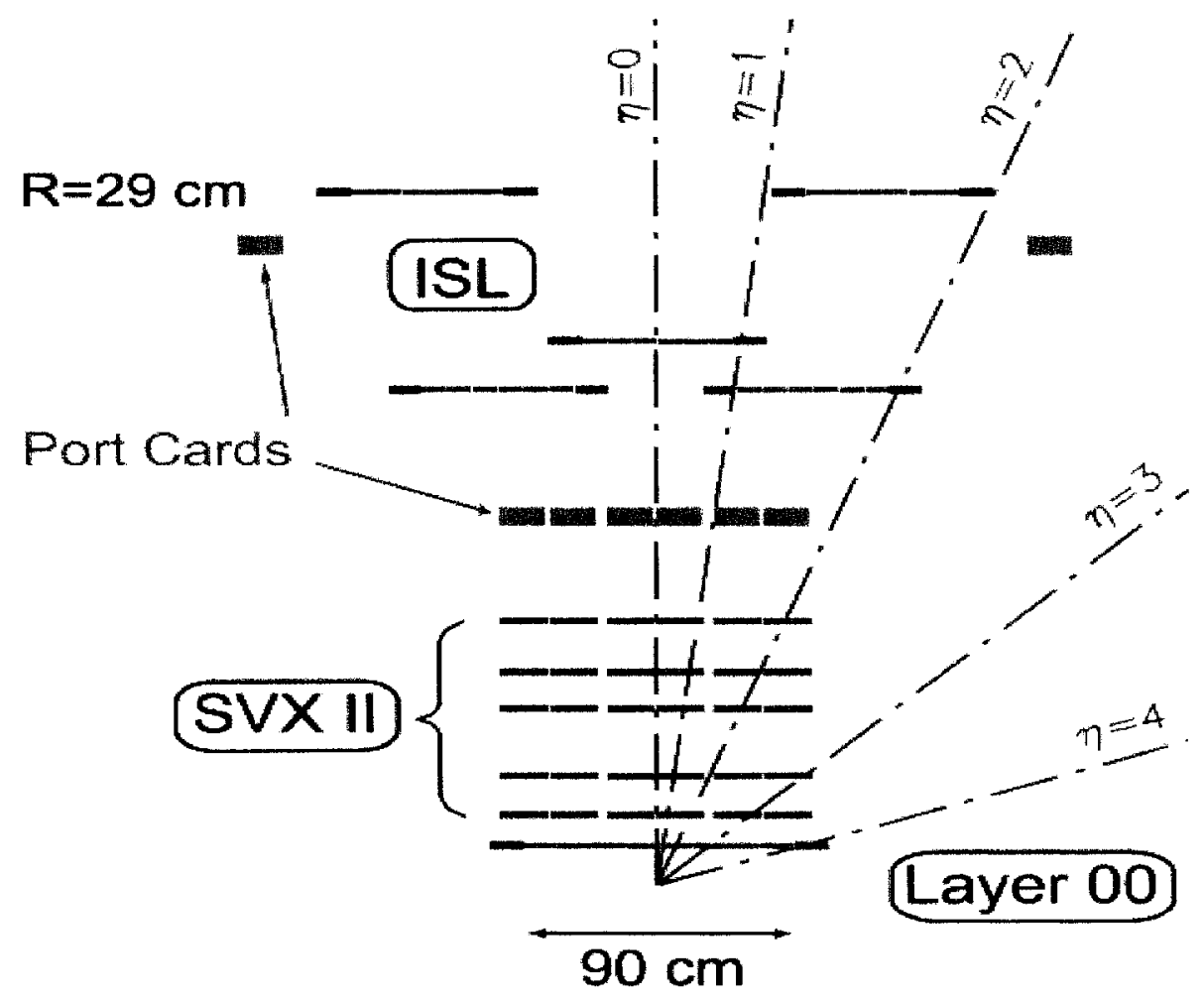

Figure 3.5: Side view of one half of the CDF-II silicon system.

The wires in half of the superlayers are strung parallel to the beamline, providing axial measurements. For the other four "stereo" superlayers, the wires are strung at small stereo angles of $\pm 2^{\circ}$ with the beamline, therefore providing stereo information of a hit position. Axial and stereo superlayers alternate, starting with a stereo superlayer at the inner radius.

The gas used in the COT is made of $50 \%$ argon and $50 \%$ ethane, which provides for a relatively high drift velocity $(\approx 100 \mu \mathrm{m} / \mathrm{ns})$. Given that the maximum drift distance is $0.88 \mathrm{~cm}$ in the COT, the maximum drift time is $100 \mathrm{~ns}$. That allows all of the hit signals on the wires to be read out between each Tevatron bunch crossing (occurring every $396 \mathrm{~ns})$.

The COT single-hit resolution has been measured in situ to be $180 \mu \mathrm{m}$ using $\mathrm{Z}$ boson decays into muon pairs, $Z \rightarrow \mu \mu$. Hits are combined together to form track 
candidates $^{3}$, and the transverse momentum of each track is calculated by measuring its helical trajectory inside the magnetic field provided by the solenoid: $p_{T}=B q r$, where $B$ is the magnitude of the magnetic field $(1.4 \mathrm{~T}), q$ is the charge of the particle (in $e$ ), $r$ is the radius of curvature of the track (in $\mathrm{m}$ ) and $p_{T}$ is in $\mathrm{GeV} / c$. The momentum resolution has been measured to be $\sigma_{p_{T}} / p_{T}^{2} \approx 1.7 \times 10^{-3}[\mathrm{GeV} / \mathrm{c}]^{-1}$ using muon cosmic rays.

\subsubsection{Calorimeters}

The primary purpose of the CDF calorimeters is to measure energy and position of charged and neutral particles. The CDF calorimeters instrument two regions with the central barrel calorimeter covering $\left|\eta_{\text {det }}\right|<1$ and the plug calorimeters covering $1.1<$ $\left|\eta_{\text {det }}\right|<3.6$. The calorimeters are segmented into towers that are read out independently. The towers are arranged in such a way that they point toward the center of the CDF detector as shown in Fig. 3.3. Each calorimeter contains sections designed to measure the energy of particles interacting via electromagnetic and hadronic interactions with matter. Each section uses sampling calorimetry with alternating layer of absorbers to favor the development of showers and scintillators to produce photons that are collected to measure the energy deposited in a given tower.

The central region is made of the central electromagnetic (CEM) and hadronic (CHA) calorimeters. The wall hadronic calorimeter (WHA) completes the central coverage of the hadronic section in the region $0.6<\left|\eta_{\text {det }}\right|<1.0$ and provides additional coverage in the forward region out to $\left|\eta_{\text {det }}\right|=1.3$. The central region contains 480 towers each covering 0.1 in $\eta$ and $15^{\circ}$ in $\phi$. Two identical plug calorimeters are present at both ends of the CDF detector. Each contains 480 towers with varying coverage depending on the $\eta_{\text {det }}$ region: for example at $\left|\eta_{\text {det }}\right|=1$, the towers cover 0.1 in $\eta$ and $7.5^{\circ}$ in $\phi$ while at $\eta_{\text {det }}=3.6$ they cover 0.5 in $\eta$ and $15^{\circ}$ in $\phi$. A diagram of the plug calorimeter is shown

\footnotetext{
${ }^{3}$ This procedure is described in Sec. 4.2 .1 .
} 


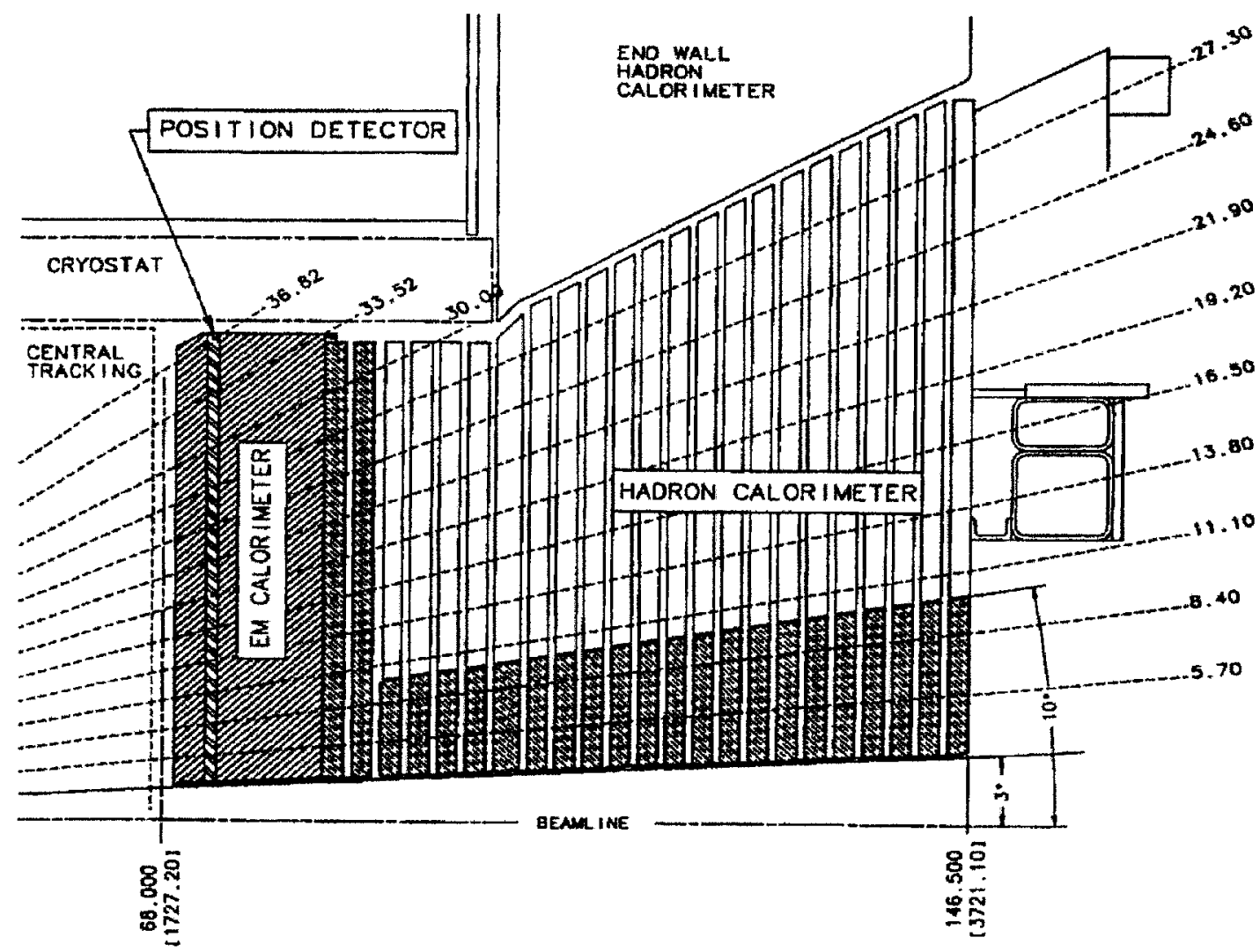

Figure 3.6: Cross section of the upper part of the plug calorimeter.

in Fig. 3.6.

Sampling calorimetry is realized in the electromagnetic calorimeters by interspersing lead sheets acting as absorber (thickness of 0.6 radiation lengths $\left(X_{0}\right)$ for the CEM and $0.8 X_{0}$ for the PEM) and about $5 \mathrm{~mm}$ thick layers of scintillators. For hadronic calorimeters, $2.5 \mathrm{~cm}$ (CHA) and $5.1 \mathrm{~cm}$ (WHA and PHA) of iron absorber is interspersed with scintillators (10 mm thick for CHA and WHA and $6 \mathrm{~mm}$ thick for PHA). In total, the CEM and PEM present $19 X_{0}$ and $21 X_{0}$ of material to a particle, respectively. The longitudinal coverage of hadronic showers in terms of the hadronic interaction length $\lambda$ is $5.5 \lambda$ and $8 \lambda$ in the central and plug region, respectively. These longitudinal coverages are adequate: e.g. $95 \%$ of the energy of a shower generated by a $30 \mathrm{GeV}$ electron $(5 \mathrm{GeV}$ 
pion) is contained in $19 X_{0}$ of lead ( $4 \lambda$ of iron).

Wavelength shifters are employed to collect the photons generated in the scintillator and send them to photomultiplier tubes (PMTs) located behind the calorimeters. The electric signal generated by the PMTs is proportional to the number of scintillation photons, which is itself proportional to the energy of the incident particles. Using $\mathrm{Z}$ boson decays to electron pairs, $Z \rightarrow e e$ and test beam data, the CEM and PEM energy resolutions for electrons and photons have been determined to be $13.5 \% / \sqrt{E_{T}} \oplus 1.5 \%$ and $14.4 \% / \sqrt{E} \oplus 0.7 \%$, respectively. The single particle resolution for hadrons is measured in test beam data to be $75 \% / \sqrt{E}$ (CHA), $80 \% / \sqrt{E}$ (WHA), and $80 \% / \sqrt{E} \oplus 5 \%$ (PHA).

Proportional wire chambers (CES) are embedded in the CEM at a radius corresponding to the maximal development of the shower $\left(\approx 6 X_{0}\right)$. The CES is designed to improve the identification of electrons and photons by measuring the shower profile and by comparing the shower location with the extrapolation of an electron track candidate. It determines the position of an electron shower with a resolution of $2 \mathrm{~mm}$. The plug calorimeter also contains a shower maximum detector located at $6 X_{0}$, the PES, that measures the incoming electromagnetic showers in the forward region using scintillator strips.

Presample shower detectors located in front of the electromagnetic calorimeters are used in the central (CPR) and plug region (PPR) to improve photon identification. They are not used in this analysis.

\subsubsection{Muon Detectors}

Muons are identified in CDF by taking advantage of the fact that they are minimum ionizing particles that lose only modest quantities of energy when passing through large amounts of matter. This is done by positioning drift chambers behind the calorimeters and in some cases behind additional shielding. The majority of particles produced in $p \bar{p}$ collisions reaching these detectors are muons with a relatively small contamination from 
hadronic particles.

The Central Muon Detector (CMU) covers the region $\left|\eta_{\text {det }}\right|<0.6$. It is made of 2,304 single wire drift chambers arranged in 4 concentric layers that provide a maximum of four measurement points per incoming muon candidate. The wires are strung parallel to the beamline, thus providing axial position measurements through the determination of the drift time of ionization electrons. There is a total of $5.5 \lambda$ of material in front of the CMU (corresponding mostly to the material in the CEM and the CHA). The muon identification is also performed by the Central Muon Upgrade (CMP) and the Central Muon Extension (CMX) detectors that use similar wire chambers. The CMP covers the region $\left|\eta_{\text {det }}\right|<0.6$ and is positioned behind the CMU and the CMX covers the region $0.6<\left|\eta_{\text {det }}\right|<1.0$. The CMP is made of a total of 1,075 drift cells staggered in four layers while the CMX is made 2,208 drift cells arranged in 8 layers. An additional muon detector, the IMU, covers the region $1.0<\left|\eta_{\text {det }}\right|<1.5$ but it is not fully commissioned and is not used in this analysis.

Steel plates corresponding to $3 \lambda$ of additional material are placed between the CMU and CMP to reduce the hadronic background. No additional shielding is required for the CMX. Indeed, the particles reaching the CMX must traverse the calorimeter at a larger angle than for the CMU/CMP (since the CMX covers the region $0.6<\left|\eta_{\text {det }}\right|<1.0$ ) and thus pass through larger amount of matter. Figure 3.7 shows the number of absorption lengths as a function of pseudorapidity for the three muon detectors. The minimum $p_{T}$ of muons required to reach the CMU, CMP and CMX is $1.4 \mathrm{GeV} / c, 2.2 \mathrm{GeV} / c$, and $1.4 \mathrm{GeV} / c$, respectively.

\subsubsection{Cherenkov Luminosity Counters}

The Cherenkov Luminosity Counters (CLC) are used to measure the luminosity of the $p \bar{p}$ interactions at the CDF interaction point. The CLC is made of modules of gaseous Cherenkov counters located at each end of the CDF detector, covering $3.6<\left|\eta_{\text {det }}\right|<4.6$. 


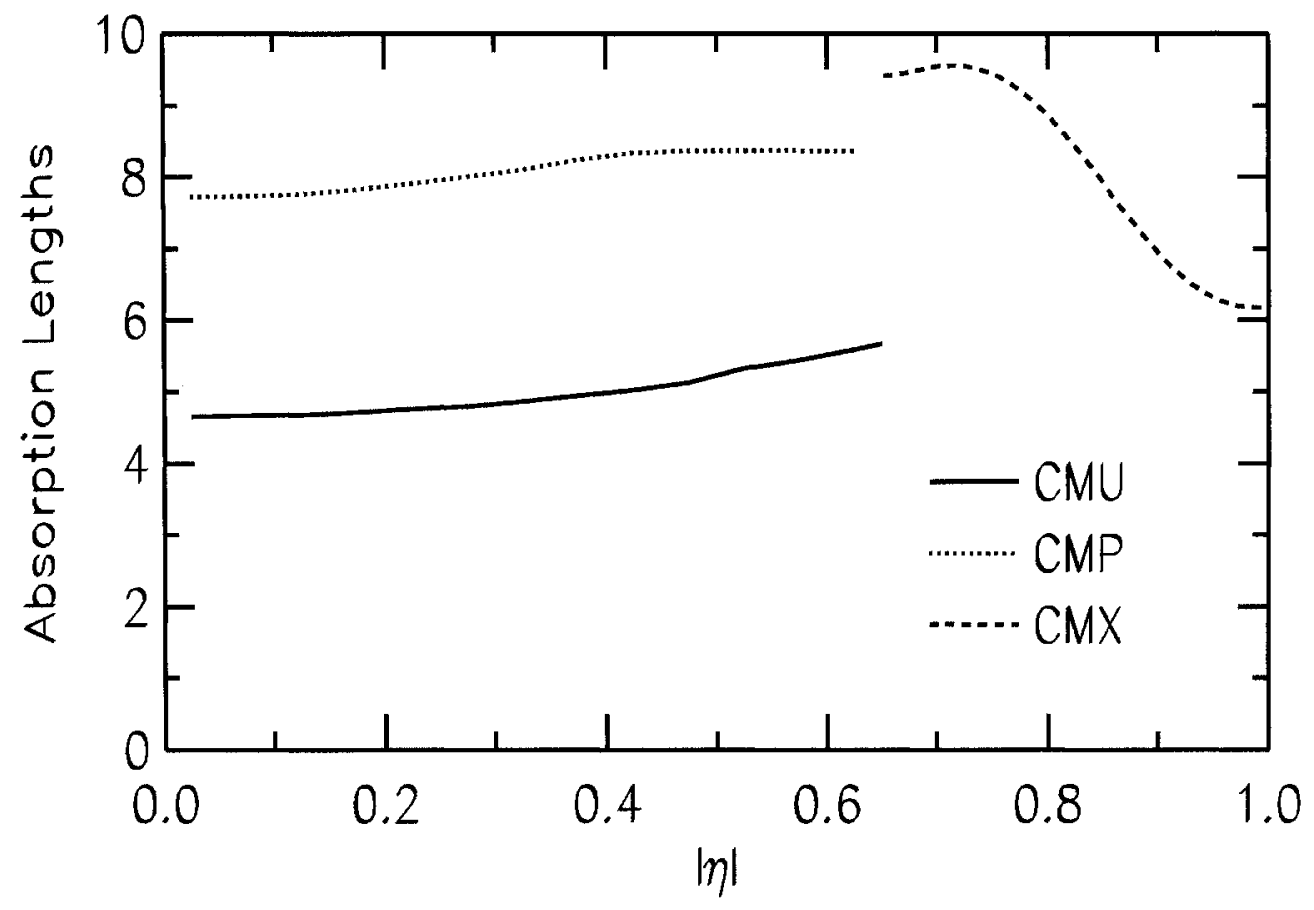

Figure 3.7: Number of absorption lengths as a function of the pseudorapidity for the CMU, CMP and CMX detectors.

The modules carry PMTs that detect the Cherenkov ultraviolet light emitted by the charged particles traversing the modules. Large amount of detected light is indicative of the passage of a number of particles generated from an inelastic collision. With the measurement of the rate of inelastic collisions by the CLC, the instantaneous luminosity of the colliding beam can be extracted using an independent measurement of the inelastic cross-section in $p \bar{p}$ collisions [38] and a measurement of the CLC acceptance.

\subsubsection{Triggers and Data Acquisition System}

The rate of collisions at the Tevatron $(2.5 \mathrm{MHz})$ is too high for each event to be recorded for permanent storage. Fortunately, only a small fraction of the inelastic $p \bar{p}$ collisions produce events of interest. For example, the production of a $Z$ boson or a $t \bar{t}$ pair occur every 25 million and 10 billion inelastic collisions, respectively. It is thus crucial for hadron collider experiments to be equipped with a system capable of analyzing events 
in real time, selecting those worthy of further studies and storing them for subsequent analysis. This is the role played by the CDF trigger and data acquisition system. The trigger system is made of three levels with increasing level of sophistication for event analysis and processing time. The diagram shown in Fig. 3.8 summarizes the system. We describe in this section the trigger and DAQ systems and discuss the available detector information at each trigger level. The specific requirements of the lepton triggers used for this analysis are described in Sec. 4.1.

The Level 1 trigger is made of custom hardware and is optimized for speed. The data for each bunch crossing is recorded and stored in a pipeline where it stays for $6 \mu \mathrm{s}$, the maximum time available for the Level 1 trigger to make its decision to accept or reject the event. Information from various detector systems is combined by the Level 1 trigger. The decision is sent to the front-end detector electronics and if accepted the event data is sent to Level 2 for further analysis.

The calorimeter, COT tracking and muon detectors information is available at Level 1. Jets and electrons are reconstructed as the total and electromagnetic energy deposited in a trigger tower (defined as 2 physical towers adjacent in $\eta_{\text {det }}$ ) above a given threshold. Missing transverse energy and the sum of the energy deposited in the calorimeters are also computed. Evidence for the presence of muons is searched for by looking for aligned hits in the CMU and CMX that are consistent in arrival time. Hits in the CMP are reconstructed based on the hits found in the CMU. Limited charged particle tracking is performed by the eXtremely Fast Tracker (XFT), which examines hits only in axial superlayers of the COT. Track segments are formed in each superlayer that are later linked together to form track candidates. The presence of 10 or 11 hits per track segment (depending on the data-taking period) and the presence of track segments in each four axial superlayers are required. A Track Extrapolation Unit (XTRP) extrapolates the track candidates to the calorimeters and muon detectors to form electron and muon candidates. This information is collected together to make the Level 1 decision. The 
input event rate to the Level 1 trigger is $2.5 \mathrm{MHz}$ (corresponding to the bunch crossing rate) and the maximum output rate is $20 \mathrm{kHz}$, dictated by the Level 2 processing time of about $50 \mu$ s per event.

The data from Level 1 are sent to one of four event buffers that stores them until the Level 2 decision is reached. The Level 2 trigger is made of custom hardware and modified commercial processors. It is made of two main pieces of hardware: the Secondary Vertex Trigger (SVT) and the cluster finder hardware. The SVT uses silicon hit information to trigger on the presence of tracks from displaced vertices. The cluster finder forms cluster of energies from neighboring towers to form more sophisticated electron and jet candidates. The data from these systems as well as the data used for the Level 1 decision are sent to the Level 2 processor crate to make the decision. If accepted, the event data is read from the buffer and sent to the Level 3 trigger. The maximum Level 2 accept rate is $300 \mathrm{~Hz}$.

The Level 3 trigger is made of a farm of 300 commercial dual processors connected by high-bandwidth networks to the digital readout and storage devices. The processors run a version of the offline CDF reconstruction algorithms (described for the objects of interest in Chapter 4) that is optimized for speed. The processing time is about one second per event. Events are accepted by Level 3 at a maximum rate of about $75 \mathrm{~Hz}$. The data for accepted events are sent to permanent storage for subsequent processing by the full offline reconstruction algorithms.

\subsection{Monte Carlo Generation and Detector Simula- tion}

The use of a Monte Carlo (MC) simulation is central to this analysis since the top quark mass is extracted by comparing the kinematics of the candidate events in data with the kinematics of simulated events with different hypotheses for the top quark mass (as 
discussed in detail in Chapter 7). Therefore, the accuracy of this measurement depends directly on the quality of the simulation of the event generation, detector geometry and interactions of particles with the detector material.

The event generation is performed by the HERWIG Monte Carlo generator program [39] for the signal $t \bar{t}$ events. Background events and control samples are generated using HERWIG, PYTHIA [40] and ALPGEN [41] generators. A detailed simulation of the CDF detector [42] based on the GEANT 3 package [43] is used to model the detector's response. Most of the particle interactions with matter are simulated by standard GEANT algorithm except for a few cases. For instance, the charged particle ionization and drift properties in the COT are parametrized and tuned to data. Furthermore, the simulation of showers in the various calorimeters is speeded up by the use of the parametrized shower development package GFLASH [44]. The parameters of the electromagnetic and hadronic showers are tuned to data [45].

The detector response is produced by the detector simulation exactly in the same format as for the real data. Therefore the same reconstruction software discussed in the next Chapter can be applied to both type of events. 


\section{Dataflow of CDF "Deadtimeless" Trigger and DAQ}

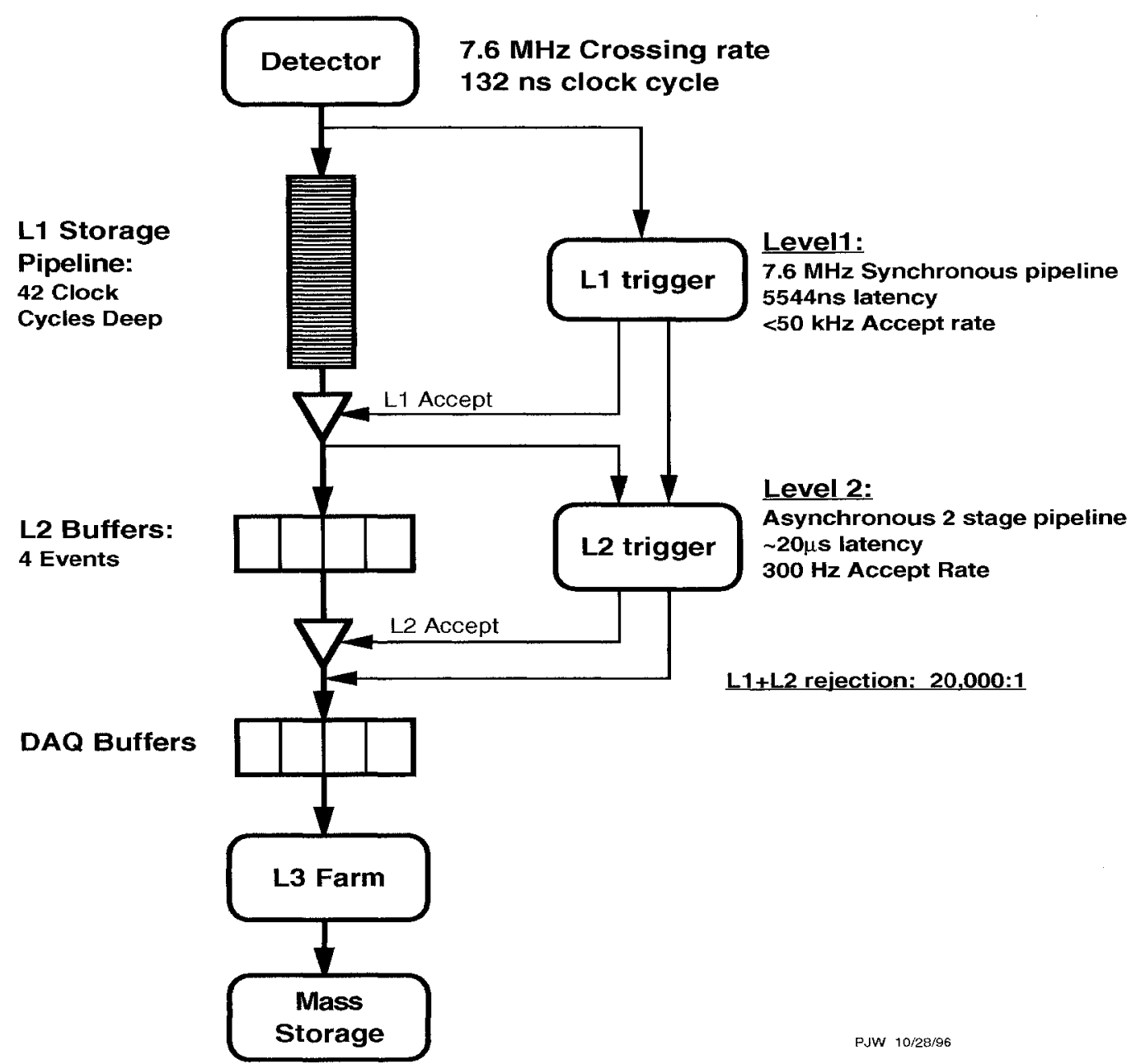

Figure 3.8: Diagram of the data flow in the CDF trigger and data acquisition system. (This picture is from the Technical Design Report [8] dating from 1996, note that the current crossing rate is $2.5 \mathrm{MHz}$, not $7.6 \mathrm{MHz}$, and the clock cycle is $396 \mathrm{~ns}$, not $132 \mathrm{~ns}$.) 


\section{Chapter 4}

\section{Selecting the $t \bar{t}$ Events}

The experimental signature of a lepton+jets $t \bar{t}$ event is as follows: The $t \bar{t}$ pair is produced near threshold as described in Chapter 2 and thus the top quarks tends to be produced centrally with a relatively modest average transverse momentum of $100 \mathrm{GeV} / c$ (compared to their mass of $\approx 175 \mathrm{GeV} / c^{2}$ ). Each top quark decays to a $W$ boson and a $b$-quark. The $b$-jets from the fragmentation of $b$-quarks have an average $p_{T}$ of approximately $75 \mathrm{GeV} / c$. The $W$ bosons decay daughters, i.e. jets from hadronic decays or charged leptons and neutrinos from leptonic decays, have a typical transverse momentum of $50 \mathrm{GeV} / \mathrm{c}$. Each particle from the final state $l \nu q \bar{q}^{\prime} b \bar{b}$ tend to be produced centrally. Indeed the average absolute value of their pseudorapidity is typically $|\eta| \approx 0.7-0.8$. The neutrinos pass through CDF undetected and thus create large apparent missing transverse energy. In this chapter, we present the experimental reconstruction, identification and selection of lepton + jets events, including the requirements applied during data-taking by the trigger system and the final candidate event selection criteria. We also describe the background contamination expected in the data sample. 


\subsection{Trigger Requirements}

The signature of a $t \bar{t}$ lepton+jet event contains many "interesting" characteristics that can be used to identify them over background events: high- $p_{T}$ leptons, large missing transverse energy, large total sum of the transverse energy, heavy flavor jets and high jet multiplicity. At the trigger level, one attempts to select signal events with high efficiency while keeping the trigger accept rate low by rejecting the dominant background events. It is possible to meet these criteria by exploiting only one characteristics of $t \bar{t}$ events: the presence of a centrally produced, high- $p_{T}$ electron or muon.

\subsubsection{Central Electron Trigger}

Electron candidates are selected in the region $\left|\eta_{d e t}\right|<1$ by the Level 1 trigger system by requiring an energy deposit of $8 \mathrm{GeV}$ in a calorimeter trigger tower. The ratio of the energy deposited in the hadronic and electromagnetic sections of the tower $\left(E_{\text {had }} / E_{\text {em }}\right)$ is required to be less than 0.125 to reject hadronic particles. A track with $p_{T}>8 \mathrm{GeV} / c$ found by the XFT is required to point to the tower. At Level 2, a calorimeter cluster is formed by adding adjacent towers with $E_{T}>7.5 \mathrm{GeV}$ to the "seed" tower found at Level 1. The total energy of the cluster is required to have $E_{T}>16 \mathrm{GeV}$ and $E_{\text {had }} / E_{e m}<0.125$. The XFT requirement of Level 1 is confirmed. At Level 3, an electromagnetic cluster (defined in Sec. 4.2.2) with $E_{T}>18 \mathrm{GeV}$ and $E_{h a d} / E_{e m}<0.125$ is required. A fully reconstructed three-dimensional COT track (defined in Sec. 4.2.1) with $p_{T}>9 \mathrm{GeV} / c$ is required to point to the cluster.

The trigger accept rates are $25 \mathrm{~Hz}, 3 \mathrm{~Hz}$ and $1 \mathrm{~Hz}$ at Level 1,2 and 3, respectively, therefore using only a small fraction of the total $75 \mathrm{~Hz}$ bandwidth available at Level 3 . The efficiency of the trigger to select inclusive $W \rightarrow e \nu$ events has been measured to be $96.6 \pm 0.2 \%[46]$ 


\subsubsection{Central Muon Trigger}

Muons are selected using two trigger paths in the region $\left|\eta_{\text {det }}\right|<1$. One path finds muons in the CMU and CMP $\left(\left|\eta_{\text {det }}\right|<0.6\right)$ and the other uses the CMX $\left(0.6<\left|\eta_{\text {det }}\right|<1.0\right)$. The triggers start at Level 1 by finding hits in one or more layers of the CMU or CMX with matching arrival time. For the CMU/CMP trigger, 3 or 4 additional hits in the CMP are required to be consistent with hits in the CMU. An XFT track with $p_{T}$ greater than $4 \mathrm{GeV} / c$ and $8 \mathrm{GeV} / c$ is demanded to match in the $r-\phi$ plane the hits found in the CMU/CMP and CMX, respectively. No additional selections are imposed at Level 2, with the exception that for a fraction of the data-taking period relevant to this analysis, a COT track with $p_{T}>8 \mathrm{GeV} / \mathrm{c}$ was required at Level 2 only for the CMU/CMP trigger. At Level 3, a fully reconstructed three-dimensional COT track with $p_{T}>18 \mathrm{GeV} / \mathrm{c}$ is required to match the stubs (defined in Sec. 4.2.3) reconstructed in the muon chambers.

The trigger accept rates for the CMU/CMP (CMX) trigger paths are $30 \mathrm{~Hz}(2 \mathrm{~Hz})$, $4 \mathrm{~Hz}(2 \mathrm{~Hz})$ and $0.15 \mathrm{~Hz}(0.1 \mathrm{~Hz})$ at Level 1, 2 and 3 , respectively. The combined $\mathrm{CMU} / \mathrm{CMP}$ and CMX trigger efficiency to accept inclusive $W \rightarrow \mu \nu$ events has been measured to be $92.5 \pm 1.1 \%[46]$.

\subsection{Offline Event Selection and Reconstruction}

The events selected by the high- $p_{T}$ lepton triggers are enriched in $W$ and $Z$ boson decays. Further selections must be applied to reduce the large background to $t \bar{t}$ events made primarily of real $W / Z$ bosons in association with high- $E_{T}$ jets and QCD multijets events where one jet fakes the lepton signature. In summary, $t \bar{t} \rightarrow l \nu q \bar{q}^{\prime} b \bar{b}$ events are selected by demanding large missing transverse energy (left by the neutrino) and at least four high- $E_{T}$ jets in the final state coming from the hadronization of quarks. Also, more sophisticated lepton selections than at the trigger level are applied. We describe in this section the event selections that define the lepton+jets $t \bar{t}$ sample. 


\subsubsection{Lepton Track Selection}

The muon or electron coming from the leptonic decay of a $W$ boson is expected to produce a track with high transverse momentum. The main detector used in the reconstruction of these tracks is the COT. The track reconstruction is performed as follows: track segments are formed from hits recorded by the sense wires on each individual COT superlayers. Two separate algorithms are then used to form axial-only tracks: the segment finding algorithm links track segments between axial superlayers while the histogram linking algorithm performs a circle fit that adds axial hits starting from a seed track segment. Tracks found by both algorithms are merged together. Hits in stereo layers are then added using a full three-dimensional fit of the helical trajectory of the track. Finally a fit is performed where the tracks are forced to originate from the beamline in the $x-y$ plane to improve the momentum resolution (known as a beam-constrained fit).

Electron or muon track candidates have the following selections applied: they must have good track segments on three axial and two stereo superlayers. A good track segment is defined as containing at least 5 hits of out the possible 12 in a superlayer. We restrict ourselves to a region with high COT tracking efficiency by requiring the $z$ coordinate of the track trajectory at closest approach to the beam $\left(z_{0}\right)$ to be less than $60 \mathrm{~cm}$ away from the center of the CDF detector.

Additional requirements are applied only to the muon track candidates to remove background events from cosmic rays and decay in flight of pions and kaons. The tracks are required to originate from the center of the detector in the $x-y$ plane and the $\chi^{2}$ over the number of degrees of freedom $\left(\chi^{2} / n d o f\right)$ from the beam-constrained fit is demanded to be less than 2. The cosmic ray background is reduced to negligible level by requiring the track hit timing information to be consistent with the bunch crossing for that event. Finally, the radius at which the track exits the COT is required to be greater than 140 $\mathrm{cm}$ to avoid regions where the muon reconstruction is not well-understood. 


\subsubsection{Electron Selection}

The reconstruction of electron candidates begins by forming electromagnetic clusters in the CEM $\left(\left|\eta_{\text {det }}\right|<1\right)$. The cluster is made of a maximum of two adjacent towers in $\eta$ and the sum of their transverse energy is required to be greater than $20 \mathrm{GeV}$. A COT track with $p_{T}>10 \mathrm{GeV} / c$ is required to point to the most energetic tower of the cluster (seed tower). The COT track is required to be inconsistent with a conversion $\gamma \rightarrow e^{+} e^{-}$. We demand the cluster to have $E_{h a d} / E_{\text {em }}<0.055+0.00045 E$ where $E$ is the total energy of the cluster in $\mathrm{GeV}$ to reject candidates that arise from hadrons faking an electron. The energy dependence of this cut guarantees a high efficiency for real electrons over a large energy range. Background from hadronic particles faking the electron signature are further reduced by requiring the ratio of the cluster transverse energy and track $p_{T}$ $(E / p)$ to be less than two. This cut is applied for clusters with $E_{T}<100 \mathrm{GeV}$ only, because the track momentum measurement becomes increasingly imprecise beyond that. This ratio should be one in principle for real electrons but the cut is larger to account for bremsstrahlung radiation that reduces the track momentum but maintains the same energy deposition in the calorimeter. ${ }^{1}$

A cluster is formed from the energy deposited on the wires of the CES chambers. The position of the CES cluster is required to match the COT track: $-3.0 \mathrm{~cm}<q \times \Delta x<1.5$ $\mathrm{cm}$ (where here $x$ is defined a $r \times \phi$ ) and $|\Delta z|<3.0 \mathrm{~cm}$, where $q$ is the electric charge of the particle. The asymmetry of the $\Delta x$ cut accounts for bremsstrahlung radiation. The lateral profile of the shower as measured in the CEM and CES is required to match profiles of electromagnetic showers measured in test beam data.

Electrons produced in $W$ boson decays are usually isolated (i.e. separated from other neutral and charged particles) with respect to electrons produced in jets. Therefore, we require the $E_{T}$ deposited in the calorimeters in a cone of radius $\Delta R=\sqrt{\Delta \eta^{2}+\Delta \phi^{2}}=$

\footnotetext{
${ }^{1}$ The bremsstrahlung photon is emitted nearly co-linear to a high- $p_{T}$ electron and thus likely to end up in the same calorimeter tower.
} 

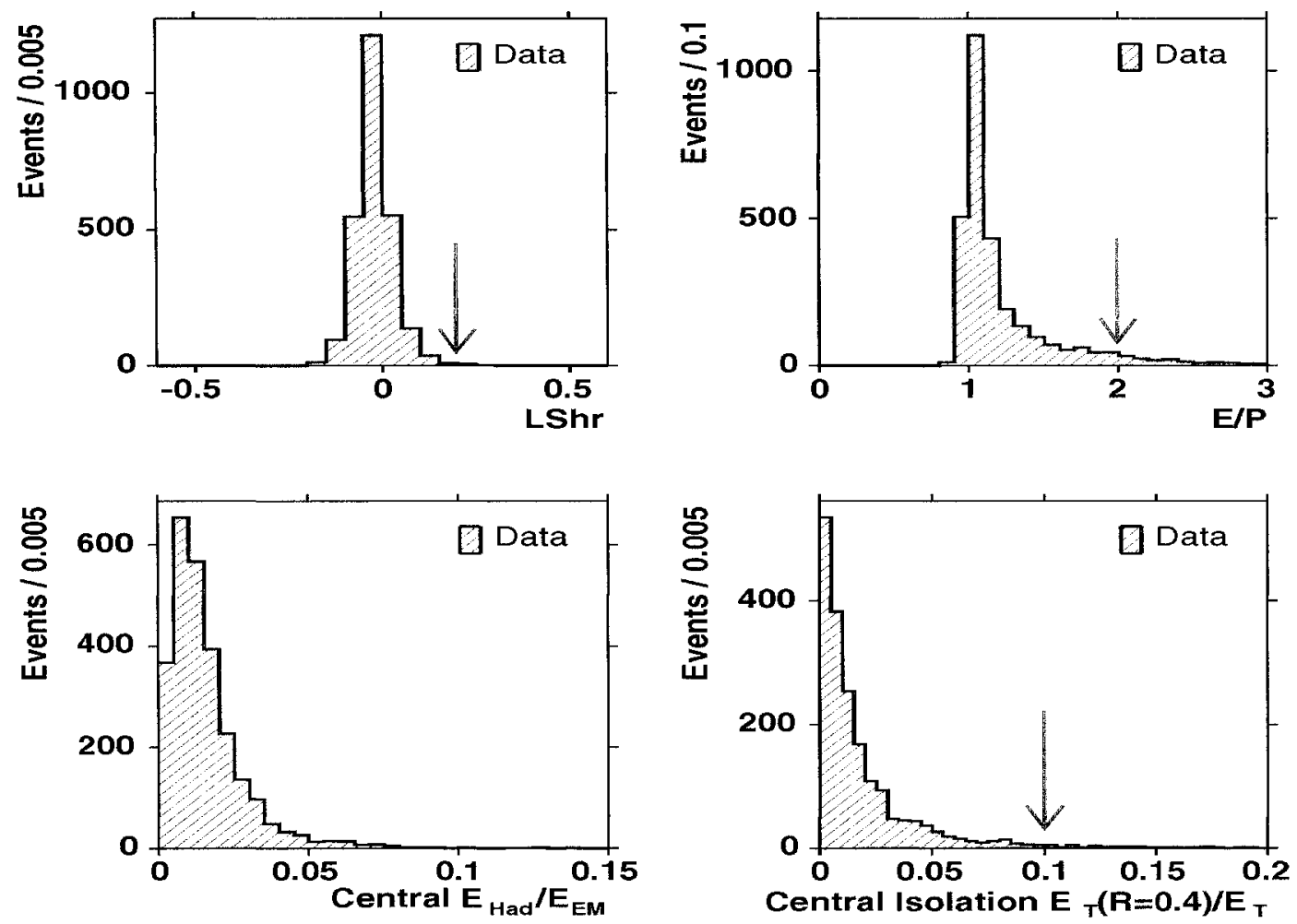

Figure 4.1: Distributions of $L_{s h r}$ (upper left), $E / P$ (upper right), $E_{h a d} / E_{e m}$ (bottom left) and isolation energy (bottom right) for electrons in $Z \rightarrow e e$ data events. $L_{s h r}$ compares the lateral profile of the electron candidate shower in the CEM with the expected profile measured in test beam data. The position of the cuts are indicated by the arrows. There is no single cut requirement for the parameter $E_{h a d} / E_{e m}$

0.4 around the electron candidate, where the electromagnetic cluster towers have been removed, to be less than $10 \%$ of the cluster $E_{T}$.

The distributions of some electron identification variables are shown in Fig. 4.1 for $Z \rightarrow e e$ events that suffer from very little background contamination. The arrows indicate the $t \bar{t}$ lepton selection requirements.

\subsubsection{Muon Selection}

We form muon candidates by matching a COT track with $p_{T}>20 \mathrm{GeV} / c$ to "stubs" in the muon chambers. The stubs are reconstructed as line segments formed by hits in 

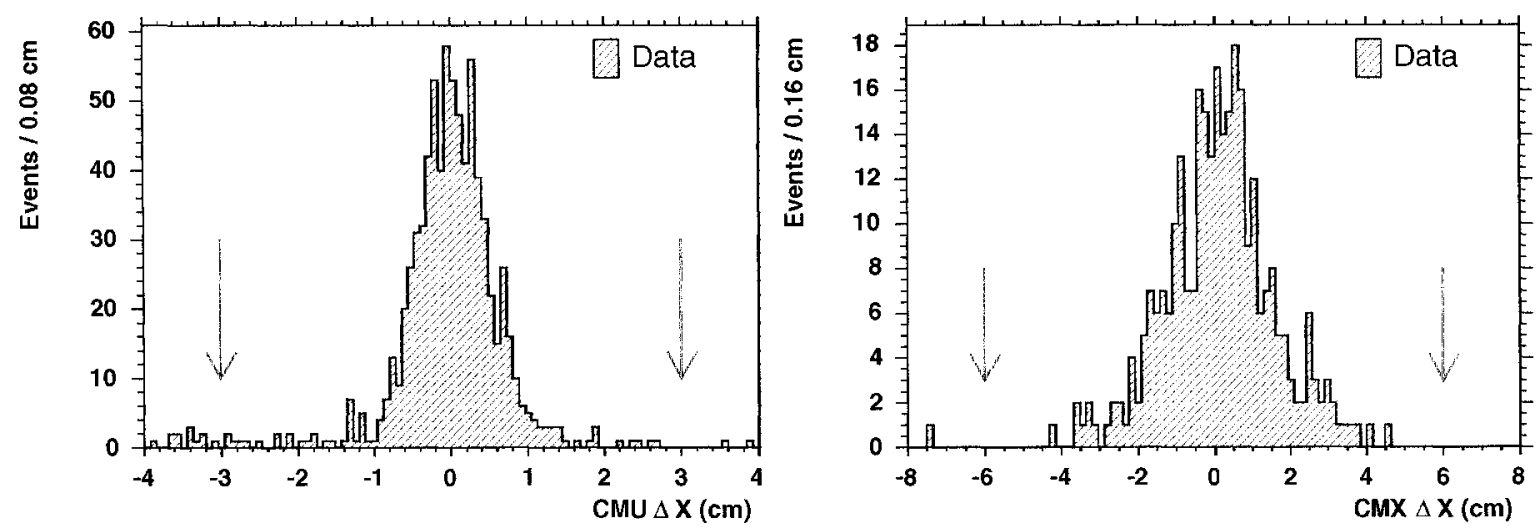

Figure 4.2: Distributions of the distance in the axial direction between the extrapolated track and muon stub positions $(\Delta X)$ in the CMU (left) and CMX (right) detectors in $Z \rightarrow \mu \mu$ data events. The position of the cuts are indicated by the arrows.

the CMU, CMP and CMX. The extrapolated track is required to match the position of the stub in the $r-\phi$ plane within 3,5 and $6 \mathrm{~cm}$ in the CMU, CMP and CMX, respectively. The effect of multiple scattering is taken into account in the size of these cuts. Furthermore, the energy deposited in the calorimeters by the candidate track is required to be consistent with that of minimum ionizing particles to remove fake hadronic particles. Finally, the same isolation requirement as for the electron candidates above is applied, with the exception that the energy in the cone is compared to the track $p_{T}$ instead of the cluster $E_{T}$.

The distributions of some muon identification variables are shown in Fig. 4.2 for $Z \rightarrow \mu \mu$ data events that contain very little background events. The arrows indicate the position of the muon selection requirements.

\subsubsection{Jet Reconstruction}

Jets are groups of particles formed by the hadronization of energetic strongly interacting particles (i.e. gluons or quarks). They are reconstructed as clusters of calorimeter towers using a jet finding algorithm described in this section. We also describe the corrections 
to the jet energy measurement and the algorithm used to identify jets resulting from the hadronization of $b$-quarks.

\section{Jet Clustering Algorithm}

The jet clustering algorithm JETCLU [47] starts with a list of "seed towers", defined as calorimeter towers with $E_{T}>1 \mathrm{GeV}$. If an electron candidate as defined in Sec. 4.2.2 is present in the event, the towers of the corresponding electromagnetic cluster are not used to ensure that the electron candidate is not reconstructed as a jet. Towers inside a cone of radius $\Delta R=\sqrt{\Delta \eta^{2}+\Delta \phi^{2}}=0.4$ around the seed tower are merged to form a proto-jet. The coordinates of the proto-jet centroid are calculated as follows:

$$
\begin{aligned}
\eta^{C} & =\frac{\sum_{i \subset C} E_{T}^{i} \eta^{i}}{E_{T}^{C}} \\
\phi^{C} & =\frac{\sum_{i \subset C} E_{T}^{i} \phi^{i}}{E_{T}^{C}}
\end{aligned}
$$

where the sum is on the tower included in the cone $C, E_{T}^{i}, \eta^{i}$ and $\phi^{i}$ are the variables of the $i$ th tower and $E_{T}^{C}$ is the total $E_{T}$ in the cone. The variables $E_{T}$ and $\eta$ are calculated with respect to the $z$ coordinate of the primary vertex that is calculated from the $z_{0}$ of all good quality tracks in the event. An iterative procedure attempts to match the geometrical center of the cone with the $E_{T}$-weighted centroid coordinates at which point the algorithm stops. The resulting list of stable jets is reduced by applying a splitting and merging procedure that insures that every tower belongs to one and only one jet in an event. The conic shape of the jets is modified by the splitting/merging procedure and final jets can have different shapes. Typical central jets found with a cone of radius $\Delta R=0.4$ are made of about 15 towers on average.

The momentum of an individual tower is a vector with a magnitude equal to the energy deposited in the tower and a direction determined from a unit-vector originating from the interaction point and pointing to the face of the tower. With this definition, 
the energy (momentum) of a jet is simply defined as the sum (vector sum) of the towers that belongs to it. We note that the jets generally have non-equal energy and momentum magnitude that results in a non-zero mass due to the differing angles of the towers that belong to it.

\section{Process-Independent Jet Energy Corrections}

The energy of the jet defined above is not a good estimate of the energy of the parton that initiated the jet fragmentation process. Jet energy corrections are needed to obtain this correspondence. Since the reconstruction of the top quark mass involves the fourmomentum of quarks that make 4 out of 6 final state particles in the lepton+jets channel, the jet energy corrections are central to this analysis. Only the corrections that are process-independent (generic jet corrections) are described here. The discussion of the $t \bar{t}$-specific corrections is postponed until the description of the reconstruction of the top quark mass in Chapter 5.

Different levels of generic jet corrections are applied successively:

1. Relative Correction: The jet energy response varies as a function of the jet pseudorapidity. Indeed, transition regions between the east and west sides of the central calorimeter $\left(\left|\eta_{\text {det }}\right| \lesssim 0.2\right)$ and between the central and plug regions $(0.9 \lesssim$ $\left.\left|\eta_{\text {det }}\right| \lesssim 1.4\right)$ are not as well instrumented as other regions of the calorimeters. Furthermore, the scale of the plug calorimeter response does not correspond exactly to that of the central calorimeter. ${ }^{2}$ The $\eta$-dependence of the jet response is studied using dijet events in which one jet is detected in the central region ("trigger" jet) where the response is well understood. The energy of the second jet ("probe" jet) is studied as a function of its $\eta_{\text {det }}$. Figure 4.3 shows the ratio of the probe and trigger jet $p_{T}$ as a function of $\eta_{d e t}$ of the probe jet for data (black solid points)

\footnotetext{
${ }^{2}$ The calibration technique in both regions are not the same. For example, tracking is not available in the plug region to perform in situ calibrations using charged particles.
} 


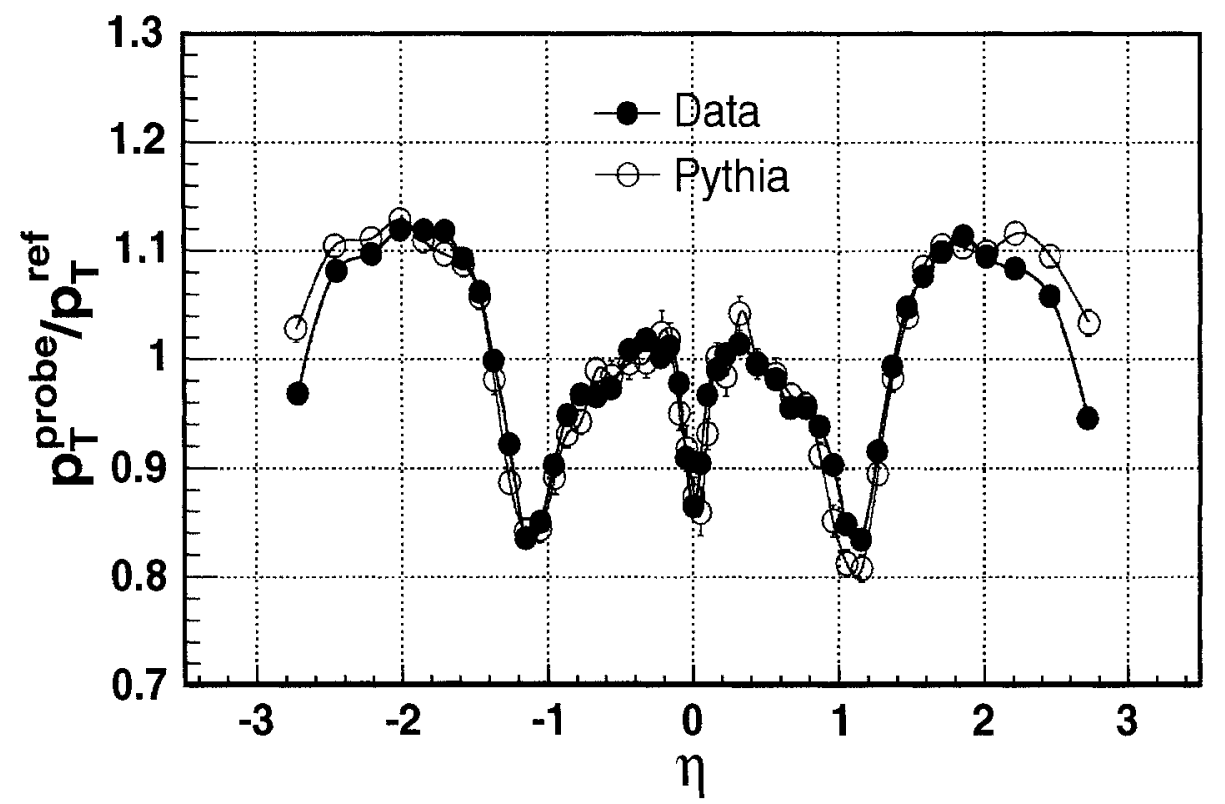

Figure 4.3: Ratio of the $p_{T}$ of the probe and trigger jets as a function of $\eta_{\text {det }}$ of the probe jet in dijet balancing for data (black solid points) and Monte Carlo events (red open points).

and PYTHIA Monte Carlo events (red open points). Good agreement is observed between data and MC up to $\left|\eta_{\text {det }}\right|=2 .^{3}$ Variations of the jet response as large as $20 \%$ are observed as a function of $\eta_{\text {det }}$. A correction is extracted from this plot to make the jet energy response uniform for all $\eta_{\text {det }}$.

2. Multiple Interactions Correction: There can be more than one inelastic collision per bunch crossing at the Tevatron. Additional interactions simultaneous with the primary interaction can deposit energy inside the jet cone and therefore bias its energy measurement. A correction that subtracts the average energy deposited in a jet cone is extracted in data as a function of the number of additional interactions. The correction is small: it is measured to be $0.36 \mathrm{GeV}$ per jet per additional interaction, corresponding to less than $1 \%$ of a typical jet energy in $t \bar{t}$ events.

\footnotetext{
${ }^{3}$ The discrepancy for $\left|\eta_{\text {det }}\right|>2$ does not affect our analysis since we select jets in the region $\left|\eta_{\text {det }}\right|<2$ as discussed later.
} 
3. Absolute Energy Corrections: Quarks and gluons fragment into a large variety of particle species that interact differently with the calorimeter material. For instance electrons and photons interact electromagnetically and the measured energy in the calorimeter increases linearly with the input particle energy. The behavior of hadronically interacting particles (like a $\pi^{ \pm}$) is more complicated. For instance, the detected energy of single charged pions has been observed to vary non-linearly as a function of input particle $p_{T}$. Since it is impossible to identify single particles resulting from the fragmentation process on a jet-by-jet basis, the measured energy of a jet is corrected using an average configuration of the particles species and momentum spectrum inside a jet. Such corrected energy corresponds to the true hadron-level energy deposited inside a jet on average. The correction is extracted from dijet Monte Carlo events assuming a flat $p_{T}$ spectrum ${ }^{4}$. The accuracy of the correction depends on the realistic simulation of two aspects of the jet process: (1) the fragmentation process and (2) the single particle response for the different particle species. Uncertainties on these two aspects affect significantly the top quark mass measurement as discussed in Sec. 5.4. The resulting correction is shown in Fig. 4.4 as a function of the jet $p_{T}$. The correction factor is about 1.35 for $p_{T}$ of 20 $\mathrm{GeV}$ and reduces to about 1.1 at large jet $p_{T}$. The uncertainty on the corrections is about $2 \%$ at low $p_{T}$ and rises to about $3 \%$ at large $p_{T}$.

\section{$B$-Jet Tagging}

The identification of $b$-jets is important to improve the reconstruction of the top quark mass and reduce the background contamination. The tagging of $b$-jets is not imposed as a requirement in this analysis, but is used to separate the $t \bar{t}$ sample into event categories as described in Sec. 4.3.

\footnotetext{
${ }^{4} \mathrm{~A}$ flat $p_{T}$ spectrum is used to make corrections as independent as possible of a specific physics process.
} 


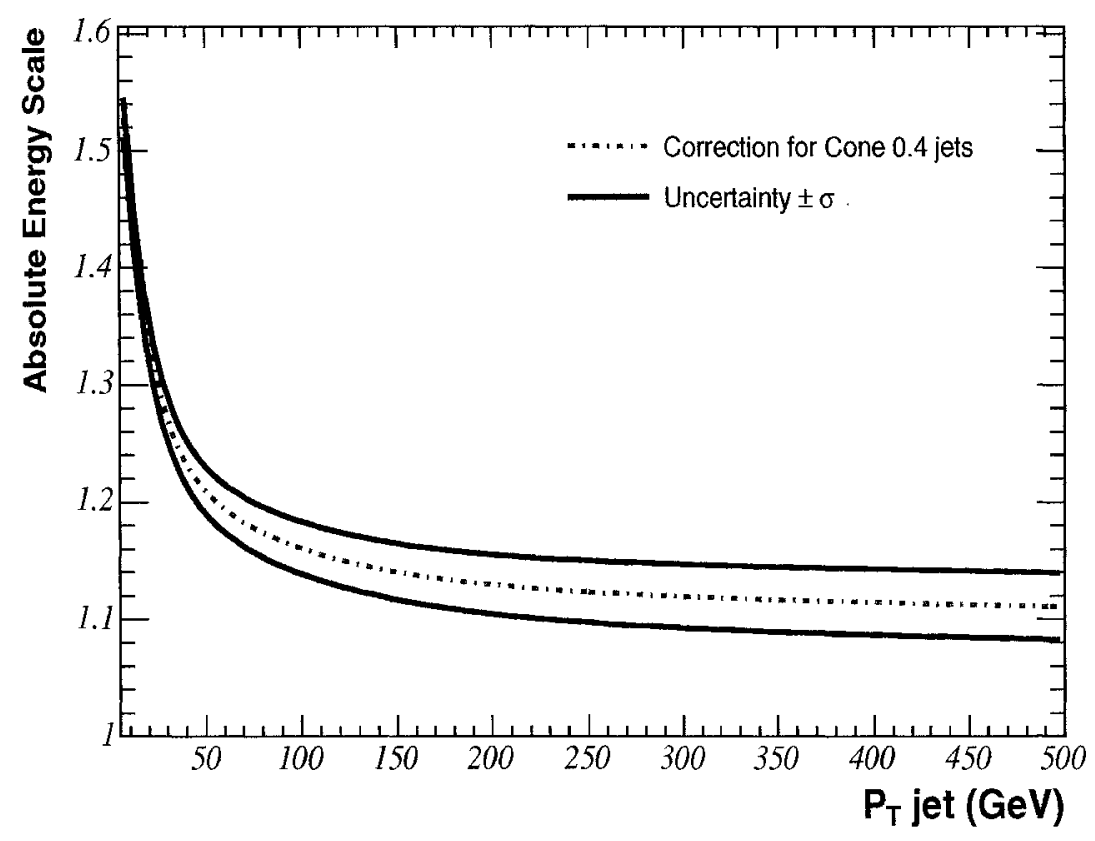

Figure 4.4: Absolute energy scale correction as a function of the jet $p_{T}$. The uncertainty (dominated by the hadronic particle response) is also shown on the plot.

The identification of $b$-jets uses the fact that $b$-hadrons have a longer lifetime than light quark hadrons on average. Since $b$-quarks from top quark decays are highly boosted, they travel a sufficient distance such that the secondary vertex reconstructed from the $b$-hadron decay products can be distinguished from the primary vertex using the very good position resolution of the silicon detectors. We use the SECVTX [21] algorithm that considers jets with uncorrected $E_{T}>15 \mathrm{GeV}$. The algorithm looks inside the jets for good quality tracks containing both COT and silicon information that form a secondary vertex. The distance in the $r-\phi$ plane between the primary and secondary vertex $\left(L_{x y}\right)$ and its uncertainty $\left(\sigma\left(L_{x y}\right)\right)$ are calculated. If the secondary vertex in the jet is significantly displaced, i.e. if $L_{x y} / \sigma\left(L_{x y}\right)>7.5$, the jet is considered as tagged. The $b$ tagging efficiency ${ }^{5}$ per jet is shown in Fig. 4.5 as a function of the jet $E_{T}$. The efficiency

\footnotetext{
${ }^{5}$ The efficiency is defined as the number of tagged jets over the total number of jets that are succesfully matched to a b-quark.
} 


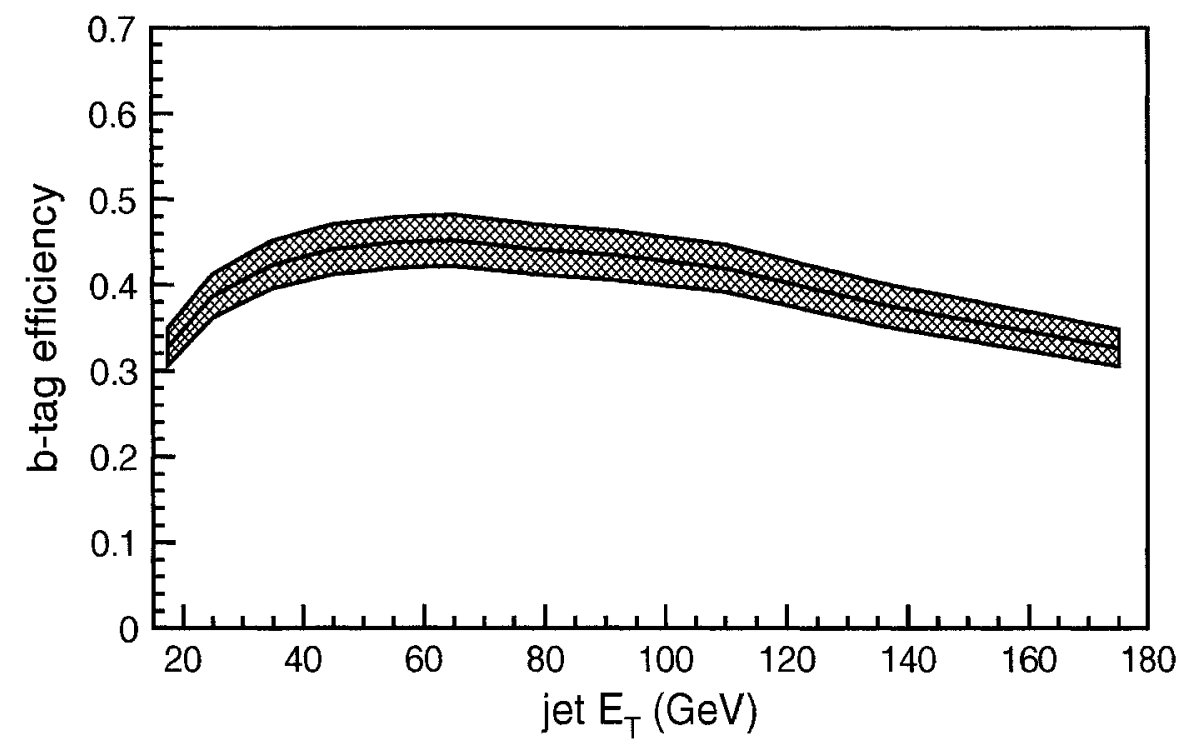

Figure 4.5: SECVTX $b$-tagging efficiency as a function of the uncorrected jet $p_{T}$ in the region $\left|\eta_{\text {det }}\right|<1$. The shaded region shows the uncertainty on the measured efficiency.

is about $35 \%$ for jet $E_{T}$ of $25 \mathrm{GeV}$ and rises up to $45 \%$ for jet $E_{T}$ of $60 \mathrm{GeV}{ }^{6}$ The efficiency to tag at least one $b$-jet in a $t \bar{t}$ events is about $60 \%$. The algorithm erroneously tags light quark jets ("mistags") at a rate of about $1 \%$ per jet.

\section{Jet Selection}

We require at least 3 jets with $E_{T}>15 \mathrm{GeV}$ and at least one with $E_{T}>8 \mathrm{GeV}$ per event, in both cases in the region $\left|\eta_{\text {det }}\right|<2.0$, where $E_{T}$ is corrected for the relative and multiple interactions corrections. This is the minimum number of jets to reconstruct the top quark mass since two jets should come from the hadronic decay of a $W$ boson and two jets should result from the fragmentation of the two $b$-quarks in the lepton+jets final state. The jet $E_{T}$ thresholds and the required number of $b$-tags depend of the subsample to which the event belongs, and is discussed in Sec. 4.3.

\footnotetext{
${ }^{6}$ The efficiency decreases for jet $E_{T}$ beyond $60 \mathrm{GeV}$ because fewer good quality tracks are found as the track density increases inside the jets.
} 


\subsubsection{Missing Transverse Energy}

By energy-momentum conservation, the total transverse energy flow in an event should be equal to zero (neglecting the measurement uncertainties). The $z$ component of the total momentum in the event cannot be used as a constraint in hadron colliders since we are unaware of the momentum of the initial state partons inside the colliding protons and antiprotons. The neutrino from the $W$ boson decay traverses through the CDF detector undetected and thus produces a large apparent imbalance in the total transverse energy flow as measured by the calorimeters. The missing transverse energy $\mathscr{E}_{T}$ is defined as:

$$
E_{T}=\left|-\sum_{i} \overrightarrow{E_{T}^{i}}\right|
$$

where the sum is over all the towers in the calorimeters and $\overrightarrow{E_{T}^{i}}$ is a vector originating from the primary vertex interaction pointing to the face of the tower and with norm equal to the $E_{T}$ measured in the tower. When a muon candidate (as defined in Sec. 4.2.3) is present in the event, the muon track $p_{T}$ is used in the $\mathscr{E}_{T}$ reconstruction instead of the $E_{T}$ deposited by the muon in the calorimeters. The energy of towers belonging to jets are corrected by the jet energy corrections for the relative and multiple interactions correction in the $E_{T}$ reconstruction. We demand the $E_{T}$ in the event to be greater than $20 \mathrm{GeV}$.

\subsubsection{Final Event Selection}

Backgrounds from $Z / \gamma$ Drell-Yan events and $t \bar{t}$ dilepton events are reduced by requiring the presence of one and only one identified lepton as defined previously. We also veto events in which an additional electron is identified in the forward region [22]. The minimum $\chi^{2}\left(\chi_{m i n}^{2}\right)$ returned by the kinematic fit based on the hypothesis that the event is $t \bar{t}$ is required to be less than 9 (the $\chi^{2}$ kinematic fit is described in detail in Chapter 5 ). This selection is only applied for the reconstruction of the top quark mass. The motivation for omitting this cut for the reconstruction of the hadronic $W$ boson mass $W \rightarrow j j$ is 
described in more detail in Chapter 6 . This difference in selection results in two distinct samples: the top quark mass sample (with $\chi_{\min }^{2}<9$ ) and the $W$ boson mass sample (no $\chi_{m i n}^{2}$ cut), also referred in this document as the $m_{t}^{r e c o}$ and $m_{j j}$ samples, respectively.

The total efficiency to select $t \bar{t}$ events is approximately $5 \%$ using the event selections described in this chapter (with four jets with $E_{T}>15 \mathrm{GeV}$ and no $\chi_{\min }^{2}$ cut). This efficiency drops to $3 \%$ and $1 \%$ when requiring the presence of at least one and two $b$-tags, respectively.

Finally, the dataset used in this analysis is composed of events in which every detector component described in Chapter 3 was functioning well during data-taking. The final data sample is made of an integrated luminosity of $318 \mathrm{pb}^{-1}$ and contains 138 and 165 events, respectively, in the $m_{t}^{r e c o}$ and $m_{j j}$ samples that only differs by the $\chi_{\text {min }}^{2}$ cut as described above. We further divide this dataset into four subsamples as described next.

\subsection{Division of the Lepton+Jets Sample}

Better statistical performance in the top quark mass measurement can be achieved by fitting separately events with different sensitivity to the top quark mass. The sensitivity depends primarily on the reconstructed top quark mass resolution and the background contamination. These two factors are in turn dependent on the number of jets with a $b$ tag and the jet $E_{T}$ threshold, on which basis we separate both the $m_{t}^{\text {reco }}$ and $m_{j j}$ samples into four non-overlapping categories:

- 2-tag events: 2 -tags and $\geq 3$ jets with $E_{T}>15 \mathrm{GeV}$ and a jet with $E_{T}>8 \mathrm{GeV}$;

- $1-\operatorname{tag}(\mathrm{T}): 1 b$-tag and $\geq 4$ jets with $E_{T}>15 \mathrm{GeV}$;

- $1-\operatorname{tag}(\mathrm{L}): 1 b$-tag, 3 jets with $E_{T}>15 \mathrm{GeV}$ and $\geq 1$ jet with $8 \mathrm{GeV}<E_{T}<15$ $\mathrm{GeV}$; and

- 0-tag: no $b$-tag and $\geq 4$ jets with $E_{T}>21 \mathrm{GeV}$. 
The SECVTX tags have to be on one of the four highest $E_{T}$ jets to be counted in the categories above. The relative and multiple interactions corrections described in Sec. 4.2 .4 have been applied before applying the jet $E_{T}$ cuts. The jet $E_{T}$ threshold for 0 -tag events is increased with respect to other subsamples to further suppress the background that is larger for this subsample.

The reconstructed top quark and $W$ boson mass resolutions are affected by the combinatorial ambiguity of assigning jets in the final state to the corresponding quark. Since $b$-tagged jets are automatically assigned to $b$-quarks in the $\chi^{2}$ kinematic fitter (as described in Chapter 5), the probability to pick the correct jet-to-parton assignment improves with the number of $b$-tags in the event. This is illustrated by Fig. 4.6 showing the reconstructed top quark mass (that is the subject of Chapter 5) for events with $M_{t o p}=$ $178 \mathrm{GeV} / c^{2}$ for each subsample. As can be seen in these plots, the fraction of correct jet-parton assignments (illustrated by the inner blue histogram) increases from $20 \%$ for 0 -tag events to $47 \%$ for 2 -tag events. Consequently, the resolution of the 0 -tag mass distribution is about $15 \%$ and $25 \%$ worse than for 1 -tag and 2 -tag events, respectively.

The top quark mass measurement improves also with lower background contamination. This is influenced by $b$-tagging given that $t \bar{t}$ events contain at least two $b$-jets while only a few percent of the dominant $W+$ jets background events contain heavy flavor jets [21]. Indeed the expected signal over background ratio $(\mathrm{S} / \mathrm{B}))$ is 2.2 and 10.6 for 1-tag and 2-tag events, respectively. It depends also on the jet $E_{T}$ threshold because $t \bar{t}$ events produce more energetic jets than background events on average. For instance, the S/B ratio is 1.1 for $1-\operatorname{tag}(\mathrm{L})$ events and 3.7 for $1-\operatorname{tag}(\mathrm{T})$ events, which motivates the division of the 1-tag sample.

The requirements and expected signal to background ratio for each subsample are summarized in Table 4.1. There is no a priori background estimate available for the 0 -tag subsample. Also given in Table 4.1 is the distribution in each event categories of the 138 and 165 events in the top quark and $W$ boson mass samples, respectively. 

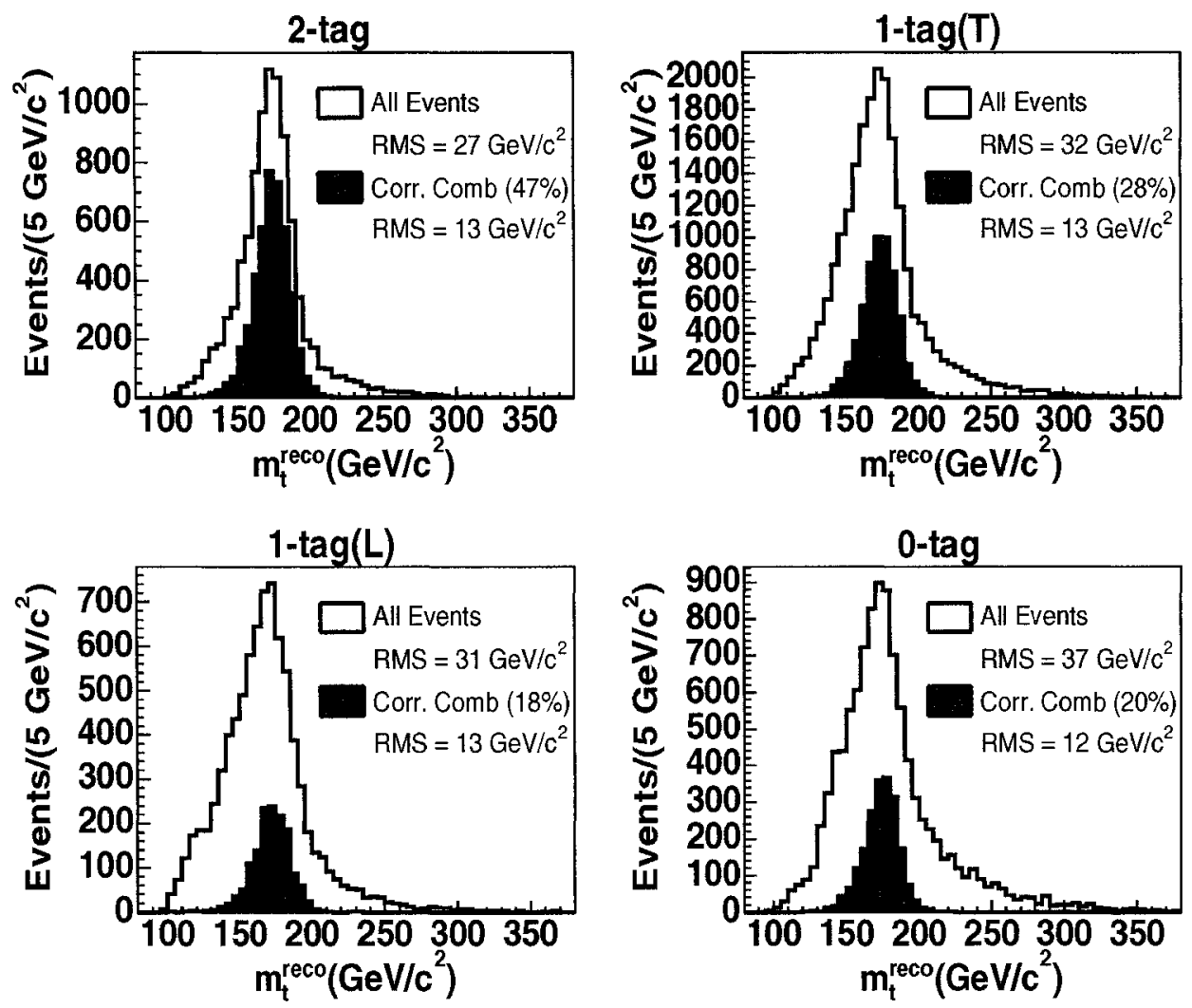

Figure 4.6: Reconstructed top quark mass $\left(m_{t}^{r e c o}\right)$ for 2-tag (upper left), 1-tag(T) (upper right), 1-tag(L) (bottom left) and 0 -tag (bottom right) for $t \bar{t}$ events with $M_{\text {top }}=178$ $\mathrm{GeV} / \mathrm{c}^{2}$. The yellow (outer) histograms show the mass distributions for all events and the blue (inner) histograms show the distributions only for the case where the $\chi^{2}$ fitter picks the correct jet-parton assignment.

\subsection{Background Contamination}

We estimate the sources of background in the lepton+jets $t \bar{t}$ sample separately for each subsample since the size of the background contamination and its nature varies with the number of $b$-tagged jets and jet $E_{T}$ thresholds. The number of background events expected in each $b$-tagged subsample is used in the likelihood fit used to extract the top quark mass as described in Chapter 7. We discriminate signal and background in the 0-tag subsample only using the different signal and background mass shapes. 


\begin{tabular}{|c|c|c|c|c|}
\hline Category & $2-\operatorname{tag}$ & $1-\operatorname{tag}(\mathrm{T})$ & $1-\operatorname{tag}(\mathrm{L})$ & 0 -tag \\
\hline Jet $E_{T} \quad \mathrm{j} 1-\mathrm{j} 3$ & $E_{T}>15$ & $E_{T}>15$ & $E_{T}>15$ & $E_{T}>21$ \\
\hline $\operatorname{cuts}[\mathrm{GeV}] \quad \mathrm{j} 4$ & $E_{T}>8$ & $E_{T}>15$ & $E_{T}>8$ & $E_{T}>21$ \\
\hline$b$-tagging & 2 tags & $1 \mathrm{tag}$ & $1 \mathrm{tag}$ & 0 tag \\
\hline Expected S/B & 10.6:1 & $3.7: 1$ & $1.1: 1$ & $\mathrm{~N} / \mathrm{A}$ \\
\hline $\begin{array}{c}\text { Number of events } \\
\text { no } \chi_{\min }^{2} \text { cut }\end{array}$ & 25 & 63 & 33 & 44 \\
\hline $\begin{array}{l}\text { Number of events } \\
\text { with } \chi_{\min }^{2}<9\end{array}$ & 16 & 57 & 25 & 40 \\
\hline
\end{tabular}

Table 4.1: Jet $E_{T}$ cut and $b$-tagging requirement for the four event categories. Also shown is the expected signal to background ratio (S/B) for each subsample as well as the number of events observed in data before and after the $\chi_{\min }^{2}$ cut.

The dominant sources of background are:

- $W$ theavy flavor: $W$ boson events produced in association with heavy flavor jets: $W b \bar{b}, W c \bar{c}, W c$.

- $W+$ jets mistag: $W$ boson events produced in association with light flavor jets. One of the jets is falsely identified as a $b$-jet by SECVTX.

- QCD events: Multijets events produced via QCD in which one of the jets fake a lepton. The $\mathscr{E}_{T}$ is falsely created by a mis-measurement of the jets transverse energy.

- Other electroweak processes: Dibosons ( $W W, W Z$ and $Z Z$ ) and single-top production.

The expected contribution from each of these sources is calculated using data events for $W+$ jet and QCD events and Monte Carlo events for the dibosons and single-top background sources. A detailed description of the techniques used to estimate the background 


\begin{tabular}{lccc}
\hline \hline Source & \multicolumn{3}{c}{ Background estimate $\left(m_{j j}\right.$ sample $)$} \\
& 2 -tag & $1-\operatorname{tag}(\mathrm{T})$ & $1-\operatorname{tag}(\mathrm{L})$ \\
\hline$W+$ heavy flavor & $1.12 \pm 0.43$ & $3.91 \pm 1.23$ & $6.81 \pm 1.85$ \\
$W+$ jets mistags & $0.40 \pm 0.08$ & $3.22 \pm 0.41$ & $4.14 \pm 0.53$ \\
QCD multijets & $0.31 \pm 0.08$ & $2.32 \pm 0.50$ & $2.04 \pm 0.53$ \\
Diboson & $0.05 \pm 0.01$ & $0.45 \pm 0.10$ & $0.71 \pm 0.13$ \\
single-top & $0.01 \pm 0.00$ & $0.49 \pm 0.09$ & $0.60 \pm 0.11$ \\
\hline Total & $1.9 \pm 0.5$ & $10.4 \pm 1.7$ & $14.3 \pm 2.5$ \\
\hline \hline
\end{tabular}

Table 4.2: Background event estimates for the $W$ boson mass sample for $\int \mathcal{L} d t=318$ $\mathrm{pb}^{-1}$ (in number of expected events).

in the $t \bar{t}$ sample is provided in [21]. The result of the calculation is given in Table 4.2 for the $W$ boson mass sample (no $\chi_{\min }^{2}$ cut).

The efficiency of the $\chi_{\min }^{2}$ cut, that is discussed in more details in Sec. 5.3, needs to be calculated for each background source in order to estimate the background in the top quark mass sample. This is done using Monte Carlo simulation. The $\chi_{\min }^{2}$ cut efficiencies are similar for all background sources and range from about $35 \%$ for 2 -tag events to about $75 \%$ for 1-tag events. The background estimate for the top quark mass sample is given in Table 4.3 . 


\begin{tabular}{lccc}
\hline \hline Source & \multicolumn{3}{c}{ Background estimate $\left(m_{t}^{\text {reco }}\right.$ sample $)$} \\
& 2-tag & $1-\operatorname{tag}(\mathrm{T})$ & $1-\operatorname{tag}(\mathrm{L})$ \\
\hline$W+$ heavy flavor & $0.37 \pm 0.14$ & $2.70 \pm 0.85$ & $4.56 \pm 1.24$ \\
$W+$ jets mistags & $0.18 \pm 0.04$ & $2.48 \pm 0.32$ & $3.19 \pm 0.41$ \\
QCD multijets & $0.14 \pm 0.04$ & $1.79 \pm 0.39$ & $1.57 \pm 0.42$ \\
Diboson & $0.02 \pm 0.00$ & $0.31 \pm 0.07$ & $0.47 \pm 0.09$ \\
single-top & $0.00 \pm 0.01$ & $0.36 \pm 0.07$ & $0.40 \pm 0.07$ \\
\hline Total & $0.7 \pm 0.2$ & $7.6 \pm 1.2$ & $10.2 \pm 1.7$ \\
\hline \hline
\end{tabular}

Table 4.3: Background event estimates for the top quark mass sample for $\int \mathcal{L} d t=318$ $\mathrm{pb}^{-1}$ (in number of expected events). 


\section{Chapter 5}

\section{Top Quark Mass Reconstruction}

The top quark mass is extracted by comparing the distribution of a kinematic variable reconstructed in the data with MC templates created for various $M_{t o p}$ hypotheses. The statistical precision of the measurement is therefore strongly dependent on the sensitivity of the kinematical quantity in question to $M_{t o p}$. In this analysis, we use the reconstructed top quark mass $m_{t}^{r e c a}$ as determined by a $\chi^{2}$ kinematic fit that has been used in previous measurements $[10,16]$. We emphasize that the reconstructed top quark mass $m_{t}^{\text {reco }}$ is not equivalent to the true top quark mass $M_{\text {top }}$, and we correct for the difference statistically using the template technique.

The variable $m_{t}^{\text {reco }}$ is chosen for its sensitivity to $M_{t o p}$. However, $m_{t}^{\text {reco }}$ is also sensitive to the jet energy scale, which introduces a large systematic uncertainty in the measurement. The uncertainty on JES can be reduced by the use of another kinematic variable, the hadronic $W$ boson mass $m_{j j}$, that is also sensitive to JES while being mostly independent of $M_{t o p}$. The reconstruction of $m_{j j}$ is the subject of the next Chapter.

\subsection{The $\chi^{2}$ Kinematic Fit}

The variable $m_{t}^{\text {reco }}$ is obtained by minimizing the following $\chi^{2}$ expression that is based on the hypothesis that the event of study arises from $t \bar{t}$ production and decay: 


$$
\begin{aligned}
\chi^{2} & =\Sigma_{i=\ell, 4 j e t s} \frac{\left(\hat{p}_{T}^{i}-p_{T}^{i}\right)^{2}}{\sigma_{i}^{2}}+\Sigma_{j=x, y} \frac{\left(\hat{p}_{j}^{U E}-p_{j}^{U E}\right)^{2}}{\sigma_{j}^{2}} \\
& +\frac{\left(m_{q q^{\prime}}-m_{W}\right)^{2}}{\Gamma_{W}^{2}}+\frac{\left(m_{\ell \nu}-m_{W}\right)^{2}}{\Gamma_{W}^{2}}+\frac{\left(m_{b q q^{\prime}}-m_{t}^{r e c o}\right)^{2}}{\Gamma_{t}^{2}}+\frac{\left(m_{b \ell \nu}-m_{t}^{r e c o}\right)^{2}}{\Gamma_{t}^{2}}
\end{aligned}
$$

The first term constrains the fitted transverse momentum of the lepton and four highest $E_{T}$ jets, $\hat{p}_{T}^{i}$, to the measured value $p_{T}^{i}$ within the experimental resolutions $\sigma_{i}$. The second term constrains the fitted $x$ and $y$ components of the unclustered energy $\hat{p}_{j}^{U E}$ to the measured value $p_{j}^{U E}$ within their experimental resolutions $\sigma_{j}$. The unclustered energy is defined as all the energy in the event that is not due to the lepton and the four highest $E_{T}$ jets and it is used to infer the $p_{T}$ of the neutrino. The precise calculation of the momentum and energy of the lepton, jets and unclustered energy as well as their experimental resolution is the subject of the next section.

The $\chi^{2}$ expression also contains terms that constrain the reconstructed hadronic and leptonic $W$ boson masses $\left(m_{q q^{\prime}}\right.$ and $\left.m_{\ell \nu}\right)$ to the world average $m_{W}=80.42 \mathrm{GeV} / c^{2}[5]$ with an uncertainty corresponding to the $W$ boson intrinsic width $\Gamma_{W}=2.12 \mathrm{GeV}$ [5]. Furthermore, the reconstructed leptonic and hadronic top quark masses $\left(m_{b q q^{\prime}}\right.$ and $\left.m_{b \ell \nu}\right)$ are required to be equal within the top quark intrinsic width $\Gamma_{t}$, which is set to $1.5 \mathrm{GeV} / c^{2}$

We note that the fit consists in minimizing a two-constraint $\chi^{2}$. On one hand, six independent constraints are employed: the two reconstructed $W$ boson masses must equal the known value, each of the reconstructed top quark mass must equal $m_{t}^{r e c o}$ and the sum of each component of the transverse momentum of the event must be zero. On the other hand, there are four unmeasured quantities: the three-momentum of the neutrino and the top quark mass $m_{t}^{\text {reco }}$.

The reconstruction of the top quark mass necessitates the knowledge of the correct jet-parton assignment. Since this is not known a priori, the $\chi^{2}$ is minimized for all possible jet-parton assignments made from the four highest $E_{T}$ jets for each event. This results in 12 reconstructed top quark masses per event (since the two $W$ daughter jets 
are interchangeable in the reconstruction). In addition, the $z$ component of the neutrino momentum $p_{z}^{\nu}$ cannot be measured directly and its initial value is inferred from the missing $E_{T}$ and lepton momentum measurements and by assuming that the reconstructed leptonic $W$ boson mass equals $m_{W}=80.42 \mathrm{GeV} / c^{2}$. This results in a quadratic equation in $p_{z}^{\nu}$ and thus two solutions exist in general. These two solutions are attempted and increase the number of possible reconstructed masses to 24 per event. The reconstructed top quark mass used for further analysis is the one coming from the combination that yields the lowest value of the $\chi^{2}\left(\chi_{\min }^{2}\right)$.

The number of combinations is reduced by assigning jets with a $b$-tag only to $b$-quarks in the $\chi^{2}$ fit. Consequently, events with 0-, 1- and 2-tag have 24, 12 and 4 combinations, respectively.

\subsection{Description of the Input to the $\chi^{2}$ Fit}

The 4-momentum of the lepton, jets and unclustered energy are used as input to the $\chi^{2}$ fit as well as their uncertainties as described above. Therefore, providing the best estimate and the most accurate experimental uncertainty of these variables will improve the reconstructed top quark mass resolution. We describe here the additional corrections to these raw measured quantities and their uncertainties.

\subsubsection{Lepton}

An additional correction is applied to the muon track $p_{T}$ before applying the $\chi^{2}$ fit. The muon transverse momentum is corrected for the COT chamber misalignment:

$$
\frac{1}{p_{T}^{\mu}[\mathrm{GeV} / c]}=\frac{1}{p_{T}^{\mu}[\mathrm{GeV} / c]}-0.00037-0.00110 \sin (\phi+0.28)
$$


where $\phi$ is the azimuthal angle of the muon track. The uncertainty on the muon $p_{T}$ is given by the tracking resolution:

$$
\frac{\sigma_{p_{T}^{\mu}}^{\mu}}{p_{T}^{\mu}}=0.0011 \cdot p_{T}^{\mu}[\mathrm{GeV} / c] .
$$

The electron $p_{T}$ resolution is given by the EM calorimeter resolution:

$$
\frac{\sigma_{p_{T}^{e}}}{p_{T}^{e}}=\sqrt{\left(\frac{0.135}{\sqrt{p_{T}^{e}[\mathrm{GeV} / c]}}\right)^{2}+(0.02)^{2}}
$$

The masses of both the electrons and muons are set to zero in the fit as they are insignificant.

\section{$5.2 .2 \quad J e t s$}

The four highest $E_{T}$ jets are assumed to come from the four quarks resulting directly from the top quark and $W$ boson decays. We first apply to these jets the full generic jet energy corrections described in Sec. 4.2.4. Additional jet corrections are needed such that the measured jet energy is equal to the average energy of the corresponding quark from the $t \bar{t}$ decay chain. These top-specific corrections provide jet energy correction factors and resolutions separately for $W$-daughter jets and $b$-jets. These two are treated independently since $W$-daughter jets are due to the fragmentation of light quark jets while $b$-jets have different characteristics that affect their response: "harder" fragmentation, different colour flow in $t \bar{t}$ events and a larger fraction of semileptonic decays arising from the $B$-hadrons. The differences between the $W$ daughter jets and $b$-jets energy scale are discussed in more detail in Sec. 8.1. The top-specific corrections also account for the $p_{T}$-dependence of the $W$-daughter jets and $b$-jets response, arising from the combined effect of the non-flat $t \bar{t}$ jets $p_{T}$ spectrum and the finite jet energy resolution (often refer to as "feed-down" or "feed-up" effects).

The top-specific corrections are extracted from a HERWIG $t \bar{t}$ MC calculation with $M_{\text {top }}=178 \mathrm{GeV} / c^{2}$. Figure 5.1 shows the mean of the jet response (where the response is 

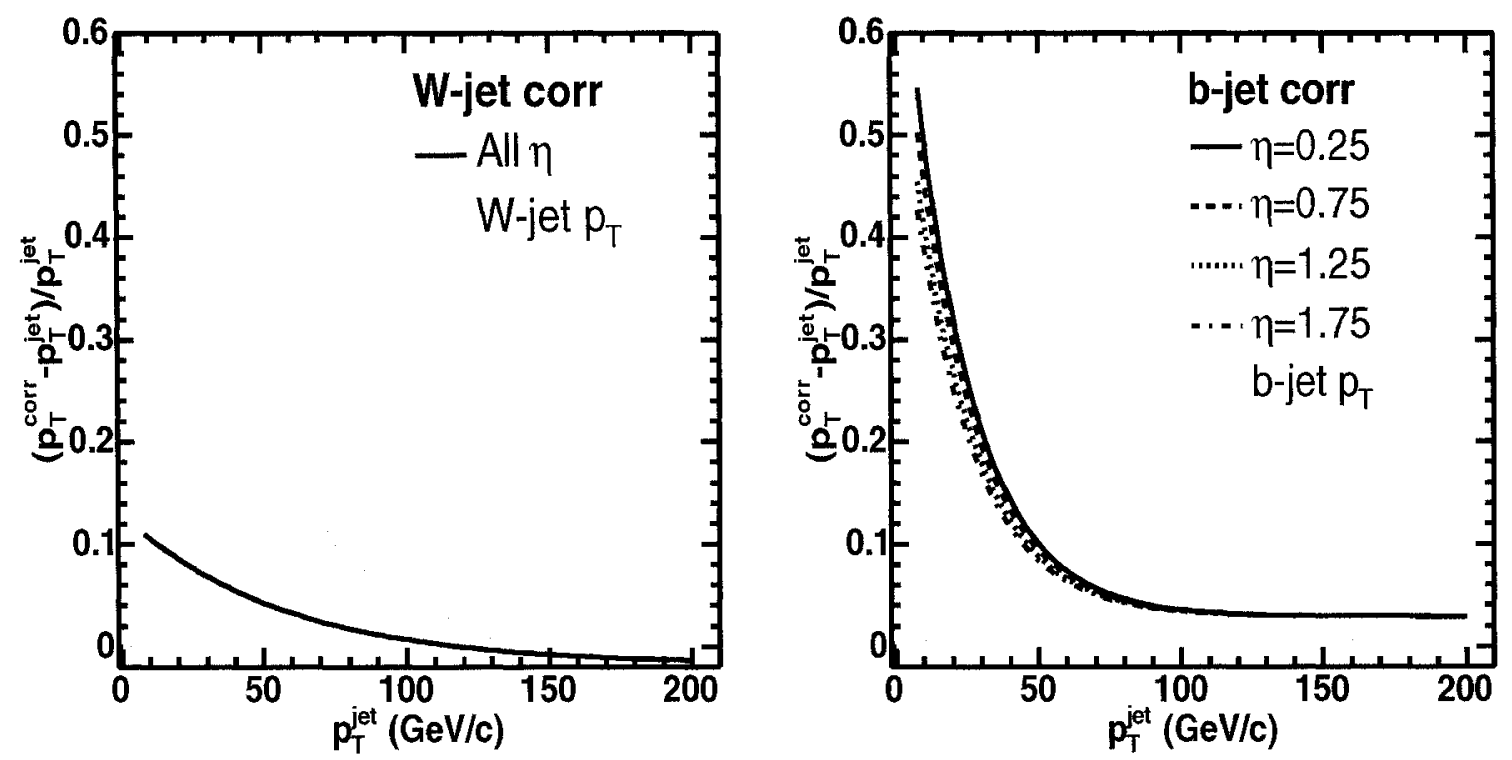

Figure 5.1: Correction factors of the top-specific corrections defined as the most probable value of the jet response function as a function of jet $p_{T}$ and $\eta$ (extracted from a HERWIG $t \bar{t}$ sample with $\left.M_{t o p}=178 \mathrm{GeV} / c^{2}\right)$. Overlaid on the histogram is the $p_{T}$ of the $t \bar{t}$ jets corrected with generic jet energy corrections.

defined as $\left.\frac{p_{T}(\text { quark })-p_{T}(j e t)}{p_{T}(j e t)}\right)$ as a function of the jet $p_{T}$ for various $\eta$ regions. The exhibited $p_{T}$-dependence is mostly due to the shape of the jet $p_{T}$ spectrum that is overlaid in Fig. 5.1. The $\eta$-dependence is due to the fact that $t \bar{t}$ events are produced near threshold at the Tevatron (as discussed in Chapter 2) and thus tend to be generated in the central region of the detector. ${ }^{1}$ Correction factors are extracted such that the $p_{T}$ of a corrected jet is equal on average to the most probable value (MPV) of the corresponding quark $p_{T}$. The MPV has been observed to yield smaller $m_{t}^{r e c o}$ resolution than using the mean or the median. The $\chi^{2}$ fit also uses the resolution of the jet $p_{T}$ measurement (the $\sigma_{i}$ in Eqn. 5.1). This is obtained by computing the width of the jet response function that covers $68 \%$ of the area under the curve around the MPV. The jet energy resolutions are shown in Fig. 5.2 as a function of jet $p_{T}$ and $\eta$.

${ }^{1}$ Although the observed $\eta$-dependence is not significant for the $W$-daughter jets as seen in Fig. 5.1. 

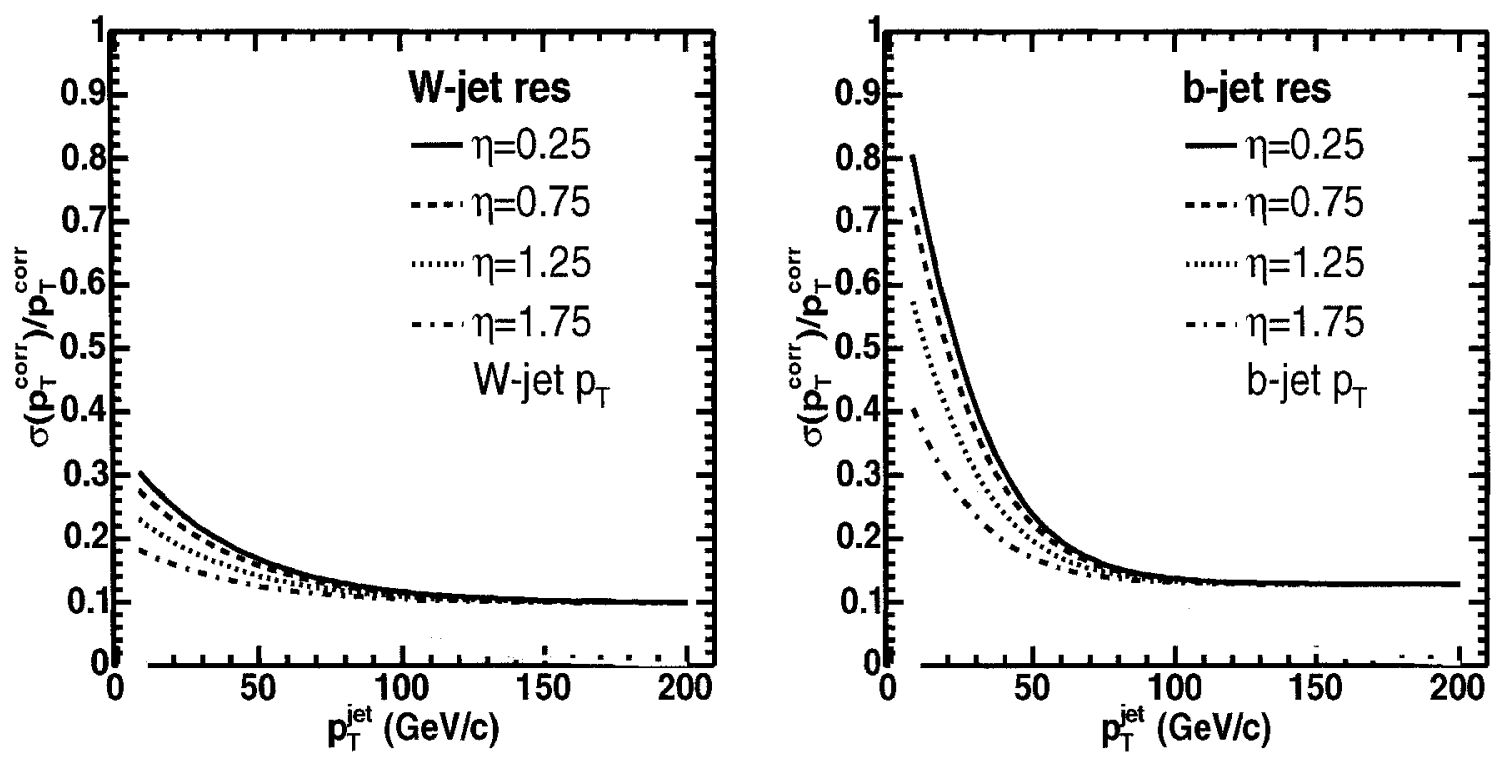

Figure 5.2: Jet $p_{T}$ resolution of the top-specific corrections defined as the width $(68 \%$ area under the curve) of the jet response function as a function of jet $p_{T}$ and $\eta$ (extracted from a HERWIG $t \bar{t}$ sample with $\left.M_{t o p}=178 \mathrm{GeV} / c^{2}\right)$. Overlaid on the histogram is the $p_{T}$ of the $t \bar{t}$ jets corrected with generic jet energy corrections.

More detail on the top-specific corrections can be found in [48]. To complete the calculation of the jet four-momentum, the masses of the $W$-daughter jets and $b$-jets are respectively set to $0.5 \mathrm{GeV} / c^{2}$ and $5.0 \mathrm{GeV} / c^{2}$ in the fit. It has been shown that the reconstruction of the top quark mass is mostly insensitive to the mass assignment of the jets $[49]$.

\subsubsection{Unclustered Energy}

The unclustered energy consists of all the energy that is not deposited by the lepton and the four highest $E_{T}$ jets in the calorimeters. It is used to infer the neutrino transverse momentum.

The raw unclustered transverse energy $U_{T}^{r a w}$ is defined as the raw energy measured in the calorimeter that is not due to the primary lepton or any jets with $p_{T}>8 \mathrm{GeV} / c$ 
and $|\eta|<2.0$. The corrected unclustered energy components $U_{i}, i=x, y$ are defined as

$$
U_{i}=1.4 * U_{i}^{r a w}+\sum_{j e t=1}^{4}\left[p_{i}^{u e}-p_{i}^{o o c}\right]+\sum_{j e t=5}^{n j e t s} p_{i}^{c o r r}, \quad i=x, y
$$

In the first term, the raw unclustered energy components $U_{i}^{\text {raw }}$ are corrected by a factor 1.4 that corresponds roughly to the full generic jet correction (defined in Sec. 4.2.4) for raw jets with $p_{T}=8 \mathrm{GeV} / c$. The second term adds to the unclustered energy the contribution from the underlying event inside the jet cone $\left(p_{i}^{u e}\right)$ and subtracts the energy out of the jet cone $\left(p_{i}^{o o c}\right)$ for the four highest $E_{T}$ jets, which are implicitly corrected for these effects by the top-specific corrections. The last term adds the energy of all additional jets in the event (corrected with full generic jet energy corrections) with $p_{T}>8 \mathrm{GeV} / c$ and $|\eta|<2.0$. With this definition, $U_{i}$ is a measure of all the energy flow in a lepton + jets $t \bar{t}$ event that is not due to the primary lepton and the four primary jets.

The uncertainty assigned to each component of the unclustered energy is related to the calorimeter resolution and has been measured to be

$$
\sigma^{U E}=0.4 * \sqrt{\sum E_{T}^{c a l}}
$$

where $\sum E_{T}^{c a l}$ is the total transverse energy recorded in the calorimeter in the event. Studies have shown that the variable $m_{t}^{\text {reco }}$ is mostly insensitive to variations in the definition of the unclustered energy or its associated uncertainty [49].

The only remaining object of a lepton+jets $t \bar{t}$ event that we have to account for is the neutrino. Its transverse momentum components are given by the missing transverse energy of the event

$$
p_{i}^{\nu}=-\left(p_{i}^{\ell}+\sum_{j e t=1}^{4} p_{i}^{j e t}+U_{i}\right), \quad i=x, y
$$

where $p_{i}^{\ell}$ and $p_{i}^{j e t}$ are, respectively, the fully corrected lepton and momentum components of the four highest $E_{T}$ jets and $U_{i}$ is given by Eqn. 5.5. As mentioned previously, the initial value of $p_{z}^{\nu}$ is inferred by assuming the reconstructed mass $m_{\ell \nu}=M_{W}$. The mass of the neutrino is set to 0 in the fit. 


\begin{tabular}{cc}
\hline \hline Subsample & $\chi_{\min }^{2}$ Cut Eff. \\
\hline 2-tag & 0.65 \\
1-tag(T) & 0.85 \\
1-tag(L) & 0.80 \\
$0-\operatorname{tag}$ & 0.92 \\
\hline \hline
\end{tabular}

Table 5.1: Signal efficiency for cut $\chi_{\text {min }}^{2}<9$ for $M_{t o p}=178 \mathrm{GeV} / c^{2}$.

\subsection{The $\chi_{\min }^{2}$ Cut}

The reconstructed top quark mass for the jet-parton assignment and $p_{z}(\nu)$ solution that yields the lowest $\chi^{2}$ value $\left(\chi_{\text {min }}^{2}\right)$ is kept for further analysis. We find that accepting only events with $\chi_{\min }^{2}<9$ improves the sensitivity of the measurement. Indeed, events with a lower $\chi_{\min }^{2}$ have a smaller reconstructed top quark mass resolution since they are more consistent with the $t \bar{t}$ hypothesis that is implicit in the $\chi^{2}$ fit. On the other hand, tightening the $\chi_{\min }^{2}$ cut reduces the $t \bar{t}$ signal acceptance. An optimization of the $\chi_{\min }^{2}$ cut has been performed and a value of 9 has been shown to perform well for all subsamples [50]. The signal efficiency for HERWIG $t \bar{t}$ Monte Carlo for $M_{t o p}=178 \mathrm{GeV} / c^{2}$ and nominal JES for the chosen cut are given in Table 5.1. The efficiencies decrease with increasing number of $b$-tags since there are fewer jet-parton assignments considered and thus less chance to have a combination with a low $\chi^{2}$.

The reconstructed top quark mass distributions are shown in Fig. 4.6 for each of the four subsamples after the $\chi_{m i n}^{2}$ cut is applied $\left(M_{t o p}=178 \mathrm{GeV} / \mathrm{c}^{2}\right)$. The mass distributions for the case where the $\chi^{2}$ fit picks the correct jet-parton assignment are overlaid. As can be seen, the fraction of events with the correct jet-parton assignment increases with the number of $b$-tags from $20 \%$ for 0 -tag events to $47 \%$ for 2 -tag events. 


\subsection{Definition of the Jet Energy Scale Uncertainty}

The templates of the reconstructed top quark and $W$ boson mass distribution are built for various values of the top quark mass and JES as described in Sec. 5.5 and 6.2, respectively. The parameter JES characterizes the deviation between the jet energy scale in $\mathrm{MC}$ and data events. The default jet energy scale in MC events is obtained by a tuning to the jet energy response observed in the data. The total uncertainty associated to this jet energy scale calibration is defined below and is denoted as $\pm 1 \sigma_{c}$. Using this definition, the jet energy scale in the tuned $\mathrm{MC}$ events corresponds to JES $=0 \pm 1 \sigma_{c}$.

The total $\pm 1 \sigma_{c}$ uncertainty on the JES calibration arises from potential sources of residual discrepancies between the energy response of jets in data and MC. Any such discrepancy can originate from the inherent complexity of the jet fragmentation and detection process, as illustrated in Fig. 5.3. We illustrate the magnitude of the uncertainties using the example of the "traditional" template top quark mass measurement [51] that does not use in situ $W \rightarrow j j$ decays to reduce the JES uncertainty. Variations of the total $\pm 1 \sigma_{c}$ JES uncertainty introduces an $M_{t o p}$ uncertainty of $3.1 \mathrm{GeV} / c^{2}$ in the traditional template analysis. The various components of the JES uncertainty are:

- Relative energy scale: Various parts of the CDF calorimeter have different scale uncertainties. Jets falling in the central calorimeter $\left(0.2 \lesssim \mid \eta_{\text {det }}(\right.$ jet $\left.) \mid \lesssim 0.9\right)$ are better understood than jets in transition regions between calorimeters that are not fully instrumented. Such transitions exist between the east and west side of the central calorimeter, the so-called $90^{\circ} \operatorname{cracks}\left(\left|\eta_{\operatorname{det}}(j e t)\right| \lesssim 0.2\right)$ and between the central and plug calorimeters, the so-called $30^{\circ}$ cracks $\left(0.9 \lesssim \mid \eta_{\text {det }}(\right.$ jet $) \mid \lesssim$ 1.4). Furthermore, the plug calorimeter region $\left(\mid \eta_{\text {det }}(\right.$ jet $\left.) \mid \gtrsim 1.4\right)$ is not as well understood as the central calorimeter since it does not benefit from in situ charged particle calibration. The systematic uncertainties are estimated using dijet event balancing described in Sec. 4.2.4, and the results are shown in Fig. 5.4 as a function 


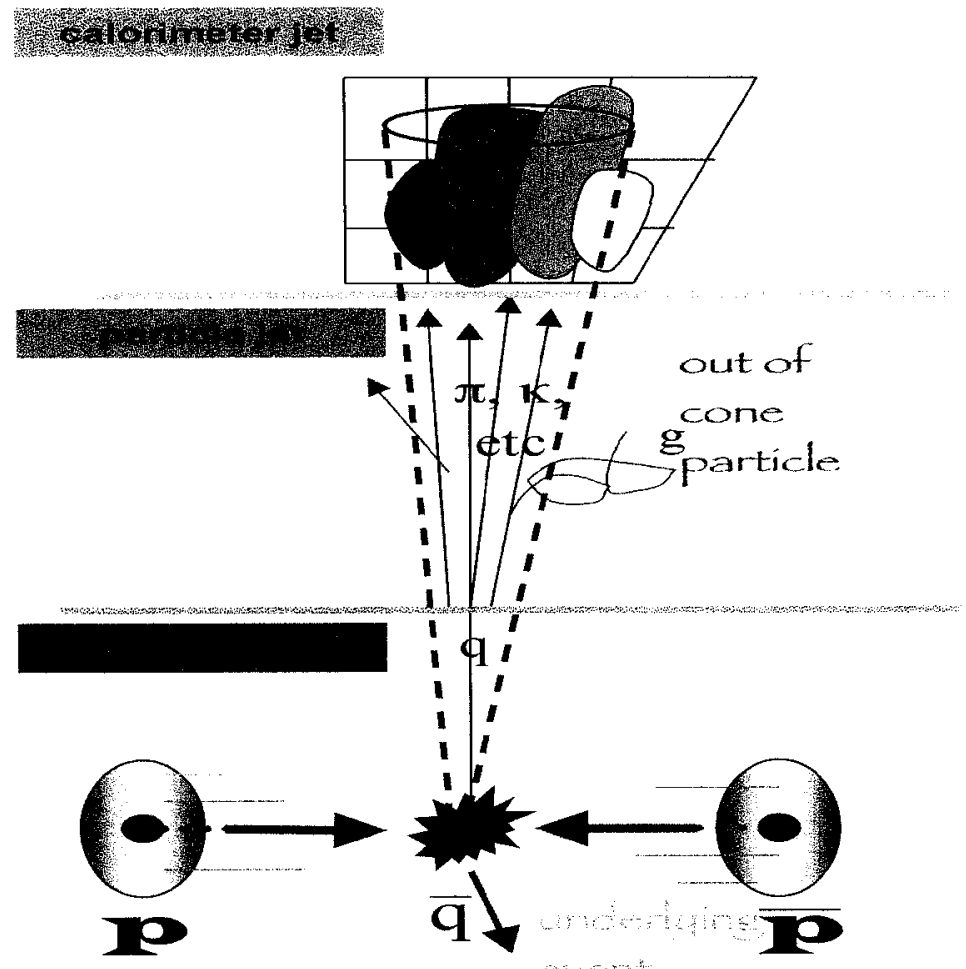

Figure 5.3: Sketch of the production and detection of a jet: a parton is produced out of the $p \bar{p}$ collision that hadronizes in neutral and charged particles of different species. Each of these particles interact in a different manner with the calorimeter. A cone algorithm is used to reconstruct the jet from the flow of energy detected in the calorimeter towers. Some of the initial parton energy is deposited outside the jet cone, while additional energy from the underlying event is deposited inside the jet cone.

of $\eta_{\text {det }}$ separated in five bins of jet $p_{T}$. This is a small uncertainty and contributes only $0.6 \mathrm{GeV} / c^{2}$ to the traditional template analysis.

- Absolute energy scale: The energy deposited inside the jet cone has uncertainties due to the calorimeter response of individual particles and the fragmentation of jets. The calorimeter response uncertainty is dominated by the limited knowledge of the non-linear $E / p$ response of hadronic particles. Even with a perfect understanding of the calorimeter, the detected energy depends on the fragmentation of jets, i.e. the distributions of the number of particles in a jet and their momentum spectra. 


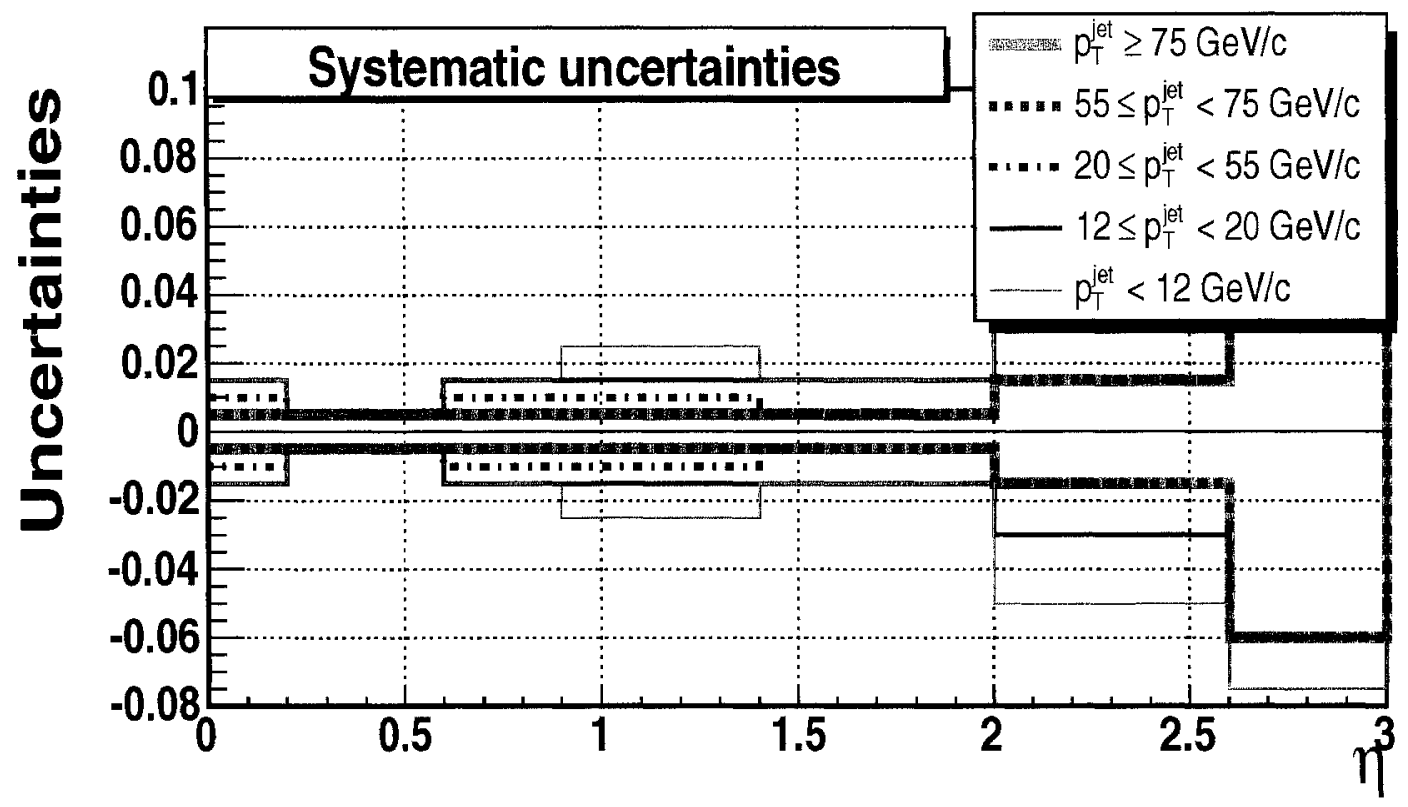

Figure 5.4: Relative jet energy systematic uncertainty as a function of $\left|\eta_{\text {det }}(j e t)\right|$ for $p_{T}($ jet $)<12 \mathrm{GeV} / c$ (solid pink), $12<p_{T}($ jet $)<20 \mathrm{GeV} / c$ (solid red), $20<$ $p_{T}($ jet $)<55 \mathrm{GeV} / c$ (dashed black), $55<p_{T}($ jet $)<75 \mathrm{GeV} / c$ (dashed blue) and $p_{T}($ jet $)>75 \mathrm{GeV} / c($ solid green $)$.

The fragmentation uncertainty contributes a $1 \%$ relative uncertainty on $p_{T}(j e t)$.

The absolute energy scale uncertainty also includes a small contributions from the stability of the calorimeter response over the data-taking period. Figure 5.5 shows the absolute scale uncertainty versus $p_{T}(j e t)$. This uncertainty contributes $2.2 \mathrm{GeV} / c^{2}$ to the traditional template analysis.

- Underlying event energy: The underlying event, that is energy from the remnants of the $p$ and $\bar{p}$ resulting from the hard scattering, can deposit energy inside the jet cone. This contribution has been measured to be $0.35 \pm 0.10 \mathrm{GeV}$ for a jet cone of radius $R=0.4$. The underlying event uncertainty is small compared to other contributions (only $0.3 \mathrm{GeV} / c^{2}$ for the traditional template analysis). The uncertainty on the modeling of the multiple interactions per bunch crossing has been 


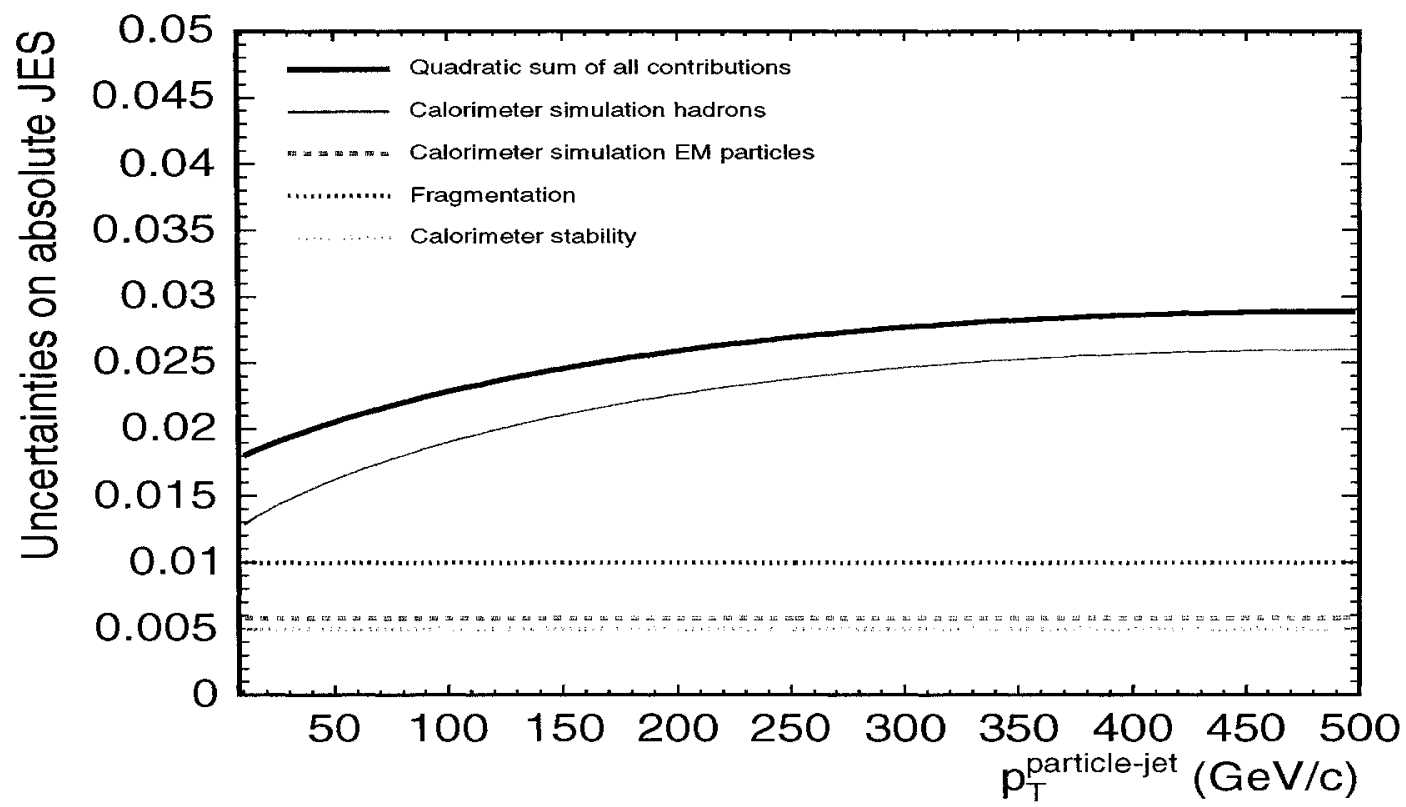

Figure 5.5: Absolute scale uncertainty from calorimeter response to electromagnetic (dashed pink) and hadronic particles (solid blue), jet fragmentation (dotted red), calorimeter stability over the running period (dotted green) and their contribution added in quadrature (solid black). The scale here is in relative uncertainty on the jet transverse momentum.

determined to be less than $0.1 \mathrm{GeV} / c^{2}[51]$ for the current dataset, and is therefore negligible in this analysis.

- Out-of-cone energy: A significant fraction of the jet energy is deposited outside the jet cone radius $\Delta R=0.4$. This amount of energy varies from approximately $20 \%$ for $p_{T}($ jet $)=20 \mathrm{GeV} / c$ to $5 \%$ for $p_{T}($ jet $)=100 \mathrm{GeV} / c$. The MC modeling of the out-of-cone energy has been compared to the data for photon + jets events and the results are shown in Fig. 5.6. The quoted uncertainty is overlaid in Fig. 5.6. This uncertainty contributes $2.2 \mathrm{GeV} / c^{2}$ to the traditional template analysis.

All sources are uncorrelated and added in quadrature to yield a total JES uncertainty. The total $\pm 1 \sigma_{c}$ uncertainty is shown in Fig. 5.7 for the central region $\left(0.2 \lesssim \mid \eta_{\text {det }}(\right.$ jet $) \mid \lesssim$ 0.6). The JES uncertainty is about $6 \%$ for $p_{T}(j e t)=20 \mathrm{GeV} / c$ and $3 \%$ for $p_{T}(j e t)=$ 


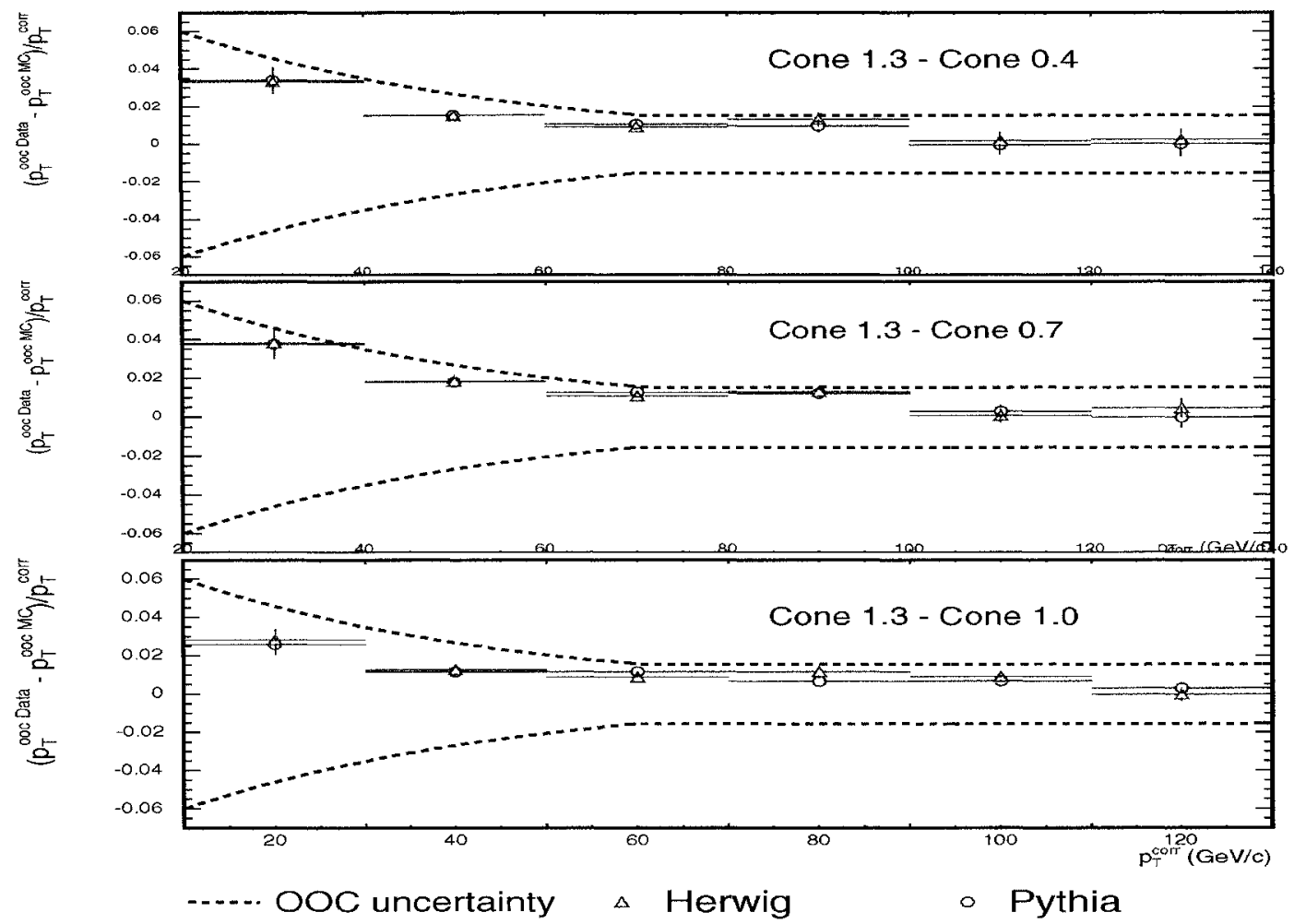

Figure 5.6: Systematic uncertainty from out-of-cone energy for cone $\Delta R=0.4$ (dashed black). Also shown are the comparisons in energy out-of-cone between data and Pythia (red circles) and Herwig (blue triangles) Monte Carlo in the region $\Delta R=0.4-1.3$ (upper plot), 0.7-1.3 (middle plot) and 1.0-1.3 (bottom plot).

$100 \mathrm{GeV} / c$. More detail on the jet energy calibration in CDF can be found in [45].

The parameter JES is expressed in terms of $\sigma_{c}$ in this analysis. This is a convenient choice because it allows a natural combination of the measurement of JES through the CDF calorimeters and the jet calibration procedure (JES $\left.=0 \pm 1 \sigma_{c}\right)$ and the in situ measurement from $W \rightarrow j j$ (as described further in Chapter 7). Furthermore, $\sigma_{c}$ is dependent on the jet $p_{T}$ and $\eta$, and thus yields more realistic uncertainties on a jet-by-jet basis than using jet energy scale variations that are common to all jets (e.g. shifting all jets by the same numerical scale factor). 


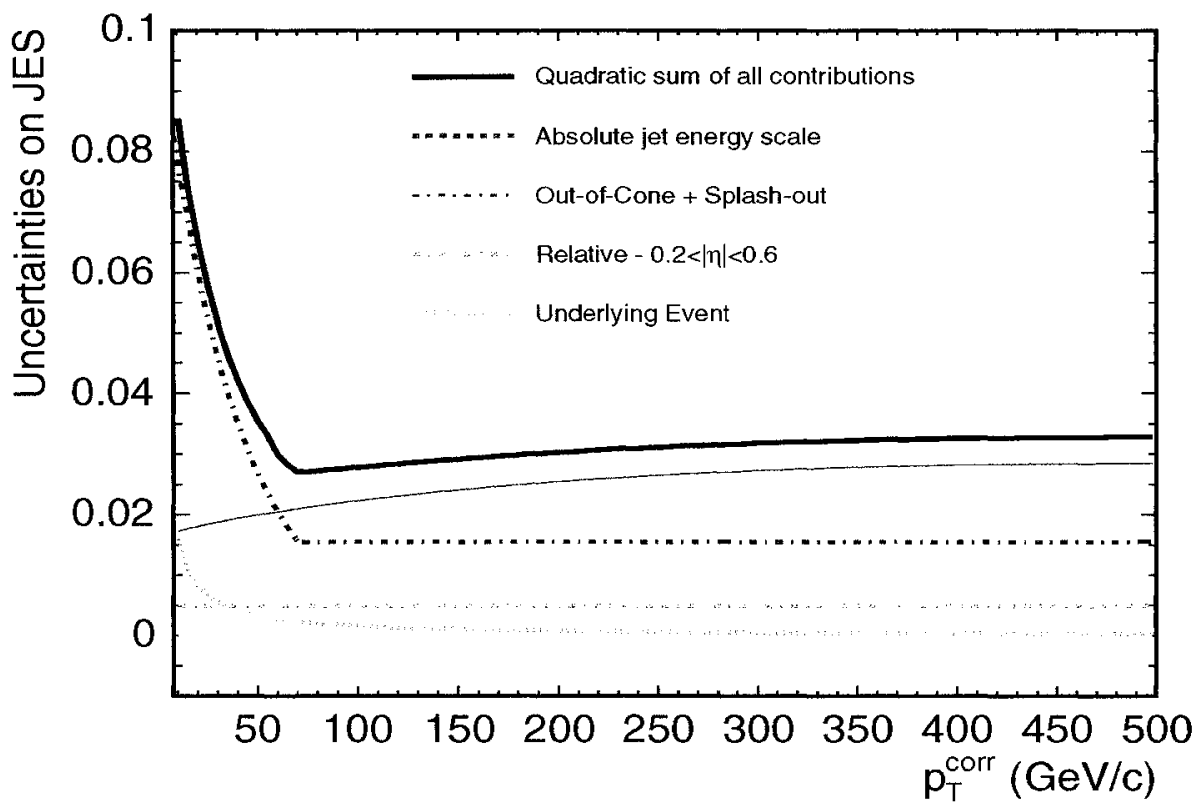

Figure 5.7: Total jet energy scale uncertainty $\left(+1 \sigma_{c}\right)$ as a function of the corrected jet $p_{T}$ (solid black). Also shown are the components from the underlying event (dotted blue), relative response (dashed green), out-of-cone energy (dashed red) and absolute response (solid blue).

\subsection{Signal Templates}

We produce reconstructed top quark mass distributions for 31 HERWIG Standard Model $t \bar{t}$ samples with the top quark mass ranging from $130 \mathrm{GeV} / c^{2}$ to $230 \mathrm{GeV} / c^{2}$. For each top quark mass, we produce templates for different jet energy scales: from $-3 \sigma_{c}$ to $+3 \sigma_{c}$ in steps of $0.5 \sigma_{c}$ where $\sigma_{c}$ is defined in the previous section. A subset of these templates is shown in Fig. 5.8 as a function of the top quark mass (for JES $=0 \sigma_{c}$ ) and in Fig. 5.9 as a function of JES (for $M_{t o p}=180 \mathrm{GeV} / c^{2}$ ) for the 1-tag(T) subsample. The signal acceptance changes as a function of $M_{t o p}$ and JES and this effect is taken into account in the shape of the templates.

These figures illustrate the anti-correlation between $M_{t o p}$ and JES for the $m_{t}^{\text {reco }}$ observable. For example, the $m_{t}^{\text {reco }}$ distribution for $M_{t o p}=190 \mathrm{GeV} / c^{2}, \mathrm{JES}=0 \sigma_{c}$ ( shown in Fig. 5.8) is similar to a $m_{t}^{\text {reco }}$ distribution with a lower mass but a higher JES, for instance 


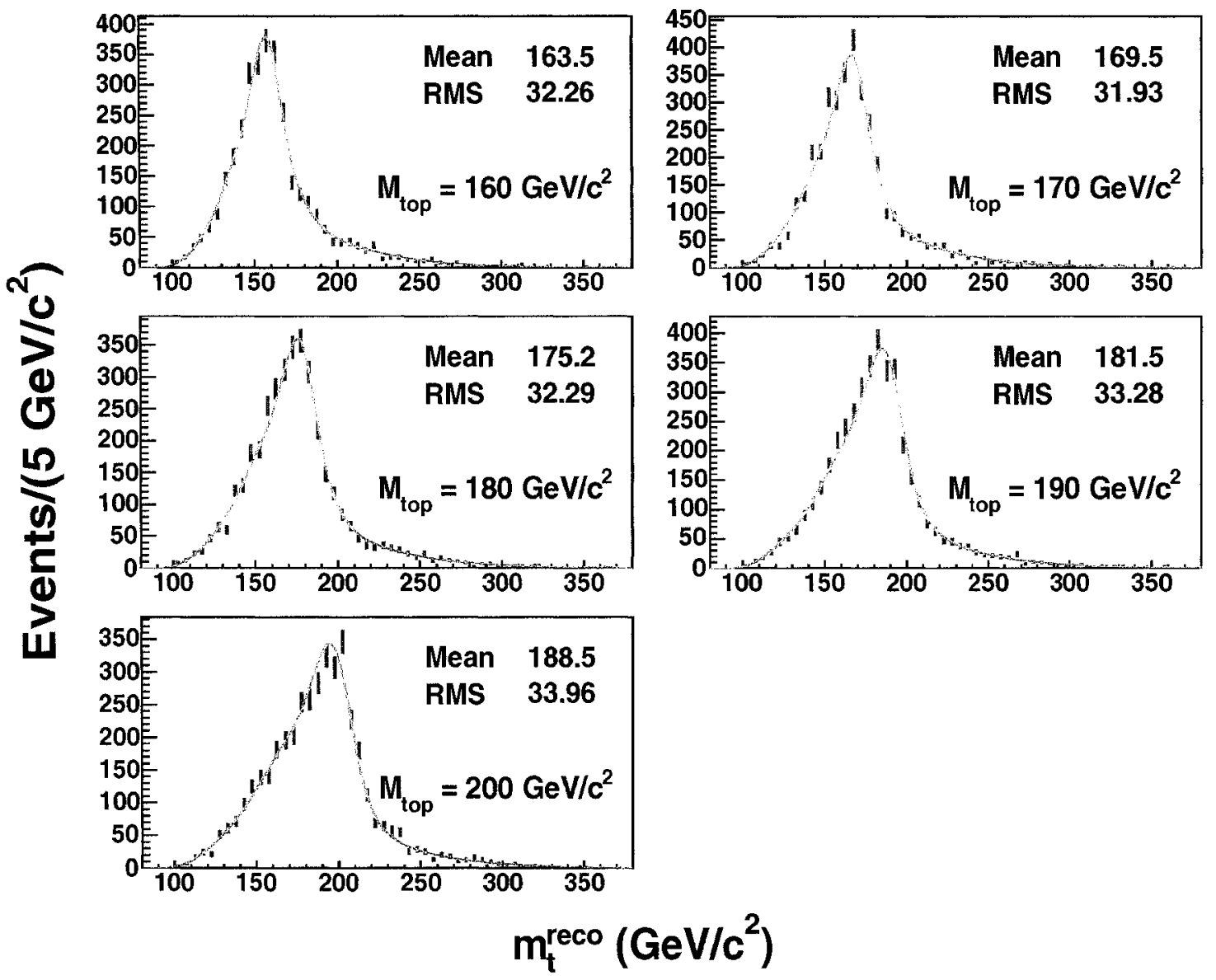

Figure 5.8: Reconstructed top quark mass distributions for various top quark mass (JES $=0 \sigma_{c}$ ) for the $1-\operatorname{tag}(\mathrm{T})$ subsample. The results of the template parameterizations are overlaid on top of the histograms.

$M_{\text {top }}=180 \mathrm{GeV} / c^{2}, \mathrm{JES}=1.5 \sigma_{c}$ (shown in Fig. 5.9). The JES systematic uncertainty on $M_{t o p}$ arises from this large correlation.

We obtain smooth probability density functions (p.d.f.'s) of the reconstructed top quark mass as a function of $M_{t o p}$ and JES by fitting the templates for each subsamples separately using two Gaussian functions and the integrand of a Gamma function as given by Eqn. 5.8. Each function parameter is linearly dependent on $M_{\text {top }}$ and JES as given 


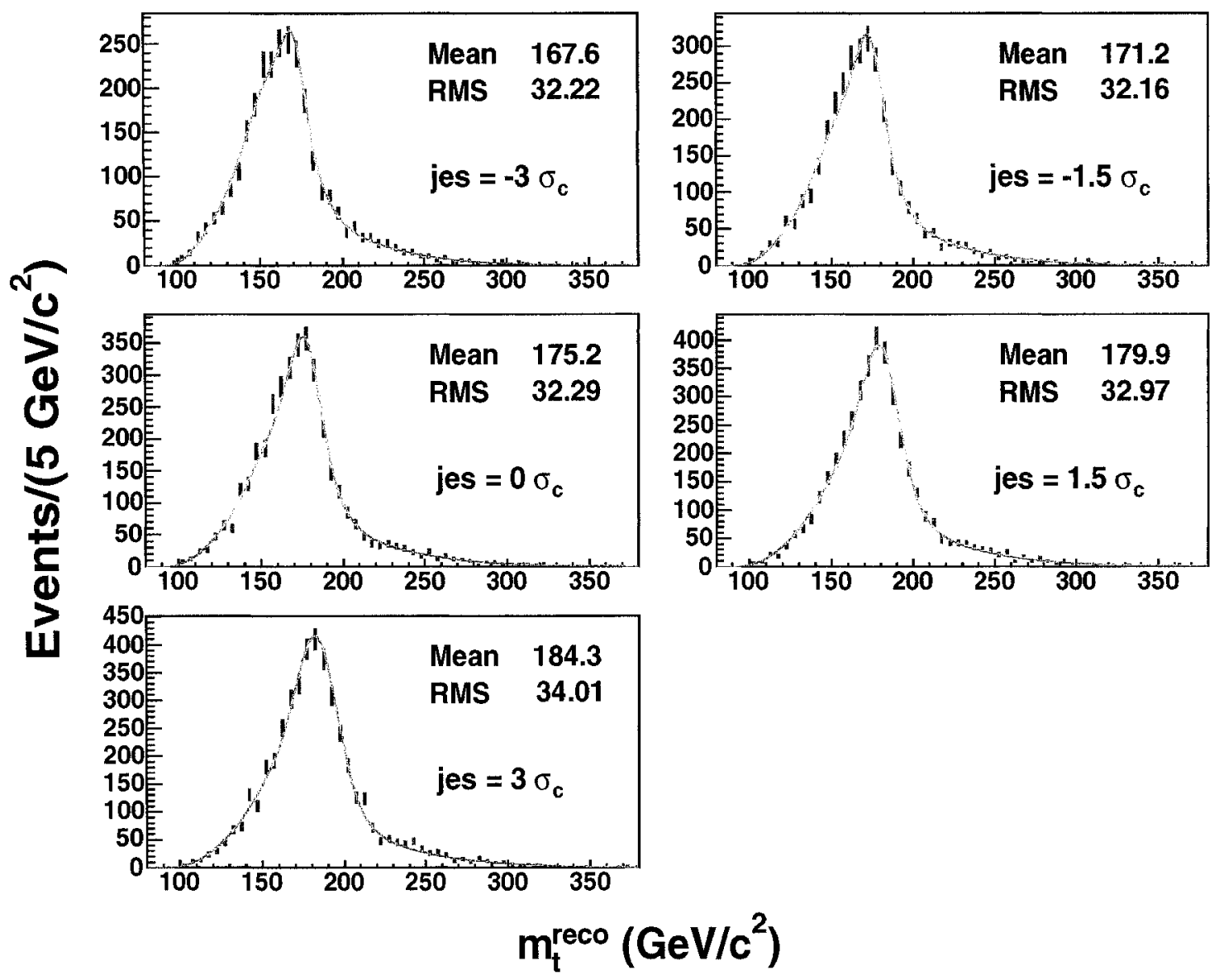

Figure 5.9: Reconstructed top quark mass distributions for various value of JES $\left(M_{t o p}=\right.$ $\left.180 \mathrm{GeV} / c^{2}\right)$ for the $1-\operatorname{tag}(\mathrm{T})$ subsample. The results of the template parameterizations are overlaid on top of the histograms.

by Eqn. 5.9:

$$
\begin{aligned}
P_{s i g}\left(m ; M_{t o p}, J E S\right)= & \alpha_{7} \cdot \frac{\alpha_{2}^{1+\alpha_{1}}}{\Gamma\left(1+\alpha_{1}\right)} \cdot\left(m-\alpha_{0}\right)^{\alpha_{1}} e^{-\alpha_{2}\left(m-\alpha_{0}\right)} \\
& +\alpha_{8} \cdot \frac{1}{\alpha_{4} \sqrt{2 \pi}} \cdot e^{\frac{-\left(m-\alpha_{3}\right)^{2}}{2 \alpha_{4}^{2}}} \\
& +\left(1-\alpha_{7}-\alpha_{8}\right) \cdot \frac{1}{\alpha_{6} \sqrt{2 \pi}} \cdot e^{\frac{-\left(m-\alpha_{5}\right)^{2}}{2 \alpha_{6}^{2}}}
\end{aligned}
$$

where

$$
\alpha_{i}=p_{i}+p_{i+9} \cdot\left(M_{t o p}-175\right)+p_{i+18} \cdot(J E S), \quad i=0,2, \ldots 8 .
$$

A quadratic dependence of the function parameters over $M_{t o p}$ and JES has been at- 


\begin{tabular}{cc}
\hline \hline Subsample & $\chi^{2} /$ ndof \\
& $m_{t}^{\text {reco }}$ templates \\
\hline 2-tag & $13459 / 13227$ \\
1-tag(T) & $18893 / 17194$ \\
1-tag(L) & $14392 / 13615$ \\
0-tag & $19091 / 16980$ \\
\hline \hline
\end{tabular}

Table 5.2: The $\chi^{2} / n d o f$ for the fit of Eqns. 5.8-5.9 to the $m_{t}^{\text {reco }}$ signal templates.

tempted but had only a negligible impact on the quality of the fit. The resulting $\chi^{2}$ per degrees of freedom $\left(\chi^{2} / n d o f\right)$ are given in Table 5.2. The $\chi^{2} / n d o f$ are in some cases significantly larger than one but the resulting parametrization, overlaid on the histogram of Figs. 5.8 and 5.9 for the $1-\operatorname{tag}(\mathrm{T})$ case, reproduces well the $m_{t}^{\text {reco }}$ distributions for large ranges of $M_{\text {top }}$ and JES. As will be discussed in Chapter 7, this parametrization is shown to yield unbiased estimates of $M_{t o p}$ and JES.

We note that even though the correct jet-parton assignments produce $m_{t}^{\text {reco }}$ mainly in the region from approximately $150 \mathrm{GeV} / c^{2}$ to $200 \mathrm{GeV} / c^{2}$ (as illustrated in Fig. 4.6), the full range of values of $m_{t}^{\text {reco }}$ is considered in the analysis. Indeed, the tails of the $m_{t}^{r e c o}$ distribution contain some information ${ }^{2}$ on $M_{t o p}$. Since this measurement is limited by statistical uncertainties (as will be clear in Chapter 8), we attempt to maximize the statistical power of the analysis by including all values of $m_{t}^{\text {reco }}$.

\subsection{Background Templates}

We need to take into account the background contamination of the $t \bar{t}$ sample to obtain an unbiased measurement of $M_{t o p}$, which requires a knowledge of the rate and shape of the background. The a priori calculation of the expected number of background events

\footnotetext{
${ }^{2}$ For instance the momentum of the jets is correlated to $M_{t o p}$.
} 
for each of the tagged subsamples is discussed in Sec. 4.4. In the current section, we describe the creation of the background $m_{t}^{\text {reco }}$ templates.

The reconstructed top quark mass shape for background events is obtained by applying the $m_{t}^{r e c o}$ reconstruction method described in Sec. 5.1 to the various background sources discussed in Sec. 4.4. We use $W b \bar{b} \mathrm{MC}$ events to model the $W$ theavy flavor background. The assumption that $W b \bar{b}$ events is a good model for $W c \bar{c}$ and $W c$ background events has been verified [49]. The $m_{t}^{\text {reco }}$ distribution of $W+$ jets events where at least one jet is mistagged as a $b$-jet ( $W+$ jets mistag) is obtained from a $W+$ jets $\mathrm{MC}$ sample where the SECVTX mistag probability, obtained from studying real data events, is applied to each jet of a given event in the $t \bar{t}$ sample before applying the $b$ tag criteria. The QCD multijets events are assumed to have the same mass shape as $W+$ jets mistag events for the tagged subsamples and $W+$ jets events for the 0-tag subsample. A systematic uncertainty arising from this assumption is included (see Sec. 8.6). The very small diboson background is assumed to be modeled by $W$ theavy flavor events. Single-top MC samples are used to obtain the single-top templates. The final background template is created by combining the templates of each source in the proportions given in Tab. 4.3. The resulting combined background $m_{t}^{\text {reco }}$ templates are shown in Fig. 5.10 for each subsample.

The background templates are independent of the top quark mass, but in principle could depend on JES. However, the dependence of the background mass shape over JES is very small. ${ }^{3}$ Instead, we choose to estimate the JES uncertainty on the background shape separately (as described in Chapter 8). The normalization of the background is also unaffected by JES since the dominant background sources ( $W+$ jets and QCD) are extracted using real data events.

An analytical description of the background $m_{t}^{r e c o}$ shape is obtained by fitting the

\footnotetext{
${ }^{3}$ This is because the background events are more susceptible to enter or leave the $t \bar{t}$ sample than signal events as the JES varies, because the jets in background events are less energetic on average, and thus closer to the jet $E_{T}$ cut.
} 
templates with two different functions. For the 0-tag background, two integrands of Gamma functions are needed:

$$
\begin{aligned}
P_{b}(m)= & p_{6} \frac{p_{2}^{1+p_{1}}}{\Gamma\left(1+p_{1}\right)} \cdot\left(m-p_{0}\right)^{p_{1}} e^{-p_{2}\left(m-p_{0}\right)} \\
& +\left(1-p_{6}\right) \frac{p_{5}^{1+p_{4}}}{\Gamma\left(1+p_{4}\right)} \cdot\left(m-p_{3}\right)^{p_{4}} e^{-p_{5}\left(m-p_{3}\right)}
\end{aligned}
$$

In the tagged background case, one integrand of a Gamma function is sufficient:

$$
P_{b}(m)=\frac{p_{2}^{1+p_{1}}}{\Gamma\left(1+p_{1}\right)} \cdot\left(m-p_{0}\right)^{p_{1}} e^{-p_{2}\left(m-p_{0}\right)} .
$$

The resulting parametrization is overlaid on the histograms of Fig. 5.10, demonstrating that the chosen function describes well the reconstructed mass distributions. 

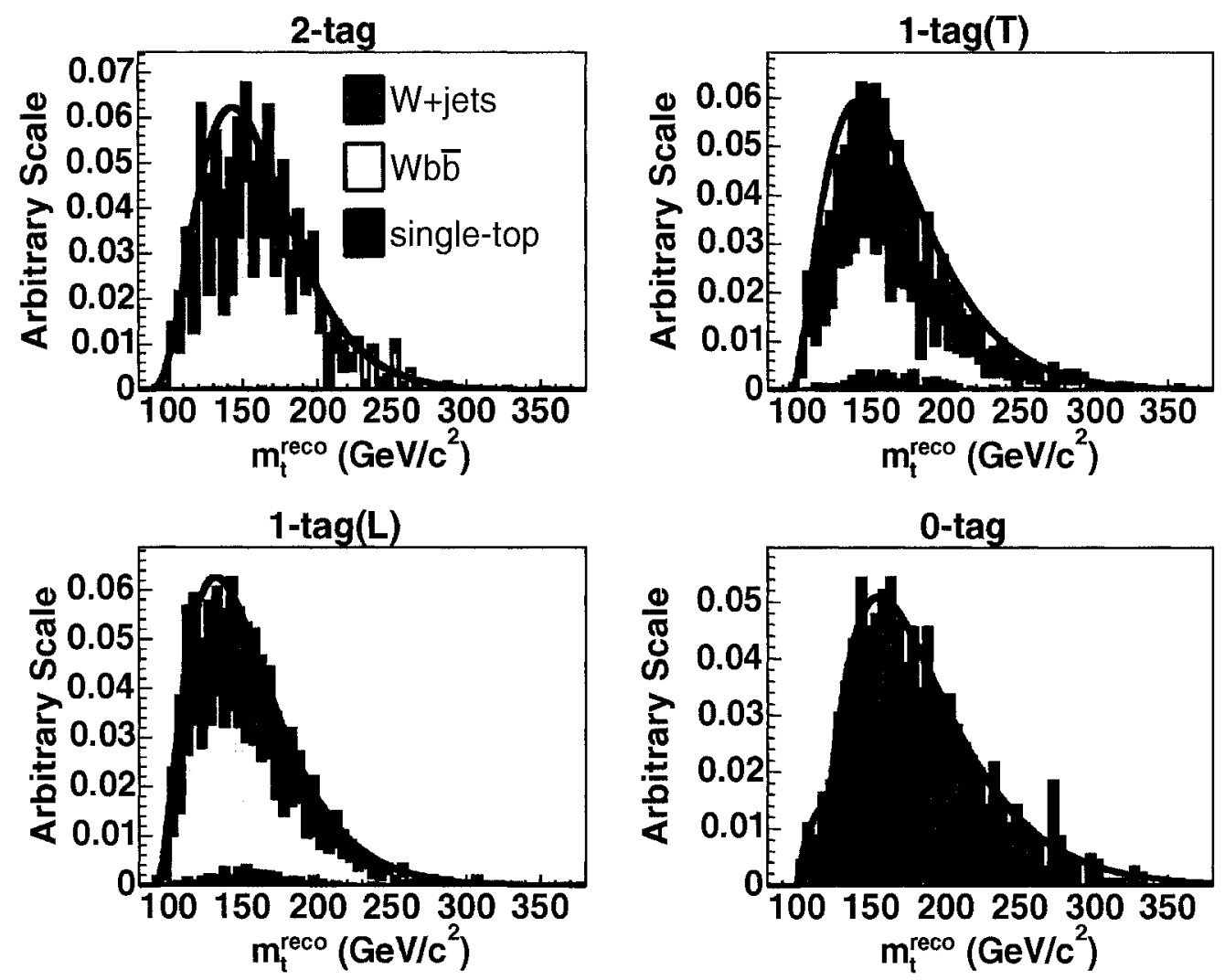

Figure 5.10: Combined background $m_{t}^{\text {reco }}$ templates for 2-tag events (upper-left), 1-tag(T) events (upper-right), 1-tag(L) events (bottom-left) and 0-tag events (bottom-right). The contributions from each background source are also illustrated in the 2-tag and 1-tag subsamples. 


\section{Chapter 6}

\section{W Boson Mass Reconstruction}

In this chapter we describe the reconstruction of the hadronic $W$ boson mass $m_{j j}$ that is used to measure JES. As will be noted, the $m_{j j}$ distributions are mostly insensitive to $M_{\text {top }}$, which allows for a determination of JES independent of $M_{t o p}$.

The reconstruction of the hadronically decaying $W$ boson mass is complicated by the ambiguity of correctly assigning jets in the final state to the initial state partons. We note however that the combinatorics problem is simpler than in the $m_{t}^{\text {reco }}$ case:

- Only the jets belonging to the light quarks from the $W$ decay need to be correctly assigned. Swapping the jets from the $b$ or $\bar{b}$ quark doesn't affect $m_{j j}$.

- We don't need to reconstruct the neutrino momentum. Thus, the quadratic ambiguity of the $p_{z}(\nu)$ solution has no impact on the $m_{j j}$ reconstruction.

Therefore, the number of combinations per event for 0-, 1-, 2-tag events are reduced from $24,12,4$ for the $m_{t}^{\text {reco }}$ reconstruction to $6,3,1$ for the $m_{j j}$ reconstruction, respectively.

\subsection{Reconstruction Method for $m_{j j}$}

In order to reconstruct $m_{j j}$, one has to identify the final state jets that result from the fragmentation of the $W$ daughter quarks. In this analysis, we do not attempt to determine 
uniquely the correct pair of jets and instead consider all jet-parton assignments made from the four highest $E_{T}$ jets with no $b$-tag and include them in the $m_{j j}$ templates. No fit is employed (unlike the $m_{t}^{r e c o}$ case) and the jets are corrected using the top-specific corrections for $W$-daughter jets described in Sec. 5.2.2. This method results in more than one mass combination per event. In fact, 1,3 and $6 m_{j j}$ per event are reconstructed for 2-, 1- and 0-tag events, respectively. The resulting $m_{j j}$ templates for each subsample made from HERWIG $t \bar{t}$ events with nominal JES and $M_{t o p}=178 \mathrm{GeV} / c^{2}$ are shown in Fig. 6.1. As for the $m_{t}^{\text {reco }}$ case, the fraction of correct jet-parton assignments (shown in the dark blue histogram in Fig. 6.1) increases with the number of $b$-tags present in the event. Therefore, events with two $b$-tags have a much better mass resolution ( $\mathrm{RMS}=36 \mathrm{GeV} / c^{2}$ ) than events with no $b$-tags ( $\left.\mathrm{RMS}=53 \mathrm{GeV} / c^{2}\right)$.

No $\chi_{\min }^{2}$ cut is applied in the $m_{j j}$ reconstruction (described in Sec. 5.3 for the $m_{t}^{\text {reco }}$ reconstruction) as applying such a cut reduces the precision of the JES measurement. This cut tends to select events with $m_{j j}$ close to the nominal $W$ boson mass even for large JES shifts, thus reducing the sensitivity of $m_{j j}$ to JES.

We note that an alternative method has been examined to reconstruct $m_{j j}$ that consist in using the $\chi^{2}$ kinematic fitter described in Sec. 5.1 where one or both $W$ mass constraints are removed. The jet-parton assignment that yields the lowest $\chi^{2}$ is declared the $m_{j j}$ for that event. The performance of both methods have been assessed and the method that considers all jet-parton assignments has been chosen for its better performances $(>20 \%$ better a priori JES statistical uncertainty than $\chi^{2}$ fit method). This advantage can be explained by two factors:

- The $m_{j j}$ distributions reconstructed from the $\chi^{2}$ kinematic fit have a mass resolution about $10 \%$ better. However, the mass distributions reconstructed by considering all jet-parton assignments shift by larger values for the same variations in JES (25\% more), resulting in a greater sensitivity.

- The correct combination for events in which the $W$-daughter jets are one of the 

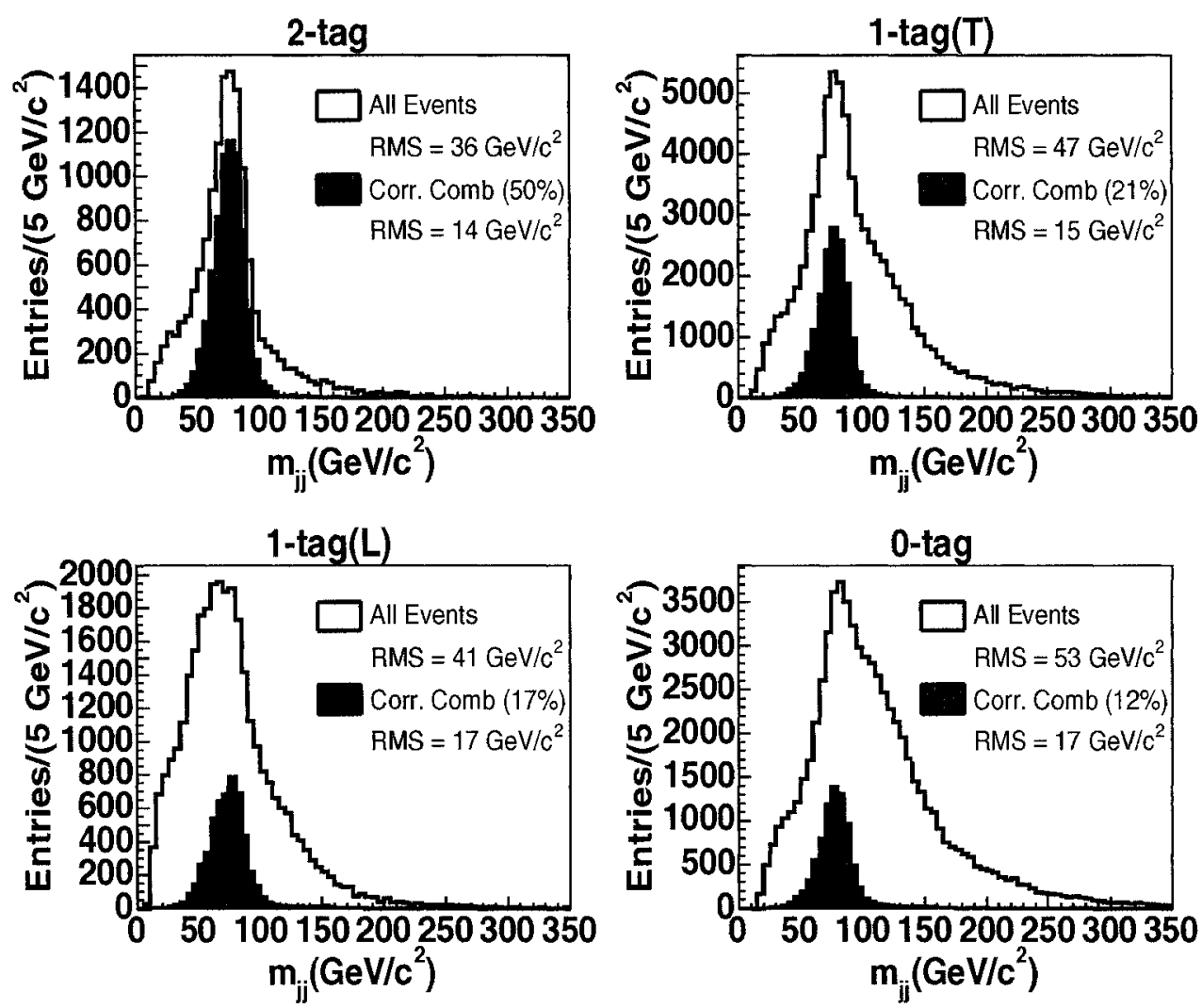

Figure 6.1: Reconstructed hadronic $W$ boson mass from HERWIG $t \bar{t}$ events with nominal JES and $M_{t o p}=178 \mathrm{GeV} / \mathrm{c}^{2}$ for 2-tag events (upper-left), 1-tag(T) events (upper-right), 1-tag $(\mathrm{L})$ events (bottom-left) and 0-tag events (bottom-right). The yellow (outer) histograms show the mass distributions for all events and the blue (inner) histograms show the distributions only for the correct jet-parton assignments.

four highest jets but for which the $\chi^{2}$ fitter makes an assignment error are still considered by the method that includes all jet-parton assignments.

Given the superior statistical power of the method that consider all jet-parton assignments to reconstruct $m_{j j}$, and since the JES measurement is dominated by statistical uncertainties (as will be clear in Chapter 8), we employ that technique in this measurement. 


\subsection{Signal Templates}

In a similar manner as for the $m_{t}^{r e c o}$ templates, the $m_{j j}$ templates are reconstructed as a function of JES and $M_{t o p}$, as shown for the 2-tag subsample in Figs. 6.2 and 6.3, respectively. As can be seen in these figures, the $m_{j j}$ distributions display some anti-correlation between $M_{t o p}$ and JES. For example, the distributions for $M_{t o p}=180 \mathrm{GeV} / c^{2}, \mathrm{JES}=1.5 \sigma_{c}$ (shown in Fig. 6.2) is similar to a distribution with a higher mass but smaller JES (for instance $M_{t o p}=200 \mathrm{GeV} / c^{2}, \mathrm{JES}=0 \sigma_{c}$, shown in Fig. 6.3). However, this correlation is small enough to extract JES with relatively small uncertainties from $M_{t o p}$. We note that these distributions are created with a true $W$ boson mass of $80.42 \mathrm{GeV} / c^{2}$ that is the default in HERWIG.

The templates are parametrized with the same function as the $m_{t}^{\text {reco }}$ templates given by Eqns. 5.8-5.9. The resulting $\chi^{2} / n d o f$ of the fit is given in Tab. 6.1. The $\chi^{2} / n d o f$ are significantly larger than one but the resulting parametrization, overlaid on the histogram of Figs. 6.2 and 6.3 for the 2-tag case, describes well the $m_{j j}$ shape for large ranges of $M_{t o p}$ and JES. The two-dimensional visualization of the $m_{j j}$ p.d.f.'s for the 2-tag subsample as a function of JES and $M_{\text {top }}$ are shown in Figs. 6.4 and 6.5, respectively. We note again that the $m_{j j}$ distribution is mostly independent of $M_{t o p}$, as shown in Fig. 6.5 but varies significantly as a function of JES as seen in Fig. 6.4.

Similarly to the $m_{t}^{\text {reco }}$ templates, we consider the full range of values of $m_{j j}$ in this analysis even though the masses of the correct jet-assignments are limited to the region from approximately $50 \mathrm{GeV} / c^{2}$ to $100 \mathrm{GeV} / c^{2}$ (as illustrated in Fig. 6.1). The tails of the $m_{j j}$ distribution are sensitive to JES (as illustrated in Fig. 6.4) but are also sensitive to $M_{t o p}$ (as illustrated in Fig. 6.5) that is a systematic uncertainty for the determination of JES. Since the JES measurement is limited by statistical uncertainties (as will be clear in Chapter 8), we thus attempt to maximize the statistical power of the analysis by including all values of $m_{j j}$. 


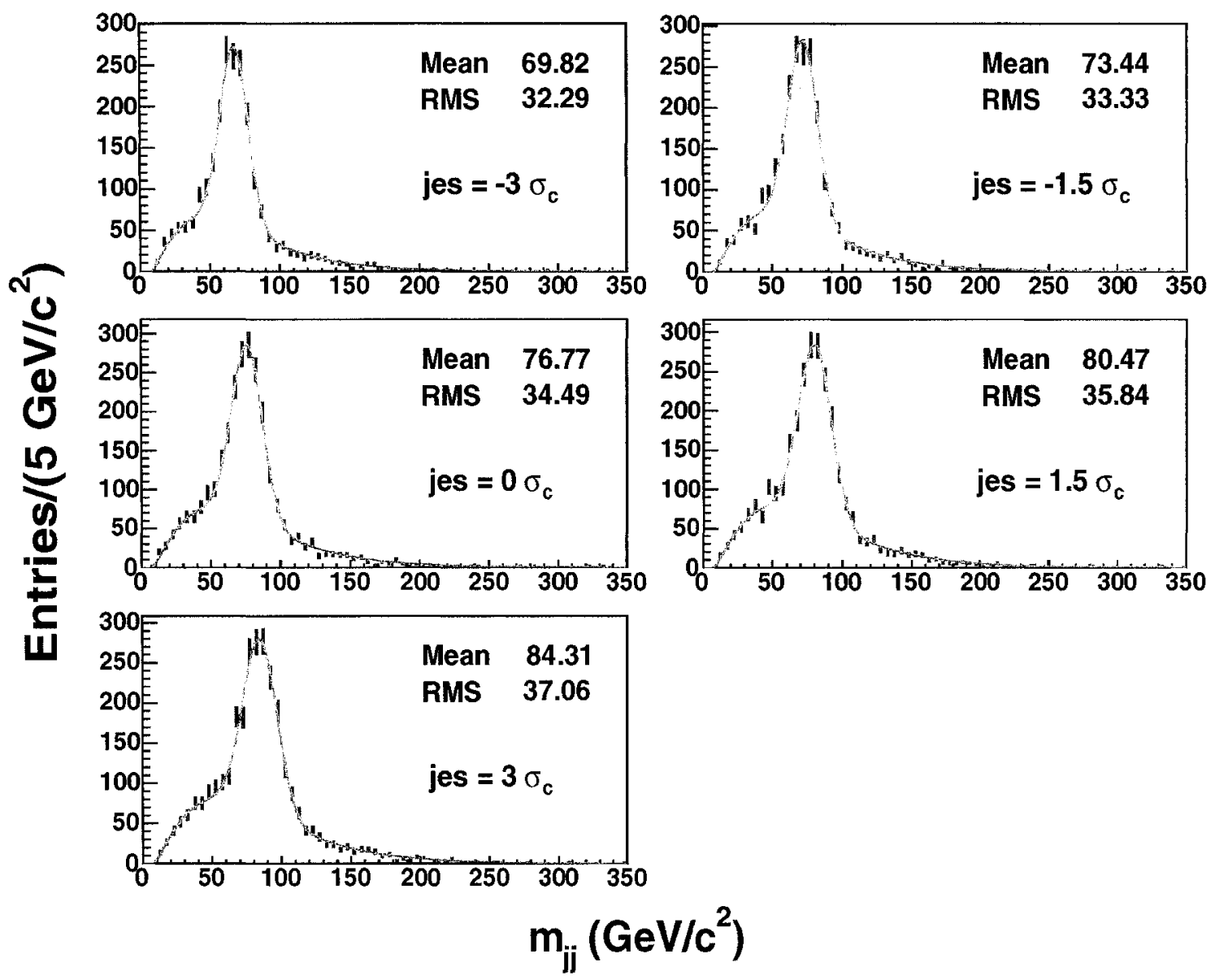

Figure 6.2: Reconstructed $W \rightarrow j j$ mass distributions for various value of JES $\left(M_{t o p}=\right.$ $180 \mathrm{GeV} / c^{2}$ ) for the 2-tag subsample. The results of the template parametrization are overlaid on top of the histograms.

\subsection{Background Templates}

The $m_{j j}$ background templates are reconstructed in the same manner as for the $m_{t}^{\text {reco }}$ background templates described in Sec. 5.6. The sources of background events are added in the proportions given in Table 4.2 for the $m_{j j}$ background. As for the $m_{t}^{\text {reco }}$ case, the $m_{j j}$ background templates are independent of $M_{t o p}$ and JES. The background templates for each subsample are shown in Fig. 6.6.

The parametrization of the $m_{j j}$ background templates is performed using a function made of one integrand of a Gamma function and two Gaussian function for the 0-tag 


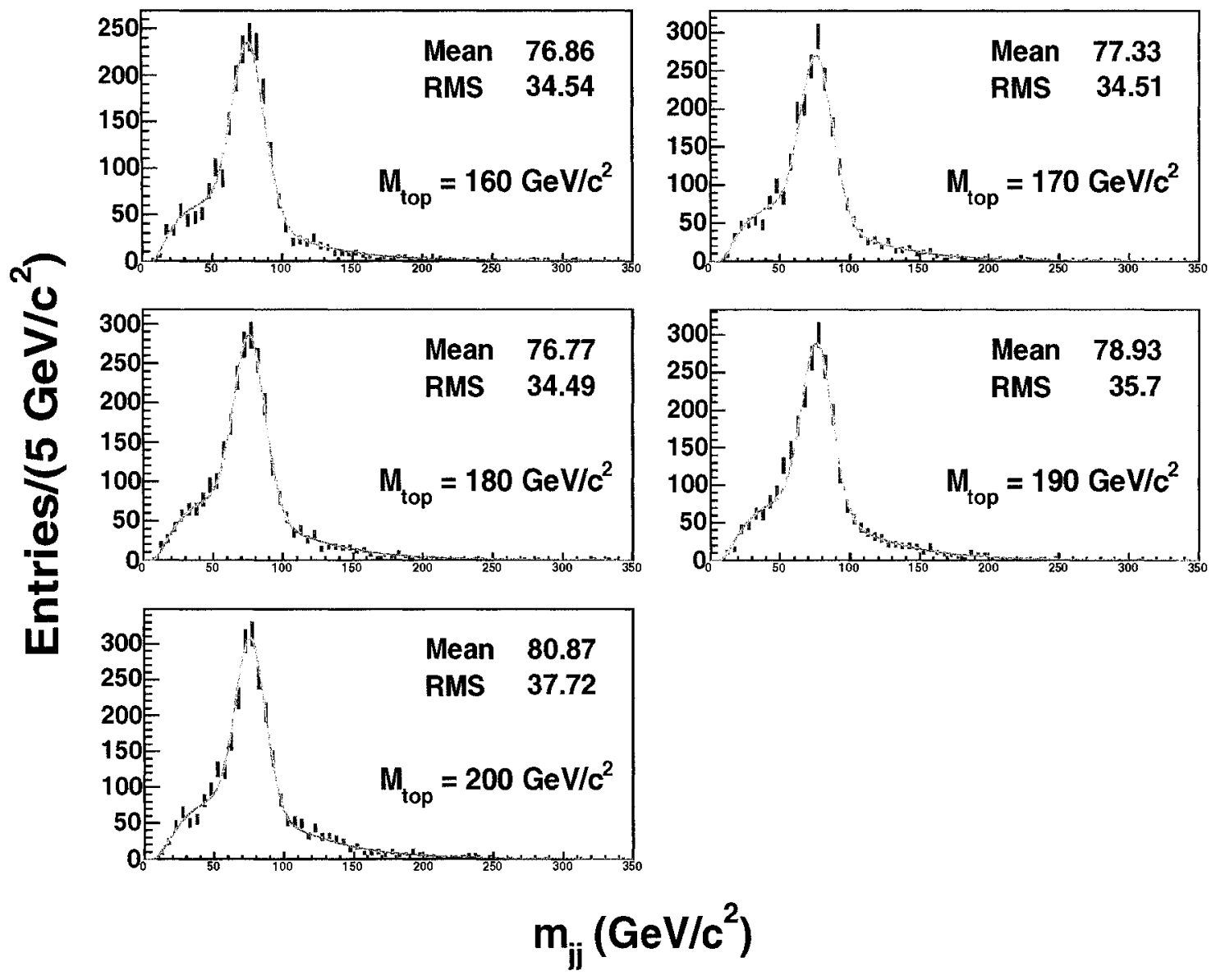

Figure 6.3: Reconstructed $W \rightarrow j j$ mass distributions for various top quark masses (nominal JES) for the 2-tag subsample. The results of the template parametrization are overlaid on top of the histograms.

background:

$$
\begin{aligned}
P_{b}(m)= & \alpha_{7} \cdot \frac{\alpha_{2}^{1+\alpha_{1}}}{\Gamma\left(1+\alpha_{1}\right)} \cdot\left(m-\alpha_{0}\right)^{\alpha_{1}} e^{-\alpha_{2}\left(m-\alpha_{0}\right)} \\
& +\alpha_{8} \cdot \frac{1}{\alpha_{4} \sqrt{2 \pi}} \cdot e^{\frac{-\left(m-\alpha_{3}\right)^{2}}{2 \alpha_{4}^{2}}} \\
& +\left(1-\alpha_{7}-\alpha_{8}\right) \cdot \frac{1}{\alpha_{6} \sqrt{2 \pi}} \cdot e^{\frac{-\left(m-\alpha_{5}\right)^{2}}{2 \alpha_{6}^{2}}}
\end{aligned}
$$

For the tagged background, one integrand of a Gamma function and one Gaussian func- 


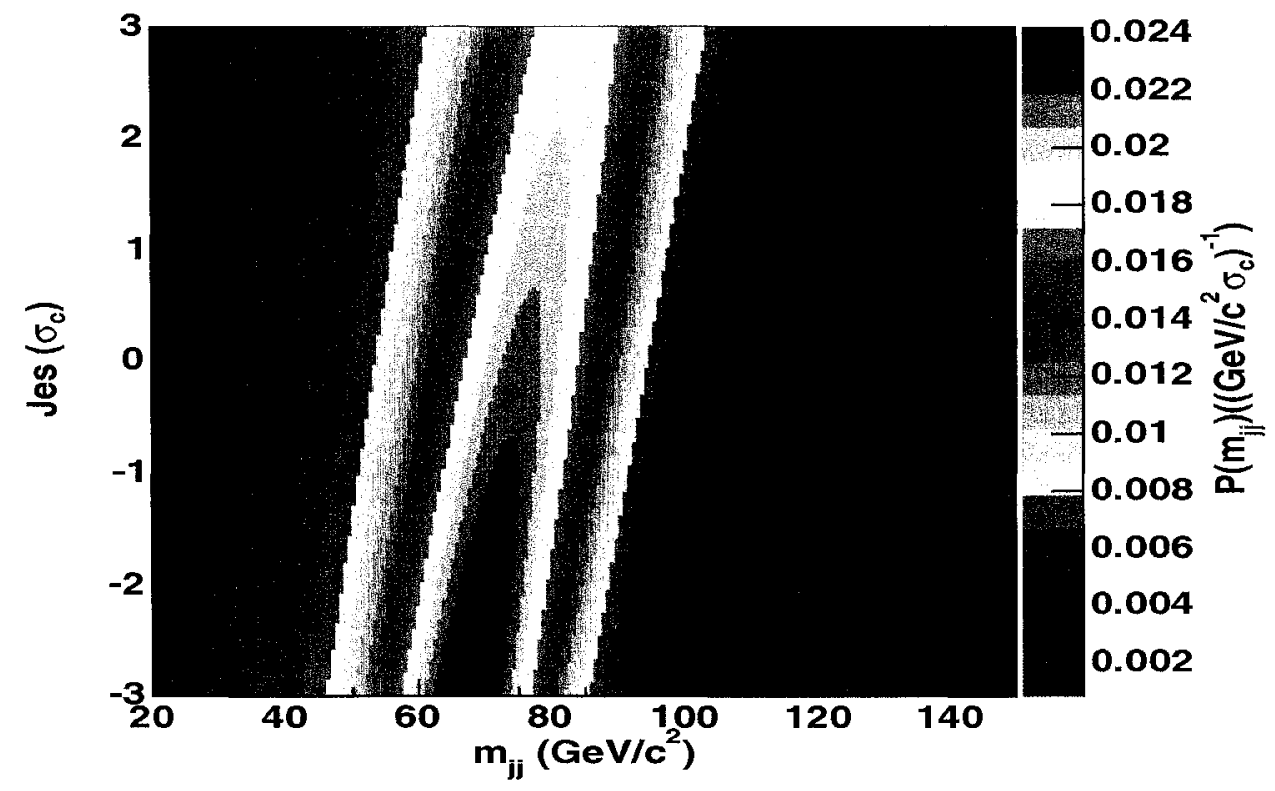

Figure 6.4: Probability density function of $m_{j j}\left(\mathrm{P}\left(m_{j j}\right)\right)$ versus $m_{j j}$ and JES for the 2-tag subsample $\left(M_{t o p}=180 \mathrm{GeV} / c^{2}\right)$.

tion are sufficient:

$$
\begin{aligned}
P_{b}(m)= & \alpha_{5} \cdot \frac{\alpha_{2}^{1+\alpha_{1}}}{\Gamma\left(1+\alpha_{1}\right)} \cdot\left(m-\alpha_{0}\right)^{\alpha_{1}} e^{-\alpha_{2}\left(m-\alpha_{0}\right)} \\
& +\left(1-\alpha_{5}\right) \cdot \frac{1}{\alpha_{4} \sqrt{2 \pi}} \cdot e^{\frac{-\left(m-\alpha_{3}\right)^{2}}{2 \alpha_{4}^{2}}} .
\end{aligned}
$$

The results of the parametrization are overlaid on the histograms of Fig. 6.6, demonstrating that the chosen function describes well the reconstructed mass distribution.

In summary, we use two kinematic variables $m_{t}^{\text {reco }}$ and $m_{j j}$ to measure simultaneously $M_{\text {top }}$ and JES. The observable $m_{t}^{r e c o}$ is designed to be sensitive to the top quark mass $M_{\text {top }}$. However, it also exhibits a significant dependence on JES. The second variable $m_{j j}$ is constructed to have maximal sensitivity to JES while at the same time being mostly independent on $M_{t o p}$. Analytical p.d.f.'s of these variables as a function of $M_{t o p}$ and JES have been obtained using HERWIG $t \bar{t}$ events. 


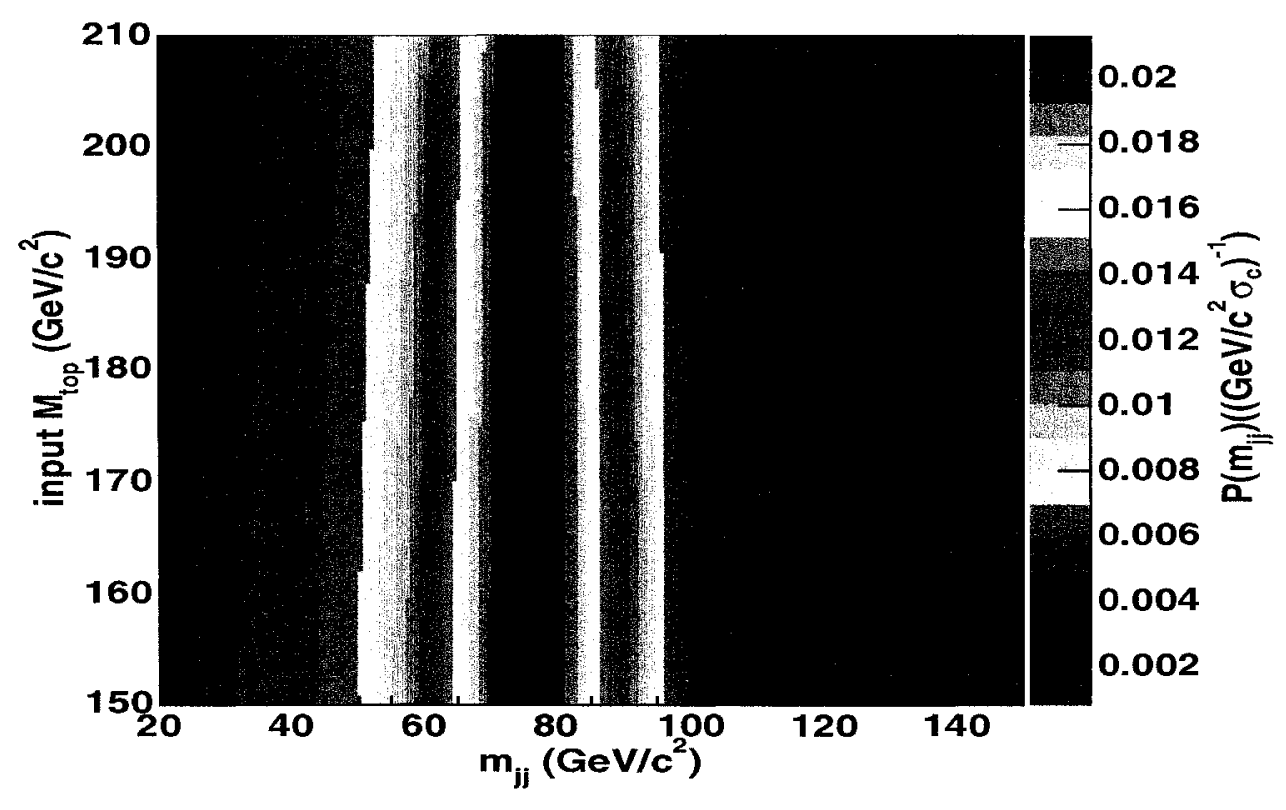

Figure 6.5: Probability density function of $m_{j j}\left(\mathrm{P}\left(m_{j j}\right)\right)$ versus $m_{j j}$ and $M_{t o p}$ for the 2-tag subsample (nominal JES).

\begin{tabular}{cc}
\hline \hline Subsample & $\chi^{2} /$ ndof \\
& $m_{j j}$ templates \\
\hline 2-tag & $19839 / 17186$ \\
1-tag(T) & $29401 / 25833$ \\
1-tag(L) & $23820 / 20039$ \\
0-tag & $38528 / 30794$ \\
\hline \hline
\end{tabular}

Table 6.1: The $\chi^{2} / n d o f$ for the fit of Eqns. 5.8-5.9 to the $m_{j j}$ signal templates. 

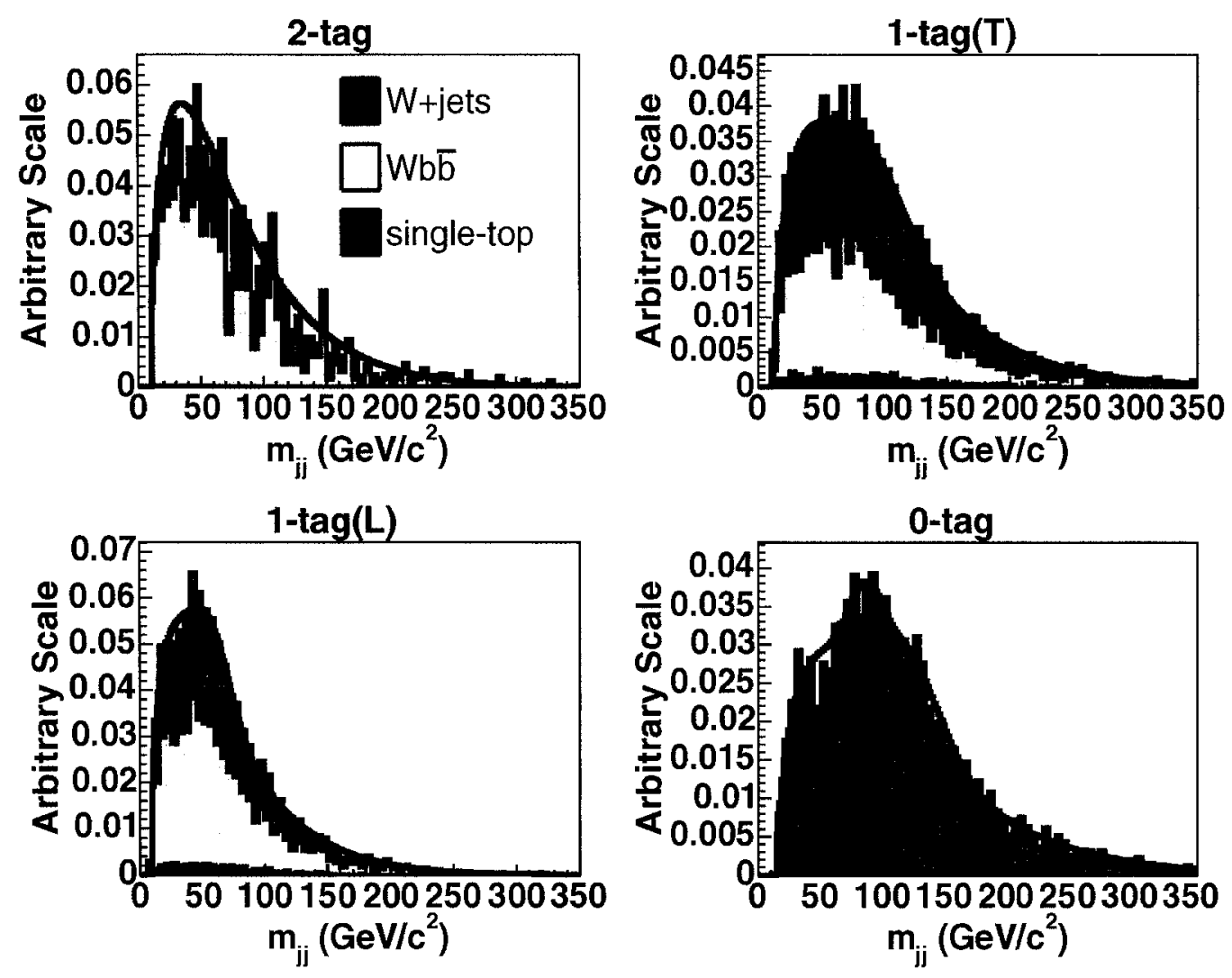

Figure 6.6: Combined background $m_{j j}$ templates for 2-tag events (upper-left), 1-tag(T) events (upper-right), 1-tag(L) events (bottom-left) and 0-tag events (bottom-right). The contributions from each background source are also illustrated in the 2-tag and 1-tag subsamples. 


\section{Chapter 7}

\section{Likelihood Fits}

\subsection{General Strategy}

The top quark mass is extracted from a likelihood fit that compares data $m_{t}^{\text {reco }}$ and $m_{j j}$ distributions to MC templates built with various hypotheses of $M_{t o p}$ and JES. The parameter JES is constrained by two measurements:

1. The CDF calibration of JES described in Sec. 5.4. Since the simulation is tuned such that the jet energy response is compatible with the data, the default jet energy scale in MC events corresponds to JES $=0 \pm 1 \sigma_{c}$, where the unit of JES uncertainty $\sigma_{c}$ is defined in Sec. 5.4.

2. The $m_{j j}$ templates that provide an in situ measurement of JES in $t \bar{t}$ events.

We acknowledge that these two sources of information on JES should not necessarily agree with each other. Indeed, the a priori JES calibration is obtained from events like dijets and photon + jet events that differ from $t \bar{t}$ events in ways that can potentially affect the jet energy scale:

- Jet flavor composition: The jets in photon+jet and dijets events are made of a mixture of gluon and quark jets while $t \bar{t}$ decay daughters are only quark jets. 
- Colour flow: The jets that come from the decay of the colour singlet $W$ boson suffer different colour effects than jets in dijets or photon+jet events.

- Calorimeter occupancies and charged particle multiplicities: These are higher in $t \bar{t}$ candidate events that contain at least four jets in the lepton+jets channel.

Therefore we first check that both measurements of JES are in agreement with each other before combining them for the final $M_{\text {top }}$ measurement. To do so, we measure JES in the data using only the $m_{j j}$ templates (with a fixed value of $M_{t o p}$ ) and check that the result is in agreement with the calibration result, JES $=0 \pm 1 \sigma_{c}$. This procedure is referred to as the "JES cross-check."

If the JES cross-check is successful, the measurement of the top quark mass using the full information from the $m_{t}^{r e c o}$ and $m_{j j}$ templates is performed. Both measurements of JES are combined to get an optimal constraint on this parameter. This procedure is referred to as the " $M_{t o p}$ measurement."

The likelihood fits used to perform the JES cross-check and $M_{\text {top }}$ measurement are described in this Chapter. We also check that both likelihood fits yield unbiased $M_{t o p}$ and JES central values and uncertainties using a pseudo-experiment procedure.

\subsection{Jet Energy Scale Cross-Check}

\subsubsection{Description of the Likelihood Fit}

For each subsample, we maximize the following likelihood function:

$$
\mathcal{L}_{\text {sample } i}=\mathcal{L}_{\text {shape }}^{m_{j j}} \times \mathcal{L}_{\text {Poisson }} \times \mathcal{L}_{\text {bg }}
$$

where,

$$
\begin{gathered}
\mathcal{L}_{\text {shape }}^{m_{j j}}=\prod_{k=1}^{r^{W} \times n_{i}^{\text {comb }}} \frac{n_{s}^{W} P_{\text {sig }}\left(m_{k}^{j j} ; J E S\right)+n_{b}^{W} P_{b}\left(m_{k}^{j j}\right)}{n_{s}^{W}+n_{b}^{W}} \\
\mathcal{L}_{\text {Poisson }}=\frac{e^{-\left(n_{s}^{W}+n_{b}^{W}\right)}\left(n_{s}^{W}+n_{b}^{W}\right)^{r^{W}}}{r^{W !}}
\end{gathered}
$$




$$
\mathcal{L}_{b g}=\exp \left(-\frac{\left(n_{b}^{W}-n_{b}^{W}(\text { constr })\right)^{2}}{2 \sigma_{n_{b}^{W}}^{2}}\right) .
$$

The function is maximized with respect to the three parameters JES, $n_{s}^{W}$ and $n_{b}^{W}$ where $n_{s}^{W}$ and $n_{b}^{W}$ are, respectively, the expectation value for the number of signal and background events in the $m_{j j}$ sample. The core of the information resides in $\mathcal{L}_{\text {shape }}^{m_{j j}}$ that calculates the product probability that a given reconstructed $W$ boson mass $m_{k}^{j j}$ comes from background $\left(P_{b}\left(m_{k}^{j j}\right)\right)$ or from signal given a jet energy scale JES $\left(P_{s i g}\left(m_{k}^{j j} ; J E S\right)\right)$. The expression for $P_{s i g}\left(m_{k}^{j j} ; J E S\right)$ is given in Eqns. 5.8-5.9 where in the case of the JES cross-check the top quark mass is fixed to the world average value $M_{\text {top }}=178.0 \mathrm{GeV} / \mathrm{c}^{2}$ [6]. The expressions for $P_{b}\left(m_{k}^{j j}\right)$ are given in Eqns. 6.1 and 6.2 for the 0-tag and $\geq 1$-tag subsamples, respectively. The multiplication is over the total number of observed events in the $m_{j j}$ sample, $r^{W}$, multiplied by the number of $m_{j j}$ per event, $n_{i}^{c o m b}$, for the given subsample $i$ (as explained previously there are 6,3 and $1 m_{j j}$ values per event for the 0-tag, 1-tag and 2-tag subsamples, respectively).

The second term represents the Poisson probability that the observed number of events, $r^{W}$, fluctuated from the Poisson mean given by the sum of the signal and background expectation values $n_{s}^{W}$ and $n_{b}^{W}$.

The third term is a Gaussian probability that constrains $n_{b}^{W}$ to the calculated a priori value $n_{b}^{W}$ (constr) within the uncertainty $\sigma_{n_{b}^{W}}$, where $n_{b}^{W}$ (constr) and $\sigma_{n_{b}^{W}}$ are given in Table 4.2. The signal expectation value $n_{s}^{W}$ is left unconstrained.

The final likelihood function is given by the product of the likelihood functions of all four subsamples:

$$
\mathcal{L}_{J E S}=\mathcal{L}_{2-\operatorname{tag}} \times \mathcal{L}_{1-\operatorname{tag}(T)} \times \mathcal{L}_{1-\operatorname{tag}(L)} \times \mathcal{L}_{0-\operatorname{tag}}
$$

The procedure uses simultaneously the information of all subsamples in the fit. The most probable value of JES is obtained by maximizing the final likelihood function with respect to all other parameters. We use the $\Delta \ln \mathcal{L}=0.5$ rule to define the one standard deviation uncertainty of JES, i.e. it corresponds to the value of JES where the logarithm 
of the likelihood has decreased by half a unit with respect to the most probable value (where the likelihood is re-optimized away from the maximum with respect to all other parameters, in this case $n_{s}^{W}$ and $n_{b}^{W}$ ). The uncertainty on the background normalization is reflected on the JES uncertainty. Since the likelihood function can be asymmetric, we allow for unequal absolute values of the positive and negative uncertainties. As will be described in the next section, we find that this definition does not provide complete coverage, i.e. the one standard deviation of JES defined above does not include the true value $68.3 \%$ of the time, and we need to derive a scale factor to inflate the uncertainties. The MINUIT package [52] is used to maximize the likelihood function.

\subsubsection{Pseudo-Experiments Procedure}

We check that the likelihood fit described above yields unbiased central values and uncertainties of JES using a large number of pseudo-experiments. The pseudo-experiments are generated in the following manner:

1. The number of signal and background events per experiment are drawn randomly from a Poisson distribution of the expected number of signal and background events in each subsample. The expected total number of signal events is calculated from the central value of the theoretical cross-section $\left(\sigma_{t \bar{t}}=6.1 \mathrm{pb}[18]\right)$, the detector acceptance and selection efficiencies calculated from a HERWIG Monte Carlo sample with $M_{t o p}=178 \mathrm{GeV} / c^{2}$ and the integrated luminosity of the dataset $\int \mathcal{L} d t=$ $318 \mathrm{pb}^{-1}$, and is given in Table 7.1 for each subsample. The expected number of background events is given in Table 4.2 for the tagged background. The expected number of background events in the 0-tag sample is given by the number of observed events (given in Table 4.1) minus the number of expected signal events.

2. The reconstructed masses $m_{j j}$ are generated randomly from the appropriate signal and background mass distributions (for a given input JES for the signal). The 


\begin{tabular}{ccc}
\hline \hline Subsample & \multicolumn{2}{c}{$n_{t \bar{t}}$} \\
& $m_{j j}$ & $m_{t}^{\text {reco }}$ \\
\hline 2-tag & 20.1 & 13.1 \\
$1-\operatorname{tag}(\mathrm{T})$ & 38.1 & 32.4 \\
$1-\operatorname{tag}(\mathrm{L})$ & 15.6 & 12.5 \\
$0-\operatorname{tag}$ & 21.2 & 19.4 \\
\hline \hline
\end{tabular}

Table 7.1: Expected number of signal events $n_{t \bar{t}}$ in the $m_{t}^{\text {reco }}$ and $m_{j j}$ samples. The difference between the $m_{j j}$ and $m_{t}^{\text {reco }}$ samples is the $\chi_{m i n}^{2}$ cut given in Table 5.1.

number of signal and background $m_{j j}$ to be generated is determined at the first step.

3. The central value of the background constraint $\mathcal{L}_{b g}$ is randomly generated according to a Gaussian distribution with corresponding mean and uncertainty. We do so to obtain normally distributed pull distributions in a constrained fit as recommended in [53].

4. The likelihood function described above is maximized, yielding a JES central value and uncertainties.

This procedure is used to test the viability of the likelihood fit and to estimate the systematic uncertainties. Typically several thousand pseudo-experiments are generated.

\subsubsection{Assessment of the Likelihood Performance}

We test the likelihood fit for input JES ranging from $-3 \sigma_{c}$ to $+3 \sigma_{c}$ in $1 \sigma_{c}$ intervals. At each JES value, 2,500 pseudo-experiments are generated. We check that the central values and uncertainties of JES obtained from the likelihood fit are appropriate by computing 
the pull distributions that are defined as:

$$
\text { Pull }=\left\{\begin{array}{lll}
\frac{J E S_{f i t}-J E S_{i n p u t}}{\sigma_{J E S_{f i t}}^{+}} & : & J E S_{f i t}<J E S_{\text {input }} \\
\frac{J E S_{f i t}-J E S_{i n p u t}}{\sigma_{J E S_{f i t}}^{-}} & : & J E S_{f i t}>J E S_{\text {input }}
\end{array}\right.
$$

where $J E S_{\text {fit }}$ is the fitted jet energy scale for a given pseudo-experiment, $J E S_{\text {input }}$ is the input jet energy scale and $\sigma_{J E S_{f i t}}^{+}$and $\sigma_{J E S_{f i t}}^{-}$are the positive and negative uncertainties, respectively. The mean and width of the pull distributions are extracted from a Gaussian fit. The left plot of Fig. 7.1 shows the mean of the pull distributions as a function of the input JES. The pull means are distributed around 0, though there appears to be a systematic trend with the pull mean falling with increasing input JES, and then rising again for positive JES. The maximal deviation is for JES $=-3 \sigma_{c}$ where the pull mean shows a bias of about $15 \%$ of the uncertainty, corresponding to a small bias of $\approx-0.15 \sigma_{c}$. The widths of the pull distributions as a function of the input JES are shown on the right plot of Fig. 7.1. The pull widths are distributed around $1.027 \pm 0.005$. The fact that the pull widths are larger than one is understood as arising from the relatively small statistics available, resulting in a non-Gaussian shape for the likelihood functions. A thorough investigation of the underestimation of the fit uncertainties in the template technique in the context of the top quark mass measurement is described elsewhere [49]. The uncertainties on JES returned by the likelihood fit are inflated by 1.027 in this analysis.

The statistical uncertainty on JES is shown as a function of the input JES in Fig. 7.2. At nominal JES, the uncertainty is about $1.00 \sigma_{c}$ (after multiplying the uncertainty by the scale factor of 1.027). Therefore, the JES statistical uncertainty extracted from the $W \rightarrow j j$ measurement is comparable to the one of the JES calibration with the current dataset $^{1}$. Figure 7.2 shows that the uncertainty in JES rises with the input value of JES.

\footnotetext{
${ }^{1}$ This can be achieved with a much smaller dataset than the one used for the JES calibration because most measurements in the latter case are systematically limited. For instance, this is the case for the largest uncertainty in the $p_{T}$ regime of interest for top physics, the out-of-cone energy.
} 

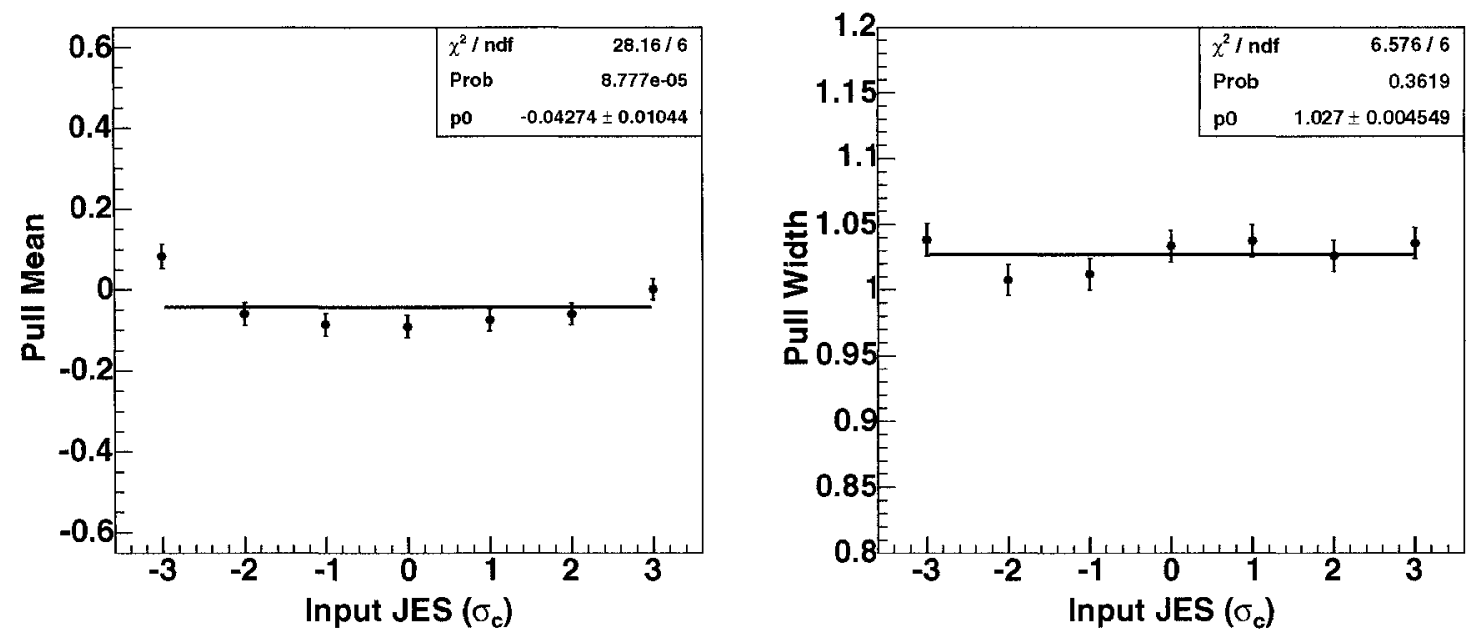

Figure 7.1: Mean (left plot) and width (right plot) of the JES pull distributions as a function of the input JES for the JES cross-check.

This is apparently due to the change in the relative width of the $m_{j j}$ distributions (and resulting statistical power) as JES increases. The expected weight of each subsample in the combined JES uncertainty is $32.6 \%, 44.7 \%, 10.4 \%$ and $12.3 \%$ for the 2 -tag, 1-tag(T), 1-tag(L) and 0-tag subsamples, respectively.

We conclude that the likelihood fit for the JES cross-check behaves well for a large range of input jet energy scale. We do not correct for the mean of fitted JES as any biases are less than $15 \%$ of the statistical uncertainty, but will inflate the JES uncertainty by 1.027. The expected statistical uncertainty is $\approx 1.0 \sigma_{c}$ near the nominal JES value.

\section{3 $M_{\text {top }}$ Measurement}

After the JES determined purely from $W \rightarrow j j$ has been checked to be compatible with the a priori determination, we proceed with the measurement of the top quark mass. The top quark mass is measured simultaneously with the JES to take into account the correlations between these two observables. The information on JES from the $W \rightarrow j j$ measurement and the a priori calibration are combined to minimize the JES uncertainty. 


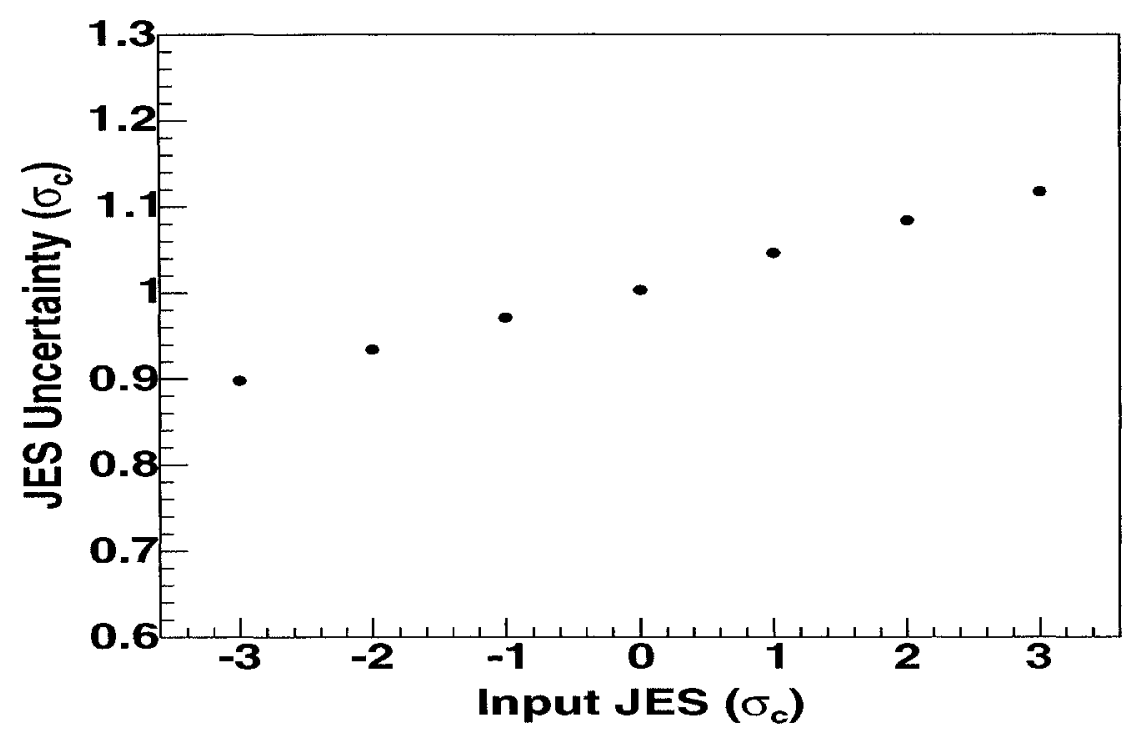

Figure 7.2: JES statistical uncertainty as a function of input JES for the JES cross-check.

\subsubsection{Description of the Likelihood Fit}

For each subsample, the likelihood function we maximize is

$$
\mathcal{L}_{\text {sample }_{i}}=\mathcal{L}_{\text {shape }}^{m_{t}} \times \mathcal{L}_{\text {shape }}^{m_{j j}} \times \mathcal{L}_{\text {nev }} \times \mathcal{L}_{\text {bg }}
$$

where,

$$
\begin{aligned}
& \mathcal{L}_{\text {shape }}^{m_{t}^{\text {reco }}}=\prod_{k=1}^{r^{W}} \frac{\epsilon_{s} n_{s}^{W} P_{s i g}\left(m_{k}^{t} ; M_{\text {top }}, J E S\right)+\epsilon_{b} n_{b}^{W} P_{b}\left(m_{k}^{t}\right)}{\epsilon_{s} n_{s}^{W}+\epsilon_{b} n_{b}^{W}} \\
& \mathcal{L}_{\text {shape }}^{m_{j j}}=\prod_{k=1}^{{ }^{W} \times n_{i}^{\text {comb }}} \frac{n_{s}^{W} P_{\text {sig }}\left(m_{k}^{j j} ; M_{\text {top }}, J E S\right)+n_{b}^{W} P_{b}\left(m_{k}^{j j}\right)}{n_{s}^{W}+n_{b}^{W}}, \\
& \mathcal{L}_{\text {nev }}=\sum_{r_{s}^{W}+r_{b}^{W}=r^{W}} P_{\text {Pois }}\left(r_{s}^{W} ; n_{s}^{W}\right) P_{\text {Pois }}\left(r_{b}^{W} ; n_{b}^{W}\right) \times \\
& {\left[\sum_{r_{s}^{t}+r_{b}^{t}=r^{t}}^{r_{s}^{t} \leq r_{s}^{W}, r_{b}^{t} \leq r_{b}^{W}} P_{\text {Binom }}\left(r_{s}^{t} ; r_{s}^{W}, \epsilon_{s}\right) P_{\text {Binom }}\left(r_{b}^{t} ; r_{b}^{W}, \epsilon_{b}\right)\right]} \\
& \mathcal{L}_{b g}=\exp \left(-\frac{\left(n_{b}^{W}-n_{b}^{W}(\text { constr })\right)^{2}}{2 \sigma_{n_{b}^{W}}^{2}}\right)
\end{aligned}
$$


The function is maximized with respect to the parameters $M_{t o p}, \mathrm{JES}, n_{s}^{W}$ and $n_{b}^{W}$. The term $\mathcal{L}_{\text {shape }}^{m_{t}^{\text {reco }}}\left(\mathcal{L}_{\text {shape }}^{m_{j j}}\right)$ contains the probability that a given reconstructed top quark mass $m_{k}^{t}$ (W boson mass $\left.m_{k}^{j j}\right)$ comes from signal given a true $M_{\text {top }}$ and JES $P_{\text {sig }}\left(m_{k}^{t} ; M_{\text {top }}, J E S\right)$ $\left(P_{s i g}\left(m_{k}^{j j} ; M_{t o p}, J E S\right)\right)$ or from background $P_{b}\left(m_{k}^{t}\right)\left(P_{b}\left(m_{k}^{j j}\right)\right)$. The functional form for both the $m_{t}^{r e c o}$ and $m_{j j}$ signal probabilities is given in Eqns. 5.8-5.9. The expression for

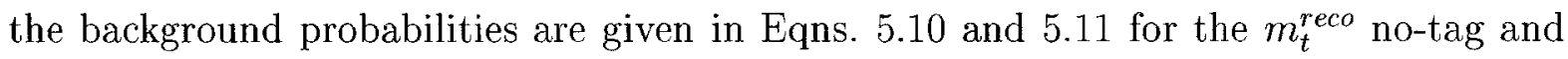
tagged subsamples, respectively, and Eqns. 6.1 and 6.2 for the $m_{j j}$ no-tag and tagged subsamples, respectively.

Only two parameters are used to model the expected number of signal and background events in the likelihood fit. Indeed, the number of events in the $m_{t}^{r e c o}$ and $m_{j j}$ samples are correlated and the only selection difference is the $\chi_{\min }^{2}$ cut that is applied to the $m_{t}^{\text {reco }}$ sample. Therefore, we choose to use for parameters the expected number of events in the $m_{j j}$ sample $n_{s}^{W}$ and $n_{b}^{W}$ for signal and background, respectively. The expected number of events in the $m_{t}^{r e c o}$ sample is then simply given by $\epsilon_{s} n_{s}^{W}$ and $\epsilon_{b} n_{b}^{W}$ where $\epsilon_{s}$ and $\epsilon_{b}$ are $\chi_{\min }^{2}$ cut efficiencies for the signal and background, respectively. The $\chi_{\min }^{2}$ cut efficiencies are given in Table 7.2. The background efficiencies are a combination of the efficiencies for each background source normalized by the expected number of events from each source. The $\chi_{\min }^{2}$ cut efficiencies are extracted at the nominal JES and using a Monte Carlo sample with $M_{t o p}=178 \mathrm{GeV} / c^{2}$ for the signal. ${ }^{2}$

The term $\mathcal{L}_{\text {nev }}$ expresses the probability to observe respectively $r^{W}$ and $r^{t}$ events in the $m_{j j}$ and $m_{t}^{r e c o}$ samples given the expected number of signal and background events in the $m_{j j}$ samples, $n_{s}^{W}$ and $n_{b}^{W}$, and the $\chi_{m i n}^{2}$ cut efficiencies, $\epsilon_{s}$ and $\epsilon_{b}$. The first sum expresses the Poisson probability to observe $r_{s}^{W}$ signal and $r_{b}^{W}$ background events given Poisson means of $n_{s}^{W}$ and $n_{b}^{W}$, respectively. The sum is on the number of observed signal

\footnotetext{
${ }^{2}$ The $\chi_{\min }^{2}$ efficiencies have some small dependence on $M_{\text {top }}$ and JES. We have attempted to parametrize and include this dependence in the likelihood, but only a negligible effect has been observed (less than $0.1 \mathrm{GeV} / c^{2}$ in $M_{t o p}$ ). We conclude that the use of constant $\chi_{\min }^{2}$ efficiencies in the likelihood is sufficient for our purpose.
} 


\begin{tabular}{ccc}
\hline \hline Subsample & $\epsilon_{s}$ & $\epsilon_{b}$ \\
\hline 2-tag & $65.1 \%$ & $37.6 \%$ \\
$1-\operatorname{tag}(\mathrm{T})$ & $85.4 \%$ & $73.5 \%$ \\
1-tag(L) & $79.6 \%$ & $71.3 \%$ \\
0-tag & $91.4 \%$ & $82.6 \%$ \\
\hline \hline
\end{tabular}

Table 7.2: The $\chi_{\min }^{2}$ cut efficiencies for signal and background extracted at nominal JES and $M_{\text {top }}=178 \mathrm{GeV} / c^{2}$ for signal.

and background events that equal the total number of observed events in the $m_{j j}$ sample, $r_{s}^{W}+r_{b}^{W}=r^{W}$. For each pair $\left(r_{s}^{W}, r_{b}^{W}\right)$, we calculate the binomial probability to observe $r_{s}^{t}$ signal and $r_{b}^{t}$ background events in the $m_{t}^{\text {reco }}$ sample given $r_{s}^{W}$ and $r_{b}^{W}$ and the $\chi_{\min }^{2}$ cut efficiencies $\epsilon_{s}$ and $\epsilon_{b}$. The sum is over the number of observed signal and background events in the $m_{t}^{\text {reco }}$ sample that equal the total number of observed events: $r_{s}^{t}+r_{b}^{t}=r^{t}$.

Similarly to the JES cross-check analysis, the number of background events in the $m_{j j}$ sample is constrained to the a priori expectation as expressed by $\mathcal{L}_{b g}$.

The final likelihood function is given by the product of the four subsample likelihood functions times the Gaussian constraint to the a priori calibration of JES $\left(\mathcal{L}_{J E S}\right)$

$$
\mathcal{L}_{\text {total }}=\mathcal{L}_{2-\operatorname{tag}} \times \mathcal{L}_{1-\operatorname{tag}(T)} \times \mathcal{L}_{1-\operatorname{tag}(L)} \times \mathcal{L}_{0-\operatorname{tag}} \times \mathcal{L}_{J E S}
$$

where

$$
\mathcal{L}_{J E S}=\exp \left(-\frac{\left(J E S-J E S^{\text {constr }}\right)^{2}}{2 \sigma_{J E S^{\text {constr }}}^{2}}\right) .
$$

Since the CDF calibration centers JES at $0 \sigma_{c}$ with a width of $1 \sigma_{c}$, Eqn. 7.13 simplifies to

$$
\mathcal{L}_{J E S}=\exp \left(-\frac{J E S^{2}}{2}\right) .
$$

The measured values of $M_{t o p}$ and JES are the ones that simultaneously maximize the final likelihood function. The same definition of the uncertainties used for the JES cross-check is employed for the $M_{t o p}$ measurement, i.e. a one standard deviation uncer- 
tainty corresponds to the value of $M_{t o p}(\mathrm{JES})$ where the logarithm of the likelihood has decreased by half a unit with respect to the most probable value (where the likelihood is re-optimized away from the maximum with respect to all other parameters: $n_{s}^{W}, n_{b}^{W}$ and JES $\left.\left(M_{t o p}\right)\right)$. The uncertainty on the background normalization is reflected on the $M_{t o p}$ and JES uncertainties. Again these uncertainties need to be corrected by scale factors extracted in Sec. 7.3.3 to ensure adequate statistical coverage of the $1 \sigma$ interval.

\subsubsection{Pseudo-Experiments Procedure}

A pseudo-experiment procedure is used to assess the performance of the $M_{\text {top }}$ likelihood fit and to estimate systematic uncertainties. The procedure is very similar to the one used for the JES cross-check:

1. The number of signal and background events in the $m_{j j}$ sample for each experiment are generated randomly from Poisson distributions as described in Sec. 7.2.2. The number of events in the $m_{t}^{r e c o}$ samples is drawn from a binomial distribution of the number of events in the $m_{j j}$ samples generated previously assuming a binomial probability that corresponds to the $\chi_{\min }^{2}$ efficiencies given in Table 7.2. This is done for signal and background events separately.

2. The reconstructed masses $m_{t}^{r e c o}$ and $m_{j j}$ are generated randomly from the corresponding histograms for a given input $M_{t o p}$ and JES. The correlations between the various $m_{j j}$ 's and $m_{t}^{r e c o}$ in the same event are ignored (see Sec. 7.3.4 on that subject). The number of masses generated in each sample is given by step 1 .

3. The central value of the Gaussian constraint terms $\mathcal{L}_{b g}$ and $\mathcal{L}_{J E S}$ are randomly drawn from Gaussian distributions with the corresponding mean and width. As mentioned before, this is the correct way in a constrained fit to obtain normally distributed pull distributions [53].

4. The maximization of the $M_{\text {top }}$ measurement likelihood function is performed. 
Typically a few thousand of these pseudo-experiments are generated to test the performance of the likelihood fit.

\subsubsection{Assessment of the Likelihood Performance}

We check that the central values and uncertainties of $M_{\text {top }}$ and JES returned by the $M_{\text {top }}$ measurement likelihood fit are unbiased by performing pseudo-experiments for large ranges of input $M_{\text {top }}$ and JES.

We compute the pull distributions of the likelihood fit (defined for the JES cross-check in Sec. 7.2.3). The means of a Gaussian fit to the $M_{\text {top }}$ pull distributions are shown in Fig. 7.3 as a function of $M_{t o p}$ (for JES $=0 \sigma_{c}$ ) and JES (for $M_{t o p}=175 \mathrm{GeV} / c^{2}$ ). Similar plots are shown in Fig. 7.4 for the JES pull distributions. No significant bias in terms of the fitted $M_{\text {top }}$ and JES are observed for large range of input $M_{\text {top }}$ and JES apart from a small negative bias in the $M_{\text {top }}$ pull mean of $-0.07 \pm 0.02$ (see left plot of Fig. 7.3), corresponding to a bias of approximately $-0.3 \pm 0.1 \mathrm{GeV} / c^{2}$ in terms of $M_{\text {top }}$. This value is included in the method systematic uncertainty described in Sec. 8.2. A small negative bias can be observed on the right plot of Fig. 7.4 but it is consistent with a statistical fluctuation for the point $M_{t o p}=175 \mathrm{GeV} / c^{2}$ on the left plot. ${ }^{3}$

We check that the $M_{t o p}$ and JES uncertainties returned by the fit procedure are unbiased by looking at the width of the pull distributions (defined as the width of a Gaussian fit to the pull distributions). The $M_{t o p}$ pull widths are shown in Fig. 7.5 as a function of input $M_{t o p}$ (for JES $=0 \sigma_{c}$ ) and JES (for $M_{t o p}=175 \mathrm{GeV} / c^{2}$ ). A fit of these distributions to a constant suggest the uncertainties need to be inflated by $1.027 \pm 0.006$ (versus $M_{t o p}$ ) or $1.031 \pm 0.006$ (versus JES). We scale the $M_{\text {top }}$ uncertainty returned by the fit by the former scale factor (1.027) since the parameter of interest in the fit is $M_{\text {top }}$. The JES pull widths are shown in Fig. 7.6 as a function of input $M_{t o p}$ (for JES $=0 \sigma_{c}$ )

\footnotetext{
${ }^{3}$ The pull distributions for various JES at a given input $M_{\text {top }}$ are correlated since they are generated from the same MC sample.
} 

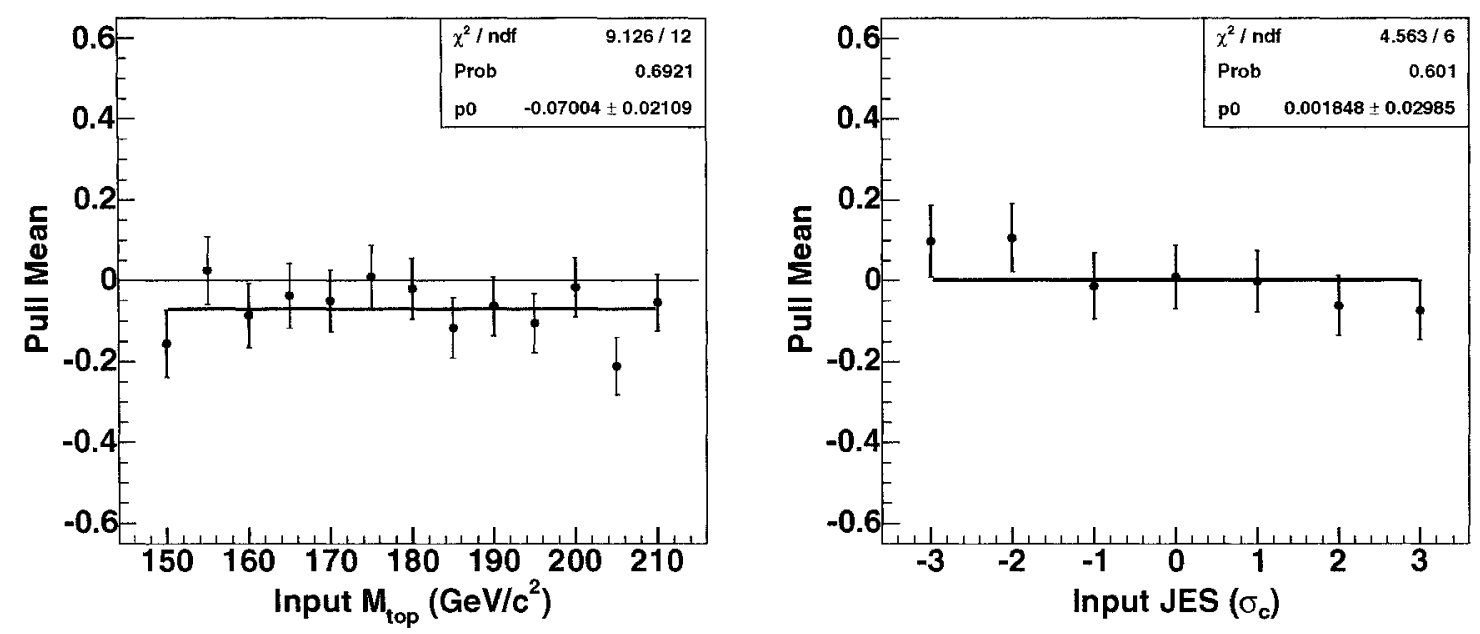

Figure 7.3: Mean of the $M_{t o p}$ pull distributions as a function of the input $M_{t o p}$ (at nominal JES; left plot) and the input JES $\left(M_{t o p}=175 \mathrm{GeV} / c^{2}\right.$; right plot $)$ for the $M_{t o p}$ measurement.

and JES (for $M_{t o p}=175 \mathrm{GeV} / c^{2}$ ). The JES uncertainties need to be inflated by the modest factor of $1.014 \pm 0.005$.

The median of the $M_{\text {top }}$ uncertainty extracted from the likelihood fit (scaled by the factor 1.027 determined above) is shown in Fig. 7.7 as a function of $M_{t o p}$ (for JES $=0$ $\sigma_{c}$ ) and JES (for $M_{t o p}=175 \mathrm{GeV} / c^{2}$ ). The expected uncertainty for $M_{t o p}=178 \mathrm{GeV} / c^{2}$ and JES $=0 \sigma_{c}$ is $\approx 4.3 \mathrm{GeV} / c^{2}$ for the current dataset (this includes both the statistical and JES uncertainties). The expected weight of each subsample in the combined $M_{\text {top }}$ uncertainty is $35.4 \%, 40.5 \%, 11.5 \%$ and $12.5 \%$ for the 2 -tag, $1-\operatorname{tag}(\mathrm{T}), 1-\operatorname{tag}(\mathrm{L})$ and 0 -tag subsamples, respectively. The traditional template method [51] is expected to yield an uncertainty of $\approx 4.8 \mathrm{GeV} / c^{2}$, including both the statistical and JES uncertainties. The $M_{\text {top }}$ measurement thus provide an improvement of about $10 \%$ in the $M_{\text {top }}$ uncertainties with respect to the traditional template method (i.e. without the $W \rightarrow j j$ measurement) with the current dataset. We note that the gap in terms of statistical+JES uncertainties between these two analyses is expected to increase as more data is accumulated since the 

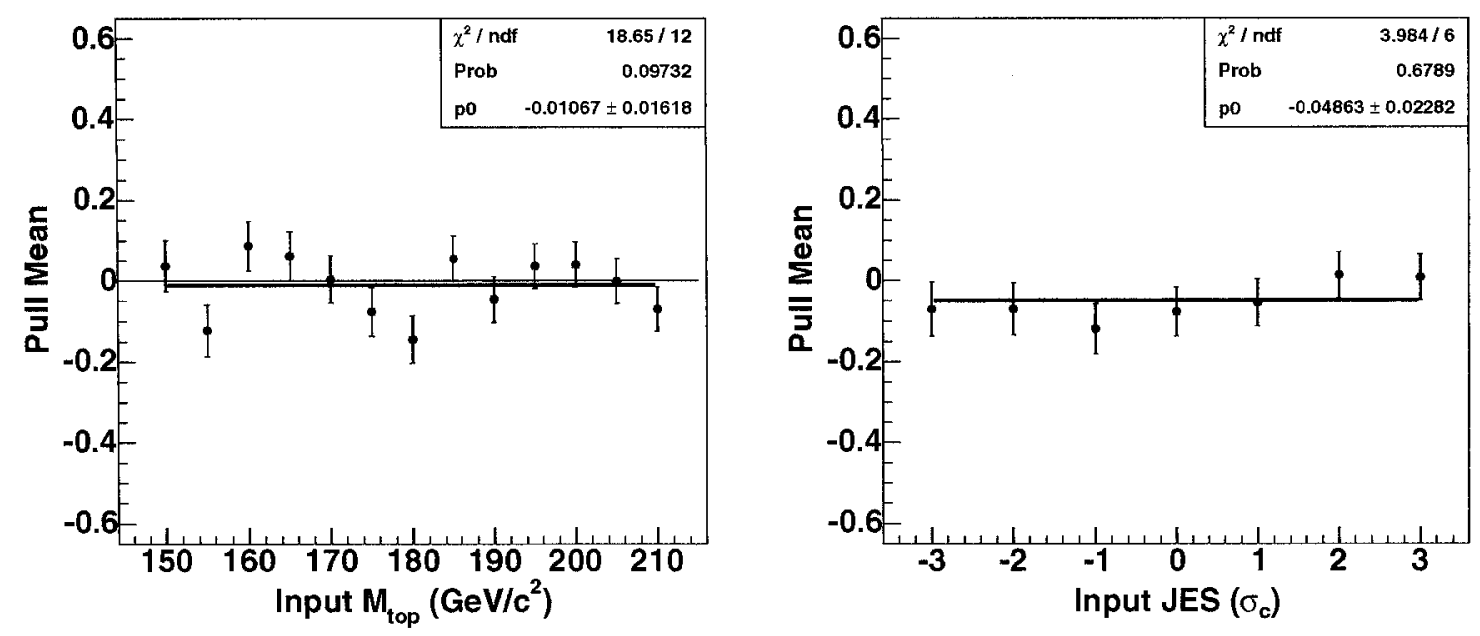

Figure 7.4: Mean of the JES pull distributions as a function of the input $M_{t o p}$ (at nominal JES; left plot) and the input JES $\left(M_{t o p}=175 \mathrm{GeV} / c^{2}\right.$; right plot) for the $M_{t o p}$ measurement. An overall negative bias is observed on the right plot that is consistent with a statistical fluctuation for the point $M_{t o p}=175 \mathrm{GeV} / c^{2}$ on the left plot.

JES uncertainty from the $W \rightarrow j j$ measurement will further improve. ${ }^{4}$

The median of the JES uncertainty is shown in Fig. 7.8 as a function of $M_{t o p}$ (for JES $=0 \sigma_{c}$ ) and JES (for $M_{t o p}=175 \mathrm{GeV} / c^{2}$ ). The expected JES uncertainty is $\approx 0.76 \sigma_{c}$ for $M_{t o p}=178 \mathrm{GeV} / c^{2}$ and JES $=0 \sigma_{c}$. We thus conclude that the combination of the $a$ priori calibration and the $W \rightarrow j j$ measurement provides an improvement of $24 \%$ of the JES uncertainty (with respect to using the a priori jet energy calibration alone). This is in agreement with the combination of two measurements with equal uncertainties of $1 \sigma_{c}$ (since both measurements have expected uncertainties of $1 \sigma_{c}$ with the current dataset).

\subsubsection{Further Checks}

Before applying the likelihood fit to the data, additional studies of the fitting procedures have been performed.

${ }^{4}$ This aspect of the technique is discussed in more detail in Chapter 10. 

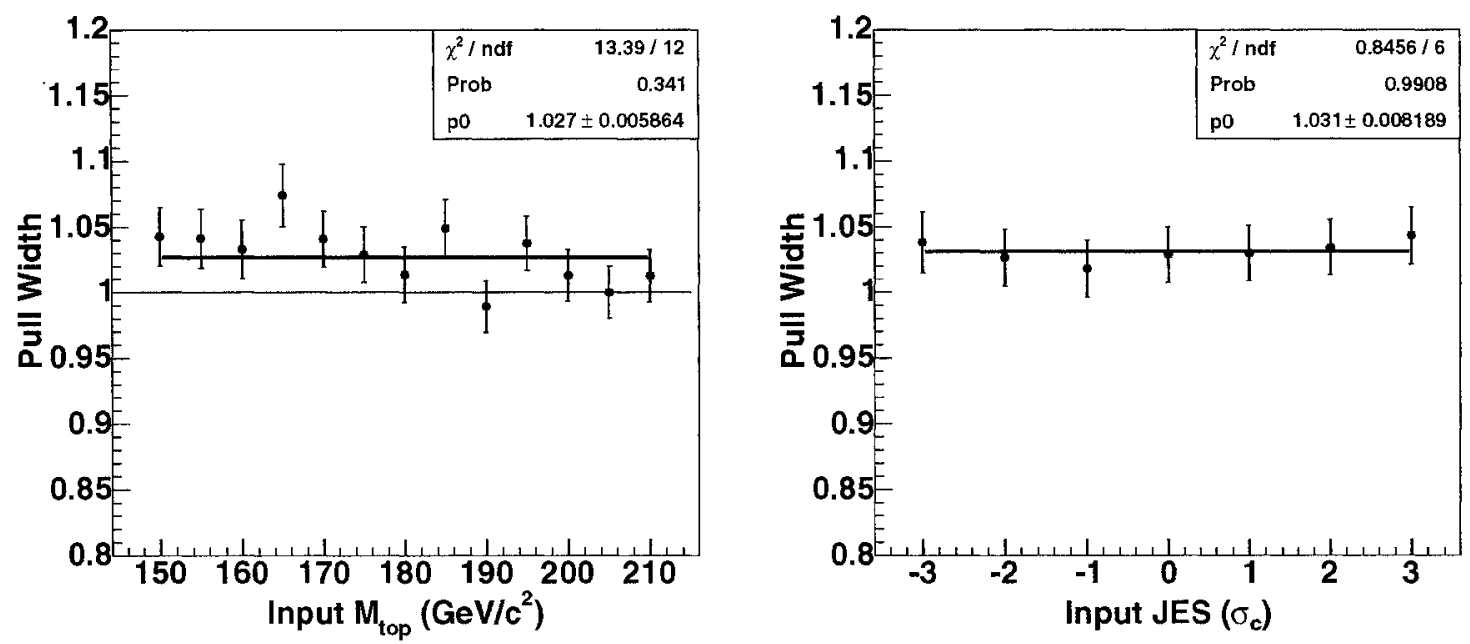

Figure 7.5: Width of the $M_{\text {top }}$ pull distributions as a function of the input $M_{t o p}$ (at nominal JES; left plot) and the input JES $\left(M_{t o p}=175 \mathrm{GeV} / c^{2}\right.$; right plot) for the $M_{t o p}$ measurement.

- Correlations between $m_{j j}$ and $m_{t}^{r e c o}$ : The fitting procedure treats $m_{t}^{r e c o}$ and the various $m_{j j}$ in the same event as uncorrelated. This is not expected to be the case in reality since they are partially reconstructed from the same objects. Therefore, we have studied the impact of neglecting these correlations in the likelihood fit and the pseudo-experiments procedure.

We first investigate the correlations between $m_{t}^{r e c o}$ and $m_{j j}$ in the same event. Twodimensional plots of $m_{t}^{r e c o}$ versus each of the three $m_{j j}$ combinations for the $1-\operatorname{tag}(\mathrm{T})$ subsample are given in Fig. 7.9 for a signal sample with $M_{t o p}=178 \mathrm{GeV} / c^{2}$. We observe no striking correlations between $m_{t}^{r e c o}$ and $m_{j j}$ from these plots. Still, the calculated correlation factors are non-zero (typically 0.2 ), and thus prevent us from concluding that the effect is negligible.

A more systematic study is performed by generating pseudo-experiments where the correlations between $m_{t}^{\text {reco }}$ and $m_{j j}$ and the various $m_{j j}$ combinations in an event are simulated for the signal sample. This is done by generating the pseudo- 

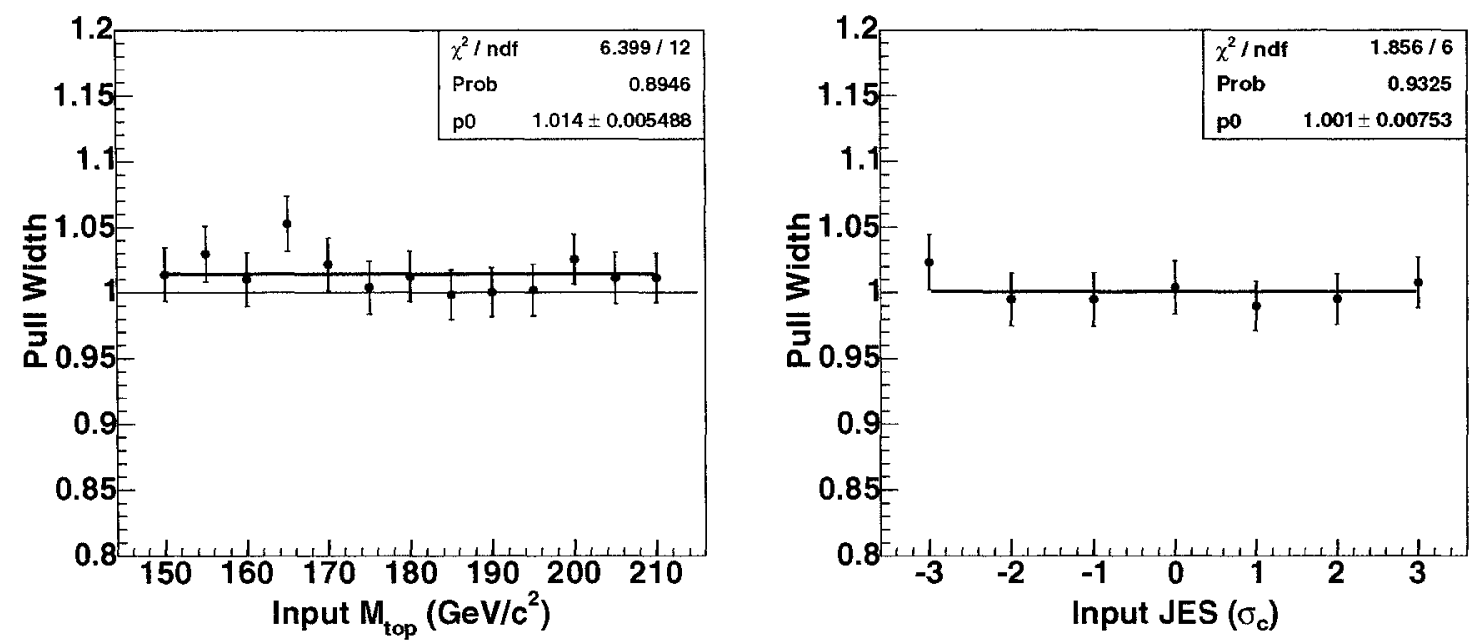

Figure 7.6: Width of the JES pull distributions as a function of the input $M_{t o p}$ (at nominal JES; left plot) and the input JES $\left(M_{\text {top }}=175 \mathrm{GeV} / c^{2}\right.$; right plot) for the $M_{t o p}$ measurement.

experiments from fully reconstructed $t \bar{t}$ events. The mean and width of the $M_{\text {top }}$ pull distributions are shown in Fig. 7.10 as a function of the input $M_{t o p}$ for the pseudoexperiments with mass correlations simulated. We obtain results consistent with the ones obtained with the default procedure (see Figs. 7.3 and 7.5). We conclude that the $M_{\text {top }}$ measurement is not biased by neglecting the mass correlations in the likelihood fit or the pseudo-experiments. We note that the same conclusion holds for the fitted JES (not shown here).

- Jet energy resolution: In principle, the top quark mass measurement could be sensitive to discrepancies in the resolution of the jet energy response between data and $\mathrm{MC}$ events. Indeed, the jet energy response is dependent on the jet energy resolution as discussed in the case of the top-specific corrections in Sec. 5.2.2.

The jet resolution can be estimated from photon+jet events where the photon energy resolution is small compared to that of the jet. The jet energy resolution in this type of events is measured to be $17.9 \pm 0.2 \%$ for data and $17.5 \pm 0.2 \%$ for HER- 

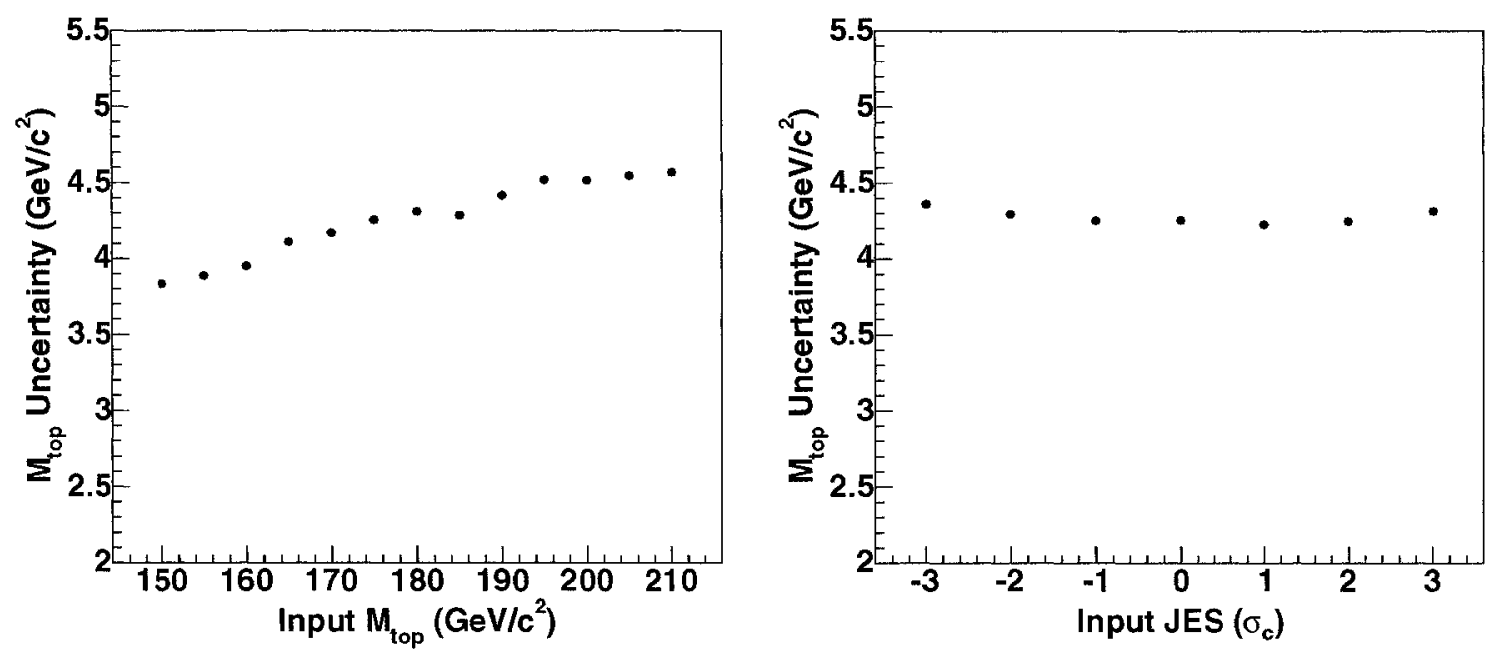

Figure 7.7: Median of the expected $M_{t o p}$ uncertainties as a function of the input $M_{t o p}$ (at nominal JES; left plot) and the input JES $\left(M_{t o p}=175 \mathrm{GeV} / c^{2}\right.$; right plot) for the $M_{\text {top }}$ measurement for $\int \mathcal{L} d t=318 \mathrm{pb}^{-1}$.

WIG MC events. This correspond to a jet energy resolution discrepancy between data and $\mathrm{MC}$ of $4 \%$ (difference in quadrature). We have smeared the jets by this amount in HERWIG $t \bar{t}$ events and estimated the impact on the fitted top quark mass using pseudo-experiments. We find that the sample with smeared jets yields $M_{\text {top }}$ values that are different by less than $0.05 \mathrm{GeV} / c^{2}$. Therefore, we conclude that the impact of any uncertainty in the jet energy resolution is negligible in this analysis. 

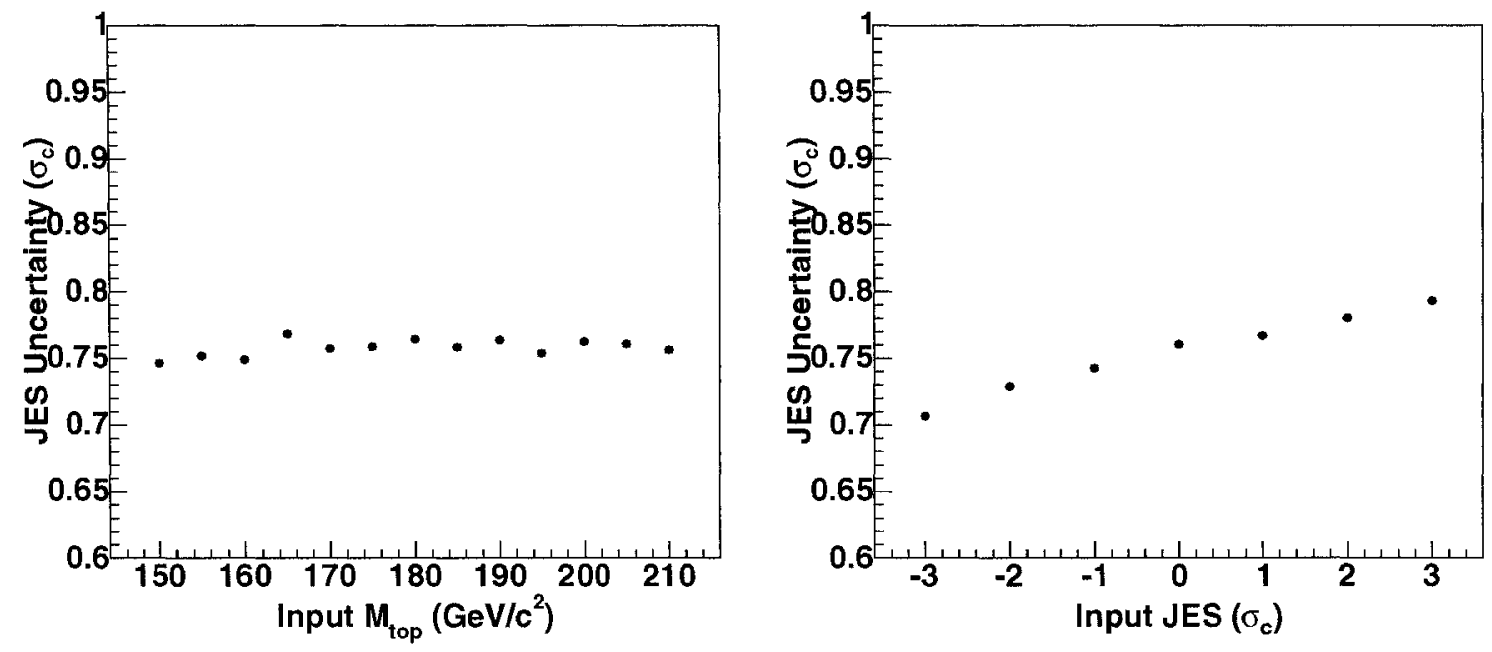

Figure 7.8: Median of the expected JES uncertainties as a function of the input $M_{\text {top }}$ (at nominal JES; left plot) and the input JES $\left(M_{t o p}=175 \mathrm{GeV} / c^{2} ;\right.$ right plot $)$ for the $M_{t o p}$ measurement for $\int \mathcal{L} d t=318 \mathrm{pb}^{-1}$.
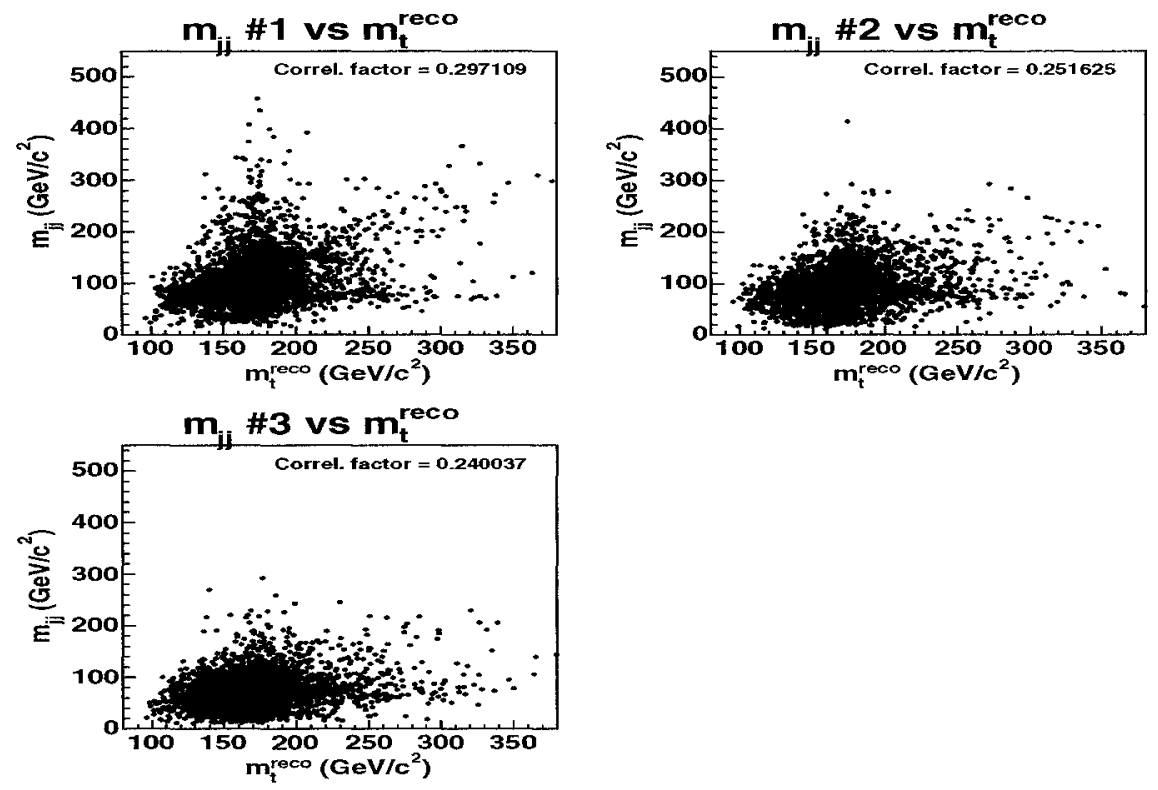

Figure 7.9: The three $m_{j j}$ combinations (ordered in decreasing value of $m_{j j}$ ) in the 1-tag(T) subsample versus $m_{t}^{r e c o}$ for a signal sample with $M_{t o p}=178 \mathrm{GeV} / c^{2}$. 

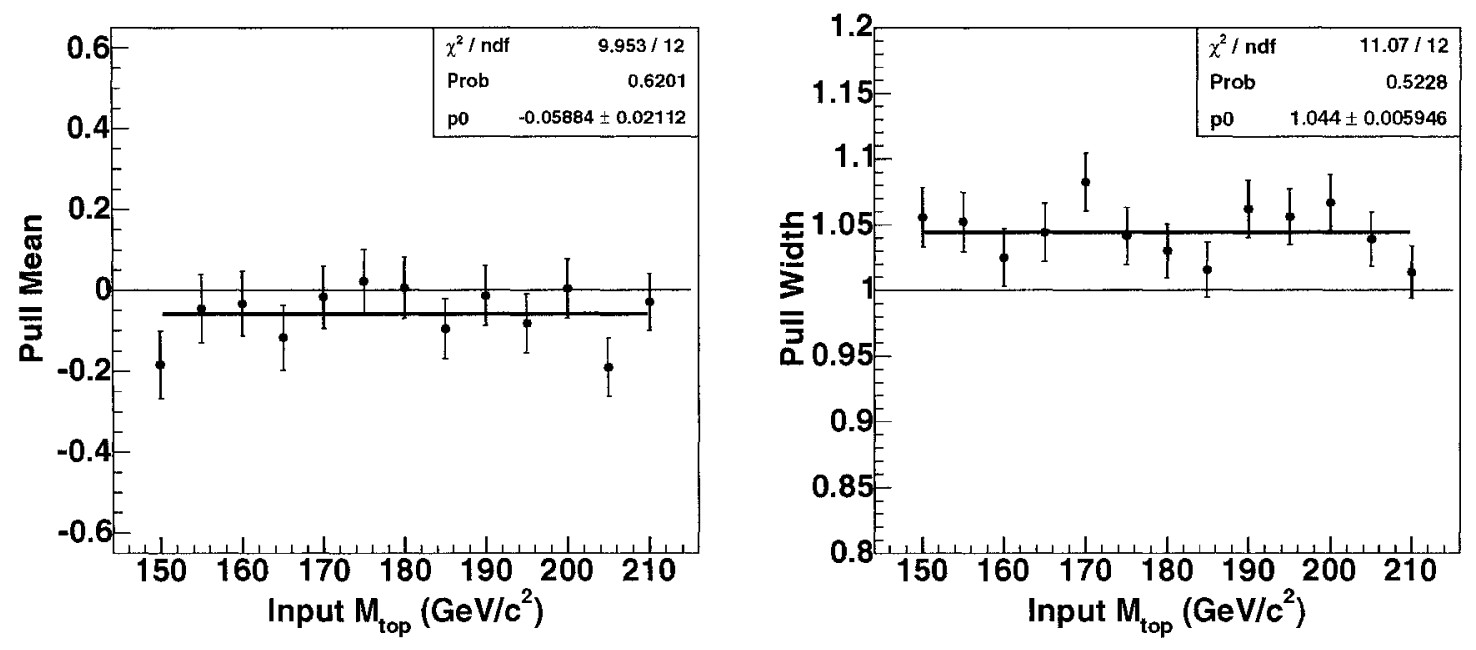

Figure 7.10: Mean (left plot) and width (right plot) of the $M_{t o p}$ pull distribution as a function of the input $M_{t o p}$ for the $M_{t o p}$ measurement where the correlations between the reconstructed masses in each event are simulated. 


\section{Chapter 8}

\section{Systematic Uncertainties}

The uncertainties returned by the likelihood fits are only due to the finite statistics of the sample and the allowed variations of JES. Since the top quark mass is measured by comparing real data with simulated events, systematic uncertainties in the measurement arise from the MC generation of signal and background events and the simulation of the CDF detector. In this chapter we describe the sources of systematic uncertainties and their effect on the fitted $M_{\text {top }}$ and JES.

The systematics uncertainties are estimated using the pseudo-experiment procedures described in Chapter 7 where the simulated data are generated from a signal or background reconstructed mass shape to which a systematic effect is applied (e.g. the use of a different set of parton distribution functions) and the shifts in median fitted $M_{t o p}$ and JES are used to estimate the size of the systematic uncertainty associated with the effect.

\subsection{B-Jets Energy Scale}

The CDF jet energy calibration or $W \rightarrow j j$ measurement of JES do not give direct information on the $b$-jets energy scale. This is a critical omission as $b$-jets come directly from the top quark decays and thus affect more significantly the top quark mass than 
the $W$ daughter jets. Furthermore, the $W$ daughter jets are constrained to the known $W$ mass in the $\chi^{2}$ fit, thereby reducing their JES dependence, whereas $b$-jets have no such constraints. Therefore, any additional uncertainties arising from the specificities of $b$-jets must be taken into account.

We have identified the following differences between $b$-jets and $W$ daughter jets that may affect the jet energy scale:

- Heavy quark fragmentation: The $b$-hadron resulting from the $b$-quark fragmentation carries a larger fraction of the parent quark momentum than for light quark fragmentation. This is because $b$-quarks are much heavier and thus only slightly decelerated when combined with a light quark to form a $b$-hadron.

- Colour flow: $W$ daughter jets come from the decay of a colour singlet, while $b$-jets have a colour connection with the initial state partons inside the protons.

- Decay: A large fraction of the decay products of $b$ or $c$ hadrons are charged leptons and neutrinos from semileptonic decays. These particles interact very differently with the calorimeter than the more common hadronic particles. Therefore, $b$-jets have a different response on average than $W$ daughter jets because of the larger semileptonic decay fraction. ${ }^{1}$ These decays are modeled by the MC that is used to extract the top-specific energy corrections, but the uncertainty arising from the limited precision of the measurements of semi-inclusive leptonic branching fractions have to be taken into account.

We consider that the jet energy scale uncertainties determined by the CDF jet energy calibration or $W \rightarrow j j$ measurements of JES are common to $b$-jets, generic and light quark jets. Since the $b$-jets characteristics above are not constrained by these calibrations, we calculate separately their uncertainties as follows.

\footnotetext{
${ }^{1}$ About $35 \%$ of $b$-jets contain the semileptonic decay of a $b$ or $c$ quark hadron.
} 
The heavy quark fragmentation uncertainty is estimated using experimental constraints obtained by the LEP and SLD experiments from the study of $Z \rightarrow b \bar{b}$ decays [54]. We generate PYTHIA samples of $t \bar{t}$ events with variations of the Peterson fragmentation parameter [55] that are representative of the experimental constraints on this parameter. These samples are also compared with the Bowler heavy quark fragmentation function [56] that is used by default in PYTHIA. Pseudo-experiments are generated from these samples and a maximal deviation of $0.2 \pm 0.4 \mathrm{GeV} / c^{2}$ in the fitted $M_{\text {top }}$ is observed. We use the uncertainty on the estimate $\left(0.4 \mathrm{GeV} / c^{2}\right)$ as the uncertainty on heavy quark fragmentation. This corresponds to an uncertainty of $0.13 \sigma_{c}$ on JES.

The uncertainty from $b$-jets semileptonic decays is extracted by varying the inclusive semileptonic branching ratio within its uncertainty [5] and estimating the impact on the jet energy scale. The resulting variation on the jet energy scale is estimated to be $0.4 \%$ or $0.13 \sigma_{c}$, which corresponds to an uncertainty on $M_{t o p}$ of $0.4 \mathrm{GeV} / c^{2}$.

We also check the induced shifts in JES due to variations of the modeling of the colour flow in $t \bar{t}$ events. The amount of "ambiguous energy", i.e. energy that cannot be unambiguously assigned to come from the $b$-jet or the initial state parton due to their colour connection has been estimated to represent $3 \%$ of the total $b$-jets energy scale in HERWIG MC events. By considering large variations of the parameter related to the colour flow modeling, this amount of ambiguous energy has shifted by $10 \%$ relatively, or $0.3 \%$ of the total $b$-jets energy. Therefore, we assign an uncertainty of $0.3 \%\left(0.10 \sigma_{c}\right)$ on the JES that corresponds to $0.3 \mathrm{GeV} / c^{2}$ on $M_{\text {top }}$ due to the modeling of the colour flow of $b$-jets.

The $b$-jets energy scale uncertainties are summarized in Table 8.1. Adding these in quadrature, we get a total uncertainty of $0.6 \mathrm{GeV} / c^{2}$ on the top quark mass $\left(0.25 \sigma_{c}\right.$ on JES) due to the specificities of $b$-jets. We note that these uncertainties do not affect the JES cross-check that only attempts to check the JES of $W$ daughter jets between data and MC. More detail on the estimation of the $b$-jets uncertainties can be found in [57]. 


\begin{tabular}{lcc}
\hline \hline Source & $\Delta M_{\text {top }}\left(\mathrm{GeV} / c^{2}\right)$ & $\Delta \mathrm{JES}\left(\sigma_{c}\right)$ \\
\hline Heavy quark fragmentation & 0.4 & 0.13 \\
Semileptonic decay & 0.4 & 0.13 \\
$b$-jets colour flow & 0.3 & 0.10 \\
\hline Total & 0.6 & 0.25 \\
\hline \hline
\end{tabular}

Table 8.1: Uncertainty from the specific characteristics of $b$-jets.

\subsection{Method Uncertainty}

The $m_{t}^{r e c o}$ and $m_{j j}$ templates are created as a function of JES by multiplying every jet by a common jet energy scale factor. This is an approximation since the discrepancy between the data and MC jet energy response can be different on a jet-by-jet basis. For example, one can imagine that low- $p_{T}$ jets have a JES that is $+2 \sigma_{c}$ higher in MC with respect to the data, but concurrently have a very good agreement for high- $p_{T}$ jets. This may affect the JES determination from $W \rightarrow j j$ decays that only measures the average JES discrepancy for $W$ daughter jets between data and MC.

We consider the three largest components of the JES systematic uncertainties of the traditional estimate (see Sec. 5.4), i.e. the ones associated with the relative response, the absolute scale and the out-of-cone energy. Each uncertainty has a different $p_{T}$ - and $\eta$-dependent shape. We have varied each of these components independently by their $\pm 1 \sigma$ variations for a total of 27 different variations of the JES discrepancy between data and MC. We have then performed pseudo-experiments based on these variations of the JES and find that the fitted value of $M_{t o p}$ is shifted on average by $0.4 \mathrm{GeV} / c^{2}$, which we take as the systematic uncertainty on $M_{\text {top }}$. Using the same method, we assign an uncertainty of $0.02 \sigma_{c}$ and $0.04 \sigma_{c}$ on the fitted JES for the $M_{\text {top }}$ measurement and JES cross-check, respectively.

We check the estimate of this uncertainty by introducing arbitrary $p_{T}$-dependent variations of the input JES. One example is illustrated in Fig. 8.1 where the JES is high by 


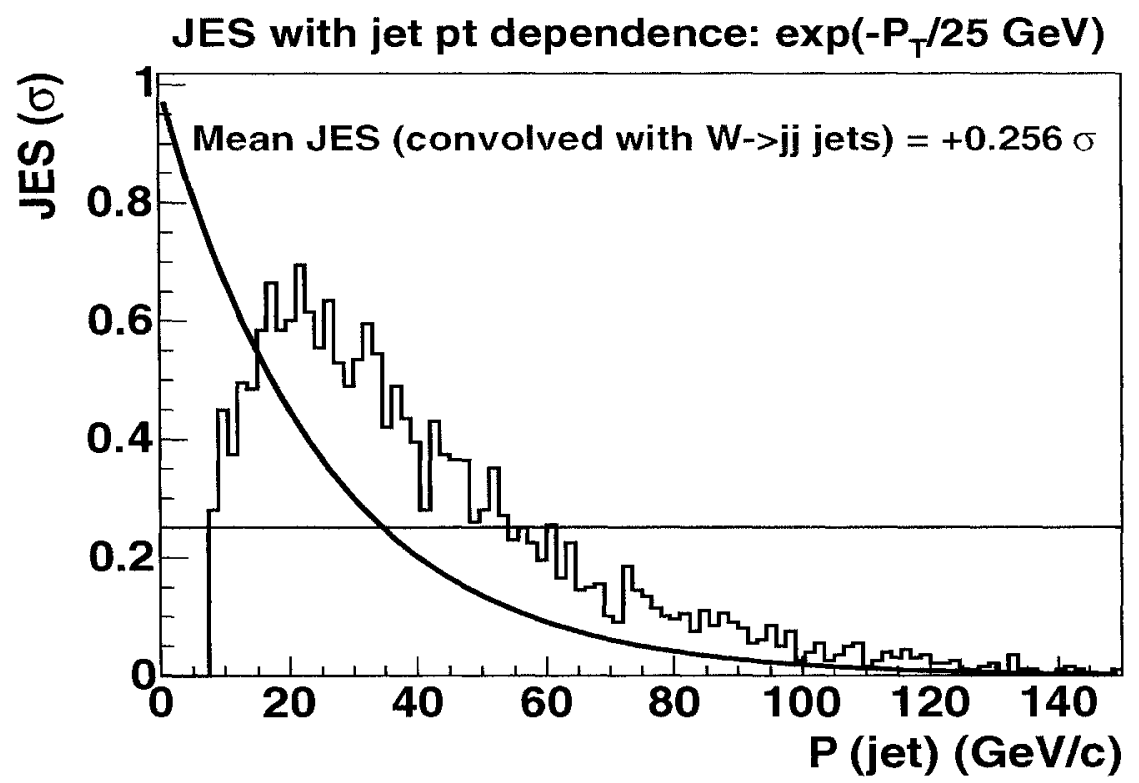

Figure 8.1: Solid curve: an example of a $p_{T}$-dependent variation of the JES discrepancy between data and $\mathrm{MC}$. The raw $p_{T}$ spectrum of $W$ daughter jets is overlaid as an histogram.

$\approx+1 \sigma_{c}$ at low- $p_{T}$ and asymptotically approaches $0 \sigma_{c}$ at large $p_{T}$. The raw $p_{T}$ spectrum of $W$ daughter jets is overlaid on Fig. 8.1. The convolution of the introduced JES $p_{T^{-}}$ dependence with the $W$ daughter jets $p_{T}$ distribution yields an average JES scale of +0.25 $\sigma_{c}$. Pseudo-experiments have been generated with this variation of JES for a sample with $M_{t o p}=178 \mathrm{GeV} / c^{2}$ and the mean of the fitted JES distribution is $+0.19 \pm 0.02 \sigma_{c}$, which is close to the average value above. The Gaussian mean of the fitted top quark mass is $177.2 \pm 0.2 \mathrm{GeV} / c^{2}$ that corresponds to a bias of $-0.8 \pm 0.2 \mathrm{GeV} / c^{2}$ for that particular example. More forms of the input JES have been attempted with similar size of the $p_{T}$-dependence and the average bias has been determined to be $0.5 \pm 0.1 \mathrm{GeV} / c^{2}$. This is in very good agreement with our estimate of the systematic uncertainty.

We also include as an additional uncertainty the average negative bias of $0.3 \mathrm{GeV} / c^{2}$ observed in the pull mean distribution of the top quark mass shown in Fig. 7.3. We add the two uncertainties in quadrature to get a total method uncertainty of $0.5 \mathrm{GeV} / c^{2}$. 


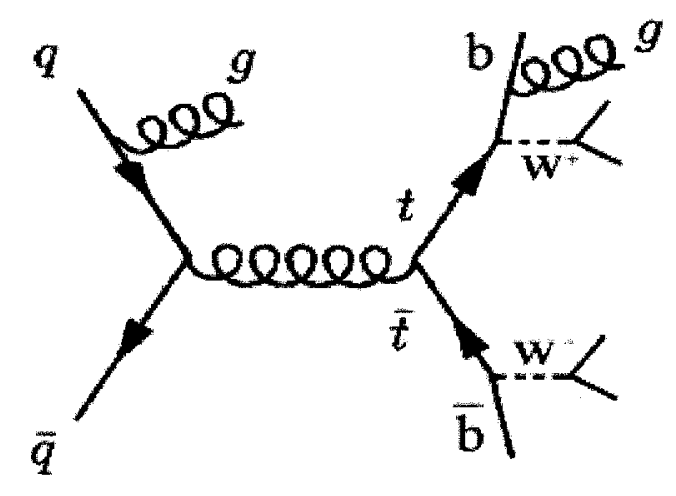

Figure 8.2: A tree level diagram of a $t \bar{t}$ event where a gluon is radiated from the incoming top quark (ISR) and $b$ quark propagators (FSR).

\subsection{Hard QCD Radiation}

As described in Chapters 5 and 6 , the top quark and $W$ boson mass reconstruction only considers the four leading jets that we assume originate from the four quarks of the $t \bar{t}$ decay chain. In reality a large fraction of $t \bar{t}$ events contain hard radiated gluons that result in one of the four highest $E_{T}$ jets. These events are in part responsible for the tails of the $m_{t}^{r e c o}$ and $m_{j j}$ templates as described in Chapters 5 and 6 . Gluons can be radiated from the initial state (ISR) or the final state (FSR) as illustrated in the Feynman diagram of Fig. 8.2. Any bias in the number of jets resulting from hard QCD radiation in MC $t \bar{t}$ events will affect the template shape and thus bias the top quark mass measurement.

Constraints on the modeling of ISR in $t \bar{t}$ is obtained by studying the $p_{T}$-spectrum of real data Drell-Yan $\left(Z / \gamma^{*}\right)$ events decaying to leptons and thus free of final state QCD radiation and by extrapolating the result to the $Q^{2}$ scale of $t \bar{t}$ production [58]. PYTHIA samples of $t \bar{t}$ events have been generated with "more" and "less" ISR corresponding to the findings of the Drell-Yan events study. The fitted top quark mass differences between the samples with more ISR and less ISR and the default PYTHIA sample are found to be $+0.43 \pm 0.25 \mathrm{GeV} / c^{2}$ and $+0.06 \pm 0.25 \mathrm{GeV} / c^{2}$, respectively. We use $0.4 \mathrm{GeV} / c^{2}$ as the systematic uncertainty due to ISR. Table 8.2 summarizes the observed shift in $M_{\text {top }}$ and 


\begin{tabular}{cccc}
\hline \hline Systematic effect & \multicolumn{2}{c}{$M_{\text {top }}$ Measurement } & JES Cross-Check \\
& $\Delta M_{\text {top }}\left(\mathrm{GeV} / c^{2}\right)$ & $\Delta J E S\left(\sigma_{c}\right)$ & $\Delta J E S\left(\sigma_{c}\right)$ \\
\hline more ISR - default PYTHIA & $+0.43 \pm 0.25$ & $+0.084 \pm 0.033$ & $+0.243 \pm 0.034$ \\
less ISR - default PYTHIA & $+0.06 \pm 0.25$ & $+0.043 \pm 0.033$ & $+0.064 \pm 0.034$ \\
\hline more FSR - default PYTHIA & $+0.24 \pm 0.36$ & $+0.063 \pm 0.046$ & $+0.238 \pm 0.047$ \\
less FSR - default PYTHIA & $+0.64 \pm 0.25$ & $+0.023 \pm 0.036$ & $+0.149 \pm 0.038$ \\
\hline PYTHIA - HERWIG & $-0.22 \pm 0.23$ & $-0.154 \pm 0.031$ & $-0.375 \pm 0.033$ \\
\hline \hline
\end{tabular}

Table 8.2: Shift in $M_{\text {top }}$ and JES due to variations of the ISR, FSR and MC generator.

JES for the $M_{\text {top }}$ measurement and the JES cross-check. We take the largest shifts as the systematic uncertainty on JES, i.e. $0.08 \sigma_{c}$ and $0.24 \sigma_{c}$ for the $M_{\text {top }}$ measurement and JES cross-check, respectively.

Since gluon radiation from the final state partons is equivalent to radiation from the initial state partons from a theoretical point of view, the Drell-Yan events study mentioned above can be used to provide constraints on FSR. Samples of PYTHIA $t \bar{t}$ events are generated with "more" and "less" FSR. The shifts observed in $M_{t o p}$ and JES are given in Table 8.2. We take the largest shifts observed as the uncertainties, i.e. $0.6 \mathrm{GeV} / c^{2}$ on $M_{\text {top }}$ and $0.06 \sigma_{c}$ and $0.24 \sigma_{c}$ on the JES in the $M_{\text {top }}$ measurement and JES cross-check, respectively.

\subsection{Generator}

The templates of $m_{t}^{r e c o}$ and $m_{j j}$ are extracted from $t \bar{t}$ samples generated with the HERWIG generator. We consider a systematic uncertainty from the general MC modeling by estimating the differences in fitted $M_{t o p}$ and JES between $t \bar{t}$ events generated with the HERWIG and PYTHIA Monte Carlo generator. The shifts observed are given in Table 8.2. We assume a generator systematic uncertainty of $0.2 \mathrm{GeV} / c^{2}$ on $M_{\text {top }}$ and 0.15 
$\sigma_{c}$ and $0.38 \sigma_{c}$ on the JES in the $M_{\text {top }}$ measurement and JES cross-check, respectively.

\subsection{Parton Distribution Functions}

Variations in the parton distribution functions (PDFs) can affect the kinematics of $t \bar{t}$ events and thus bias the top quark mass measurement. We estimate the uncertainty from PDFs by using a large HERWIG $t \bar{t}$ sample with $M_{\text {top }}=178 \mathrm{GeV} / c^{2}$, where the generated events are weighted according to their probability to occur according to different sets of PDFs. Several PDFs have been attempted:

- 0- CTEQ5L: default for the HERWIG $t \bar{t}$ samples used in this analysis

- 1- MRST72: Similar to CTEQ5L but from a different group

- 2- MRST75: Same as MRST72 but with the QCD scale parameter $\Lambda_{Q C D}=300$ $\mathrm{MeV}\left(\Lambda_{Q C D}=228 \mathrm{MeV}\right.$ for the default MRST72)

- 3- CTEQ6L: More recent fit from the CTEQ group than CTEQ5L

- 4- CTEQ6L1: Updated version with respect to CTEQ6L

- 5- CTEQ6M: Next-to-leading order PDFs from the CTEQ group

- 6-45- The PDFs uncertainties arising from the experimental uncertainties of the data used to obtain the PDFs are encoded under the form of 20 different uncertainties that can be varied by $\pm 1 \sigma$ resulting in 40 possible variations of the CTEQ6M PDFs.

The fitted $M_{t o p}$ as a function of the number of the PDFs set given above are shown in Fig. 8.3. The typical variations are small $\left(\mathcal{O}\left(0.1 \mathrm{GeV} / c^{2}\right)\right)$. The systematic uncertainty is computed by considering the differences between the CTEQ5L and MRST72 PDFs, different $\Lambda_{Q C D}$ values (MRST75 - MRST72) and the sum in quadrature of half the 


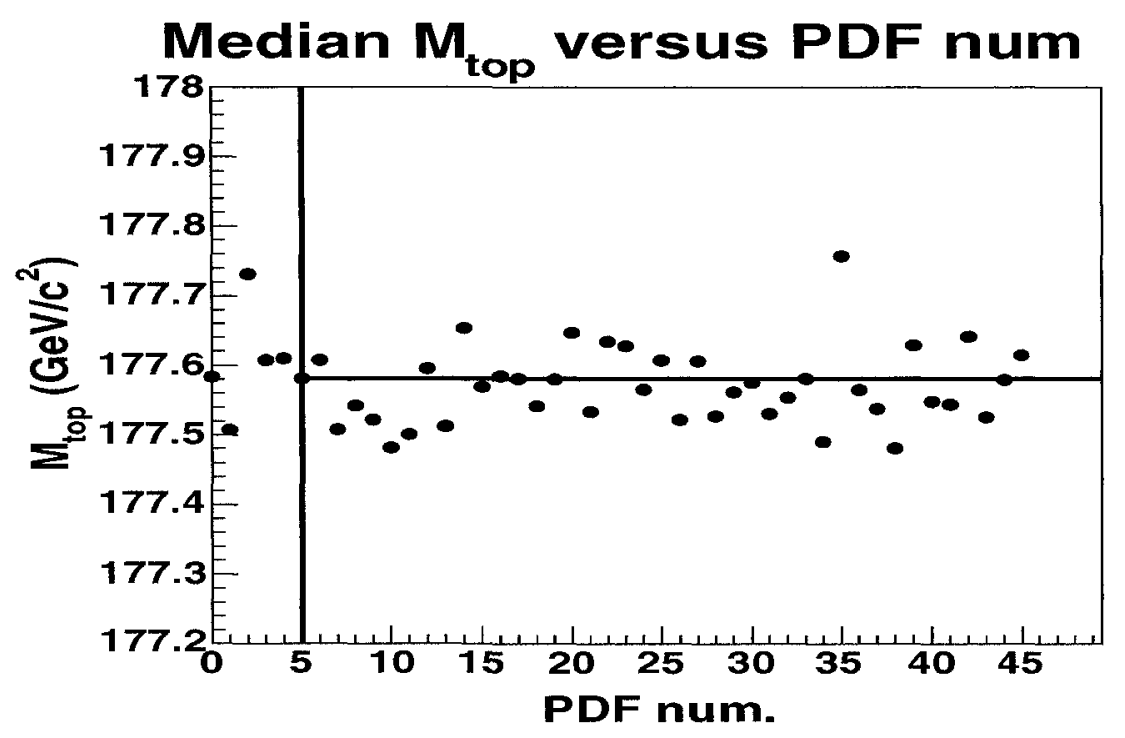

Figure 8.3: Median of fitted $M_{\text {top }}$ from pseudo-experiments versus PDF number (defined in the text). The lines indicate the result for the default CTEQ6M PDFs. All points to the right of the vertical line correspond to $\pm 1 \sigma$ variations of the CTEQ6M PDFs.

difference between the $+1 \sigma$ and $-1 \sigma$ shift of the 20 CTEQ6M uncertainties. The results are summarized in Table 8.3. We add these three contributions in quadrature to obtain a total PDFs uncertainty of $0.3 \mathrm{GeV} / c^{2}$ on $M_{\text {top }}$ and $0.04 \sigma_{c}$ and $0.11 \sigma_{c}$ on JES in the $M_{\text {top }}$ measurement and JES cross-check, respectively.

\begin{tabular}{cccc}
\hline \hline Systematic effect & \multicolumn{2}{c}{$M_{\text {top }}$ Measurement } & JES Cross-Check \\
& $\Delta M_{\text {top }}\left(\mathrm{GeV} / c^{2}\right)$ & $\Delta J E S\left(\sigma_{c}\right)$ & $\Delta J E S\left(\sigma_{c}\right)$ \\
\hline MRST72-CTEQ5L & $-0.08 \pm 0.03$ & $0.002 \pm 0.005$ & $0.003 \pm 0.008$ \\
MRST75-MRST72 & $+0.22 \pm 0.03$ & $0.017 \pm 0.005$ & $0.065 \pm 0.008$ \\
20 uncertainties from CTEQ6M & $+0.20 \pm 0.09$ & $0.032 \pm 0.016$ & $0.086 \pm 0.048$ \\
\hline \hline
\end{tabular}

Table 8.3: Systematic shift in $M_{\text {top }}$ and JES due to different PDFs. 


\begin{tabular}{cccc}
\hline \hline Systematic effect & \multicolumn{2}{c}{$M_{\text {top }}$ Measurement } & JES Cross-Check \\
& $\Delta M_{\text {top }}\left(\mathrm{GeV} / c^{2}\right)$ & $\Delta J E S\left(\sigma_{c}\right)$ & $\Delta J E S\left(\sigma_{c}\right)$ \\
\hline Only $W+$ jets mistags - default & $-0.14 \pm 0.14$ & $-0.031 \pm 0.020$ & $-0.003 \pm 0.035$ \\
Only $W b \bar{b}$ - default & $-0.60 \pm 0.14$ & $-0.006 \pm 0.020$ & $+0.007 \pm 0.035$ \\
$Q^{2}$ scale: $4 M_{W}^{2}-<p_{T}(\text { parton })^{2}>$ & $+0.77 \pm 0.14$ & $+0.098 \pm 0.020$ & $+0.349 \pm 0.034$ \\
Fake electron data - default & $-0.10 \pm 0.14$ & $+0.059 \pm 0.020$ & $+0.151 \pm 0.034$ \\
\hline
\end{tabular}

Table 8.4: Systematic shifts due to variations of the background shapes.

\subsection{Background Shape}

The likelihood fits employ a single shape for the background templates. We evaluate the $M_{\text {top }}$ uncertainties due to the uncertainties in the background shape arising from potential differences in the normalization of the combined tagged background templates, the $Q^{2}$ scale used for the $W$ +jets event generation and the shape of the QCD background.

The combined background templates of the 2-tag, 1-tag(T), and 1-tag(L) subsamples are made out of a combination of a $W b \bar{b}, W+$ jets and single-top MC samples in the expected proportions calculated a priori (see Sec. 4.4). We calculate the uncertainty arising from the normalization of the various background contributions by replacing the combined background template with the templates of the two dominant background sources $(W+$ jets mistag and $W b \bar{b})$. This is done simultaneously for the $m_{t}^{r e c o}$ and $m_{j j}$ templates and for all tagged subsamples. The resulting shifts in $M_{t o p}$ and JES are summarized in Table 8.4. We consider half of the largest shift (or the uncertainty on the shift if the shift itself is insignificant) as the systematic uncertainty: $0.3 \mathrm{GeV} / c^{2}$ on $M_{\text {top }}$ and $0.02 \sigma_{c}$ and $0.02 \sigma_{c}$ on the JES in the $M_{\text {top }}$ measurement and JES cross-check, respectively.

We also consider uncertainties due to the choice of the $Q^{2}$ scale used in the generation of $W+$ jets and $W b \bar{b}$ events. ALPGEN MC samples are generated with four different $Q^{2}$ scales: $Q^{2}=<p_{T}(\text { parton })^{2}>, Q^{2}=M_{W}^{2} / 4, Q^{2}=4 M_{W}^{2}$ and $Q^{2}=M_{W}^{2}+\sum p_{T}(\text { parton })^{2}$ 
(the default one), where $p_{T}$ (parton) is the $p_{T}$ of the partons that are produced in association with the $W$ boson. The largest observed variation is as expected between the two largest variations of the $Q^{2}$ scale, i.e. $Q^{2}=<p_{T}$ (parton $)^{2}>$ and $Q^{2}=4 M_{W}^{2}$ that result in a shift in fitted $M_{\text {top }}$ of $0.8 \mathrm{GeV} / c^{2}$. We take half of the shift as the systematic uncertainty $\left(0.4 \mathrm{GeV} / c^{2}\right)$. The uncertainties on the fitted JES are $0.05 \sigma_{c}$ and $0.17 \sigma_{c}$ for the $M_{t o p}$ measurement and JES cross-check, respectively.

The uncertainty arising from the assumption that the QCD background is well modeled by $W+$ jets MC events is evaluated by studying data containing "fake electrons." Events in the fake electron sample were collected by a trigger selecting QCD multijets events requiring at least 4 jets with $E_{T}>15 \mathrm{GeV}$ and that the sum of the energy deposited in the calorimeters in the event be more than $125 \mathrm{GeV}$. Requirements are applied to one of the jets in these events such that it emulates the characteristics of an electron: $E_{T}(j e t)>20 \mathrm{GeV},|\eta|<1.0$ and a large fraction of energy deposited in the electromagnetic calorimeter $(\mathrm{EM}$ fraction $>0.8)$. We reject real electrons by requiring at least four tracks inside the jet cone and the EM fraction of the jet associated with the electron energy cluster to be less than 0.95 .

The $m_{t}^{\text {reco }}$ and $m_{j j}$ shape reconstructed from the fake electron data are shown in Figs. 8.4 and 8.5 (data points), respectively, along with the background shape for the $W+$ jets MC background events (full line) that is used in this analysis to model the QCD background. Good agreement is found between the two type of events. ${ }^{2}$ The size of the $M_{\text {top }}$ uncertainty induced by this assumption on the background shape is quantified by generating pseudo-experiments where the background shape is replaced by the fake electron shape for the expected QCD background contributions. The results are summarized in Table 8.4 and correspond to systematic uncertainties of $0.1 \mathrm{GeV} / c^{2}$

\footnotetext{
${ }^{2}$ Some disagreement appears at low mass: the fake electron data is shifted slightly higher than the $W+$ jets MC. This is presumably due to the trigger requirement in the multijets sample that the sum of the $E_{T}$ deposited in the event be greater than $125 \mathrm{GeV}$. This cut is not part of the lepton+jets events selection.
} 

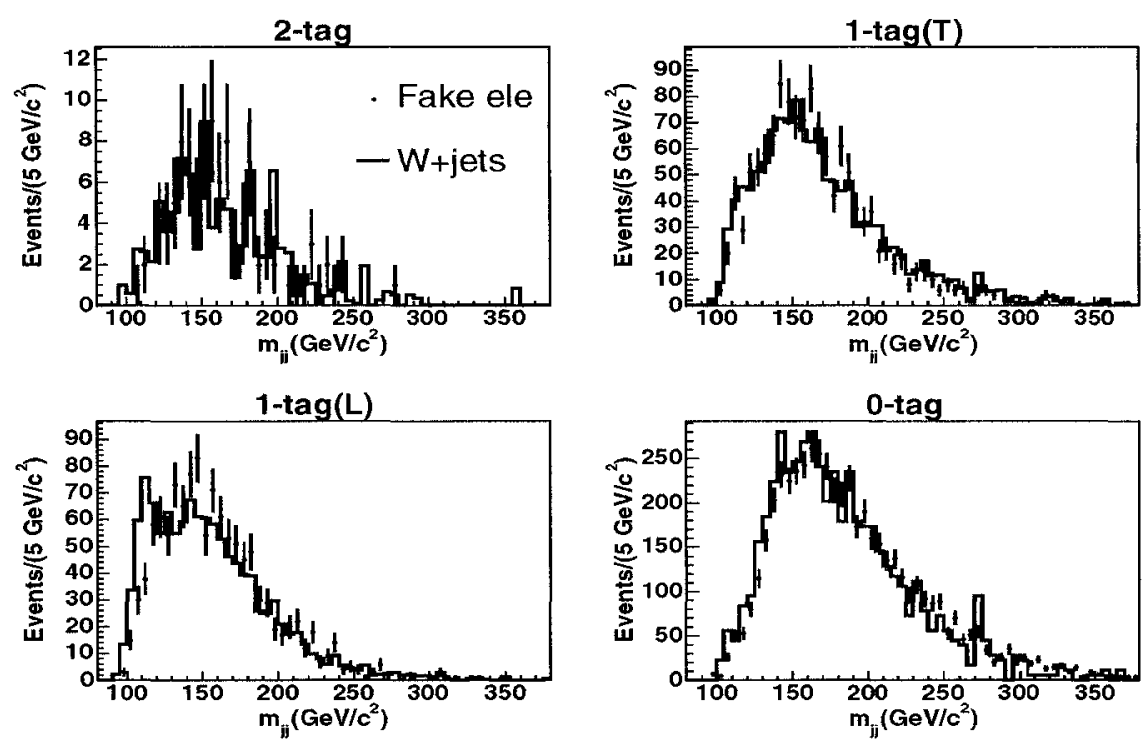

Figure 8.4: Background $m_{t}^{\text {reco }}$ shape for the fake electron data (data points) and the $W+$ jets MC (full line) in 2-tag events (upper-left), 1-tag(T) events (upper-right), 1$\operatorname{tag}(\mathrm{L})$ events (bottom-left) and 0-tag events (bottom-right).

for $M_{t o p}$ and $0.06 \sigma_{c}$ and $0.15 \sigma_{c}$ for the JES from the $M_{t o p}$ measurement and the JES cross-check, respectively.

We add these contributions in quadrature to obtain a total background shape uncertainty of $0.5 \mathrm{GeV} / c^{2}$ on $M_{\text {top }}$ and $0.08 \sigma_{c}$ and $0.23 \sigma_{c}$ on the JES in the $M_{\text {top }}$ measurement and JES cross-check, respectively.

\subsection{Background Jet Energy Scale}

As explained in Sec. 5.6, the background templates are created independent of JES. We consider the systematic uncertainty due to JES variations in the background separately. We treat it as correlated to the JES uncertainty returned by the likelihood fits. This is estimated from pseudo-experiments where JES for both the signal and background shapes are shifted by $\pm 1 \sigma_{c}$, and look at the differences in fitted $M_{\text {top }}$ and JES with respect to 

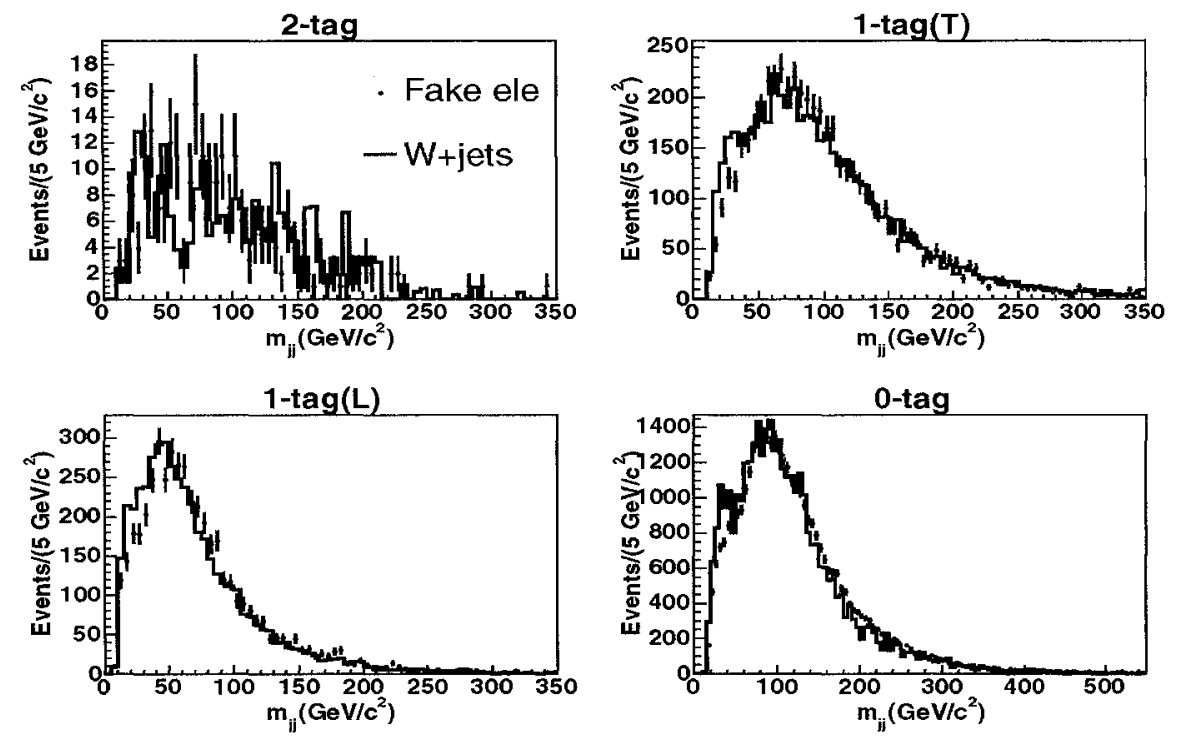

Figure 8.5: Background $m_{j j}$ shape for the fake electron data (data points) and the $W+$ jets MC (full line) in 2-tag events (upper-left), 1-tag(T) events (upper-right), 1-tag(L) events (bottom-left) and 0-tag events (bottom-right).

the default case where only the signal shape is shifted. The background shapes employed are the ones coming from the $W+$ jets background where the available MC statistics is the most abundant. The results are given in Table 8.5. Very small differences with the default procedure are observed. We take half the difference between the results of the $\pm 1 \sigma_{c}$ pseudo-experiments as the systematic uncertainty, i.e. $0.04 \mathrm{GeV} / c^{2}$ on $M_{\text {top }}$ and $0.033 \sigma_{c}$ and $0.074 \sigma_{c}$ on the JES in the $M_{\text {top }}$ measurement and JES cross-check, respectively. Since this uncertainty is correlated to JES, we add it linearly to the JES component of the total uncertainty obtained in the data as described in Sec. 9.1.1 and 9.2.1 for the JES cross-check and $M_{t o p}$ measurement, respectively. 


\begin{tabular}{cccc}
\hline \hline Systematic effect & \multicolumn{2}{c}{$M_{\text {top }}$ Measurement } & JES Cross-Check \\
& $\Delta M_{t o p}\left(\mathrm{GeV} / c^{2}\right)$ & $\Delta J E S\left(\sigma_{c}\right)$ & $\Delta J E S\left(\sigma_{c}\right)$ \\
\hline$+1 \sigma_{c}$ background - default & $-0.07 \pm 0.06$ & $-0.021 \pm 0.011$ & $-0.052 \pm 0.016$ \\
$-1 \sigma_{c}$ background - default & $+0.02 \pm 0.06$ & $+0.044 \pm 0.011$ & $+0.096 \pm 0.015$ \\
\hline \hline
\end{tabular}

Table 8.5: Systematic shifts due to variations of the JES for the background shapes.

\subsection{Monte Carlo Statistics}

The top quark mass is measured using signal and background mass shapes extracted from MC samples with limited statistics (typically a few hundred thousand events). We estimate the uncertainty due to the finite MC statistics separately for signal and background events. For signal we consider the reconstructed mass histogram for one mass point $\left(M_{t o p}=180 \mathrm{GeV} / c^{2}\right)$ to which we fluctuate the number of entries in each bin using Poisson statistics to create a new (statistically fluctuated) template. The operation is applied to the $m_{t}^{\text {reco }}$ and $m_{j j}$ templates of each subsample simultaneously and repeated a hundred times. A set of ten thousand pseudo-experiments is generated randomly from each of the one hundred statistically fluctuated templates. The resulting distribution of fitted $M_{t o p}$ is shown in Fig. 8.6. This distribution is fitted to a Gaussian function to obtain a width of $0.34 \pm 0.02 \mathrm{GeV} / \mathrm{c}^{2}$. The uncertainty due to the limited pseudo-experiments statistics $\left(0.10 \mathrm{GeV} / c^{2}\right)$ is subtracted in quadrature to obtain $0.32 \mathrm{GeV} / c^{2}$, which we take as the systematic uncertainty due to limited signal Monte Carlo statistics.

The same procedure is applied to the $W b \bar{b}$ background shape for tagged events and $W+$ jets background for the 0 -tag sample to get $0.27 \pm 0.02 \mathrm{GeV} / c^{2}$. This is an overestimate of the uncertainty since it is evaluated using only one background source per subsample. In reality additional statistics is available for other background samples (for instance the $W+$ jets mistag). We thus take half of this value to get the limited MC uncertainty of the background samples: $0.13 \mathrm{GeV} / c^{2}$. We add in quadrature the signal and background MC statistics uncertainty to get a total uncertainty of $0.3 \mathrm{GeV} / c^{2}$. A 


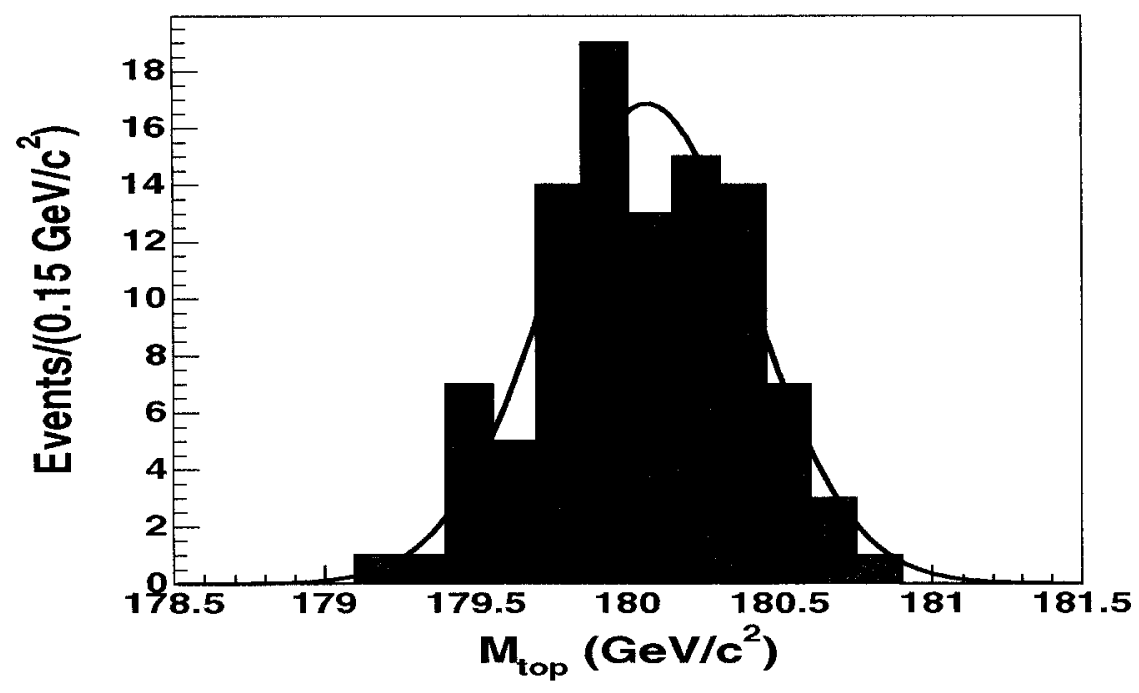

Figure 8.6: Median of fitted $M_{t o p}$ distribution of 10,000 pseudo-experiments for 100 statistically fluctuated signal templates (with $M_{t o p}=180 \mathrm{GeV} / c^{2}$ ).

similar procedure is performed to get JES uncertainties of $0.05 \sigma_{c}$ and $0.09 \sigma_{c}$ for the $M_{\text {top }}$ measurement and JES cross-check, respectively.

\subsection{B-tagging}

Discrepancies between the SECVTX $b$-tagging efficiency in data and MC can bias the measurement. In particular, biases in the $p_{T}$-dependence of the efficiency directly affects the shape of the mass templates and thus the $M_{\text {top }}$ measurement.

The ratio of the data and MC b-tagging efficiencies (scale factor) is consistent with a constant as a function of jet $p_{T}[21]$. The positive and negative statistical variations of the slope of the scale factor as a function of jet $p_{T}, \epsilon^{+}$and $\epsilon^{-}$, are extracted and correspond to

$$
\begin{aligned}
& \epsilon^{+}=0.842+0.00118 p_{T}, \\
& \epsilon^{-}=0.977-0.00118 p_{T} .
\end{aligned}
$$


New signal and background mass shapes are reconstructed with these variations, and the pseudo-experiments are then generated. We take half of the difference of the fitted $M_{\text {top }}$ and JES between the positive and negative variations of the scale factor slope as the systematic uncertainties: $0.1 \mathrm{GeV} / c^{2}$ for $M_{\text {top }}$ and $0.01 \sigma_{c}$ for the JES from both the $M_{\text {top }}$ measurement and JES cross-check.

\subsection{0 $M_{\text {top }}$ Uncertainty for the JES Cross-Check}

The top quark mass is fixed to $178 \mathrm{GeV} / c^{2}$ when performing the JES cross-check. Since the $m_{j j}$ templates display a modest dependence on $M_{t o p}$ (see e.g. Figs. 6.3 and 6.5), we need to consider an uncertainty for the JES cross-check due to the experimental uncertainty on $M_{t o p}$. This is estimated by varying the top quark mass by $5 \mathrm{GeV} / c^{2}$, corresponding approximately to the uncertainty to the current world average [6]. We observe a shift of $0.36 \sigma_{c}$ on the fitted JES, which we take as the systematic uncertainty.

\subsection{Summary}

The summary of the uncorrelated systematic uncertainties on the fitted $M_{\text {top }}$ and JES are given in Table 8.6. The total systematic uncertainty, apart from the JES uncertainty, is $1.3 \mathrm{GeV} / c^{2}$ for $M_{\text {top }}$ and $0.33 \sigma_{c}$ and $0.68 \sigma_{c}$ for JES obtained in the $M_{\text {top }}$ measurement and JES cross-check, respectively. The background JES uncertainty is considered as correlated to the fitted JES uncertainty and is treated as such after fitting the data as described in Sec. 9.2.1 and 9.1.1 for the JES cross-check and the $M_{t o p}$ measurement, respectively. 


\begin{tabular}{lccc}
\hline \hline Systematic effect & $M_{\text {top }}$ Measurement & JES Cross-Check \\
& $\Delta M_{\text {top }}\left(\mathrm{GeV} / c^{2}\right)$ & $\Delta J E S\left(\sigma_{c}\right)$ & $\Delta J E S\left(\sigma_{c}\right)$ \\
\hline$b$-jets modeling & 0.6 & 0.25 & - \\
Method & 0.5 & 0.02 & 0.04 \\
ISR & 0.4 & 0.08 & 0.24 \\
FSR & 0.6 & 0.06 & 0.24 \\
Generator & 0.2 & 0.15 & 0.38 \\
PDFs & 0.3 & 0.04 & 0.11 \\
Background shape & 0.5 & 0.08 & 0.23 \\
MC statistics & 0.3 & 0.05 & 0.09 \\
$B$-tagging & 0.1 & 0.01 & 0.01 \\
$M_{\text {top }}$ (JES cross-check only) & - & - & 0.36 \\
\hline Total & 1.3 & 0.33 & 0.68 \\
\hline \hline
\end{tabular}

Table 8.6: Summary of the uncorrelated systematic uncertainties. The JES uncertainty on $M_{t o p}$ is extracted from the likelihood fit and not included in this summary. 


\section{Chapter 9}

\section{The Top Quark Mass Measurement}

The full machinery for measuring the top quark mass is now in place. Templates of the reconstructed top quark and $W$ boson mass are created from SM $t \bar{t}$ events as a function of the true top quark mass and JES. The likelihood fits have been shown to give unbiased central values and uncertainties of the parameters of interest for large ranges of $M_{t o p}$ and JES. We expect a statistical+JES uncertainty on $M_{\text {top }}$ of roughly $4.3 \mathrm{GeV} / c^{2}$ with the current dataset. The systematic uncertainties are estimated and are small compared to the statistical and JES uncertainties coming from the likelihood fits. All the tools are ready to be applied to the real data. We first check that the a priori jet energy calibration and $W \rightarrow j j$ measurement of JES are in agreement with each other by performing the JES cross-check before combining them to perform the full $M_{\text {top }}$ measurement.

The dataset used for the measurement consists of data collected by the CDF detector between March 2002 and August 2004 corresponding to an integrated luminosity of $\int \mathcal{L} d t=318 \mathrm{pb}^{-1}$. The data sample has been validated thoroughly and shown to be suitable for measuring the top quark mass [59]. A total of 138 and 165 events enter the $m_{t}^{r e c o}$ and $m_{j j}$ samples, respectively. The distribution of the events in each of the four subsamples is given in Table 4.1. 


\subsection{Application of the JES Cross-Check to Data}

The $m_{j j}$ distributions reconstructed in the data are shown in Fig. 9.1. The application of the JES cross-check likelihood fit yields JES $=-0.76_{-0.97}^{+0.97} \sigma_{c}$ where the uncertainties are not corrected for $68 \%$ confidence level coverage. The shape of the MC templates corresponding to the best fit are overlaid on top of the histograms of Fig. 9.1. A good agreement between the data and MC shape is observed. Kolmogorov-Smirnov tests have been performed to compare the data and $\mathrm{MC} m_{j j}$ distributions and yield probabilities of $57 \%, 55 \%, 84 \%$ and $79 \%$ for the 2-tag, 1-tag(T), 1-tag(L) and 0-tag subsamples, respectively.

The shape of the negative log-likelihood function $(-\ln \mathcal{L})$ as a function of JES is shown in Fig. 9.2 (where the likelihood is re-optimized at each point of the curve). The shape is approximately parabolic over the full range considered. We apply the uncertainty scale factor of 1.027 extracted in Sec. 7.2 .3 to obtain:

$$
\mathrm{JES}=-0.76 \pm 1.00 \text { (stat.) } \sigma_{c}
$$

The distribution of the expected negative and positive uncertainties based on the number of observed events and expected number of background events are shown in Fig. 9.3. The uncertainty obtained in the data (indicated by the arrows) are consistent with the Monte Carlo expectations: $46 \%$ of pseudo-experiments have a smaller average uncertainty.

The number of signal and background events returned by the likelihood fit are given in Table 9.1. The fitted number of background events are in good agreement with the $a$ priori calculation also given in Table 9.1 as expected since they are constrained to these values in the likelihood fit. In the 0-tag subsample, the number of background events is left unconstrained and the likelihood fit chooses a normalization that corresponds to a signal over background ratio of $2.5_{-1.6}^{+1.7}$.

The stability of the background normalizations is checked by performing a different fit where the background is left unconstrained for all subsamples (i.e. the term $\mathcal{L}_{b g}$ in the 

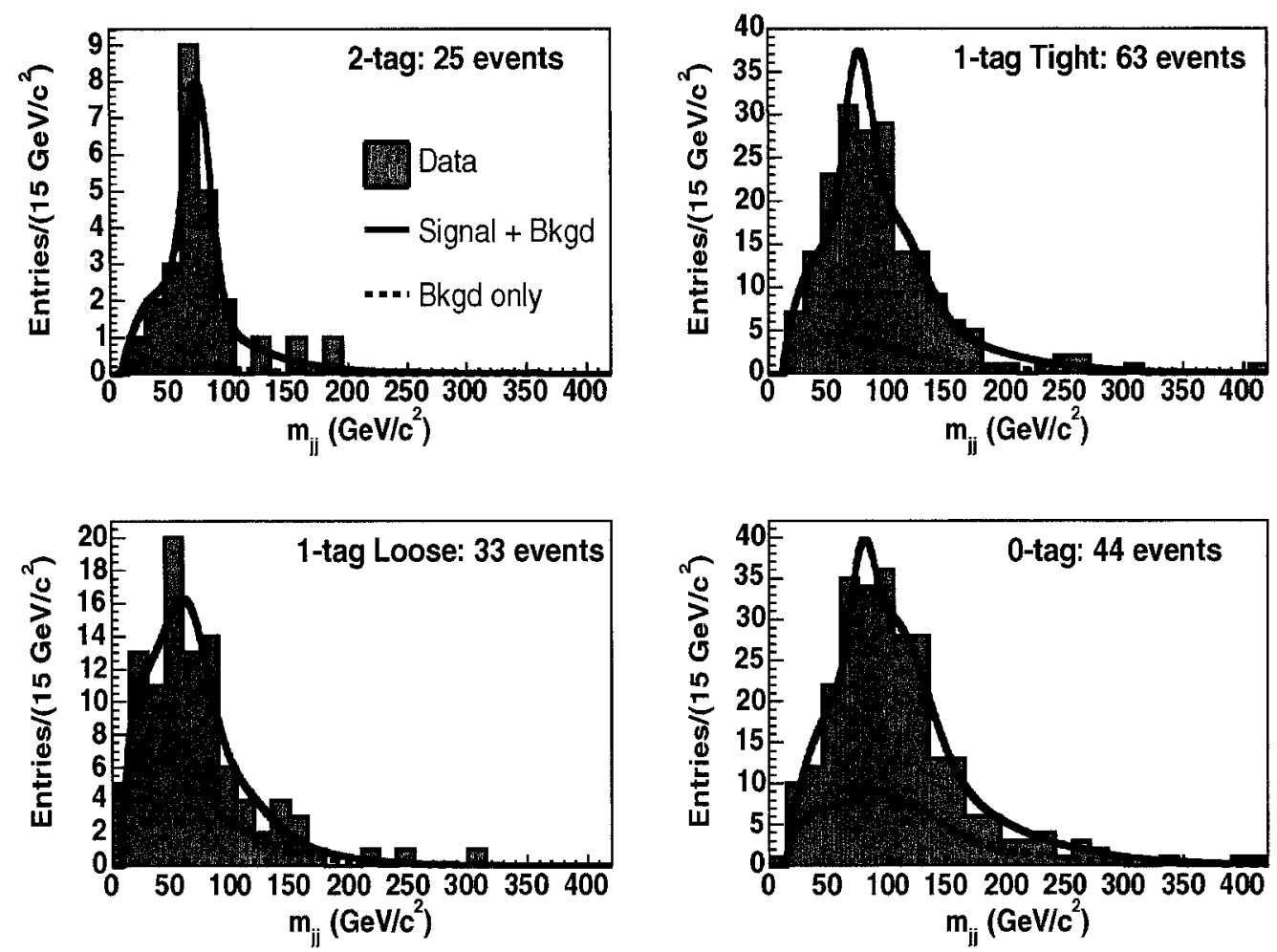

Figure 9.1: Data $m_{j j}$ distributions for the 2-tag (upper-left), 1-tag(T) (upper-right), 1-tag(L) (lower-left) and 0-tag (lower-right) subsamples. The signal and background template shapes corresponding to the best fit of the JES cross-check are overlaid on the histograms. The value of $M_{\text {top }}$ has been constrained to $178 \mathrm{GeV} / c^{2}$.

subsample likelihood of Eqn. 7.1 is ignored). The fitted number of signal and background events in this case is solely determined by the $m_{t}^{r e c o}$ and $m_{j j}$ shape and constitute a good cross-check of the a priori background calculation for the tagged samples. The fitted JES is $-0.98_{-1.03}^{+1.02} \sigma_{c}$, in good agreement with the default fit. The fitted number of events for the background unconstrained fit are given in Table 9.2. The uncertainties are relatively large but the fitted number of signal and background events are in good agreement with the a prioricalculation, giving us more confidence that the normalization of the background for the JES cross-check is reasonable.

We check that the fitted JES from the different subsamples are consistent with each 


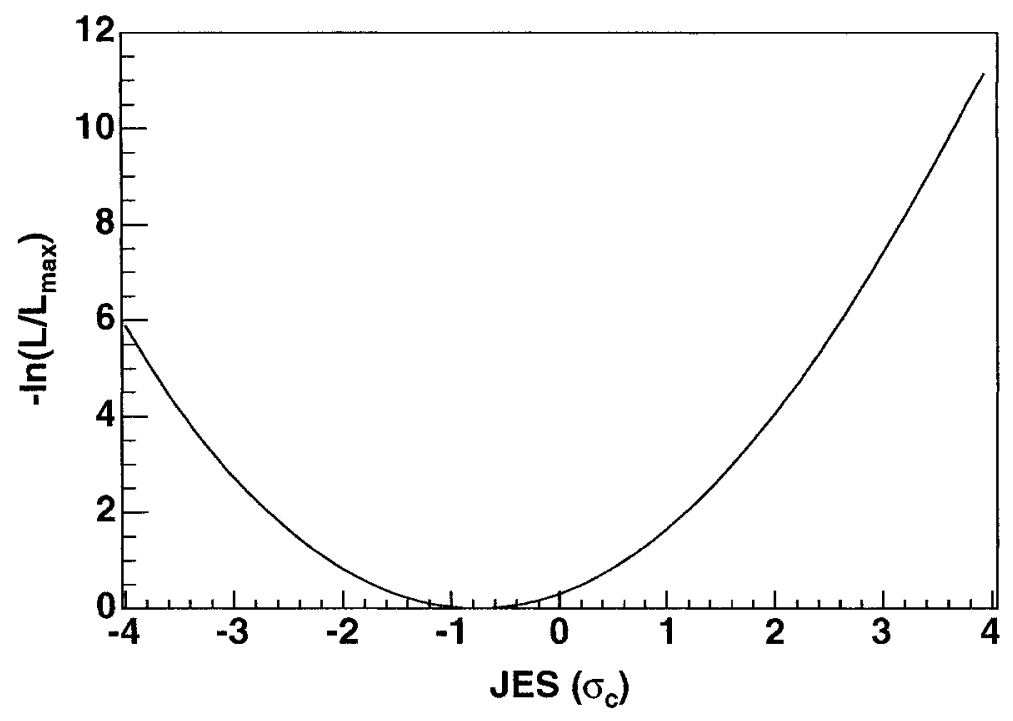

Figure 9.2: Negative log-likelihood function as a function of JES for the JES cross-check.

others by minimizing separately the subsample's likelihood functions. The results are given in Table 9.3 where the uncertainties are not corrected for $68 \%$ confidence level coverage. The $-\ln \mathcal{L}$ of each subsample as a function of JES are shown in Fig. 9.4 for the range from $-4 \sigma_{c}$ to $+4 \sigma_{c}$. The 2 -tag and 1-tag(T) yield the most precise measurements both with uncertainties of about $1.5 \sigma_{c}$. A second minimum appears for the 0-tag subsample near $-5 \sigma_{c}$ (not shown in Fig. 9.4) that is only about 1 unit larger than the central value of the combined fit, which corresponds to a discrepancy of less than $1.5 \sigma$. We thus conclude that there is no significant discrepancy for the 0-tag sample.

\subsubsection{Final Result for the JES Cross-Check}

We add the systematic uncertainties found in Chapter 8 to get the final result for the JES cross-check. The background JES uncertainty $\left(0.074 \sigma_{c}\right)$ is added linearly to the statistical uncertainty and the uncorrelated systematic uncertainties $\left(0.68 \sigma_{c}\right)$ are added in quadrature. The final result is:

$$
\mathrm{JES}=-0.76 \pm 1.27 \sigma_{c},
$$




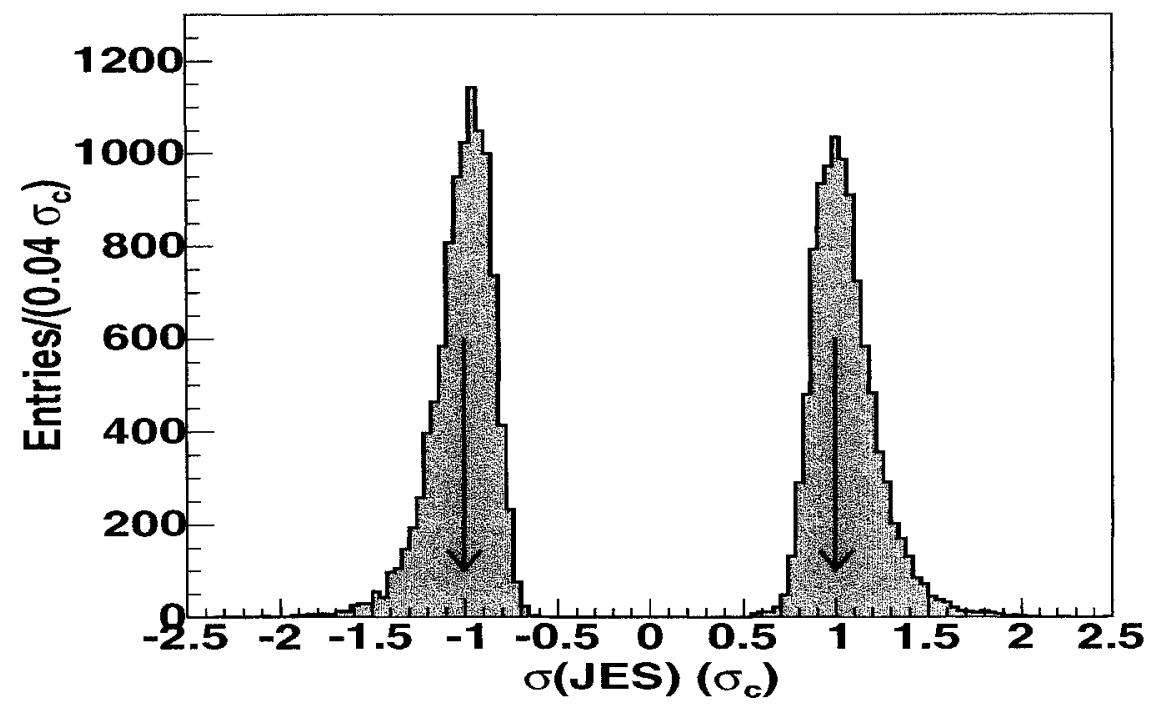

Figure 9.3: Negative and positive uncertainties based on the number of observed events for the JES cross-check. The arrows indicate the uncertainties obtained in data.

where $M_{\text {top }}$ was fixed to $M_{\text {top }}=178 \mathrm{GeV} / c^{2}$ in the fit. We conclude that the JES as determined by $W \rightarrow j j$ decays is in good agreement with the CDF jet energy calibration (JES $=0 \pm 1 \sigma_{c}$ ). We then combine these two estimates to perform the full $M_{t o p}$ measurement.

\subsection{Application of the $M_{t o p}$ Measurement to Data}

The $m_{t}^{r e c o}$ and $m_{j j}$ distributions reconstructed in the data are shown in Figs. 9.5 and 9.6, respectively. The signal and background templates for the best fit are overlaid on top of the histograms. Kolmogorov-Smirnov tests have been performed to compare the data and MC $m_{t}^{r e c o}$ distributions and yield probabilities of $60 \%, 75 \%, 59 \%$ and $37 \%$ for the 2-tag, 1-tag(T), 1-tag(L) and 0-tag subsamples, respectively (the probabilities for the $m_{j j}$ distributions are very similar to the ones given for the JES cross-check in Sec. 9.1). 


\begin{tabular}{ccccc}
\hline \hline Subsample & Observed & Fitted $n_{s}$ & Fitted $n_{b}$ & $n_{b}$ (constr) \\
\hline $2-\operatorname{tag}$ & 25 & $23.3_{-5.0}^{+5.0}$ & $1.9_{-0.5}^{+0.5}$ & $1.89 \pm 0.52$ \\
$1-\operatorname{tag}(\mathrm{T})$ & 63 & $51.8_{-8.0}^{+8.0}$ & $10.6_{-1.7}^{+1.7}$ & $10.4 \pm 1.7$ \\
$1-\operatorname{tag}(\mathrm{L})$ & 33 & $15.0_{-5.6}^{+5.6}$ & $15.3_{-2.3}^{+2.3}$ & $14.3 \pm 2.5$ \\
$0-\operatorname{tag}$ & 44 & $30.4_{-9.1}^{+9.1}$ & $13.6_{-7.6}^{+8.7}$ & None \\
\hline \hline
\end{tabular}

Table 9.1: The fitted number of signal $\left(n_{s}\right)$ and background events $\left(n_{b}\right)$ in each subsample for the JES cross-check. Also given are the expected number of background event that $n_{b}$ is constrained to in the likelihood fit $\left(n_{b}(\right.$ constr $\left.)\right)$.

\begin{tabular}{ccc}
\hline \hline Subsample & Fitted $n_{s}$ & Fitted $n_{b}$ \\
\hline 2-tag & $25.0_{-18.9}^{+18.9}$ & $0.0_{-0.0}^{+5.4}$ \\
$1-\operatorname{tag}(\mathrm{T})$ & $46.6_{-11.3}^{+11.3}$ & $16.4_{-9.8}^{+10.9}$ \\
$1-\operatorname{tag}(\mathrm{L})$ & $7.5_{-7.4}^{+7.4}$ & $25.5_{-8.3}^{+8.3}$ \\
$0-\operatorname{tag}$ & $30.4_{-8.7}^{+8.7}$ & $13.6_{-7.6}^{+8.6}$ \\
\hline \hline
\end{tabular}

Table 9.2: The fitted number of signal $\left(n_{s}\right)$ and background events $\left(n_{b}\right)$ in each subsample for the JES cross-check where the background is left unconstrained for all subsamples.

The minimization of the $M_{\text {top }}$ measurement likelihood yields

$$
\begin{gathered}
\left.M_{\text {top }}=173.5_{-3.6}^{+3.7} \text { (stat. }+\mathrm{JES}\right) \mathrm{GeV} / c^{2}, \\
\mathrm{JES}=-0.10_{-0.80}^{+0.78} \sigma_{c},
\end{gathered}
$$

after scaling by the uncertainty scale factors of 1.027 and 1.013 respectively for $M_{t o p}$ and JES extracted in Sec. 7.3.3. The shape of the negative log-likelihood as a function of $M_{\text {top }}$ and JES is shown in Fig. 9.7 (where the likelihood is re-optimized at each point of the curves). The anti-correlation between the fitted $M_{t o p}$ and JES obtained from the $m_{t}^{r e c o}$ and $m_{j j}$ distributions can be clearly observed.

The measured $M_{t o p}$ is approximately $0.8 \sigma$ lower but consistent with the Run I world average $M_{t o p}=178.0 \pm 4.3 \mathrm{GeV} / c^{2}$. The central value of the fitted JES is higher than for 


\begin{tabular}{cc}
\hline \hline Subsample & $\operatorname{JES}\left(\sigma_{c}\right)$ \\
\hline 2-tag & $-1.43_{-1.34}^{+1.50}$ \\
1-tag(T) & $0.53_{-1.58}^{+1.44}$ \\
1-tag(L) & $2.16_{-3.25}^{+3.79}$ \\
$0-\operatorname{tag}$ & $-5.64_{-1.34}^{+0.86}$ \\
\hline
\end{tabular}

Table 9.3: Results of the JES cross-check in each subsample. The uncertainties are not corrected for $68 \%$ confidence level coverage.

the JES cross-check since the fitted $M_{\text {top }}$ is lower than the world average that has been used for the JES cross-check. However, both measurements show good agreement with the a priori JES calibration (JES $=0 \pm 1 \sigma_{c}$ ).

The distributions of the expected negative and positive uncertainties based on the number of observed events and expected number of background events are shown in Fig. 9.8 for the fitted $M_{\text {top }}$ (left plot) and JES (right plot). The uncertainties obtained in the data are consistent with the Monte Carlo expectations: $9 \%$ and $66 \%$ of pseudoexperiments have smaller average $M_{\text {top }}$ and JES uncertainties, respectively.

The number of signal and background events in the $m_{j j}$ samples resulting from the full $M_{t o p}$ measurement fit are given in Table 9.4. Good agreement is observed with the results obtained for the JES cross-check (see Table 9.1). As in the JES cross-check case, the background normalization is further checked by performing a fit where the background is left unconstrained for each subsample. The minimization yields $M_{t o p}=$ $173.3_{-3.4}^{+3.5}$ (stat. $\left.+\mathrm{JES}\right) \mathrm{GeV} / c^{2}$ and JES $=-0.10_{-0.77}^{+0.77} \sigma_{c}$, where the uncertainties are not corrected for $68 \%$ confidence level coverage. This is in very good agreement with the background constrained fit but with smaller uncertainties since the likelihood fit chooses more signal events in the 2-tag and 1-tag(T) subsamples when the background is left unconstrained. The fitted number of signal and background events for this fit are given in Table 9.5. The observed number of events agree with the ones obtained 

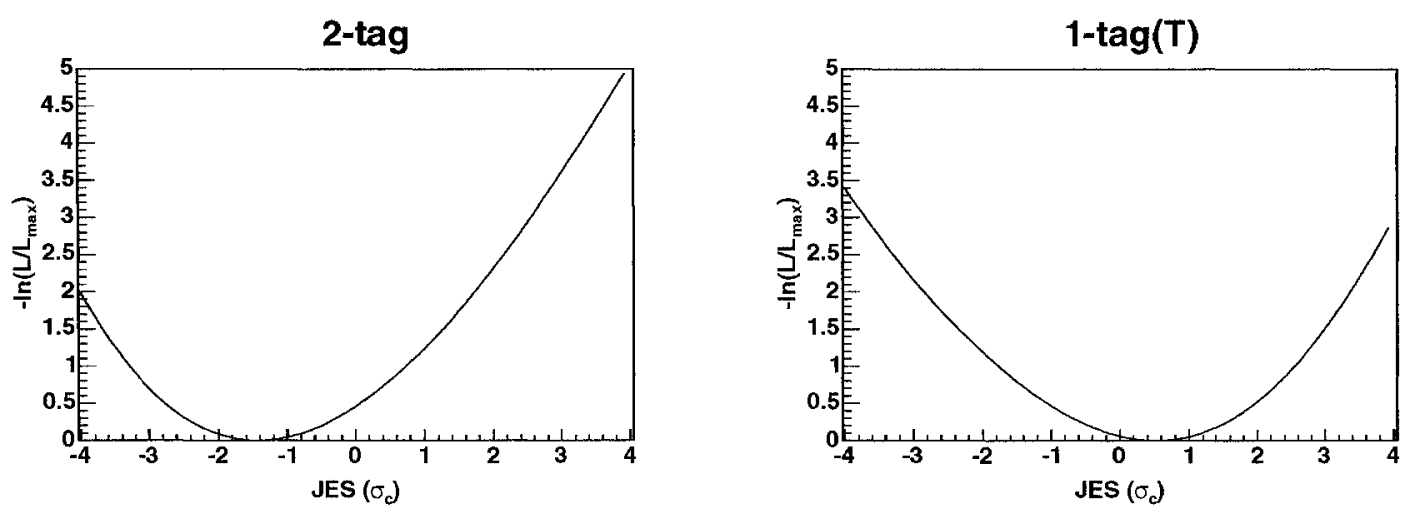

1-tag(L)
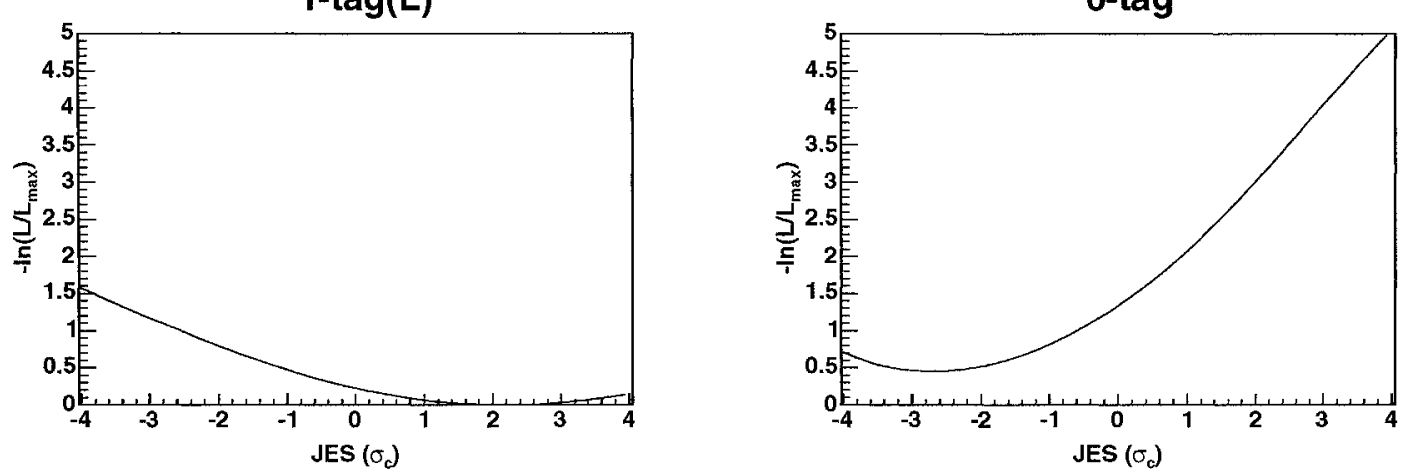

Figure 9.4: Negative log-likelihood as a function of JES for the JES cross-check for the 2-tag (upper-left), 1-tag(T) (upper-right), 1-tag(L) (bottom-left) and 0-tag subsamples.

for the JES cross-check under the same conditions (see Table 9.2), suggesting that the constraints from the $m_{t}^{r e c o}$ and $m_{j j}$ shape (for the $M_{t o p}$ measurement) on the background normalization are in good agreement with the ones obtained using the $m_{j j}$ shape only (for the JES cross-check). The resulting number of events are also in good agreement with the background calculation given in Table 9.4 .

The $M_{t o p}$ measurement is also performed for each subsample separately. The negative log-likelihood as a function of $M_{t o p}$ and JES for each subsample is shown in Fig. 9.9 (where the a priori JES calibration is applied to each subsample individually). The numerical results are given in Table 9.6. Good agreement is observed between all subsamples and with the combined result except for an apparent discrepancy for the fitted $M_{\text {top }}$ in the 0 -tag subsample. As can be seen in Fig. 9.9, the shape of $-\ln \mathcal{L}$ as a function of $M_{\text {top }}$ for 

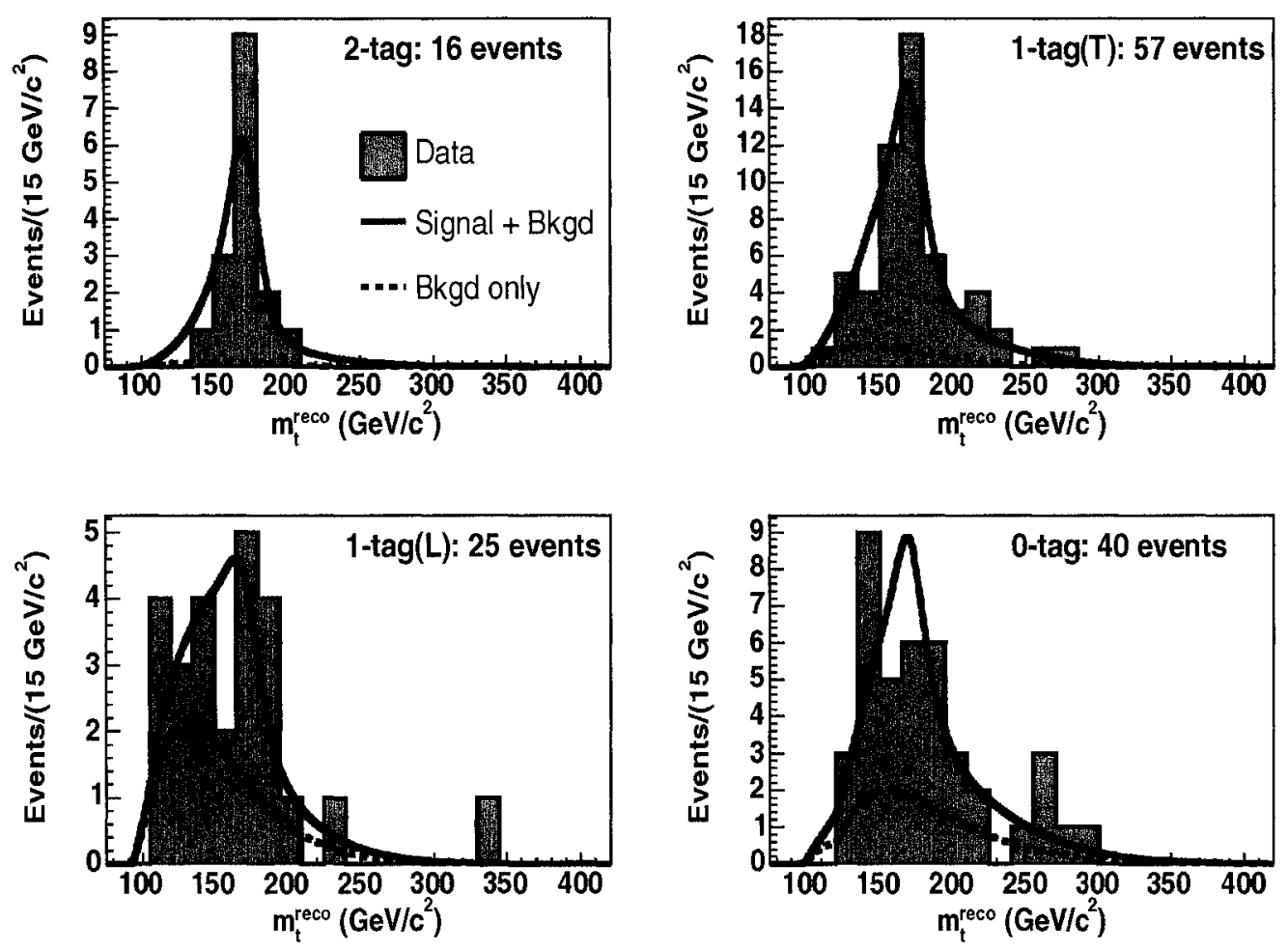

Figure 9.5: Data $m_{t}^{\text {reco }}$ distributions for the 2-tag (upper-left), 1-tag(T) (upper-right), 1$\operatorname{tag}(\mathrm{L})$ (lower-left) and 0-tag (lower-right) subsamples. The template mass distributions for the best fit of the $M_{\text {top }}$ measurement for signal (solid) and background (dashed) are overlaid on the histograms. Both $M_{t o p}$ and JES are free parameters in the likelihood fit.

the 0-tag subsample displays only a mild dependence over the range considered. Indeed, the value of the log-likelihood versus $M_{\text {top }}$ (not shown) near the combined result $\left(M_{\text {top }} \approx\right.$ $173.5 \mathrm{GeV} / c^{2}$ ) differs only by 0.5 unit compared to the most probable value for that subsample $\left(M_{\text {top }} \approx 150 \mathrm{GeV} / c^{2}\right)$ which corresponds to a discrepancy of approximately one standard deviation.

We also perform a fit where the information from the a priori JES calibration is omitted. The minimized likelihood yields:

$$
M_{\text {top }}=174.0 \pm 4.5 \mathrm{GeV} / c^{2}
$$



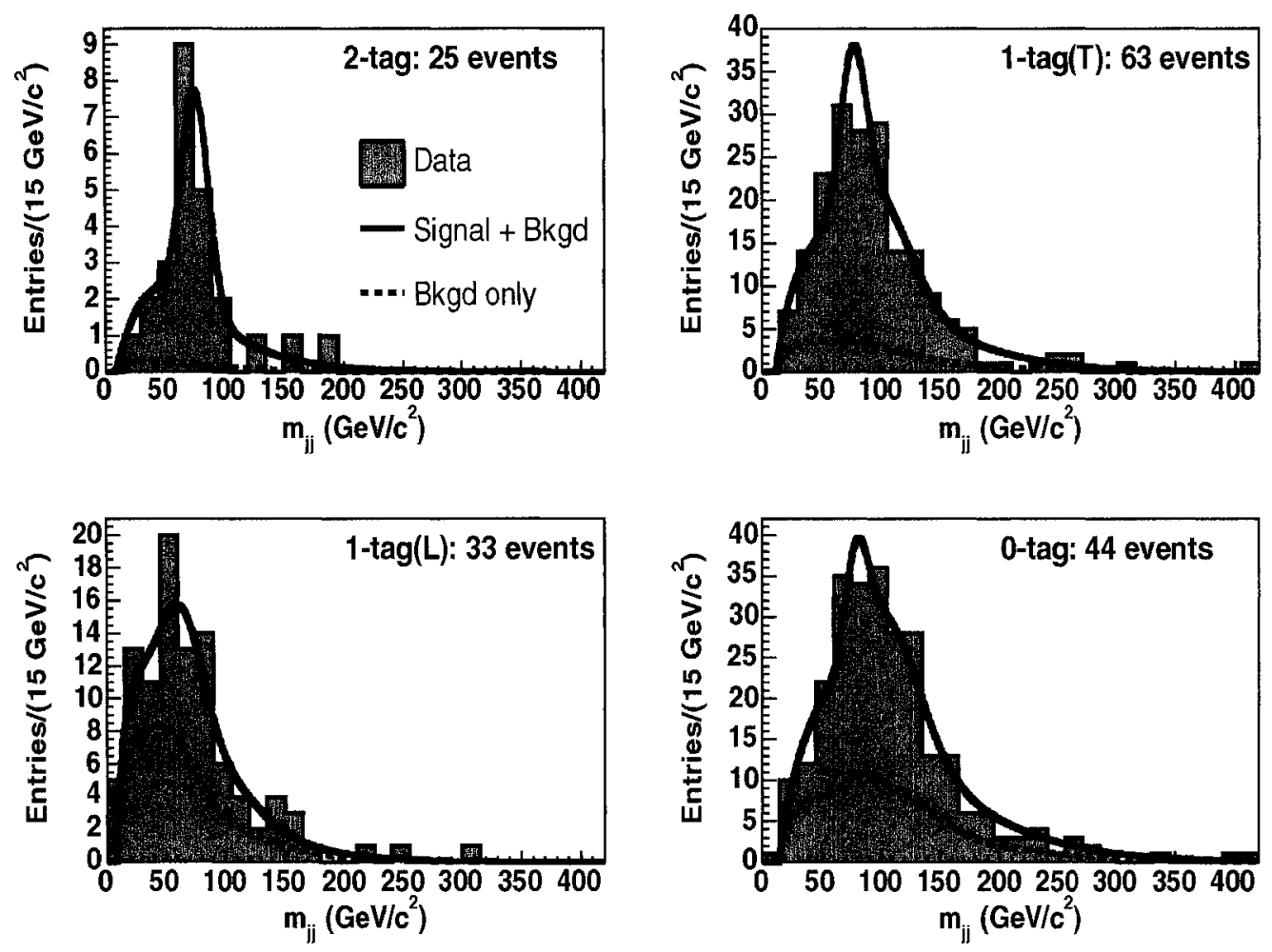

Figure 9.6: Data $m_{j j}$ distributions for the 2-tag (upper-left), 1-tag(T) (upper-right), 1$\operatorname{tag}(\mathrm{L})$ (lower-left) and 0-tag (lower-right) subsamples. The template mass distributions for the best fit of the $M_{\text {top }}$ measurement for signal (solid) and background (dashed) are overlaid on the histograms. Both $M_{t o p}$ and JES are free parameters in the likelihood fit.

$$
\mathrm{JES}=-0.25 \pm 1.22 \sigma_{c}
$$

where the uncertainties are not scaled for $68 \%$ confidence level coverage. The results are in good agreement with the ones obtained for the default case but with larger uncertainties as expected.

In addition, we perform a fit where the JES is fixed and only the $m_{t}^{\text {reco }}$ templates are used to measure $M_{\text {top }}$. This corresponds to the traditional top quark mass template analysis [51]. The minimized likelihood yields:

$$
M_{\text {top }}=173.3_{-2.7}^{+2.8} \text { (stat.) } \mathrm{GeV} / c^{2}
$$




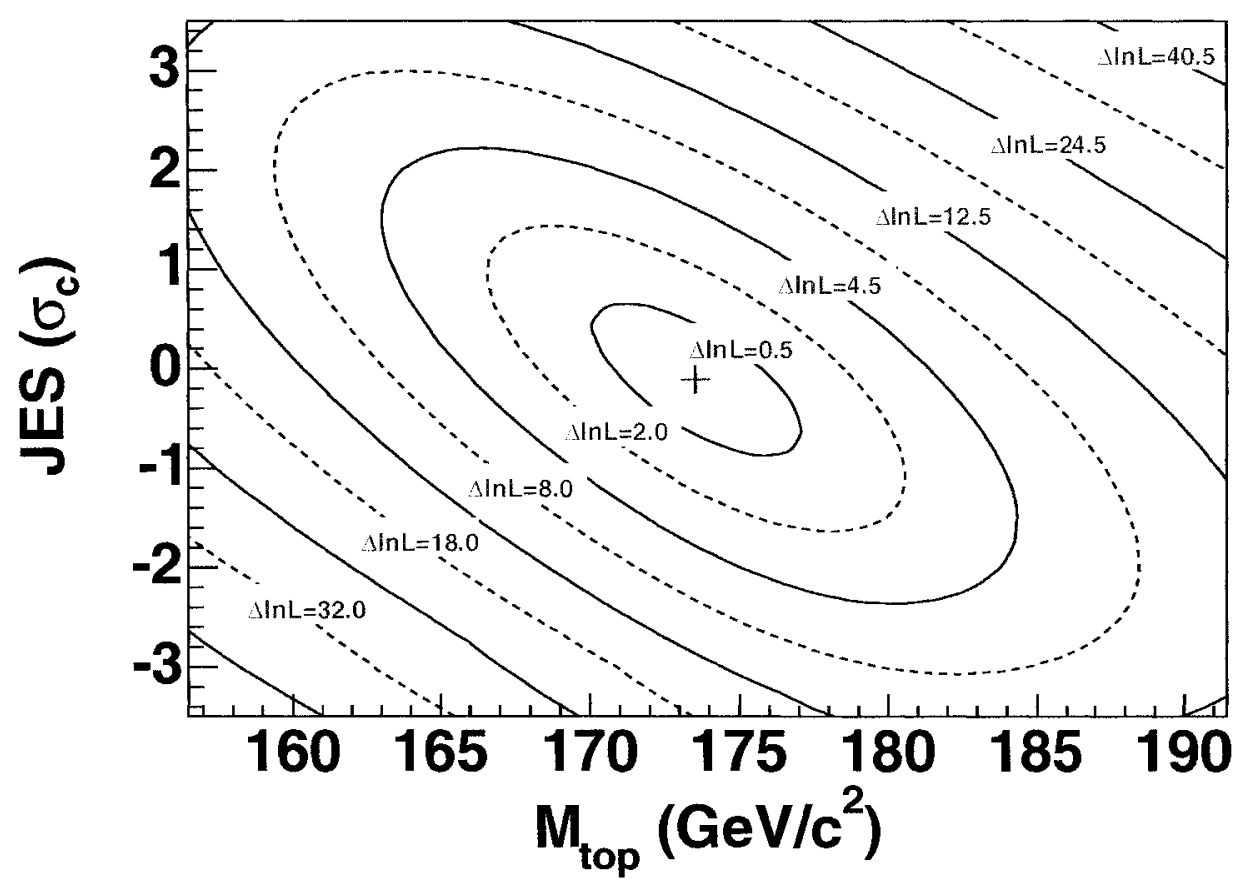

Figure 9.7: Negative log-likelihood as a function of $M_{t o p}$ and JES for the $M_{t o p}$ measurement. The increments in log-likelihood are chosen to represent the one-dimensional standard deviation of the parameters. (To avoid confusion, we don't label the twodimensional curves $1 \sigma, 2 \sigma$, etc, since the equivalent coverage in the multi-dimensional space of parameters requires larger variations of the log-likelihood (see e.g. Table 31.2 of $[5]))$.

that is again in good agreement with the default result.

Finally, it is interesting to untangle the statistical and JES components of the total fitted uncertainty. We do so by fixing JES to the value obtained in the default fit (JES $\left.=-0.10 \sigma_{c}\right)$ and re-perform the $M_{t o p}$ measurement to obtain:

$$
M_{\text {top }}=173.5_{-2.6}^{+2.7} \text { (stat.) } \mathrm{GeV} / c^{2}
$$

where we assume the same uncertainty scale factor of 1.027 can be applied. As expected, the likelihood fit chooses the same central value of $M_{t o p}$. We subtract in quadrature the 

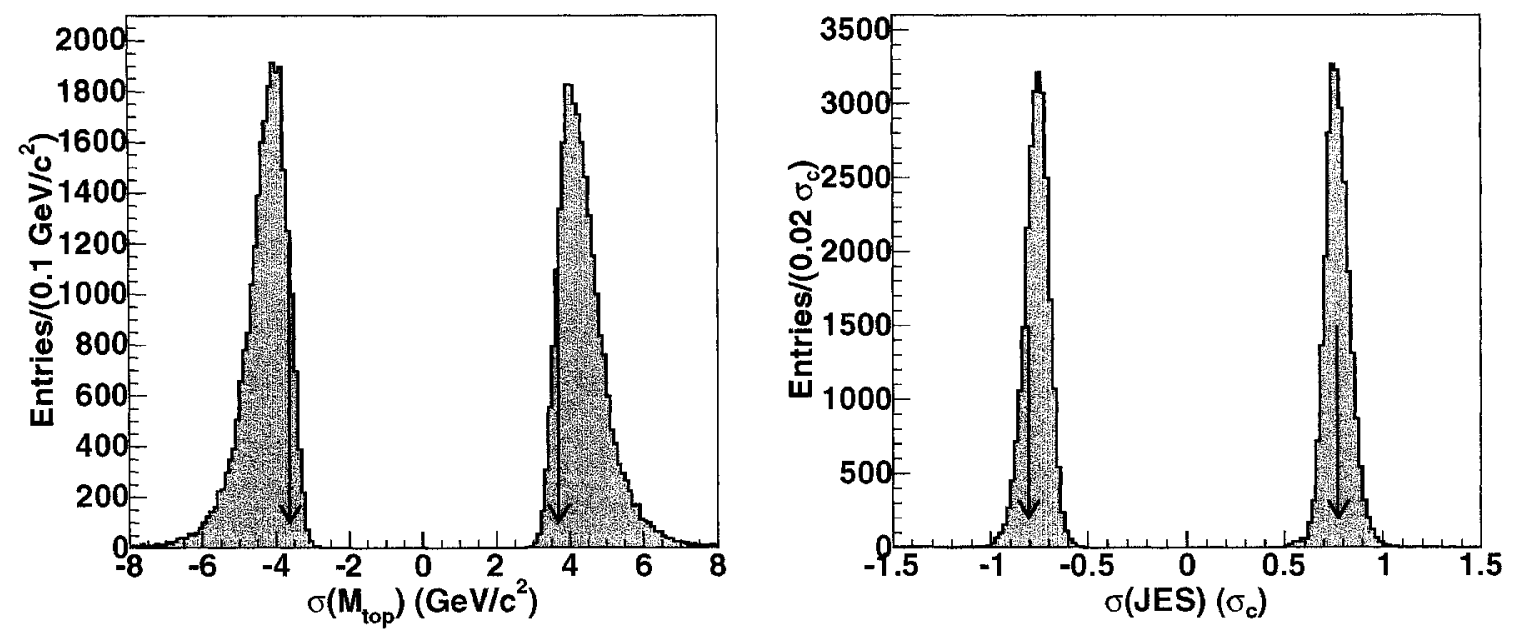

Figure 9.8: Negative and positive $M_{\text {top }}$ (left plot) and JES (right plot) uncertainties based on the number of observed events for the $M_{\text {top }}$ measurement. The arrows indicate the uncertainties obtained in data.

statistical uncertainty obtained above from the total statistical+JES uncertainty to get the JES component of the uncertainty: $\pm 2.5 \mathrm{GeV} / c^{2}$. We note that the JES uncertainty is improved by $\approx 20 \%$ with respect to the traditional technique that has a JES uncertainty of $3.1 \mathrm{GeV} / c^{2}$ [51]. This is consistent with the expected improvement based on the MC studies discussed in Sec. 7.3.3.

\subsubsection{Final Result for the $M_{t o p}$ Measurement}

We combine the statistical, JES and other systematic uncertainties to obtain the final result for the $M_{t o p}$ measurement. Adding the small background JES uncertainty of $0.04 \mathrm{GeV} / c^{2}$ (extracted in Sec. 8.7) linearly to the JES component of the uncertainty does not affect the $M_{\text {top }}$ uncertainty at a level of $0.1 \mathrm{GeV} / c^{2}$. We add the uncorrelated systematic uncertainty to obtain

$$
\left.M_{\text {top }}=173.5_{-3.6}^{+3.7} \text { (stat. }+\mathrm{JES}\right) \pm 1.3 \text { (syst.) } \mathrm{GeV} / \mathrm{c}^{2}
$$




\begin{tabular}{ccccc}
\hline \hline Subsample & Observed & Fitted $n_{s}$ & Fitted $n_{b}$ & $n_{b}$ (constr) \\
\hline 2-tag & 25 & $23.5_{-5.0}^{+5.0}$ & $1.8_{-0.5}^{+0.5}$ & $1.89 \pm 0.52$ \\
1-tag(T) & 63 & $53.9_{-7.9}^{+7.9}$ & $10.1_{-1.7}^{+1.7}$ & $10.4 \pm 1.7$ \\
1-tag(L) & 33 & $14.3_{-5.2}^{+5.2}$ & $15.5_{-2.2}^{+2.2}$ & $14.3 \pm 2.5$ \\
0 -tag & 44 & $28.3_{-8.3}^{+8.3}$ & $15.7_{-7.1}^{+8.0}$ & None \\
\hline \hline
\end{tabular}

Table 9.4: The fitted number of signal $\left(n_{s}\right)$ and background events $\left(n_{b}\right)$ in each subsample for the $M_{t o p}$ measurement. Also given are the expected number of background event that $n_{b}$ is constrained to in the likelihood fit $\left(n_{b}\right.$ (constr $\left.)\right)$.

\begin{tabular}{ccc}
\hline \hline Subsample & Fitted $n_{s}$ & Fitted $n_{b}$ \\
\hline 2-tag & $25.0_{-18.9}^{+18.9}$ & $0.0_{-0.0}^{+2.8}$ \\
1-tag(T) & $58.0_{-9.4}^{+9.4}$ & $5.0_{-0.0}^{+7.1}$ \\
1-tag(L) & $9.4_{-6.0}^{+6.0}$ & $23.6_{-6.7}^{+7.8}$ \\
0 -tag & $28.3_{-8.1}^{+8.1}$ & $15.7_{-7.1}^{+8.0}$ \\
\hline \hline
\end{tabular}

Table 9.5: The fitted number of signal $\left(n_{s}\right)$ and background events $\left(n_{b}\right)$ in each subsample for the $M_{\text {top }}$ measurement where the background is left unconstrained for all subsamples. or,

$$
M_{\text {top }}=173.5_{-2.6}^{+2.7} \text { (stat.) } \pm 2.5(\mathrm{JES}) \pm 1.3 \text { (syst.) } \mathrm{GeV} / c^{2}
$$

or equivalently,

$$
M_{\text {top }}=173.5_{-3.8}^{+3.9} \mathrm{GeV} / c^{2}
$$

For the final combined JES, we add linearly the background JES uncertainty $\left(0.033 \sigma_{c}\right)$ and add in quadrature the remaining systematic uncertainties $\left(0.33 \sigma_{c}\right)$ to obtain

$$
\mathrm{JES}=-0.10_{-0.83}^{+0.82} \text { (stat.) } \pm 0.33 \text { (syst.) } \sigma_{c},
$$

or equivalently,

$$
\mathrm{JES}=-0.10_{-0.89}^{+0.88} \sigma_{c}
$$




\begin{tabular}{ccc}
\hline \hline Subsample & $M_{\text {top }}\left(\mathrm{GeV} / c^{2}\right)$ & $\operatorname{JES}\left(\sigma_{c}\right)$ \\
\hline 2-tag & $175.0_{-4.6}^{+4.6}$ & $-0.47_{-0.84}^{+0.86}$ \\
1-tag(T) & $170.1_{-4.2}^{+4.4}$ & $0.47_{-0.88}^{+0.87}$ \\
1-tag(L) & $182.3_{-9.0}^{+10.8}$ & $0.05_{-0.96}^{+0.96}$ \\
0-tag & $153.0_{-6.6}^{+10.1}$ & $0.29_{-0.99}^{+0.98}$ \\
\hline \hline
\end{tabular}

Table 9.6: Results of the $M_{\text {top }}$ measurement in each subsample. The uncertainties are not corrected for $68 \%$ confidence level coverage. 

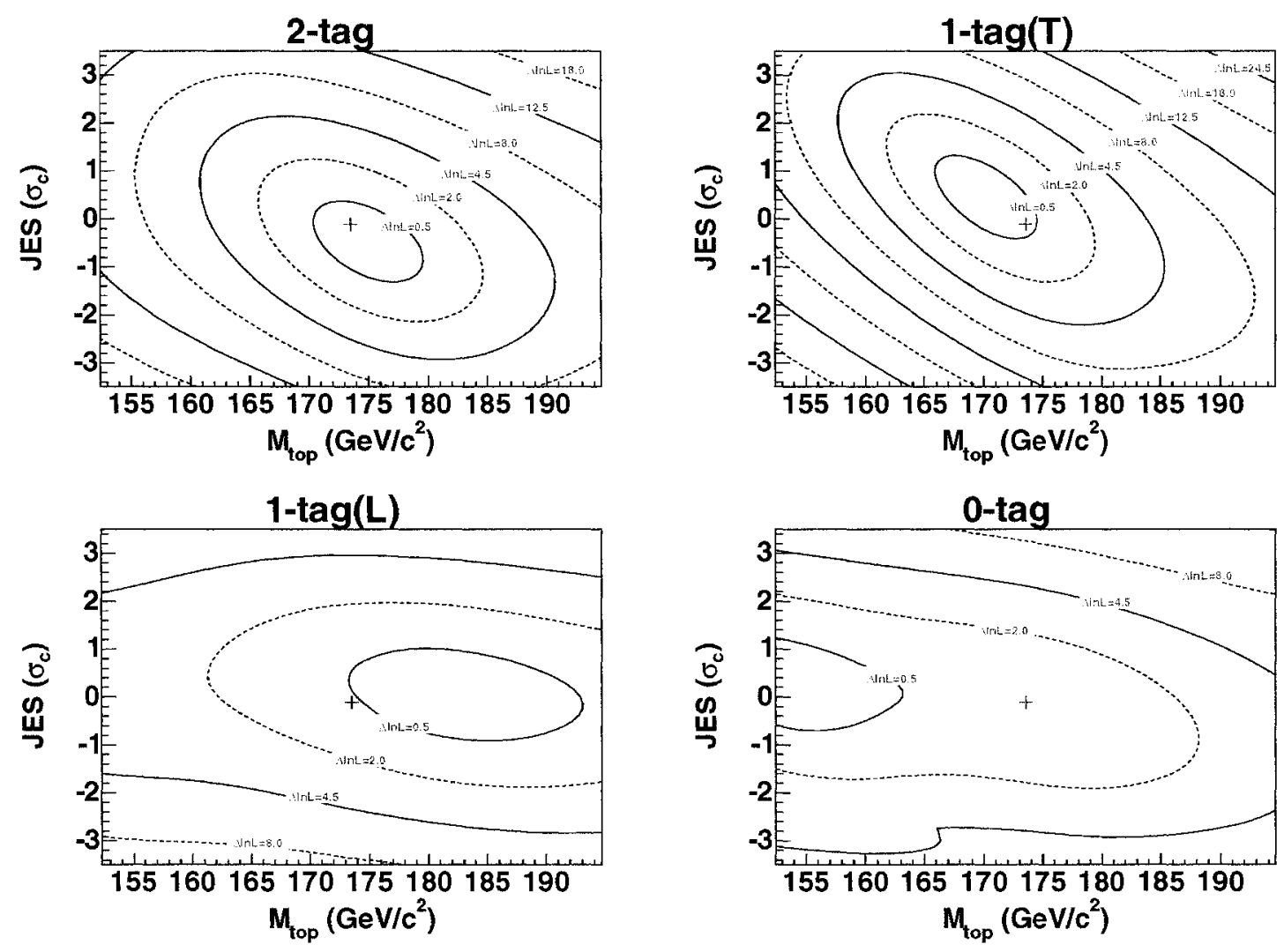

Figure 9.9: Negative log-likelihood function as a function of $M_{\text {top }}$ and JES for 2-tag (upper left), 1-tag(T) (upper right), 1-tag(L) (bottom left) and 0-tag subsamples (bottom right) for the $M_{t o p}$ measurement. The cross-hair indicates the most probable result of the combined fit. The a priori JES calibration is applied to each subsample individually. The increments in log-likelihood are chosen to represent the one-dimensional standard deviation of the parameters. (To avoid confusion, we don't label the two-dimensional curves $1 \sigma, 2 \sigma$, etc, since the equivalent coverage in the multi-dimensional space of parameters requires larger variations of the log-likelihood (see e.g. Table 31.2 of [5])). 


\section{Chapter 10}

\section{Conclusions}

We have improved the top quark mass measurement by using the largest sample of $t \bar{t}$ candidate events observed to date and by reducing the largest systematic uncertainty, the jet energy scale, by using the information associated with the hadronic $W$ boson decays $(W \rightarrow j j)$ within the $t \bar{t}$ events. The technique employed is an extension of the traditional template analysis, using templates of the reconstructed top quark and $W$ boson mass as a function of the true top quark mass and the jet energy scale. The result is obtained from an unbinned likelihood fit that considers the correlations between these two parameters and determines them simultaneously. The jet energy scale is constrained in the likelihood fit to the value determined a priori by the CDF collaboration [45].

Prior to the full top quark mass measurement, the assumption that the information on JES from $W \rightarrow j j$ decays and the a priori CDF calibration can be combined is checked by measuring JES in $t \bar{t}$ events using only the $m_{j j}$ templates (with $M_{t o p}$ fixed at $\left.178 \mathrm{GeV} / c^{2}\right)$. The fit yields

$$
\mathrm{JES}=-0.76 \pm 1.27 \sigma_{c}
$$

which is in good agreement with the CDF calibration (JES $=0 \pm 1 \sigma_{c}$ ).

The full $M_{\text {top }}$ measurement is then performed where JES is measured by both the $a$ 
priori calibration and the $W \rightarrow j j$ measurement to obtain

$$
\begin{gathered}
\left.M_{\text {top }}=173.5_{-3.6}^{+3.7} \text { (stat. }+\mathrm{JES}\right) \pm 1.3 \text { (syst.) } \mathrm{GeV} / \mathrm{c}^{2}, \\
\mathrm{JES}=-0.10_{-0.83}^{+0.82} \text { (stat.) } \pm 0.33 \text { (syst.) } \sigma_{c},
\end{gathered}
$$

or equivalently

$$
\begin{gathered}
M_{\text {top }}=173.5_{-3.8}^{+3.9} \mathrm{GeV} / c^{2}, \\
\mathrm{JES}=-0.10_{-0.89}^{+0.88} \sigma_{c} .
\end{gathered}
$$

This constitutes the best single measurement of the top quark mass to date, and by itself is more precise than the previous world average [6]. We obtain an improvement of about $10 \%$ in $M_{t o p}$ uncertainty over the traditional template method with the addition of the information from the in situ $W \rightarrow j j$ decays with the current dataset.

We emphasize that this measurement assumes that the top quarks are produced and decay in the manner predicted by the SM. Indeed, the reconstructed top quark and $W$ boson mass templates have been created from simulated SM events. The robustness of the result in the presence of physics beyond the SM has been verified for the related traditional template analysis [51] using the extreme case where every $t \bar{t}$ events is assumed to originate from a resonance with a mass of $700 \mathrm{GeV} / c^{2}$. A small average shift of $1.5 \mathrm{GeV} / c^{2}$ is observed, suggesting that template analyses are mostly insensitive to the production mode of $t \bar{t}$ events. The effect of new physics in the decay (e.g. top quark decaying to charged Higgs $t \rightarrow H^{+} b$ ) has not been explored yet, and could have a more significant effect since $m_{t}^{r e c o}$ and $m_{j j}$ are reconstructed assuming the $W$ boson daughters invariant mass corresponds to the true $W$ boson mass. However, searches for new physics in top decays have been performed and shown consistency with the SM to date [26].

We note that other top quark mass measurements could benefit from the improved JES measurement obtained from the combination of the CDF jet energy calibration and $W \rightarrow j j$ measurement (e.g. analyses in the dilepton channel that cannot use $W \rightarrow j j$ decays). In doing so, one would have to account for the correlations between JES and 
$M_{\text {top }}$ that are incorporated in this analysis by performing a simultaneous fit of these two parameters. This can be done by adding the relatively small $M_{\text {top }}$ uncertainty of $0.36 \sigma_{c}$ on the fitted JES extracted in Sec. 8.10.

A preliminary world average of $M_{\text {top }}$ has been extracted [60] using the measurement presented here and the best measurements from the CDF and D $\varnothing$ collaborations performed in the lepton+jets and dilepton channels in Run I and Run II $[10,11,12,13,14$, $61,62]:$

$$
M_{\text {top }}=172.7 \pm 1.7 \text { (stat.) } \pm 2.4 \text { (syst.) } \mathrm{GeV} / c^{2}=172.7 \pm 2.9 \mathrm{GeV} / c^{2}
$$

The new measurement is about $5 \mathrm{GeV} / c^{2}$ lower but consistent with the previous average [6]. The measurement presented here carries the higher weight in the average (36\%) followed closely by a recent measurement $\left(M_{t o p}=169.5 \pm 4.4(\text { stat. }+\mathrm{JES})_{-1.6}^{+1.7}\right.$ (syst.) $\left.\mathrm{GeV} / c^{2}\right)$ performed at $\mathrm{D} \varnothing$ in the lepton+jets channel [62] (33\%).

Updated constraints on the Higgs boson mass have been obtained [15] from the fit to all electroweak precision data (discussed in more detail in Sec. 2.2.1) and specifically to the new $M_{\text {top }}$ average, resulting in

$$
\begin{aligned}
& M_{H}=91_{-32}^{+45} \mathrm{GeV} / c^{2}, \\
& M_{H}<186 \mathrm{GeV} / c^{2} \text { at } 95 \% \text { C.L. }
\end{aligned}
$$

This can be compared with the ones obtained with the previous average given in Eqns. 2.92.10. The central value of $M_{H}$ is reduced by about $35 \mathrm{GeV} / c^{2}$ and the new $95 \%$ C.L. upper limit is reduced by about $90 \mathrm{GeV} / c^{2}$. The uncertainties on $M_{H}$ are significantly improved. The $p$-value of the fit to the SM hypothesis is similar $(\approx 15 \%)$ for both $M_{t o p}$ averages. The constraints on $M_{H}$ as a function of the measurements of $M_{t o p}$ and $M_{W}$ are shown in Fig. 10.1 for the previous (left plot) and new $M_{t o p}$ averages (right plot).

Updated constraints on Supersymmetry (discussed in more detail in Sec. 2.2.2) have been obtained using only the measurement presented here [63]. Figure 10.2 shows the allowed region for the MSSM and the SM in the $M_{t o p}-M_{W}$ plane for the Run I world 

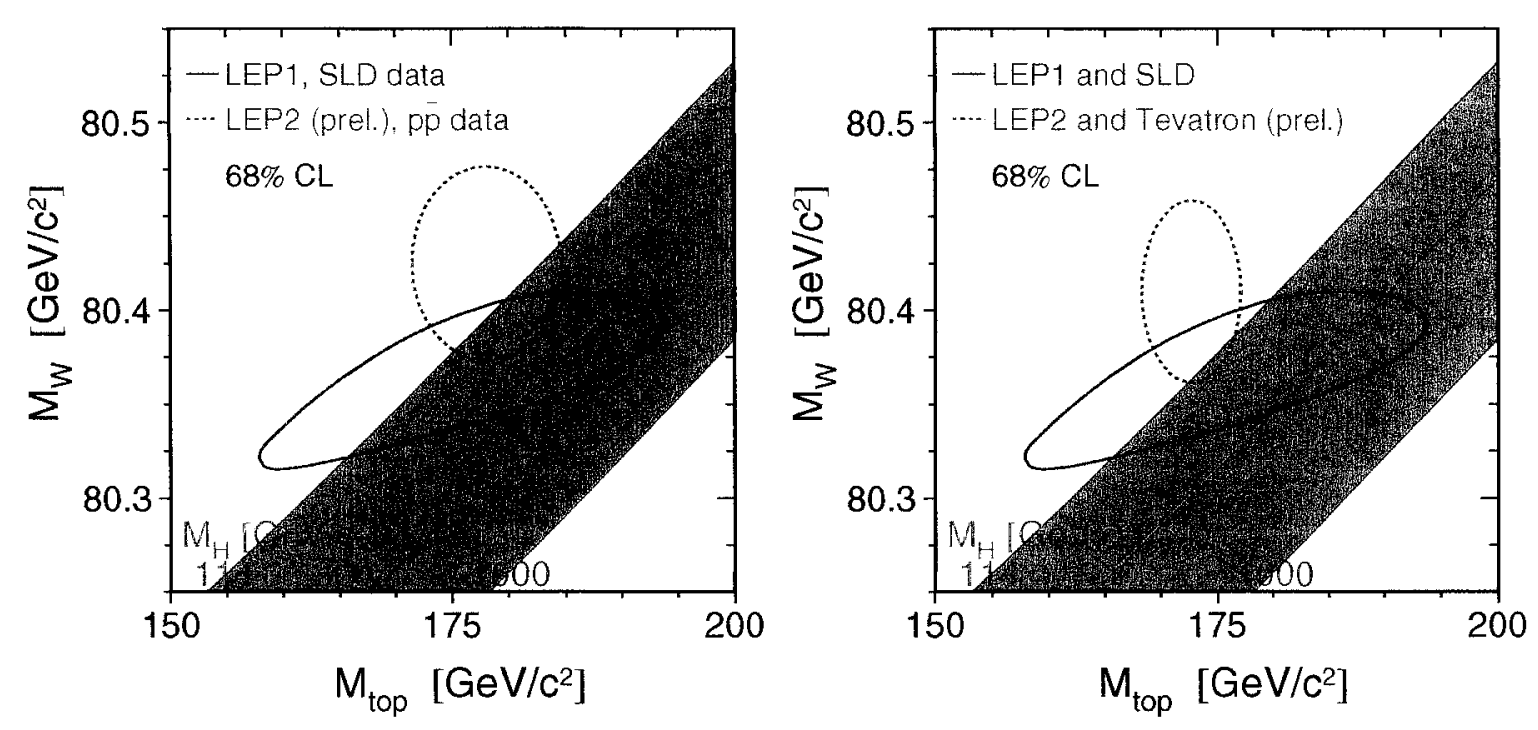

Figure 10.1: Contours of $68 \%$ confidence level in the $M_{t o p}-M_{W}$ plane and the constraint on the Higgs boson mass as a function of $M_{t o p}$ and $M_{W}$ using the previous $\left(M_{t o p}=\right.$ $\left.178.0 \pm 4.3 \mathrm{GeV} / c^{2}\right)$ and new $M_{t o p}$ world averages $\left(M_{t o p}=172.7 \pm 2.9 \mathrm{GeV} / c^{2}\right)$ on the left and right, respectively. The solid red curve shows the constraints (68\% C.L.) coming from studies at the $Z$ boson pole. The dashed blue curve shows constraints (68\% C.L.) from precise measurement of $M_{W}$ and $M_{t o p}$.

average (gray ellipse) and measurement presented here (blue ellipse). The new measurement of $M_{t o p}$ favors slightly the MSSM but better measurements of the electroweak precision observables are needed to distinguish between the two theories.

We also demonstrate the importance of the in situ determination of the jet energy scale using $W \rightarrow j j$ decays for the future of the top quark mass measurement. One significant advantage of this technique is that the JES uncertainty naturally scales with integrated luminosity, which is not necessarily the case for the traditional CDF calibration presented in Sec. 5.4. The JES uncertainty determined only from the $W \rightarrow j j$ templates is shown in Fig. 10.3 as a function of integrated luminosity. An uncertainty of $1 \mathrm{GeV} / \mathrm{c}^{2}$ or better can be achieved by the end of Run II $\left(\approx 4-8 \mathrm{fb}^{-1}\right)$, a level that will be difficult 


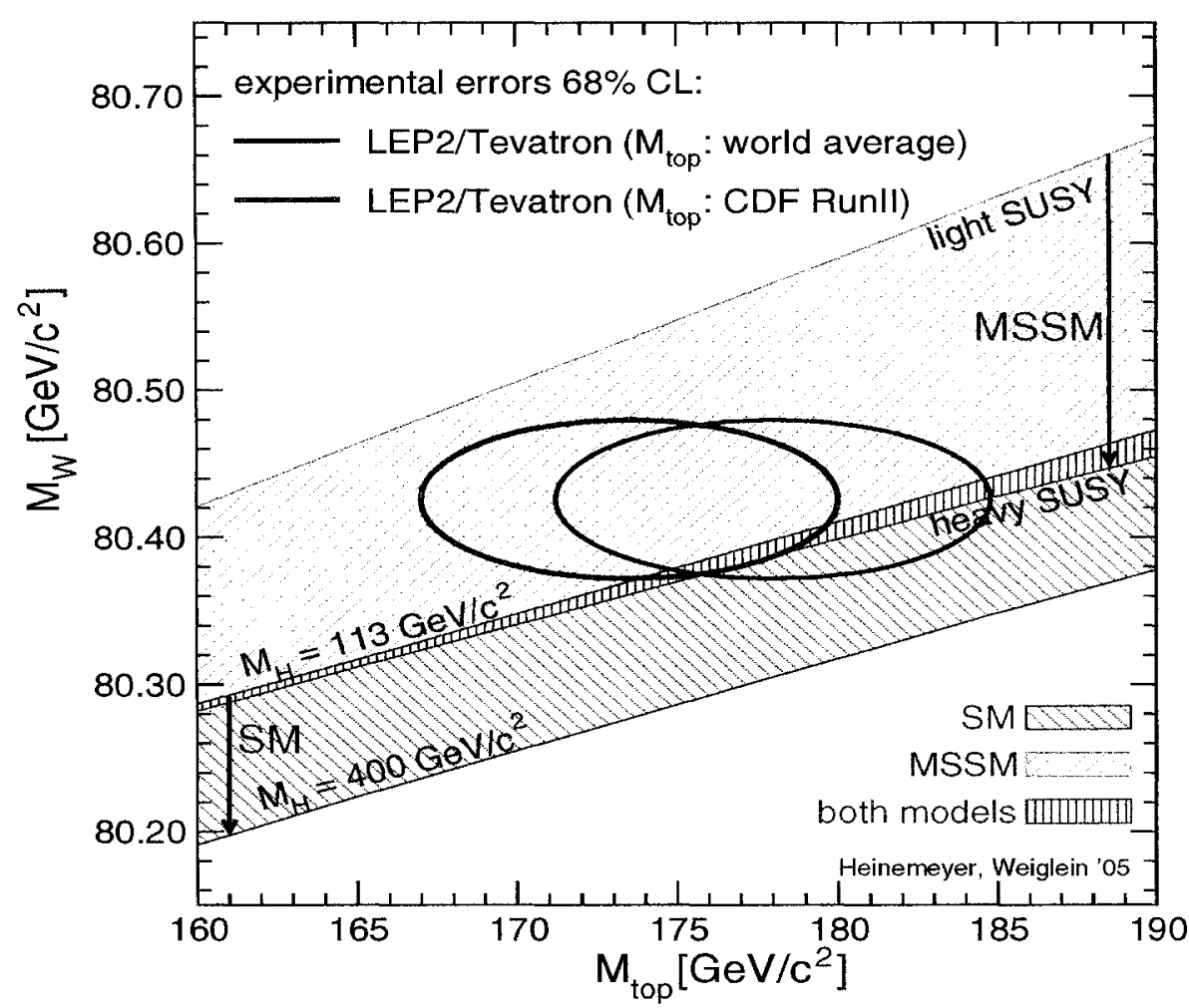

Figure 10.2: Experimental constraints on $M_{\text {top }}$ from Run I (black ellipse) and the measurement presented in this thesis (blue ellipse) and $M_{W}$. Also shown are the allowed region for MSSM (green hatched), the SM (red cross-hatched) and the overlap region between the SM and MSSM (blue vertical lines).

to reach with the traditional calibration that is intrinsically limited by our understanding of the jet fragmentation process.

The projected total $M_{\text {top }}$ uncertainty is shown in Fig. 10.4 as a function of integrated luminosity for Run II where the systematic uncertainties apart from JES are constant and equal to the current estimate $\left(1.3 \mathrm{GeV} / c^{2}\right)$. We note that this conservative estimate yields a better uncertainty than for the $M_{t o p}$ uncertainty of $3 \mathrm{GeV} / c^{2}$ for all channels projected in the CDF-II Technical Design Report [8] with $\int \mathcal{L} d t=2 \mathrm{fb}^{-1}$ (indicated on the plot). Our projection predicts that a top quark mass uncertainty of $2 \mathrm{GeV} / c^{2}$ or better can be achieved by the end of Run II $\left(\approx 4-8 \mathrm{fb}^{-1}\right)$ for a single analysis in the lepton+jets channel. This projection allows us to predict that uncertainties significantly 


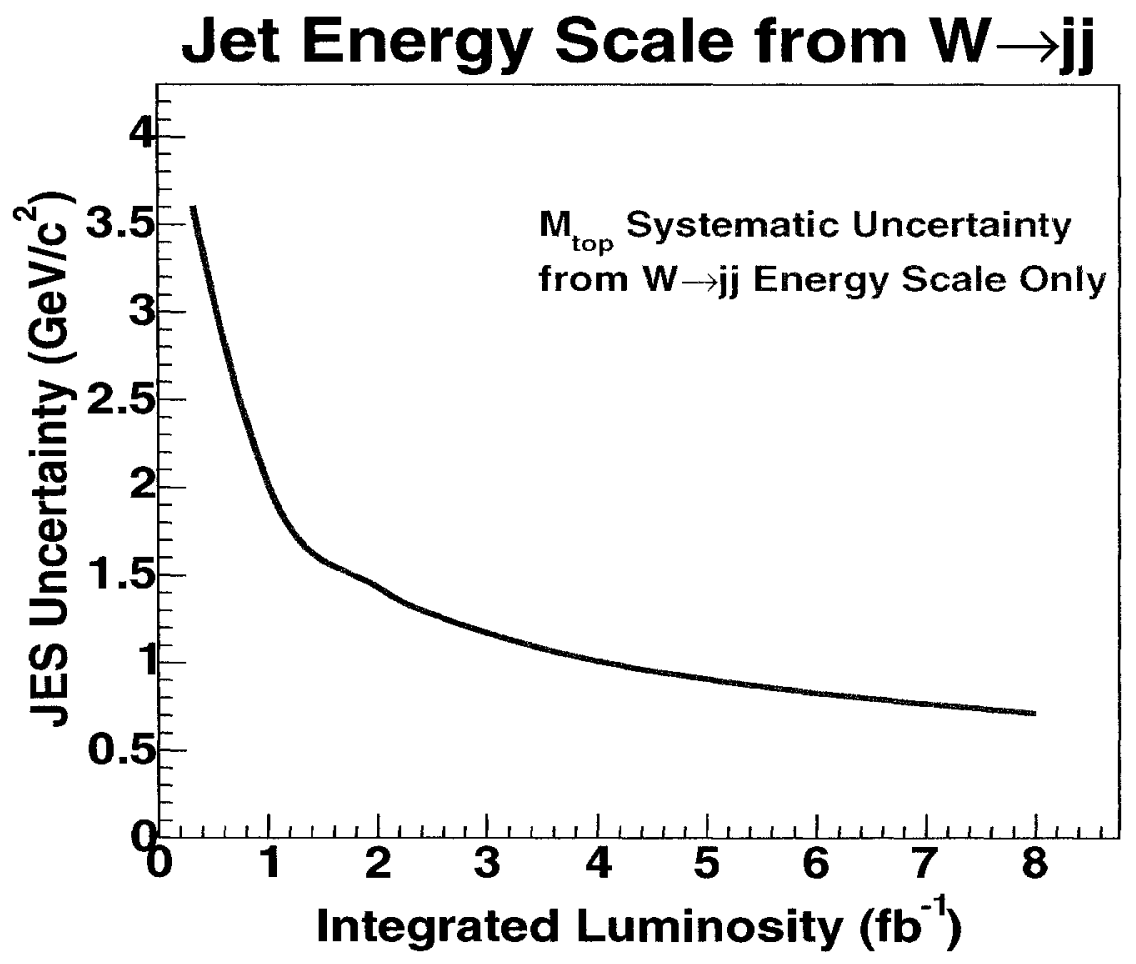

Figure 10.3: JES uncertainty from $W \rightarrow j j$ measurement only as a function of integrated luminosity.

better than $2 \mathrm{GeV} / c^{2}$ can be achieved by the end of the Run II of the Tevatron with better understanding of the systematic uncertainties, more sophisticated fitting methods and combination with other channels and the DØ experiment.

Finally we note that the current analysis provides an important "practice" for the LHC experiments where the determination of the jet energy scale is expected to rely heavily on the $W \rightarrow j j$ calibration [64] with the very large $t \bar{t}$ samples that will be available. 


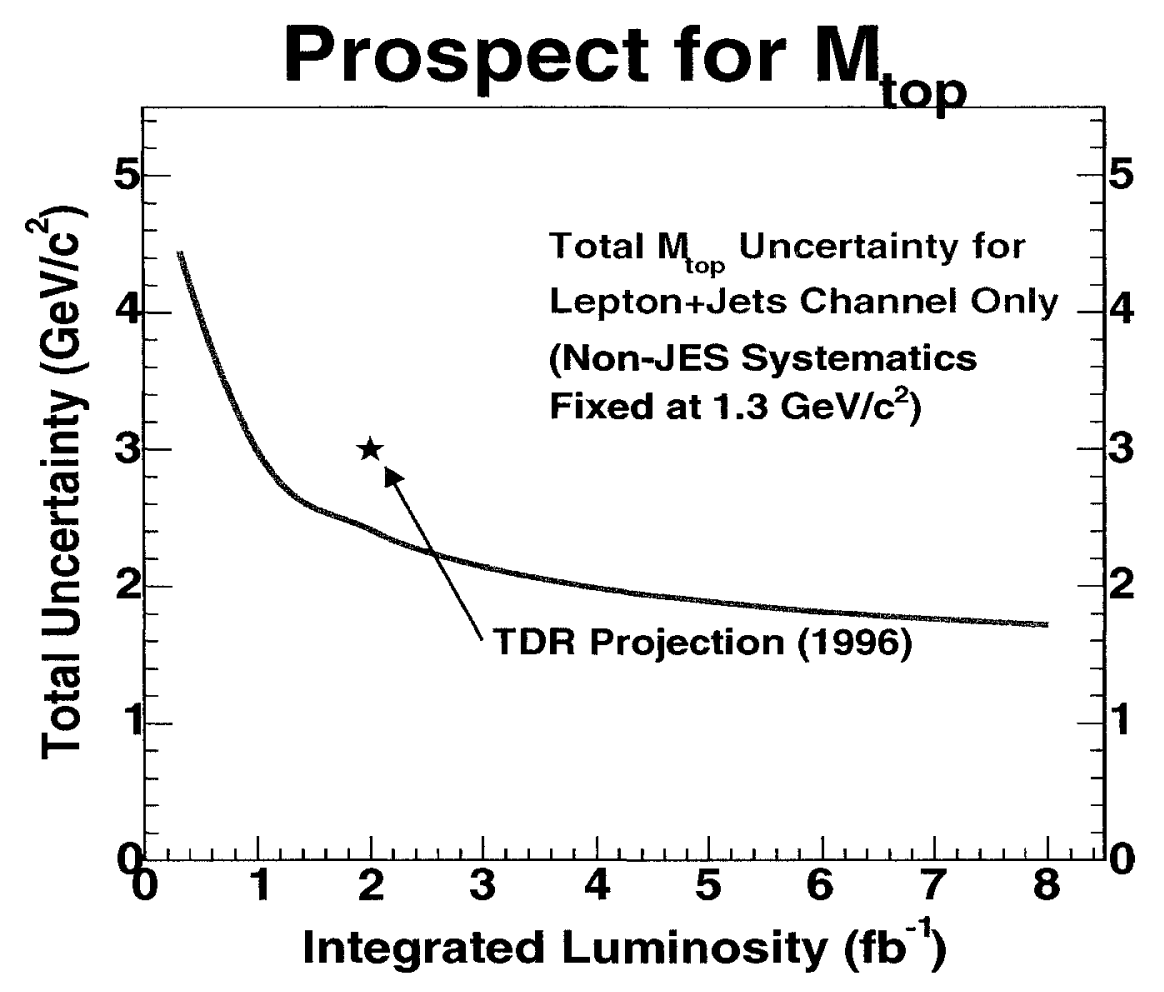

Figure 10.4: Total $M_{t o p}$ uncertainty as a function of integrated luminosity where the systematic uncertainties apart from JES are constant and equal to the current estimate. The projection of the CDF-II Technical Design Report [8] is indicated by a star. 


\section{Contributions}

My contributions to this collaborative effort include:

- Responsible of the CDF jet reconstruction software (2001-2004). Participated to the missing $E_{T}$ and calorimetry software projects.

- Development of the first underlying event and multiple interaction correction at CDF-II

- Contribution to the CDF primary vertex finder development

- Development of the top-specific jet energy corrections

- Development of the technique to reconstruct the hadronic $W$ boson invariant mass

- Development of the simultaneous two-dimensional likelihood fit of the top quark mass and the jet energy scale

- Development of the $b$-jets systematic uncertainty

- Performance of the full analysis: selection of the dataset, reconstruction of the top quark and $W$ boson mass, sanity checks, systematic uncertainties, application to data and additional checks 


\section{Glossary}

This glossary is in large part a courtesy of the Particle Data Group [65].

Accelerator: A machine used to accelerate particles to high speeds, and thus high energy compared to their rest mass energy.

Antiparticle: For most particle types (and every fermion type) there is another particle type that has exactly the same mass but the opposite value of all other charges (quantum numbers). This is called the antiparticle. For example, the antiparticle of an electron is a particle of positive electric charge called the positron. Most boson types also have antiparticles except for those that have zero value for all charges, such as a photon or a composite boson made from a quark and its corresponding antiquark. In these cases there is no distinction between the particle and the antiparticle; they are the same object. Antiquark: The antiparticle of a quark.

Baryon : A hadron made from three quarks. The proton (up-up-down) and the neutron (up-down-down) are both baryons. They may also contain additional quark-antiquark pairs.

Boson : A particle that has integer intrinsic angular momentum (spin) measured in units of $\hbar$ (spin $=0,1,2, \ldots$ ). All particles are either fermions or bosons. The particles associated with all the fundamental interactions (forces) and composite particles with even numbers of fermion constituents (quarks) are bosons.

Bottom Quark : The fifth flavor of quark (in order of increasing mass), with electric 
charge of $-1 / 3$.

Cabibbo-Kobayashi-Maskawa (CKM) Matrix : A matrix that parametrizes the rate of transition of the electroweak charge current between the various flavors of quarks. Charge : A quantum number carried by a particle. Determines whether the particle can participate in an interaction process. A particle with electric charge has electrical interactions; one with strong charge has strong interactions, etc.

Collider : A collider is an accelerator in which two beams traveling in opposite directions are steered together to provide high-energy collisions between the particles in one beam and those in the other.

Color Charge : The quantum number that determines participation in strong interactions. Quarks and gluons carry nonzero color charges.

Conservation : When a quantity is always the same before and after a particle reaction, it is said to be conserved. Such quantities include electric charge, energy, and momentum.

Decay : A process in which a particle disappears and in its place different particles appear. The sum of the masses of the produced particles is always less than the mass of the original particle.

Down Quark : The down quark (d) is the second flavor of quark (in order of increasing mass), with an electric charge $-1 / 3$.

Electric Charge : The quantum number that determines participation in electromagnetic interactions.

Electromagnetic Interaction : The interaction due to electric charge; this includes magnetic interactions

Electron : The least massive electrically-charged particle, hence absolutely stable. It is the most common lepton, with electric charge -1 .

Electroweak Interaction : In the Standard Model, electromagnetic and weak interactions are related (unified); physicists use the term electroweak to encompass both of 
them.

Event : What occurs when two particles collide or a single particle decays. Particle theories predict the probabilities of various possible events occurring when many similar collisions or decays are studied. They cannot predict the outcome for any single event. Fermion : Any particle that has odd-half-integer $(1 / 2,3 / 2, \ldots)$ intrinsic angular momentum (spin), measured in units of h-bar. All particles are either fermions or bosons. Fermions obey a rule called the Pauli Exclusion Principle, which states that no two fermions can exist in the same state at the same time. Many of the properties of ordinary matter arise because of this rule. Electrons, protons, and neutrons are all fermions, as are all the fundamental matter particles, both quarks and leptons.

Flavor : The name used for the different quarks types (up, down, strange, charm, bottom, top) and for the different lepton types (electron, muon, tau). For each charged lepton flavor there is a corresponding neutrino flavor. In other words, flavor is the quantum number that distinguishes the different quark/lepton types. Each flavor of quark and lepton has a different mass.

Generation : A set of one of each charge type of quark and lepton, grouped by mass. The first generation contains the up and down quarks, the electron and the electron neutrino. There are three generations of matter.

Gluon $(g)$ : The carrier particle of the strong interactions.

Hadron : A particle made of strongly-interacting constituents (quarks and/or gluons). These include the meson and baryons. Such particles participate in residual strong interactions.

Interaction : A process in which a particle decays or it responds to a force due to the presence of another particle (as in a collision). The four fundamental interactions are gravitational, electromagnetic, strong, and weak.

Isospin : Quantum number of a particle associated with the weak force (see below for a definition of Spin). Fermions are organised in doublets (or pairs in other words) of 
isospin in the Standard Model. For example, one such doublet is made of the electron and its associated neutrino that have respectively isospins $-1 / 2$ and $1 / 2$.

Lepton: A fundamental fermion that does not participate in strong interactions. The electrically-charged leptons are the electron $\left(e^{-}\right)$, the muon $(\mu)$, the tau $(\tau)$, and their antiparticles. Electrically-neutral leptons are called neutrinos $(\nu)$.

LHC : The Large Hadron Collider at the CERN laboratory in Geneva, Switzerland. LHC will collide protons into protons at a center-of-mass energy of about $14 \mathrm{TeV}$. When completed in the year 2007, it will be the most powerful particle accelerator in the world. It is hoped that it will unlock many of the secrets of particle physics.

Mass (or rest mass) : The rest mass (m) of a particle is the mass defined by the energy of the isolated (free) particle at rest, divided by the speed of light squared. When particle physicists use the word "mass," they always mean the "rest mass" $(m)$ of the object in question.

Meson : A hadron made from an even number of quark-antiquark constituents. The basic structure of most meson is one quark and one antiquark.

Muon $(\mu)$ : The second flavor of charged leptons (in order of increasing mass), with electric charge -1 .

Neutrino $(\nu)$ : A lepton with no electric charge. Neutrinos participate only in weak and gravitational interactions and are therefore very difficult to detect. There are three known types of neutrinos, all of which have very little mass.

Nucleus : A collection of neutrons and protons that forms the core of an atom (plural: nuclei).

Particle : A subatomic object with a definite mass and charge.

Photon $(\gamma)$ : The force carrier particle of electromagnetic interactions.

Pion $(\pi)$ : The least massive type of meson, pions can have electric charges of $+1,-1$, or 0 .

Positron $\left(e^{+}\right)$: The antiparticle of the electron. 
Proton : The most common hadron, a baryon with electric charge +1 equal and opposite to that of the electron. Protons have a basic structure of two up quarks and one down quark (bound together by gluons). The nucleus of a hydrogen atom is a proton. A nucleus with electric charge $Z$ contains $Z$ protons, which is why the number of protons is what distinguishes the different chemical elements.

Quantum : The smallest discrete amount of any quantity (plural: quanta).

Quantum Chromodynamics: The mathematical theory of the strong interaction.

Quantum Mechanics : The laws of physics that apply on very small scales. The essential feature is that electric charge, momentum, and angular momentum, as well as charges, come in discrete amounts called quanta.

Quark $(q)$ : A fundamental fermion that has strong interactions. Quarks have electric charge of either $+2 / 3$ (up, charm, top) or $-1 / 3$ (down, strange, bottom) in units where the proton charge is 1 .

Spin : A quantum particle property of intrinsic angular momentum.

Stable : Does not decay. A particle is stable if there exist no processes in which a particle disappears and in its place different particles appear.

Standard Model : Physicists have developed a theory of fundamental particles and interactions called the Standard Model.

Strange Quark $(S)$ : The third flavor of quark (in order of increasing mass), with electric charge $-1 / 3$.

Strong Interaction : The interaction responsible for binding quarks, antiquarks, and gluons to make hadrons. Residual strong interactions provide the nuclear binding force. Synchrotron : A type of circular accelerator in which the particles travel in synchronized bunches at fixed radius.

Tau $(\tau)$ : The third flavor of charged lepton (in order of increasing mass), with electric charge -1 .

Top Quark $(t)$ : The sixth flavor of quark (in order of increasing mass), with electric 
charge $2 / 3$. Its mass is much greater than any other quark or lepton.

Track : The record of the path of a particle traversing a detector.

Tracking : The reconstruction of a "track" left in a detector by the passage of a particle through the detector.

Tree Level : Particles interactions are expressed using the so-called Feynman diagrams in particle physics. These allow a visualisation of the process and are related to a systematic set of rules that are the basis of the theoretical calculations associated with the interactions in question. The calculations generally use perturbative theory, and the tree level expresses the lowest order of perturbation of the process.

Up Quark : The least massive flavor of quark, with electric charge $2 / 3$.

$W^{+}, W^{-}$Boson : A carrier particle of the weak interactions. It is involved in all electric-charge-changing weak processes.

Weak Interaction : The interaction responsible for all processes in which flavor changes, hence for the instability of heavy quarks and leptons, and particles that contain them. Weak interactions that do not change flavor (or charge) have also been observed.

Width of a particle $(\Gamma)$ : The width is related to the lifetime of an unstable particle and the Heisenberg's uncertainty principle: $\Delta E \Delta t>\hbar / 2$. The width expresses the uncertainty in measuring the particle's energy (or mass if the particle is at rest) given the particle lifetime: $\Gamma \tau=\hbar$.

$Z$ Boson : A carrier particle of the weak interactions. It is involved in all weak processes that do not change flavor. 


\section{Bibliography}

[1] J.J. Thomson, Phil. Mag. 44, 293 (1897);

J.J. Thomson, Nature 55, 453 (1897).

[2] E. Rutherford, Phil. Mag. 21, 669, (1911).

[3] H. Becquerel, Compt. Ren. 122, 762 (1896);

H. Becquerel, Compt. Ren. 122, 1086 (1896).

[4] M. Gell-Mann, Phys. Lett. 8, 214 (1964);

G. Zweig, CERN preprint CERN-8182-TH-401 (1964), unpublished;

G. Zweig, CERN preprint CERN-8419-TH-412 (1964), unpublished.

[5] S. Eidelman et al., Phys. Lett. B592, 1 (2004).

[6] P. Azzi et al., CDF and DØ Collaborations and The Tevatron Electroweak Working Group, hep-ex/0404010.

[7] G. Arnison et al., Phys. Lett. B122, 103 (1983);

G. Arnison et al., Phys. Lett. B126, 398 (1983).

[8] R. Blair et al. (CDF-II), PUB 96-390-E, FERMILAB (1996).

[9] V Abazov et al., DØ Collaboration (to be published), T. LeCompte and H.T. Diehl, Ann. Rev. Nucl. Part. Sci. 50, 71 (2000). 
[10] T. Affolder et al., CDF Collaboration, Phys. Rev. D 63, 032001 (2001);

B. Abbott et al., D $\varnothing$ Collaboration, Phys. Rev. D 58, 052001 (1998).

[11] V.M. Abazov et al., D $\emptyset$ Collaboration, Nature 429, 638 (2004).

[12] F. Abe et al., CDF Collaboration, Phys. Rev. Lett. 82, 271 (1999).

[13] B. Abbott et al., DØ Collaboration, Phys. Rev. D 60, 052001 (1999).

[14] F. Abe et al., CDF Collaboration, Phys. Rev. Lett. 79, 1992 (1997);

V.M. Abazov et al., DØ Collaboration, Phys. Lett. B606, 25 (2005).

[15] LEP Electroweak Working Group, http://lepewwg . web .cern.ch/LEPEWWG/. To be published (2005).

[16] F. Abe et al., Phys. Rev. Lett. 74, 2626 (1995);

S. Abachi et al., Phys. Rev. Lett. 74, 2632 (1995).

[17] A discussion of the motivation for a top quark inside the Standard Model can be found in: J.H. Kühn, Lectures delivered at 23rd SLAC Summer Institute, (1995); hep-ph/9707321.

[18] M. Cacciari et al. JHEP 0404, 068 (2004);

N. Kidonakis and R. Vogt, Phys. Rev. D 68, 114014 (2003).

[19] V.M. Abazov et al., DØ Collaboration, Phys. Rev. D 67, 012004 (2003).

[20] T. Affolder et al., CDF Collaboration, Phys. Rev. D 64, 032002 (2001); Erratumibid. D 67, 119901 (2003).

[21] D. Acosta et al., CDF Collaboration, Phys. Rev. D 71, 052003 (2005).

[22] D. Acosta et al., CDF Collaboration, Phys. Rev. Lett. 93, 142001 (2004). 
[23] D. Chakraborty, J. Konigsberg and D.L. Rainwater, Ann. Rev. Nucl. Part. Sci. 53, $301(2003)$.

[24] B. Abbott et al., Dø Collaboration, Phys. Rev. D Rapid Comm. 63, 031101 (2001);

V.M. Abazov et al., D $\varnothing$ Collaboration, Phys. Lett. B517, 282 (2001);

D. Acosta et al., CDF Collaboration, Phys. Rev. D 65, 091120 (2002);

D. Acosta et al., CDF Collaboration, Phys. Rev. D 68, 052003 (2004).

[25] S.L. Glashow, J. Iliopoulos and L. Maiani, Phys. Rev. D 2, 1285 (1970).

[26] F. Abe et al., CDF Collaboration, Phys. Rev. Lett. 79, 3585 (1997);

B. Abbott et al., DØ Collaboration, Phys. Rev. Lett. 82, 4975 (1999);

T. Affolder et al., CDF Collaboration, Phys. Rev. D 62, 012004 (2000);

V.M. Abazov et al., D $\varnothing$ Collaboration, Phys. Rev. Lett. 88, 151803 (2002).

[27] ALEPH Collaboration, DELPHI Collaboration, L3 Collaboration, OPAL Collaboration and The LEP Working Group for Higgs Boson Searches, Phys. Lett. B565, 61 (2003).

[28] S.P. Martin, hep-ph/9709356 (1997).

[29] H. Haber and R. Hempfling, Phys. Rev. Lett. 66, 1815 (1991);

Y. Okada, M. Yamaguchi and T. Yanagida, Prog. Theor. Phys. 85, 1 (1991);

J. Ellis, G. Ridolfi and F. Zwirner, Phys. Lett. B257, 83 (1991);

J. Ellis, G. Ridolfi and F. Zwirner, Phys. Lett. B262, 477 (1991);

R. Barbieri and M. Frigeni, Phys. Lett. B258, 395 (1991).

[30] S. Heinemeyer, W. Hollik and G Weinglein, Eur. Phys. J. C 9 (1999) 343;

G. Degrassi, S. Heinemeyer, W. Hollik, P. Slavich and G Weinglein, Eur. Phys. J. C 28 (2003) 133.

[31] S. Heinemeyer and G. Weinglein, hep-ph/0412214 (2004). 
[32] J. Erler and D.M. Pierce, Nucl. Phys. B526, 53 (1998);

G. Altarelli el al., JHEP 0106, 018 (2001);

A. Kurylov, M.J. Ramsey-Musolf and S. Nu, Nucl. Phys. B667, 321 (2003) and Phys. Rev. D 68, 035008 (2003);

W. de Boer and C. Sander, hep-ph/0307049 (2003);

S. Heinemeyer and G. Weinglein, hep-ph/0307177 (2003);

J.R. Ellis, K.A. Olive, Y. Santoso and V.C. Spanos, hep-ph/0310356 (2003).

[33] A review of dynamical electroweak symmetry breaking models can be found in:

C.T. Hill and E.H. Simmons, Phys. Rept. 381, 235 (2003); Erratum-ibid. 390, 553 $(2004)$.

[34] S. Weinberg, Phys. Rev. D 13, 974 (1976);

L. Susskind, Phys. Rev. D 20, 2619 (1979).

[35] C.T. Hill, Phys. Lett. B266, 419 (1991).

[36] E.H. Simmons, hep-ph/9908511 (1999).

[37] S. Van Der Meer et al., Phys. Rept. 58, 73 (1980).

[38] S. Klimenko, J. Konigsberg and T.M. Liss, Averaging of the Inelastic Cross Sections Measured by the CDF and the E811 Experiments, FERMILAB-FN-0741.

[39] G. Corcella, I.G. Knowles, G. Marchesini, S. Moretti, K. Odagiri, P. Richardson, M.H. Seymour and B.R. Webber, JHEP 01, 010 (2001); hep-ph/0210213 (2002).

[40] T. Sjostrand, L. Lonnblad and S. Mrenna, TP 01-21, LU (2001), hep-ph/0108264 (2001).

[41] M.L. Mangano, M. Moretti, F. Piccinini, R. Pittau and A.D. Polosa, J. High Energy Phys. JHEP 07, 001 (2003). 
[42] E. A. Gerchtein and M. Paulini, ECONF C0303241, TUMT005 (2003).

[43] R. Brun, F. Bruyant, M. Maire, A.C. McPherson and P. Zanarini (1987), CERN$\mathrm{DD} / \mathrm{EE} / 84-1$ (1984).

[44] G. Grindhammer, M. Rudowicz and S. Peters, Nucl. Instrum. Meth. A290, 469 $(1990)$.

[45] A. Bhatti et al., CDF Collaboration, Determination of the Jet Energy Scale at the Collider Detector at Fermilab, CDF Note 7543 (July 2005); To be submitted to Nucl. Instrum. Meth.

[46] D. Acosta et al., CDF Collaboration, First Measurements of Inclusive $W$ and $Z$ Cross Sections from Run II of the Tevatron Collider (to be published in Phys. Rev. D).

[47] F. Abe et al., CDF Collaboration, Phys. Rev. D 45, 1448 (1992).

[48] J.-F. Arguin and P.K. Sinervo, Revisiting the Top-Specific Jet Energy Corrections, CDF Note 6404 (April 2003).

[49] E.M. Brubaker, Ph.D. Thesis, FERMILAB-THESIS-2004-55 (2004).

[50] J. Adelman et al., Chisq optimization, matching studies and possible improvements in the Lepton+Jets top mass template, CDF Note 7533 (March 2005).

[51] D. Acosta et al., CDF Collaboration, Top Quark Mass Measurement Using the Template Method in the Lepton+Jets Channel at CDF II, CDF/ANAL/TOP/PUB/7532 (March 2005); Phys. Rev. D in preparation.

[52] F. James, MINUIT Function Minimization and Error Analysis, Reference Manual, Version 94.1, CERN Program Library Long Writeup D506 (1994). 
[53] L. Demortier and L. Lyons, Everything You Always Wanted to Know About Pulls, CDF Note 5776 (February 2002).

[54] R. Barate et al., ALEPH Collaboration, Phys. Lett. B512, 30 (2001);

K. Abe et al., SLD Collaboration, Phys. Rev. D 65, 092006 (2002);

G. Abbiendi et al., OPAL Collaboration, Eur. Phys. J. C29, 463 (2003).

[55] C. Peterson et al., Phys. Rev. D 27, 105 (1983).

[56] M.G. Bowler et al., Z. Phys. C11, 169 (1981).

[57] S. Xie and P.K. Sinervo, The Effects of Colour Flow on B Jet Energies in Top Quark Events, CDF Note 7238 (August 2004);

J.-F. Arguin and P.K. Sinervo, b-jets Energy Scale Uncertainty From Existing Experimental Constraints, CDF Note 7252 (August 2004).

[58] Y.K. Kim and U.K Yang, Initial state gluon radiation studies on Drell-Yan data for top-pair production in hadron collider, CDF Note 6804 (December 2003).

[59] G. Chlachidze et al., Validations on the data and MC for top mass analysis in Lepton+Jets channel, CDF Note 7534 (March 2005).

[60] J.-F. Arguin et al., CDF and D $\varnothing$ Collaborations and The Tevatron Electroweak Working Group, hep-ex/0507091 (2005).

[61] D. Acosta et al., CDF Collaboration, Measurement of the Top Quark Mass in the Dilepton Channel using the Leading-Order Differential Cross-Section at CDF II, CDF/ANAL/TOP/PUB/7718 (July 2005).

[62] V.M. Abazov et al., Dø Collaboration, Top Quark Measurement with the Matrix Element Method in the Lepton+Jets Final State at DØ Run II, DØ-note 4874-CONF (July 2005). 
[63] S. Heinemeyer and G. Weinglein, Private Communications (June 2005).

[64] A. Airapetian et al., ATLAS Collaboration, ATLAS detector and Physics Performance TDR Vol. 1, ATLAS TDR 14, CERN/LHCC 99-14 (May 1999);

A. Airapetian et al., ATLAS Collaboration, ATLAS detector and Physics Performance TDR Vol. 2, ATLAS TDR 15, CERN/LHCC 99-15 (May 1999).

[65] S. Eidelman et al., The Particle Data Group, The Particle Adventure, http:// particleadventure.org/particleadventure/, (2002). 